\title{
Zur Beurteilung der Sprödbruchgefährdung gelochter Stahltragwerke aus Flussstahl
}

Assessing the risk of brittle fracture of structures with holes made of old mild steel

\author{
Von der \\ Fakultät Bauingenieurwesen \\ der Technischen Universität Dresden \\ zur Erlangung der Würde eines \\ Doktor-Ingenieurs (Dr.-Ing.) \\ genehmigte
}

DISSERTATION

\author{
von \\ Dipl.-Ing. LARS SIEBER \\ geboren am 10. Oktober 1976 in Bautzen
}

Gutachter:

Prof. Dr.-Ing. Richard Stroetmann

Prof. Dr.-Ing. Michael Vormwald

Prof. Dr.-Ing. Peter Hübner

eingereicht am: 23.05.2016

verteidigt am: 26.09.2016 



\section{Vorwort}

Die Grundlagen für die vorliegende Dissertationsschrift entstanden während meiner Tätigkeit als wissenschaftlicher Mitarbeiter an der Professur für Stahlbau des Instituts für Stahl- und Holzbau der Technischen Universität Dresden. Die Arbeit enthält Ergebnisse eines mit Mitteln der Forschungsinitiative Zukunft Bau des Bundesinstitutes für Bau-, Stadtund Raumforschung (Aktenzeichen: II 3- F20-12-1-054 / SWD - 10.08.18.7-13.15) geförderten, gemeinschaftlichen Forschungsvorhabens der Technischen Universität Dresden und des Helmholtz-Zentrums Dresden-Rossendorf.

Ich danke meinem wissenschaftlichen Betreuer Herrn Prof. Dr.-Ing. R. Stroetmann für die Möglichkeit zur Promotion, den gewährten Freiraum zur Gestaltung meines Themas, seine kritische Begleitung meiner Arbeit und die Übernahme des Referats.

Dank gebührt ebenso Herrn Prof. Dr.-Ing. M. Vormwald und Herrn Prof. Dr.-Ing. P. Hübner für das in mich gesetzte Vertrauen, ihre Geduld und fachliche Unterstützung sowie die Übernahme des Koreferats.

Ebenso bedanke ich mich bei Herrn Prof. Dr.-Ing. habil. B. Zastrau für die Übernahme des Vorsitzes und Herrn Prof. Dr.-Ing. V. Mechtcherine für sein Mitwirken in der Prüfungskommission.

Ein besonderer Dank gilt Herrn Dr.-Ing. P. Langenberg für seine vielen hilfreichen Ratschläge, die für mich eine große Unterstützung bei der inhaltlichen Bewältigung und Abfassung der Arbeit waren.

Ferner danke ich meinem langjährigen Kollegen und Freund Prof. Dr.-Ing. H. Flederer für seine stete Diskussionsbereitschaft und die vielfältige Hilfe bei der Beschäftigung mit dem Thema meiner Arbeit.

Bei meinen Kolleginnen und Kollegen an der Professur für Stahlbau bedanke ich mich für ihre Unterstützung und das angenehme Arbeitsklima. Besonders danken möchte ich auch den Mitarbeitern des Helmholtz-Zentrums Dresden-Rossendorf, Herrn Dr.-Ing. H. W. Viehrig und Herrn M. Houska, wie auch den beiden Kolleginnen am Institut für Werkstoffwissenschaft, Frau Dr.-Ing. B. Vetter und Frau Dr.-Ing. V. Schubert, ohne deren engagierten Einsatz die Realisierung der umfangreichen Experimente nicht möglich gewesen wäre.

Ein herzliches Dankeschön gilt meinen Eltern, die mir meine berufliche Laufbahn ermöglicht haben sowie für ihren nicht nachlassenden Ansporn zur Fertigstellung dieser Arbeit.

Bei meiner Frau Michaela bedanke ich mich von Herzen für ihre unendliche Geduld und ihren unermüdlichen Rückhalt. Michaela, ohne Deine Unterstützung hätte ich diese Arbeit nicht beenden können. Danke für alles.

Lars Sieber 
Meiner Frau Michaela 


\section{Kurzfassung}

Bei der Beurteilung der Sicherheit bestehender Konstruktionen aus altem Baustahl (i. A. Flussstahl) und der Entscheidung über notwendige Instandsetzungs- und Verstärkungsmaßnahmen ist der Nachweis ausreichender Werkstoffzähigkeit (der Sicherheit gegen ein sprödes Versagen) von wesentlicher Bedeutung. Die in DIN EN 1993-1-10 normativ geregelten Nachweismethoden zur Beurteilung der Sprödbruchgefährdung wurden basierend auf umfangreichen bruchmechanischen Untersuchungen entwickelt. Sie gelten für Schweißkonstruktionen und „Stähle aus neuerer Zeit“ mit in der Regel hohen Zähigkeitswerten. Die Quantifizierung der Zähigkeit in Werkstoffnormen erfolgt durch Kerbschlagbiegeversuche. Die Beziehung zwischen der Übergangstemperatur $T_{27 J}$ der Kerbschlagarbeit und der Referenztemperatur $T_{0}$ der Bruchzähigkeit wird durch die modifizierte Sanz-Korrelation hergestellt, die nur für diese Stähle abgeleitet wurde.

Das in der Norm verankerte Verfahren ist für alte Flussstahlkonstruktionen mit Lochschwächung durch Niet- und Schraubenverbindungen nicht geeignet. Einerseits unterscheiden sich Kerbwirkung und Eigenspannungszustand von geschweißten und genieteten Konstruktionen und damit die Zähigkeitsanforderungen wesentlich voneinander. Auf der anderen Seite unterliegen die Zähigkeitseigenschaften von Flussstählen deutlich größeren Streuungen.

In der vorliegenden Arbeit werden experimentelle und rechnerische Untersuchungen zum Sprödbruchverhalten gelochter Konstruktionen aus altem Flussstahl vorgestellt. Wesentlicher Bestandteil sind dabei die umfangreichen Werkstoffanalysen zur Ermittlung der bruchmechanischen Werkstoffzähigkeit im spröd-duktilen Übergangsbereich nach dem Master-CurveKonzept (ASTM E1921). Die Auswertungen belegen, dass in Abhängigkeit des Herstellungsverfahrens unterschiedliche Werkstoffgüten definiert werden können. Um den Einfluss des Stanzens von Löchern auf das Sprödbruchverhalten alter Flussstähle zu beurteilen, werden Gefügeuntersuchungen und Mikrohärtemessungen durchgeführt.

Ausgehend von einer umfassenden Analyse typischer Konstruktionsformen bestehender Tragwerke des Stahlhochbaus erfolgen bruchmechanische FE-Berechnungen an Anschlüssen von Winkelprofilen zur Bestimmung der Zähigkeitsanforderungen. Die dabei gewonnenen Ergebnisse des Spannungsintensitätsfaktors werden durch Modifikation bekannter Lösungen aus der Fachliteratur für die weitere Anwendung aufbereitet.

Darauf aufbauend wird für die untersuchten Konstruktionsdetails im Rahmen einer bruchmechanischen Sicherheitsanalyse ein praxisgerechtes Verfahren zur Beurteilung der Sprödbruchgefährdung genieteter und geschraubter Bauteile abgeleitet. Mit Hilfe statistischer Methoden werden Streuungen der Festigkeits- und Zähigkeitskennwerte der Flussstähle erfasst und nach der Verifizierung durch Bauteilversuche in ein semi-probabilistisches Nachweiskonzept überführt. 


\section{Abstract}

In the safety assessment of existing structures made of old mild steel and for the decision on necessary repair and reinforcement measures, the verification of sufficient material toughness (safety against brittle failure) is essential. The normatively regulated assessment methods to avoid brittle failure in DIN EN 1993-1-10 were developed based on extensive fracture mechanics investigations. They apply to welded structures and modern steels with generally high toughness. The quantification of the material toughness in material standards is realised by Charpy impact tests. The relationship between the transition temperature $T_{27 J}$ of the notch impact energy and the reference temperature $T_{0}$ in fracture toughness is realised by the modified Sanz-correlation which has been derived for these steels.

The method is not suitable for mild steel structures with holes for riveted or bolted joints. On one hand, the stress concentrations and residual stresses of welded and riveted structures and thus toughness requirements are significantly different. On the other hand, the toughness properties of old mild steels have significantly larger variations.

In the present dissertation, experimental, analytical and numerical studies of the brittle fracture behavior of structures with holes made of old mild steel are presented. Extensive material analyses to determine the fracture toughness in the brittle-ductile transition region according to the Master Curve concept (ASTM E1921) are an essential part of this thesis. The evaluations confirm that, depending on the manufacturing process, different grades of steel can be defined. To analyse the influence of punching the holes on the brittle fracture behavior of old mild steels, structural examinations and microhardness measurements are performed.

Based on an extensive analysis of typical construction details of existing structural steelwork, fracture mechanical finite element calculations of connections of angle profiles are performed to determine their toughness requirements. The obtained results of the stress intensity factor are revised for further use by modification of known solutions from the specialised literature.

Based on the studied construction details, a practical method for assessing the hazard of brittle fracture of riveted and bolted components is derived in the framework of fracture mechanics safety analysis. Using statistical methods variations of strength and toughness properties of mild steels are considered and transferred to a semi-probabilistic assessment concept after the verification by component tests. 


\section{Inhaltsverzeichnis}

Formelzeichen und Abkürzungen $\quad$ VII

1. Einleitung und Motivation $\quad 1$

1.1. Hintergrund . . . . . . . . . . . . . . . . . . . . . 1

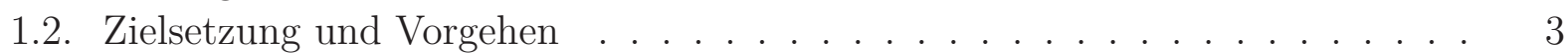

2. Stand der Wissenschaft $\quad 5$

2.1. Entwicklung der Stahlerzeugung . . . . . . . . . . . . . . . 5

2.1.1. Deutschland in den Jahren bis 1939 . . . . . . . . . . . . . . . 7

2.1.2. Das geteilte Deutschland in den Jahren 1945 bis 1949 . . . . . . . . . 8

2.1.3. Die DDR in den Jahren 1950 bis $1970 \ldots \ldots$. . . . . . . . . 8

2.2. Entwicklung der Normung für Baustähle in Deutschland . . . . . . . . . . . . 9 9

2.3. Typische Konstruktionsformen im Stahlhochbau . . . . . . . . . . . . . . . . 11

2.3.1. Allgemeines . . . . . . . . . . . . . . . . . . 11

2.3.2. Vollwandträger . . . . . . . . . . . . . . . . . . . . . . . . . . . . . 11

2.3.3. Fachwerkträger . . . . . . . . . . . . . . . . . 12

2.3.4. Dach- und Hallentragwerke . . . . . . . . . . . . . . . . . . 14

2.3.5. Stahlgeschossbauten . . . . . . . . . . . . . 15

2.3.6. Leitungsmaste und Funktürme . . . . . . . . . . . . . . . . . . 16

2.4. Das Bruchverhalten unlegierter Baustähle . . . . . . . . . . . . . 18

2.4.1. Bruchmechanismen und makroskopisches Bruchverhalten . . . . . . . 18

2.4.2. Verfestigung durch Alterung . . . . . . . . . . . . . . . . . . 19

2.4.3. Sprödbruchgefährdung gelochter Stahlkonstruktionen . . . . . . . . 20

2.5. Konzepte der Sprödbruchsicherheitsnachweise . . . . . . . . . . . . . . 22

2.5.1. Sprödbruchsicherheit nach DIN EN 1993-1-10 . . . . . . . . . . . 22

2.5.2. Bewertung der Sprödbruchneigung in Stahlgitter-Freileitungsmasten . 24

2.5.3. Sprödbruchneigung geschweißter Flussstähle . . . . . . . . . . . 26

2.6. Bruchmechanische Sicherheitsanalyse . . . . . . . . . . . . . . . . . . . . 27

2.6.1. Grundlagen der Bruchmechanik . . . . . . . . . . . . . . . . . 27

2.6.2. Spannungsintensitätsfaktoren für Lochstäbe . . . . . . . . . . . . . . . . . 32

2.6.3. Bruchmechanische Sicherheitskonzepte . . . . . . . . . . . . . . 42

2.6.4. Plastische Grenzlasten für Lochstäbe . . . . . . . . . . . . . . . . . 45

3. Experimentelle Untersuchungen an alten Baustählen $\quad 47$

3.1. Vorbemerkungen ... . . . . . . . . . . . . . . 47

3.2. Probenmaterial und Werkstoffeigenschaften . . . . . . . . . . . . 47

3.2.1. Chemische und metallografische Analyse . . . . . . . . . . . . . . . . 48

3.2.2. Festlegung der Probengeometrie und Probenherstellung . . . . . . . . 49

3.3. Festigkeitskennwerte . . . . . . . . . . . . . . . . . 50 
3.4. Werkstoffzähigkeiten . . . . . . . . . . . . . 52

3.4.1. Kerbschlagzähigkeit . . . . . . . . . . . . . . 52

3.4.2. Vergleich mit Kerbschlagzähigkeiten alter Brückenbaustähle . . . . . 55

3.4.3. Bruchmechanische Zähigkeit . . . . . . . . . . . . . . . . . . 56

3.4.4. Vergleich mit Bruchzähigkeiten alter Brückenbaustähle . . . . . . . . 62

3.4.5. Korrelationen der Werkstoffzähigkeit . . . . . . . . . . . . . . . . 64

3.5. Werkstoffeigenschaften an Loch- und Bauteilrändern . . . . . . . . . . . 69

3.5.1. Auswahl des Probenmaterials . . . . . . . . . . . . . . 69

3.5.2. Gefügeuntersuchungen . . . . . . . . . . . . . . . . 70

3.5.3. Härteverlaufsmessungen . . . . . . . . . . . . . . . . . . 72

3.6. Bauteilzugversuche . . . . . . . . . . . . . . . . . 73

3.6.1. Winkelprofilanschlüsse mit einem Verbindungsmittel . . . . . . . . 73

3.6.2. Winkelprofilanschlüsse mit mehreren Verbindungsmitteln . . . . . . . 75

4. Numerische Untersuchungen von Winkelprofilanschlüssen $\quad 77$

4.1. Grundlagen bruchmechanischer Finite-Element-Berechnungen . . . . . . . . 77

4.2. Modellierung der Winkelprofilanschlüsse . . . . . . . . . . . . . . . 78

4.3. FEM-Parameterstudie . . . . . . . . . . . . . . . . . . . 82

4.4. Gegenüberstellung mit bekannten K-Lösungen aus der Fachliteratur . . . . . 85

4.4.1. Winkelprofilanschlüsse mit einem Verbindungsmittel . . . . . . . . . 85

4.4.2. Winkelprofilanschlüsse mit mehreren Verbindungsmitteln . . . . . . . 88

5. Bruchmechanischer Sicherheitsnachweis $\quad 91$

5.1. Allgemeines . . . . . . . . . . . . . . . . . . . . . . 9 91

5.2. Statistische Auswertung nach DIN EN 1990, Anhang D . . . . . . . . . 91

5.3. Bemessungsmodelle zur Bestimmung der Grenzlast von Winkelprofilanschlüssen 93 5.3.1. Grenzlast für Winkelprofilanschlüsse mit einem Verbindungsmittel . . 93

5.3.2. Grenzlast für Winkelprofilanschlüsse mit mehreren Verbindungsmitteln 95

5.4. Bestimmung der Teilsicherheitsbeiwerte für die Bemessungsmodelle . . . . . 96

5.5. Vergleich der rechnerischen Grenzlast mit normativen Traglasten . . . . . . . 100

5.6. Zusammenfassung der Bemessungsmodelle . . . . . . . . . . . . . . . . 102

6. Anwendungsbeispiele 105

6.1. Allgemeines . . . . . . . . . . . . . . . . . . . . 105

6.2. Beispiel 1: Anschlüsse in einem Stahlgittermast . . . . . . . . . . . 105

6.3. Beispiel 2: Geschweißter Anschluss des Zuggurtes eines Fachwerkträgers . . . 107

7. Zusammenfassung und Ausblick 115

7.1. Zusammenfassung . . . . . . . . . . . . . . . . . 115

7.2. Ausblick . . . . . . . . . . . . . . . . . . 117

$\begin{array}{ll}\text { Literatur } & 119\end{array}$

A. Darstellungen der Ergebnisse aus den Werkstoffuntersuchungen $\quad 129$

B. Ergebnisse der Bauteilzugversuche 143 


\section{Formelzeichen und Abkürzungen}

In diesem Abschnitt sind häufig verwendete Symbole und Abkürzungen zusammengefasst. Dabei konnten Mehrfachdefinitionen nicht vollständig vermieden werden, da die Definitionen aus den Regelwerken und der Literatur der Bereiche Bruchmechanik und Stahlbau weitestgehend beibehalten werden sollten. Bei mehrfach verwendeten Symbolen ergibt sich die Bedeutung aus dem jeweiligen Zusammenhang.

\section{Lateinische Buchstaben}

A Koeffizient in der Approximation der Kerbschlag-Temperatur-Kurve

A Brucheinschnürung im Zugversuch nach DIN EN ISO 6892

A absolute Risslänge in den Lösungen nach Wu; CARLSSON (1991)

a Risslänge

$a_{0} \quad$ Tiefe des Initialrisses

$a_{\text {eff }} \quad$ effektive Risslänge

$a_{e q} \quad$ halbe äquivalente Risslänge

$A_{w} \quad$ Schweißnahtfläche

$B \quad$ Koeffizient in der Approximation der Kerbschlag-Temperatur-Kurve

$B \quad$ Proben- oder Bauteilbreite

$B \quad$ Lochrandabstand in der Lösung nach Forman; Shivakumar et al. (2002)

$b \quad$ Breite, z. B. Breite eines Winkelschenkels

b Mittelwertkorrektur nach DIN EN 1990, Anhang D.8

$B_{0} \quad$ Probendicke einer $\mathrm{C}(\mathrm{T})$-Probe

$b_{0} \quad$ Länge des Probenligaments einer $\mathrm{C}(\mathrm{T})$-Probe

C Koeffizient in der Approximation der Kerbschlag-Temperatur-Kurve

c halbe Rissbreite eines halbelliptischen Oberflächenrisses

c Abstand der Riss-Mittellinie zur nähergelegenen Stabkante

$C_{1}$ bis $C_{4} \quad$ Koeffizienten für Kurvenanpassungsfaktor $G_{2}$

$D \quad$ Lochdurchmesser in der Lösung nach Forman; Shivakumar et al. (2002)

d Lochdurchmesser

$\mathrm{d} s \quad$ Linienelement des Integrationsweges $\Gamma$

E Elastizitätsmodul

$e \quad$ Abstand zwischen Riss-Mittellinie und Stab-Mittellinie (Rissexzentrizität)

e Lochabstand in Kraftrichtung

$e_{1}, e_{2} \quad$ Randabstand eines Nietloches

$f \quad$ Koeffizient einer Approximationsfunktion nach LANGENBERG (1996) 
$f \quad$ Gesamt-Überlebenswahrscheinlichkeitsdichte

F Last, Kraft

$F_{a} \quad$ Rissauffangkraft

$F_{I I I} \quad$ Geometriefaktor zur Berücksichtigung der Lasteinleitung am Loch

$F_{E C C} \quad$ Geometriefaktor zur Berücksichtigung der Rissexzentrizität

$F_{F W} \quad$ Geometriefaktor zur Berücksichtigung einer endlichen Stabbreite

Frenz $\quad$ plastische Grenzlast nach Kumar; German et al. (1981)

$F_{g y} \quad$ plastische Grenzlast (generell yield)

$F_{H P W} \quad$ Geometriefaktor zur Berücksichtigung einer endlichen Stabbreite und -höhe

$F_{L} \quad$ Geometriefaktor zur Berücksichtigung eines Winkelprofilquerschnitts

$f_{n} \quad$ Spannungsintensitätsfaktor in den Lösungen nach Wu; CARLSson (1991)

$F_{P} \quad$ Geometriefaktor zur Berücksichtigung der Lasteinleitung am Loch

$f_{T} \quad$ Wahrscheinlichkeitsdichte

$f_{T_{0}} \quad$ Überlebenswahrscheinlichkeitsdichte

$F_{T C H} \quad$ Geometriefaktor zur Berücksichtigung eines Durchrisses am Loch nach Forman; SHIVAKUmar et al. (2002)

$F_{T H} \quad$ Geometriefaktor zur Berücksichtigung eines Durchrisses am Loch nach BALL (1987)

$f_{u} \quad$ Zugfestigkeit

$f_{y} \quad$ Streckgrenze

$F_{Y} \quad$ Fließlast

$G_{0}, G_{1}$, Koeffizienten des Geometriefaktors $F_{T C H}$ nach Forman; Shivakumar et al. $G_{w} \quad(2002)$

$g_{r t} \quad$ Widerstandsfunktion, die das Bemessungsmodell beschreibt

$g$

$h$

$h_{1}$

$h_{\text {eff }}$

$J$

Abstand zwischen Riss-Mittellinie und Wirkungslinie der Einzellast

Randabstand des Risses von der Bauteilkante in Kraftrichtung

Koeffizient des J-Integral-Anteils $J_{p l}$ nach Kumar; German et al. (1981)

J-Integral

$J_{e l} \quad$ elastischer Anteil des J-Integrals

$J_{p l} \quad$ plastischer Anteil des J-Integrals

$k$

Koeffizient einer Approximationsfunktion nach LANGENBERG (1996)

$K_{0} \quad$ Skalenparameter der Master-Curve

$K_{I} \quad$ Spannungsintensitätsfaktor (Mode I)

$K_{I c} \quad$ kritischer Wert des $K$-Faktors, Bruchzähigkeit

$K_{I, e f f} \quad$ Spannungsintensitätsfaktor bei Ansatz von $a_{e f f}$ (Kleinbereichsfließen)

$K_{I, F E M} \quad$ Spannungsintensitätsfaktor berechnet mittels FEM

$K_{I}^{p} \quad$ Spannungsintensitätsfaktor infolge primärer (äußerer) Beanspruchung

$K_{I}^{s} \quad$ Spannungsintensitätsfaktor infolge sekundärer Beanspruchung

(Eigenspannungen)

$K_{I p}^{s} \quad$ plastizitätskorrigierter K-Faktor 
$k_{c} \quad$ Korrekturfaktor nach DIN EN 1990

$k_{d, n}, k_{d, \infty} \quad$ Fraktilenfaktor für Bemessungswerte nach DIN EN 1990, Tabelle D.2

$K_{J}$ äquivalenter Spannungsintensitätsfaktor ermittelt aus dem J-Integral

$K_{J c}$ Bruchzähigkeit ermittelt aus dem J-Integral $J_{c}$

$K_{J c-1 T}$ Bezugswert der Bruchzähigkeit einer 1T-C(T)-Probe

$K_{J c-1 T, 5 \%}$ $5 \%$-Fraktilwert der Bruchzähigkeit

$K_{J c-1 T, 5 \%}^{M M}$ $5 \%$-Fraktilwert der Bruchzähigkeit nach der multimodalen MC-Auswertung

$K_{J c(\text { limit })}$ Messkapazität einer C(T)-Probe nach ASTM E 1921

$K_{J c(\text { med })} \quad$ Median-Bruchzähigkeit

$k_{m}$ Lagenfaktor mehrlagiger Schweißnähte

$K_{\text {mat }}$

$K_{\min }$ Bruchzähigkeit / Risswiderstand

$k_{n}, k_{\infty}$ Parameter der Master-Curve

$K_{P}$

$K_{R}$

$K_{r}$

$K V_{2}$

$L_{r}$

$L_{r, \max }$

$m$

$M_{\text {limit }}$

$N$

$n$

$n$

$N_{\text {exp }}$

Fraktilenfaktor für charakteristische Werte nach DIN EN 1990, Tabelle D.1

西 Spannungsintensitätsfaktor infolge Einleitung einer Einzellast am Loch Spannungsintensitätsfaktor infolge Zugspannung im Bruttoquerschnitt Ordinate des FAD, Verhältniswert der Rissspitzenbeanspruchung zur Bruchzähigkeit

$N_{G r, d}$

$N_{\text {grenz }}$ Kerbschlagarbeit

Abszisse des FAD, Plastizierungsgrad des Ligaments

Grenzwert der Ligamentplastizierung

Koeffizient einer Approximationsfunktion nach LANGENBERG (1996)

$N_{G r, k}$

$N_{R, d}$

$n_{\text {Riss }}$

$N_{u, R d}$

$P$ Faktor für das Deformationskriterium der C(T)-Probe nach ASTM E 1921 Exponent der Ramberg-Osgood-Beziehung Koeffizient einer Approximationsfunktion nach LANGENBERG (1996) Anzahl

experimentell ermittelte Bruchlast

Bemessungswert der Grenzlast

Grenztragfähigkeit eines Winkelprofilanschlusses

charakteristischer Wert der Grenzlast

Normative Traglast

Anzahl der Nietlöcher, die in der Risslinie liegen

$P$

Bemessungswert der Traglast nach DIN EN 1993-1-8

Einzellast

$P_{B P}$

$P_{F}$

$P_{f}$

Koeffizient des Geometriefaktors nach NEWMAN (1971)

Zugkraft im Bauteil beiderseits des Lochs

Einzellast des Verbindungsmittels am Lochrand

$P_{\text {total }}$

Versagenswahrscheinlichkeit

$Q, Q_{\delta}, Q_{r t}$

Gesamtzugkraft im Bauteil

Hilfswerte zur Bestimmung des charakteristischen Werts der Widerstandsfunktion nach DIN EN 1990, Anhang D 


\begin{tabular}{ll}
$R$ & Radius \\
$r$ & Anzahl der Proben für die Master-Curve-Auswertung \\
$r_{d}$ & Bemessungswert der Widerstandsfunktion \\
$R_{e}$ & Streckgrenze \\
$r_{e}$ & Mittelwert der experimentellen Werte des Widerstandes \\
$R_{e L}$ & untere Streckgrenze \\
$R_{e L, 5 \%}$ & $5 \%$-Fraktilwert der unteren Streckgrenze \\
$R_{e L, m}$ & Mittelwert der unteren Streckgrenze \\
$r_{k}$ & charakteristischer Wert der Widerstandsfunktion \\
$R_{m}$ & Zugfestigkeit \\
$r_{p l}$ & Ausdehnung der plastischen Zone im Ligament \\
$r_{t}$ & theoretische Widerstandsfunktion \\
$T$ & Temperatur, Prüftemperatur \\
$T_{0}$ & Referenztemperatur der Master-Curve \\
$T_{0, M M}$ & Referenztemperatur der multimodalen Master-Curve-Auswertung \\
$T_{27 J}$ & Übergangstemperatur der Kerbschlagarbeit \\
$T_{100}$ & Temperatur, bei der die Bruchzähigkeit $K_{I c}=100$ MPa $\sqrt{\mathrm{m}}$ beträgt \\
$t$ & Blechdicke, Winkelschenkeldicke \\
$U$ & potentielle Energie \\
$u$ & Verschiebung \\
$\vec{u}$ & Verschiebungsvektor entlang des Integrationsweges $\Gamma$ \\
$V$ & Variationskoeffizient \\
$v$ & Verschiebung \\
$V_{\delta}, V_{r}, V_{r t}$ & Variationskoeffizienten nach DIN EN 1990, Anhang D \\
$W$ & Stabbreite \\
$w, w_{1}, w_{2}$ & Wurzelmaß eines Nietloches im Winkelprofil \\
$w_{1}, w_{2}$ & Nahtbreite einer Schweißnaht \\
$W_{e}$ & Formänderungsarbeit \\
$W_{e f f}$ & effektive Stabbreite \\
$W_{L}$ & Länge der Mittellinie eines Winkelprofils \\
$x$ & Abstand in Ausbreitungsrichtung des Risses \\
$\underline{X}, \underline{Y}$ & Zufallsgrößen \\
$\underline{x}_{i} \underline{y}_{i}$ & i-te Realisierung der Zufallsgröße $\underline{X}$ bzw. $\underline{Y}$ \\
$Y_{C A L C}$ & bezogener Spannungsintensitätsfaktor auf Basis einer Literaturlösung \\
$Y_{F E M}$ & bezogener Spannungsintensitätsfaktor auf Basis einer FEM-Berechnung \\
$z$ & Koeffizient einer Geometriefunktion nach FoRMAN; SHIVAKUMAR et al. \\
& (2002) \\
& \\
\hline
\end{tabular}

\section{Griechische Buchstaben}

$\alpha \quad$ Koeffizient der Ramberg-Osgood-Beziehung 


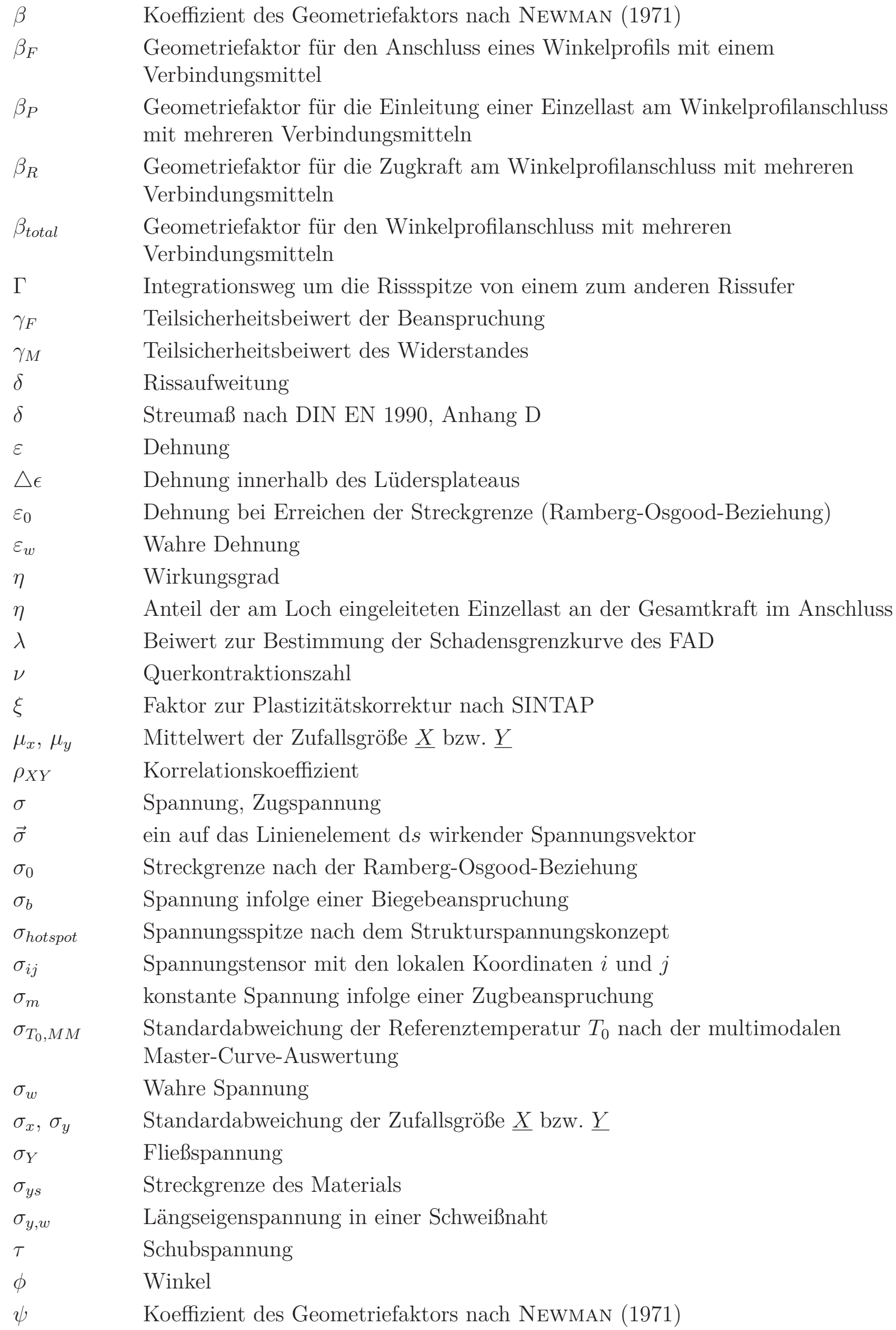




\section{Abkürzungen}

ASTM American Society for Testing and Materials

BAM Bundesanstalt für Materialprüfung

BNetzA Bundesnetzagentur

CCT Center Cracked Tension

CDF Crack Driving Force

C(T)-Probe Compact Tension (Bruchmechanikprobe)

CTOD Crack Tip Opening Displacement

DASt Deutscher Ausschuß für Stahlbau

DB Deutsche Bahn

DECT Double Edge Cracked Tension

DIN Deutsches Institut für Normung

EDZ ebener Dehnungszustand

EPBM elastisch-plastische Bruchmechanik

EPRI Electric Power Research Institute

ESZ ebener Spannungszustand

FAD Failure Assessment Diagramm

FEM Finite Elemente Methode

FKM Forschungskuratorium Maschinenbau

IEHK Institut für Eisenhüttenkunde

JCSS Joint Committee on Structural Safety

LEBM linear-elastische Bruchmechanik

MC-Methode Master-Curve-Methode

RT Raumtemperatur

SECT Single Edge Cracked Tension

SINTAP Structural Integrity Assessment Procedures for European Industry

FITNET European Fitness-for-service Network

VDE Verband der Elektrotechnik, Elektronik, Informationstechnik e. V.

V.S.F. Vorschrift für den Bau von Starkstrom-Freileitungen 


\section{Kapitel 1.}

\section{Einleitung und Motivation}

\subsection{Hintergrund}

Eine immer größer werdende Aufgabe im Bauwesen stellen die Sanierung und Ertüchtigung bestehender Bauwerke dar. Nach SARJA (2002) beträgt der wirtschaftliche Wert der bestehenden Baukonstruktionen in Europa ca. 70 bis $80 \%$ des Nationalvermögens. Dieser Baubestand ist aus wirtschaftlichen aber nicht zuletzt auch aus denkmalpflegerischen Gründen erhaltenswert. Der Stahlhochbau bildet dabei einen wichtigen Teil des Gebäudebestandes, insbesondere mit den Hallen- und Geschossbauten des 20. Jahrhunderts. In diesem Zusammenhang wurden von UnGERMANN (2008) die Konstruktionen zahlreicher erhaltener Stahlhallentragwerke der Industriearchitektur des Ruhrgebietes untersucht. Recherchen von TASche (2015) belegen zahlreiche erhaltenswerte Stahlhochbauwerke, wie Flugzeug-, Messe- oder Industriehallen. Diese Industriedenkmale sind Ausdruck gesellschaftlicher Leistung sowie Zeugnis baulicher Entwicklung. Der Wille, diese zu bewahren, unterstützt den Trend zur Umnutzung dieser Bauwerke zu Kulturbauten (Kulturkraftwerk Dresden), Veranstaltungshallen (Postbahnhof Berlin), Museen (Dieselkraftwerk Cottbus), Hotels (Erlweinspeicher Dresden) und Universitätsgebäuden (Campus Kammgarn der FH Kaiserslautern). Aber auch viele bestehende Kulturbauten (Schauspielhaus Dresden), Kirchen (Kreuzkirche Dresden) usw. besitzen stählerne Dachtragwerke, die es im Rahmen einer sachgerechten Sanierung zu erhalten gilt.

Zahlreiche dieser erhaltenen Stahltragwerke des 19. und frühen 20. Jahrhunderts werden also heute noch genutzt und haben somit ihre geplante Nutzungsdauer von 60 bis 80 Jahren bereits deutlich überschritten. Die Bahnhöfe in Deutschland weisen ein Durchschnittsalter von über 100 Jahren auf. Im Hinblick auf eine Sanierung und teilweise Umnutzung dieser Gebäude zu modernen Multifunktionsbauwerken, die Reisen, Einkaufen, Gastronomie und Kultur vereinen, werden auch in Zukunft beträchtliche Instandsetzungs- oder Umbaumaßnahmen notwendig sein [STEGUWEIT (1998)]. Aber auch die zahlreichen Bahnbetriebsanlagen stellen einen erheblichen Bauwerksbestand dar. So befinden sich allein im Regionalbereich Südost der DB Station \& Service AG über 300 Stahlhochbauwerke (Bahnhofshallen, Bahnsteigüberdachungen, Wartungshallen, etc.) aus den Baujahren vor 1950 [Deutsche BAhn (2012)].

Einen ähnlich großen Bestand an alten Stahlkonstruktionen stellen die zahlreichen Stahlgittermaste im Energieversorgungsnetz Deutschlands dar. Allein im Versorgungsnetz des Netzbetreibers RWE standen gemäß Roos (2005) ca. 28.000 Freileitungsmaste seit den 1920er Jahren in Betrieb. Sie wurden nachweislich aus im Thomasverfahren hergestelltem Flussstahl gefertigt. 
Bedingt durch Schadensfälle infolge von Sprödbruchversagen bei Altstahlkonstruktionen (z. B. an Stahlgeschossbauten und Stahlgittermasten) gewinnt neben der Tragsicherheit die Frage der Sprödbruchgefährdung alter Stahlkonstruktionen zunehmend an Bedeutung. Alte Stahlkonstruktionen bestehen überwiegend aus genieteten oder geschraubten Flachstählen, I-, L- und U-Profilen mit wiederkehrenden Konstruktionsprinzipien. Besonders sprödbruchgefährdet sind Bereiche in höher zugbeanspruchten Bauteilen aus stickstoffreichen Stählen, die durch gestanzte Löcher geschwächt sind.

Zur Beurteilung der Sprödbruchsicherheit stehen im Stahlbau verschiedene, unterschiedlich aufwendige und aussagefähige Methoden zur Verfügung, die in mehr oder weniger engem Zusammenhang mit dem eigentlichen Phänomen des Sprödbruchs stehen. Für die Wahl der Stahlgütegruppe von Schweißkonstruktionen wurde 1973 von Klöppel und Bierett die DASt-Ri. 009:1973 erarbeitet, bei der für die Sprödbruchgefährdung relevante Kriterien, wie Blechdicke, Einsatztemperatur und Eigenspannungszustand herangezogen werden. Die Beurteilung der Stähle in Bezug auf ihre Neigung zum Sprödbruchversagen erfolgt in dieser Richtlinie nach der Übergangstemperatur $T_{27 J}$ der Kerbschlagarbeit. Dieses Kriterium hat sich über die Jahrzehnte vor allem deswegen durchgesetzt, weil der Kerbschlagbiegeversuch im Unterschied zu anderen, meist komplizierteren Prüfmethoden (z. B. dem bruchmechanischen Versuch), einen deutlich geringeren Aufwand erfordert. Die Vorgehensweise zur Ermittlung der Zähigkeitsanforderungen beruhte in der DASt-Richtlinie jedoch ausschließlich auf empirischen Kriterien (Vergleich von Bauteilversuchsergebnissen mit Zähigkeiten der Stähle der Versuchskörper und Berücksichtigung verschiedener konstruktiver Details).

Die Weiterentwicklung der DASt-Richtlinie 009 erfolgte auf der Grundlage der Bruchmechanik im Zuge der Harmonisierung europäischer Normen im Eurocode 3 (DIN EN 1993-110:2010). Der Nachweis erfolgt in Form eines Temperaturvergleiches zwischen der Einsatztemperatur und dem Mindestwert der Kerbschlagübergangstemperatur $T_{27 J}$. Auf der Einwirkungsseite wird also neben der Beanspruchung des Bauteils dessen tiefste Einsatztemperatur berücksichtigt. Unter Annahme eines rissartigen Fehlers im Bauteil wird unter der maßgebenden Beanspruchung die erforderliche Bruchzähigkeit ermittelt. Die Widerstandsseite des bruchmechanischen Sprödbruchnachweises bildet die Bruchzähigkeit von Baustählen auf Basis des Master-Curve-Konzepts, anhand der aus Einsatztemperatur und Mindestbruchzähigkeit die erforderliche Referenztemperatur $T_{0}$ der Bruchzähigkeit bestimmt wird. Durch die Korrelation der Übergangstemperaturen $T_{0}$ und $T_{27 \mathrm{~J}}$ kann der Mindestwert der Kerbschlagübergangstemperatur abgeleitet werden. Dadurch wird bei der Anwendung des Sprödbruchnachweises die aufwendige experimentelle Ermittlung der Bruchzähigkeit vermieden und das bisher bekannte $T_{27 J}$-Kriterium beibehalten.

Diese Korrelation wurde speziell für heutige Baustähle in Schweißkonstruktionen abgeleitet. Die besonderen Eigenschaften alter Stähle fanden dabei keine Berücksichtigung. Auch die häufig geringere Kerbwirkung in alten genieteten Konstruktionen, die auf der Einwirkungsseite einfließt, wird im aktuellen Sprödbruchkonzept nicht berücksichtigt. Dennoch ist in der Praxis zu beobachten, dass die Grenzwerte der Kerbschlagübergangstemperatur aktueller Regelwerke auch bei der Beurteilung alter Stahlkonstruktionen angewendet werden. Dies kann aus vorgenannten Gründen zu einer Fehleinschätzung der Zähigkeitsanforderungen führen und vielfach unnötige Verstärkungsmaßnahmen oder sogar den vorsorglichen Rückbau der Konstruktion verursachen.

In bereits durchgeführten Werkstoffuntersuchungen, z. B. von HENSEN (1992) oder LANGENBERG (1996), wurde festgestellt, dass Flussstähle auch bei tiefen Temperaturen (in Deutsch- 
land für Außenbauteile bis $-30^{\circ} \mathrm{C}$ ) durchaus noch ausreichende Zähigkeit besitzen, um einem spröden Bauteilversagen zu widerstehen.

Da keine ausreichend abgesicherten Grundlagen zur Bewertung der werkstoff- und konstruktionsbedingten Sprödbruchgefährdung alter Baustähle vorliegen, ist es erforderlich, Methoden für eine sachgerechte Beurteilung zu entwickeln. In diesem Zusammenhang sind sowohl Untersuchungen auf der Einwirkungsseite als auch auf der Seite des Werkstoffwiderstandes durchzuführen.

\subsection{Zielsetzung und Vorgehen}

Die Bewertung der Sprödbruchgefährdung von Stahlbauteilen in bestehenden Konstruktionen, auf Basis der Bruchmechanik, ist in der Praxis nur wenig verbreitet und war bisher nur auf ausgewählte Bauwerke, insbesondere zyklisch beanspruchte Brücken, beschränkt (z. B. bei Sedlacek; Hensen et al. (1992) oder Brüning; Richter et al. (2005)). Dies ist neben den zum Teil notwendigen, komplexen FEM-Berechnungen vor allem auf die aufwendige, experimentelle Bestimmung der bruchmechanischen Werkstoffzähigkeit zurückzuführen.

Ein ingenieurmäßig anwendbares Nachweisverfahren, wie es mit DIN EN 1993-1-10:2010 für neue geschweißte Konstruktionen vorliegt, steht für bestehende Stahlkonstruktionen bisher nicht zur Verfügung. Die Zielstellung dieser Arbeit ist die Entwicklung eines praxisgerechten Verfahrens zur Beurteilung der Sprödbruchgefährdung lochgeschwächter Konstruktionen aus alten Baustählen. Grundlagen hierfür bilden experimentell bestimmte Werkstoffkennwerte, Finite Element Berechnungen zur Bestimmung der Zähigkeitsanforderungen typischer Konstruktionsformen und bruchmechanische Sicherheitsanalysen. Mit Hilfe statistischer Methoden werden Streuungen der Festigkeits- und Zähigkeitskennwerte der Flussstähle erfasst und nach der Verifizierung durch Bauteilversuche in ein semi-probabilistisches Nachweiskonzept überführt.

In der vorliegenden Dissertation werden mehrfach Text, Tabellen und Abbildungen aus dem Abschlussbericht des Forschungsvorhabens „Beurteilung der Sprödbruchgefährdung gelochter Stahltragwerke - Weiterentwicklung der Analysemethoden" (Stroetmann; SieBer et al. (2015)) übernommen. Auf die vollständige Angabe aller experimentellen Daten wird in dieser Arbeit verzichtet. Diese können dem Abschlussbericht entnommen werden. Dissertation und Abschlussbericht wurden vom selben Autor verfasst. Deshalb wird im Text in den entsprechenden Passagen auf die Zitatangabe verzichtet.

Zur Quantifizierung der Bruchzähigkeit von Flussstählen werden im spröd-duktilen Übergangsbereich bruchmechanische Werkstoffzähigkeiten bestimmt und nach dem international anerkannten Prüfstandard ASTM E 1921 (Master-Curve-Konzept) ausgewertet. Dazu ist es erforderlich, Werkstoffproben aus verschiedenen Baujahren, Bauteilen und Bauwerken zu untersuchen. Bei der Auswertung der ermittelten Bruchzähigkeiten $K_{J c}$ nach dem MasterCurve-Konzept werden auch bereits zur Verfügung stehende Werkstoffdaten aus früheren Untersuchungen an alten Stählen einbezogen, um die großen Streuungen der Werkstoffeigenschaften von Flussstahl zu erfassen. Die analysierten Werkstoffproben werden begleitend durch Kerbschlagbiegeversuche untersucht, um die Bruchzähigkeit von Flussstahl hinsichtlich einer möglichen Korrelation zur Kerbschlagarbeit ähnlich der ÜbergangstemperaturKorrelation in DIN EN 1993-1-10:2010 zu prüfen. Durch Analyse der chemischen Zusammensetzung des untersuchten Probenmaterials erfolgt eine Einordnung der Flussstähle zu den 
typischen Stahlherstellungsverfahren. Außerdem wird die Bruchzähigkeit der alten Stähle hinsichtlich einer Abhängigkeit vom Herstellungsverfahren untersucht.

Bei der Bewertung der Sprödbruchgefahr mit Hilfe der Bruchmechanik spielt neben der Bauteilgeometrie die angenommene Lage und Länge einer rissähnlichen Schädigung im Bauteil eine entscheidende Rolle. Diese Annahme eines Risses wird im Fall quasi-statischer Zugbeanspruchung primär von den Werkstoffeigenschaften in unmittelbarer Umgebung der Lochränder beeinflusst. Prozessbedingt durch das Frischen mit Luft besitzen insbesondere die im Konverter hergestellten Stähle (Bessemer- und Thomasstahl) einen relativ hohen Stickstoffgehalt und neigen durch das starke Diffusionsvermögen von Stickstoff zu der als Alterung bezeichneten Versprödung. Sie ist besonders ausgeprägt im Bereich plastischer Verformungen, die z. B. bei der Herstellung der Löcher von Nietverbindungen durch Stanzen auftreten. Um das Anrissverhalten quasi-statisch beanspruchter Flussstahlbauteile, die Werkstoffeigenschaften im Bereich der Lochränder und deren Zusammenhänge zur Größe der Risse (siehe Abbildung 1.1) zu untersuchen, werden mechanische und metallografische Untersuchungen an Bauteilen mit gestanzten Löchern durchgeführt.
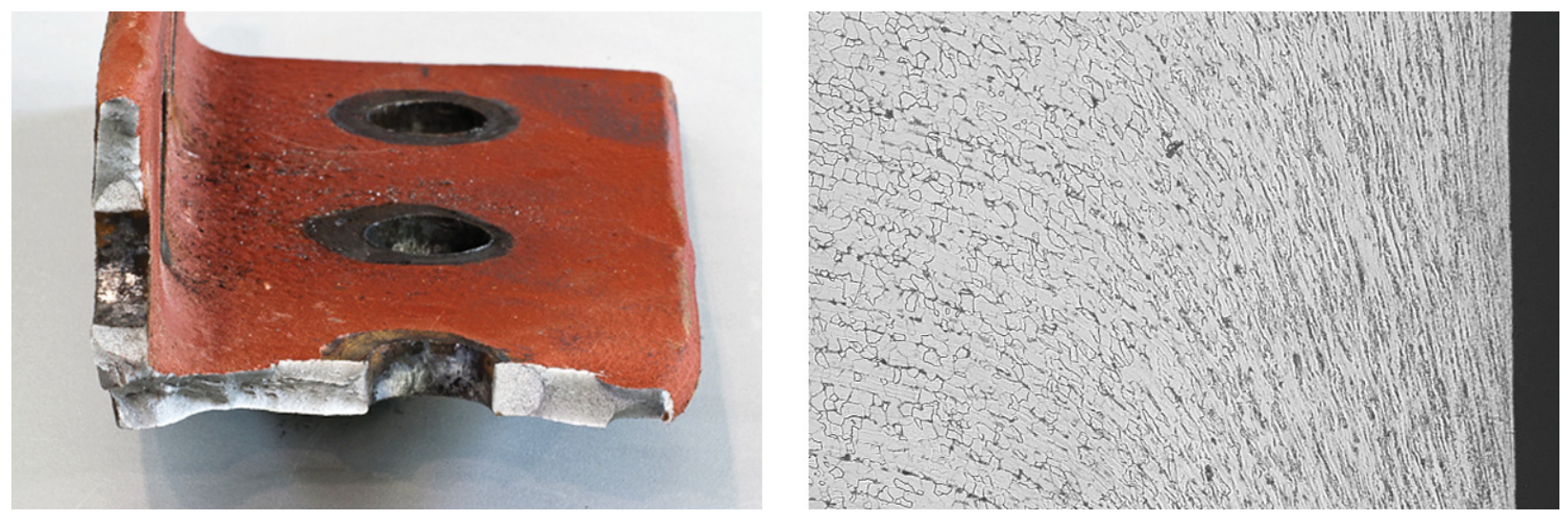

Abb. 1.1.: Spröde Anrisse an den Rändern gestanzter Löcher (links), Deformation des Werkstoffgefüges durch das Stanzen (Mikroschliff, rechts)

Da in gängigen Kompendien, wie z. B. Murakami (1990) keine bruchmechanischen Modelle zur Quantifizierung der Zähigkeitsanforderungen in exzentrisch zugbeanspruchten Winkelprofilen existieren, werden im Rahmen dieser Arbeit bruchmechanische Berechnungen mit Hilfe der FEM durchgeführt, um diese für die Bewertung von Fachwerk-Konstruktionen des Stahlhochbaus entscheidende Lücke zu schließen. Die dabei gewonnenen Ergebnisse werden anschließend in Gleichungen zur Bestimmung der erforderlichen Werkstoffzähigkeit zusammengefasst. Durch eine systematische Analyse der konstruktiven Ausbildung verschiedener bestehender Stahlfachwerkkonstruktionen des Hochbaus werden typische Querschnitte und Anschlusskonfigurationen für die numerische Analyse identifiziert.

Ausgehend von den Ergebnissen der Werkstoffuntersuchungen und numerischen Berechnungen wird ein ingenieurmäßig anwendbares Bewertungsverfahren auf semi-probabilistischer Basis erarbeitet, das dem Anwender die Möglichkeit gibt, auf Basis statistisch abgesicherter Bruchzähigkeiten von alten Baustählen eine Sicherheitsbeurteilung durchzuführen. Die Überprüfung der Vorhersagegenauigkeit des zugrunde liegenden Berechnungsmodells erfolgt durch Vergleiche mit Zugversuchen an unterschiedlichen Bauteilen des Stahlhochbaus. 


\section{Kapitel 2.}

\section{Stand der Wissenschaft}

\subsection{Entwicklung der Stahlerzeugung im 19. und 20. Jahrhundert}

Das aus Eisenerz erschmolzene, hoch kohlenstoffhaltige Roheisen kann weder geschweißt noch geschmiedet sondern nur vergossen werden. Für die Herstellung schmiedbaren Eisens sind eine Verringerung des Kohlenstoffgehaltes sowie eine Reduktion weiterer Stahlbegleiter erforderlich. Dieser Vorgang wird als das Frischen bezeichnet. Abhängig davon, ob der hierbei erzeugte Stahl in festem bzw. teigigem Zustand erzeugt und verschweißt oder flüssig vergossen wird, bezeichnet man das Produkt als Schweiß- oder Flusseisen. Schweißeisen konnte im ersten industriellen Stahlerzeugungsverfahren, dem Puddelverfahren, hergestellt werden. Aus diesem Grund wird Schweißeisen auch Puddelstahl genannt. Nachdem ab der Mitte des 19. Jahrhunderts bei der Stahlerzeugung im Konverter ausreichend hohe Temperaturen erreicht und deutlich größere Chargen in viel kürzerer Zeit hergestellt werden konnten, wurde der Puddelstahl mehr und mehr durch Flussstahl abgelöst. Während im Jahr 1887 noch etwa die Hälfte des Rohstahls in Deutschland im Puddelverfahren hergestellt wurde [VDEH (1889a)], sank bis 1913 die Produktion von Puddelstahl auf 1,3\% [Helmerich (2005)], so dass man bei Baustählen später errichteter Bauwerke mit hoher Wahrscheinlichkeit von Flussstählen ausgehen kann.

Das erste zur Herstellung von Flussstahl eingesetzte Verfahren war das 1855 in England entwickelte Bessemer-Verfahren. In diesem Prozess wurde das flüssige Roheisen in einem Konverter (Bessemerbirne) mittels Luft gefrischt, welche mit Druck vom Boden des Konverters aus durch die Schmelze geblasen wurde. Der hiermit erzeugte Flussstahl war qualitativ hochwertiger als Puddelstahl und konnte in besser reproduzierbarer Qualität erzeugt werden. Bedingt durch die saure Auskleidung des Konverters war im Bessemer-Verfahren allerdings nur Roheisen mit einem Phosphorgehalt von höchstens 0,1\% einsetzbar. Die phosphorreichen, billigen Erze aus Lothringen oder Salzgitter [REICHE (2000)] eigneten sich nicht für die Verarbeitung. Geeignetes Eisenerz bzw. Roheisen musste deshalb aus Nordspanien und England importiert werden. Somit ist der Anteil von Bauwerken aus Bessemerstahl in Deutschland vor allem nach der Jahrhundertwende eher als gering einzuschätzen. So wurden im Jahr 1906 nur etwa 4\% des in Deutschland erzeugten Rohstahls in Bessemer-Konvertern erzeugt [VDEH (1907)].

Der stetig wachsende Bedarf an Massenbaustählen konnte in Deutschland erst durch das 1878 entwickelte Thomas-Verfahren wirtschaftlich gedeckt werden. Bei dieser Methode handelt es sich um eine Weiterentwicklung des Bessemer-Verfahrens, welches durch die basische 
Auskleidung des Konverters und die Zugabe von gebranntem Kalk eine Verarbeitung des Roheisens aus phosphorreichem Eisenerz deutscher Lagerstätten ermöglichte. So nahm zwischen 1880 und 1906 die Zahl der Thomas-Konverter in Deutschland von 4 auf 105 zu und die Zahl der Bessemer-Konverter von 46 auf 14 ab [REICHE (2000), VDEH (1907)].

Zur gleichen Zeit wie das Bessemer-Verfahren wurde das Siemens-Martin-Verfahren (SMVerfahren) für die Herstellung von Qualitätsstählen entwickelt. In Deutschland führte es zuerst 1869 die Fa. Krupp in Essen ein [REICHE (2000)]. Ähnlich wie im Bessemer-Konverter konnte hier anfangs nur phosphorarmes Roheisen weiterverarbeitet werden. Am Ende des 19. Jahrhunderts gelang die Weiterentwicklung für die Nutzung phosphorreicherer Erze. Ein Vorteil des SM-Verfahrens lag in den sehr flexiblen Nutzungsmöglichkeiten des Ofens hinsichtlich der zuzuführenden Einsatzstoffe (Eisenerz, Roheisen, Schrott, Koks). Dies gestattete es, das um die Jahrhundertwende zunehmend anfallende Schrottaufkommen für die Stahlerzeugung zu nutzen.

Die Alterungsempfindlichkeit der siliziumfreien Thomasstähle infolge erhöhter Stickstoffgehalte aus dem Frischen mit Luft war bereits seit 1920 bekannt. Um dieser Materialversprödung entgegen zu wirken, wurden in der ersten Hälfte des 20. Jahrhunderts mehrere Versuche unternommen, die Eigenschaften der Konverterstähle denen des Siemens-Martin-Stahles anzugleichen. Hierfür wurde dem Wind bis zu 35 \% Sauerstoff zum Frischen hinzugegeben. Höhere Anteile führten allerdings zu einer zu starken Abnutzung der Düsen im Konverterboden durch Überhitzung [VDEH (1971)]. Der endgültige Durchbruch gelang 1952 in Österreich mit der Entwicklung des Oxygenstahl- oder Sauerstoffaufblasverfahrens (LD-Verfahren nach Linz-Donawitz), bei dem reiner Sauerstoff über eine wassergekühlte Lanze von oben auf die Stahlschmelze aufgeblasen wird [Burghardt; Neuhof (1983)]. Die Charge im Konverter muss hier jedoch zu großen Teilen aus flüssigem Rohstahl bestehen. Eine Zugabe von Schrott zum Kühlen erfolgt nur in geringen Mengen. In nicht integrierten Stahlwerken, also Werken ohne Roheisenerzeugung, in denen vorrangig Schrott und „kaltes“ Roheisen Verwendung fanden, erfolgte die Stahlherstellung somit im Siemens-Martin-Ofen oder im Lichtbogenofen (Elektro-Verfahren).

Der im Frischeprozess unverbrauchte Sauerstoff im Stahl führte zu einem „Kochen“ der Schmelze, auch nachdem diese in Kokillen vergossen worden war. Während der Erstarrung des Stahls traten durch diese fortwährenden Bewegungen in der Schmelze Entmischungserscheinungen auf, so dass die Stahlkristallite die enthaltenen Verunreinigungen wie Schwefel, Phosphor und Kohlenstoff vor sich herschoben und diese sich dadurch im Blockinneren, den sogenannten Kernseigerungen, anreicherten. Die von Verunreinigungen nahezu freien Randzonen der Blöcke werden als Speckschicht bezeichnet. Auf diese Weise unberuhigt vergossene Stähle wurden in Deutschland bis Anfang der 1930er Jahre produziert. Zur Desoxidation wie auch zum Erreichen der Festigkeit wurden dem Roheisen nach dem Frischen nur Mangan in Form von Spiegeleisen oder Ferromangan zugegeben [VDEH (1929)]. Erst in den 1930er Jahren begann man durch Legieren mit mindestens 0,15\% Silizium den überschüssigen Sauerstoff abzubinden, in die Schlacke abzuführen und den Stahl dadurch beruhigt zu vergießen [Kuscher (2012)]. Ab Mitte der 1930er Jahre desoxydierte man die Schmelze auch durch Zugabe von Aluminium [VDEH (1937)]. Man erkannte darüber hinaus, dass der Stahl bei Legierung mit Aluminium feinkörniger war, verbunden mit einer Festigkeitssteigerung ohne dass die Zähigkeit negativ beeinflusst wurde. Jedoch konnte man diesen Effekt zunächst nicht erklären. Heute weiß man, dass Aluminium zur Nitridbildung das erste feinkornbildende Element war. Damit gelang es 1939 den ersten feinkörnigen höherfesten Baustahl St52 durch Vergießen mit Aluminiumzusatz herzustellen [WeRner; SEIDEL (1992)]. 
Die hier kurz erläuterten Entwicklungen der Stahlherstellung können in Abbildung 2.1 anhand der Weltstahlerzeugung anschaulich nachvollzogen werden. Man erkennt die zunehmende Verdrängung des Bessemer-Verfahrens durch das Thomas-Verfahren und die wachsende Bedeutung des Siemens-Martin-Verfahrens. Angesichts der bereits angesprochenen Rohstoffsituation kam es in Deutschland, auch beeinflusst von politischen Ereignissen (Teilung Deutschlands nach dem 2. Weltkrieg), zu einer abweichenden Entwicklung der Stahlproduktion (siehe Abbildung 2.2).

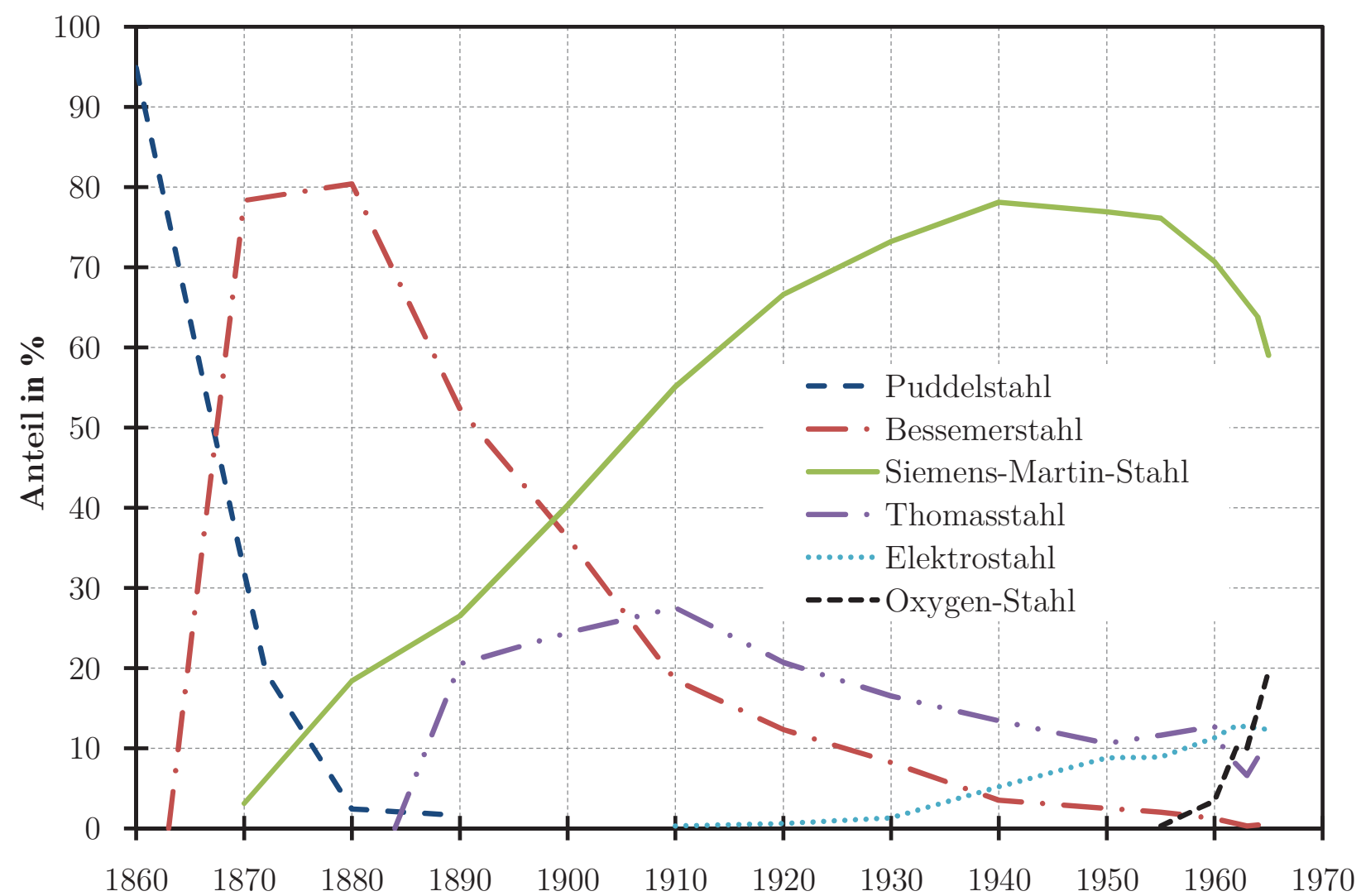

Abb. 2.1.: Entwicklung der Weltstahlerzeugung, getrennt nach Verfahren [VDEH (1971)]

Eine ausführlichere Darstellung der Entwicklung der Stahlherstellung, detaillierte Erläuterungen zur Terminologie alter Stähle sowie eine Zusammenstellung gültiger Lieferbedingungen bis in die Anfänge des 20. Jahrhunderts sind bei HeLmerich (2005) angegeben. Im Folgenden werden die Stahlherstellung und die zugehörigen normativen Regelungen in Deutschland im 20. Jahrhundert dargestellt.

\subsubsection{Deutschland in den Jahren bis 1939}

Vor 1939 wurden im Deutschen Reich ca. 5\% Elektrostähle, $40 \%$ Thomasstähle und 55\% SM-Stähle erzeugt [ECKART (1988), DröGE; BAnsen (1952)]. Die Stahlindustrie war zu dieser Zeit vor allem auf das Rheinland, Westfalen, das Siegerland und das Saargebiet konzentriert. Nur etwa 1,5\% des deutschen Roheisens und etwa 8,5\% des Rohstahls wurden 1937 im Gebiet der späteren DDR hergestellt [WIEnERT (1992)]. Um den regionalen Stahl- 
bedarf zu decken, wurden deshalb erhebliche Stahlmengen (etwa 60\%) aus dem Westen Deutschlands bezogen [ECKART (1988)].

Der einzige ostdeutsche Produktionsort für Roheisen war die Maximilianhütte in Unterwellenborn. Seit 1926 waren hier die einzigen Thomaskonverter in Betrieb. Die Stahlerzeugung in Mittel- und Ostdeutschland erfolgte vornehmlich in Siemens-Martin-Stahlwerken auf Basis des Schrotts, der in den umliegenden stahlverarbeitenden Industriezentren anfiel [WIENERT (1992)]. Die meisten Walzwerke lagen ebenso im Westen des Deutschen Reiches. Dennoch waren die Werke in Mitteldeutschland nicht unbedeutend, vor allem wegen ihres vielfältigen Produktionsprogramms.

Aus den Kriegsjahren 1939 bis 1945 existieren nur lückenhafte Daten über die Stahlerzeugung im Deutschen Reich. Es ist jedoch bekannt, dass große Teile der Produktionskapazitäten den Bedarf der Rüstungsindustrie decken mussten.

\subsubsection{Das geteilte Deutschland in den Jahren 1945 bis 1949}

Nach Ende des Zweiten Weltkrieges begannen 1945 die Besatzungsmächte mit der Demontage der nur wenig von Kriegszerstörungen betroffenen Schwerindustrie. Hiervon war besonders die Sowjetische Besatzungszone (SBZ) betroffen. Es wurden ca. $80 \%$ der vorhandenen Kapazitäten demontiert und nicht transportable Einrichtungen weitestgehend zerstört. Schon in den Jahren 1946/47 änderte jedoch die sowjetische Besatzungsmacht angesichts des sich anbahnenden Kalten Krieges ihre Strategie und beschloss den Wiederaufbau der Stahlindustrie in der SBZ. Eine Wiederaufnahme der Produktion in den Siemens-Martin-Stahlwerken Riesa, Hennigsdorf und Gröditz wie auch in mehreren angegliederten Walzwerken erfolgte in den Jahren 1947-1949 sehr zügig.

Die Produktion von Thomaseisen als Grundlage für die Stahlproduktion in 4 ThomasKonvertern und 2 Elektroöfen konnte im einzigen nicht demontierten Stahlwerk - der Maxhütte Unterwellenborn - bereits 1946 wieder aufgenommen werden. Ab 1947 erfolgte dann auch hier der Wiederaufbau der Stahl- und Walzwerke in den noch vorhandenen Restanlagen. Die Maxhütte Unterwellenborn war danach bis in die 80er Jahre das einzige integrierte Hüttenwerk auf dem Gebiet der DDR [ECKART (1988), WiEnERT (1992)].

\subsubsection{Die DDR in den Jahren 1950 bis 1970}

Das im Zuge des Wiederaufbaus errichtete Eisenhüttenkombinat Ost bei Frankfurt a.d. Oder nahm mit dem ersten von sechs Hochöfen im Jahr 1951 die Arbeit auf. Hier wurden Eisenerze und Kohle aus Polen und der Sowjetunion verarbeitet. Das geplante Stahl- und Walzwerk wurde allerdings im Jahre 1953 aus wirtschaftspolitischen Gründen gestrichen [WIENERT (1992)]. Somit musste das erzeugte Roheisen exportiert oder als „kaltes“ Roheisen zusammen mit anfallendem Schrott in den Siemens-Martin- und Elektroöfen der anderen Stahlwerke verarbeitet werden. Aus diesem Grund lag in der DDR der Anteil an SM-Stählen mit über $75 \%$ deutlich höher als in der Bundesrepublik (siehe Abbildung 2.2). Der Anteil an Thomasstahl aus den einzigen Thomaskonvertern in Unterwellenborn betrug bis in die 1970er Jahre nur ca. 8 bis $10 \%$ [ECKART (1988)]. 

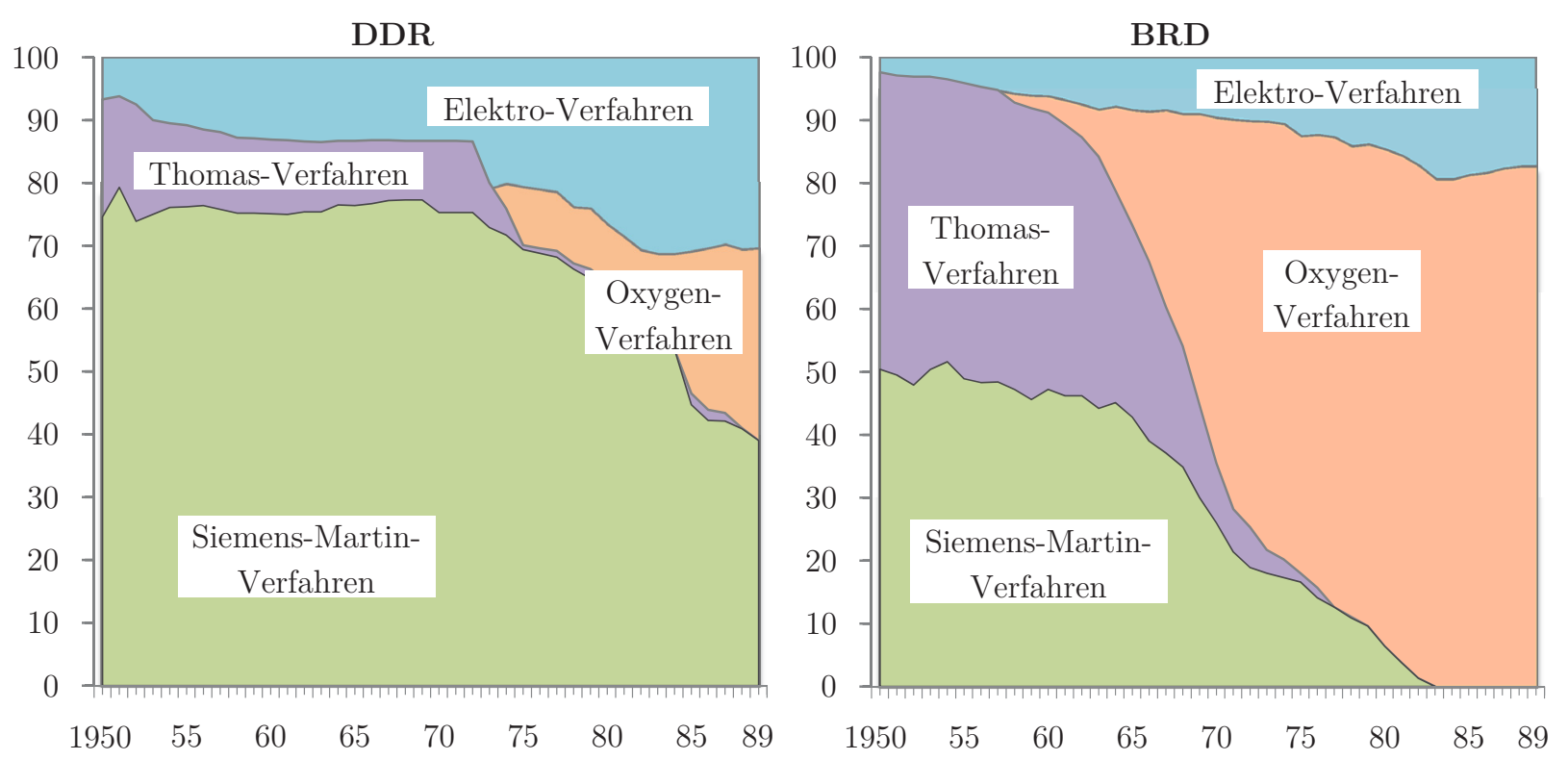

Abb. 2.2.: Anteile der Stahlerzeugungsverfahren in der DDR und der Bundesrepublik in Prozent [WiEnert (1992)]

\subsection{Entwicklung der Normung für Baustähle in Deutschland}

Im Zuge der steten Entwicklung der Eisen- und Stahlproduktion wurden auch Vorschriften und Normen erarbeitet. Eine der ersten Richtlinien dieser Art waren die 1889 veröffentlichten „Vorschriften für die Lieferung von Eisen und Stahl“ [VDEH (1889b)], welche später vor allem für den Eisenbahnbau konkretisiert wurden. Die erste relevante Vorschrift zu Baustählen in Deutschland wurde 1924 mit dem Titel „DIN 1612 - Flussstahl gewalzt“ [VDEH (1930)] herausgegeben. Für eine zweite, leicht überarbeitete Fassung erfolgte die Veröffentlichung 1932 [DNA (1941)]. Sie enthielt neben den Werkstoffeigenschaften Zugfestigkeit und zugehörige Bruchdehnung auch die Vorgabe erforderlicher Biegewinkel im sogenannten Faltversuch, der einem Nachweis der Duktilität im Lieferzustand entspricht. In der dritten Fassung der DIN 1612 im Jahr 1943 [DNA (1952)] wurden dann erste Festlegungen zur chemischen Zusammensetzung einiger Flussstähle ergänzt. Es waren jedoch noch keine Angaben zum Stickstoffgehalt enthalten (siehe Tabelle 2.1).

Dies änderte sich erst mit der Ablösung der DIN 1612 durch die „DIN 17100 - Allgemeine Baustähle“ im Jahr 1957 [DNA (1959)]. Neben einer kompletten Überarbeitung der Werkstoffbezeichnungen wurde erstmals der Maximalgehalt an Stickstoff (max. 0,01\% in der Schmelzanalyse) geregelt. Damit entsprach er nahezu den heute nach DIN EN 10025 gültigen Grenzen. Sie galten allerdings nur für in Sonderverfahren erblasene Stähle, womit zu dieser Zeit das noch recht neue Oxygenstahl-Verfahren gemeint war. Zulässige Stickstoffgehalte für Thomas- und Siemens-Martin-Stähle waren weiterhin nicht vorgeschrieben, auch wenn man in den Siemens-Martin-Öfen dieser Zeit durchaus in der Lage war, Stähle ähnlicher Qualität herzustellen.

Da die Teilung Deutschlands nach dem Zweiten Weltkrieg ebenso mit einer Trennung der 
Normung einher ging, wurden in der DDR mit der SES:1953 (Standardliste für Eisen und Stahl) und mit der TGL 7960:1960 eigene Regelungen zu Gütebedingungen allgemeiner Baustähle erarbeitet. Inhaltlich war die TGL sehr stark an die westdeutsche DIN 17100 angelehnt, so dass auch sie keine Vorgaben zum Stickstoffgehalt von Thomas- und SiemensMartin-Stählen enthielt.

Erst in der folgenden Ausgabe der TGL 7960:1975 war eine Stickstoffbegrenzung unabhängig vom Herstellungsverfahren vorgeschrieben. Ein Nachweis ausreichender Kerbschlagzähigkeit nach künstlicher Alterung wurde zwar in beiden Ausgaben beschrieben, war bei der Bestellung des Materials jedoch explizit zu fordern.

Tab. 2.1.: Werkstoffeigenschaften von Flussstählen nach DIN 1612 (1943), Auszug

\begin{tabular}{|c|c|c|c|c|c|c|c|c|}
\hline \multirow{4}{*}{$\begin{array}{l}\text { Marken- } \\
\text { bezeich- } \\
\text { nung }\end{array}$} & \multirow{4}{*}{ Güte } & \multicolumn{3}{|c|}{ Zugversuch nach DIN 1605} & \multirow{2}{*}{\multicolumn{2}{|c|}{$\begin{array}{c}\text { Faltversuch } \\
\text { nach DIN } 1605\end{array}$}} & \multirow{4}{*}{ Schweißbarkeit } & \multirow{4}{*}{$\begin{array}{l}\text { Chem. } \\
\text { Zusam- } \\
\text { men- } \\
\text { setzung }\end{array}$} \\
\hline & & \multirow{3}{*}{$\begin{array}{c}\text { Zug- } \\
\text { festigkeit }\end{array}$} & \multicolumn{2}{|c|}{ 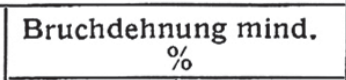 } & & & & \\
\hline & & & \multirow{2}{*}{\begin{tabular}{|c|} 
am kurzen \\
Normalstab \\
od. kurzen \\
Proportio- \\
nalstab \\
$\delta_{5}$ \\
\end{tabular}} & \multirow{2}{*}{\begin{tabular}{|c|} 
am langen \\
Normalstab \\
od. langen \\
Proportio- \\
nalstab \\
$\delta_{10}$ \\
\end{tabular}} & \multicolumn{2}{|c|}{$\begin{array}{l}\alpha=\text { Biegewinkel } \\
D=\begin{array}{l}\text { Dorndurch- } \\
\text { messer }\end{array} \\
a=\text { Probendicke }\end{array}$} & & \\
\hline & & & & & $\alpha$ & $D$ & & \\
\hline \multirow{2}{*}{ St 00.12} & \multirow{2}{*}{$\begin{array}{l}\text { Han- } \\
\text { dels- } \\
\text { güte }\end{array}$} & bis 50 & - & - & $\begin{array}{c}90^{\circ} \\
\text { kalt u. } \\
\text { warm }\end{array}$ & $\begin{array}{c}4 a \\
\text { kalt } u \text {. } \\
\text { warm }\end{array}$ & \multirow{2}{*}{$\begin{array}{l}\text { Im allgem. schmelzschweiß- } \\
\text { bar bei Form- u. Stabstahl bis } \\
25 \mathrm{~mm} \text { Dicke u. bei Breitflach- } \\
\text { stahl bis } 20 \mathrm{~mm} \text { Dicke. } \\
\text { Schmelzschweißbarkeit wird } \\
\text { aber nicht gewährleistet. }\end{array}$} & \multirow[t]{2}{*}{-} \\
\hline & & 34 bis 50 & 22 & 18 & $180^{\circ}$ & $2 a$ & & \\
\hline St 37.12 & $\begin{array}{l}\text { Nor- } \\
\text { mal- } \\
\text { güte }\end{array}$ & 37 bis 45 & 25 & 20 & $180^{\circ}$ & $0,5 a$ & $\begin{array}{l}\text { Schmelzschweißbarkeit (nach } \\
\text { Abschn. C 2) gewährleistet in } \\
\text { Thomas-Güte bei Breitflach- } \\
\text { stahl, Gurtplatten mit Steg- } \\
\text { ansatz, Nasenprofilen u. dgl., w } \\
\text { Dicke bis } 25 \mathrm{~mm} \text { beträgt, für } \\
\text { Walzerzeugnisse bis } 50 \mathrm{~mm} \text {. } \\
\text { Schmelzschweißbarkeit gewäh } \\
\text { in Siemens-Martin-Güte bei } \\
\text { erzeugnissen bis } 50 \text { mm Dick } \\
\text { Dicken über } 50 \mathrm{~mm} \text { mind über } \\
\text { währ der Schmelzschweißbark } \\
\text { sondere Vereinbarungen zu tref }\end{array}$ & $\begin{array}{l}\text { 5) } \\
\text { venn die } \\
\text { andere } \\
\text { icke. } \\
\text { rrleistet } \\
\text { Walz- } \\
\text { ke. Für } \\
\text { die Ge- } \\
\text { keit be- } \\
\text { ffen. }\end{array}$ \\
\hline St 34.12 & $\begin{array}{l}\text { Son- } \\
\text { der- } \\
\text { güte }\end{array}$ & 34 bis 42 & 30 & 25 & \multicolumn{2}{|c|}{$\begin{array}{l}\text { Die Probe muß sich } \\
\text { ohne Anrisse auf } \\
\text { der Zugseite zu zei- } \\
\text { gen, kalt zusam- } \\
\text { menbiegen lassen, } \\
\text { bis die Schenkei } \\
\text { flach aneinander- } \\
\text { liegen. }\end{array}$} & $\begin{array}{l}\text { Schmelzschweißbarkeit ge- } \\
\text { währleistet, gut feuerschweiß- } \\
\text { bar. }\end{array}$ & 5) \\
\hline
\end{tabular}

5) Für die Stähle St 37.12 u. St 34.12 wird folgende chemische Zusammensetzung gewährleistet:

\begin{tabular}{|c|c|c|c|c|c|c|c|c|}
\hline \multirow{2}{*}{$\begin{array}{c}\text { Herstell- } \\
\text { verfahren }\end{array}$} & \multicolumn{7}{|c|}{ Höchstgehalt in \% } \\
\cline { 2 - 9 } & \multicolumn{6}{|c|}{ Analyse der Schmelzung } & \multicolumn{3}{c|}{ Analyse des fertigen Werkstücks } \\
\cline { 2 - 9 } & $\mathrm{C}$ & $\mathrm{P}$ & $\mathrm{S}$ & $\mathrm{P}+\mathrm{S}$ & $\mathrm{C}$ & $\mathrm{P}$ & $\mathrm{S}$ & $\mathrm{P}+\mathrm{S}$ \\
\hline Thomas & 0,16 & 0,09 & 0,06 & 0,13 & 0,192 & 0,126 & 0,084 & 0,182 \\
\hline Siemens-Martin & 0,20 & 0,07 & 0,06 & 0,11 & 0,240 & 0,098 & 0,084 & 0,154 \\
\hline
\end{tabular}

Der offensichtliche Unterschied der TGL zur DIN war das abweichende System der Stahlmarkenbezeichnung. Um Verwechslungen bei der Beschaffung von Baustahl in den 1960er Jahren zu vermeiden, enthielt TGL 7960:1960 eine tabellarische Zusammenstellung gleichwertiger Stahlmarken in den damals gültigen Vorschriften. 


\subsection{Typische Konstruktionsformen in genieteten und geschraubten Tragwerken im Stahlhochbau}

\subsubsection{Allgemeines}

Neben den zahlreichen alten Eisen- und Stahlkonstruktionen des Brückenbaus existieren auch unzählige Konstruktionen des Stahlhochbaus, z. B. in Industrie-, Bahnhofs- und Ausstellungshallen, Masten oder Türmen. Weitaus häufiger sind Stahlbauteile in Tragkonstruktionen aber nicht sichtbar, da sie verkleidet wurden. Dazu gehören insbesondere Stützen sowie Dach- und Deckentragwerke in Industriegebäuden, Theatern oder Kaufhäusern.

Die Menge der Tragwerkstypen und die immense Anzahl an ausgeführten Konstruktionen mit ihren aus den jeweiligen Nutzungsanforderungen resultierenden Besonderheiten erschweren eine klare Darstellung von Entwicklungen und eine systematische Darstellung von Konstruktionsformen. Für die Konstruktionen des Hochbaus im Allgemeinen wurde dies bereits durch TASche (2015) und speziell für die Konstruktionen des Stahlbaus von Werner; SEIDEL (1992) aufbereitet. Eine ausführliche Analyse historischer Stahlkonstruktionen des Hallenund Industriebaus erfolgte außerdem im Rahmen des DFG-Forschungsvorhabens „Handbuch historischer Stahlhochbaukonstruktionen (1880-1940)“ [UngERmann (2008)]. Der Schwerpunkt der darin durchgeführten Untersuchungen bestand in einer systematischen Darstellung typischer Tragstrukturen und der Bestimmung von Schwachpunkten hinsichtlich ihrer Tragfähigkeit. Eine Zusammenstellung typischer Konstruktionsdetails wurde nicht erarbeitet.

Im Rahmen dieser Arbeit werden nur die Konstruktionsformen und Bauteile dargestellt, die infolge ihrer konstruktiven Ausführung sowie ihrer Beanspruchung für die Bewertung der Sprödbruchsicherheit relevant sind. Ausführlicher werden dabei die Konstruktionstypen analysiert, deren konstruktive Gestaltung für die numerischen Untersuchungen in Kapitel 4 von Bedeutung sind.

\subsubsection{Vollwandträger}

Abhängig vom Entwicklungsstand der Walzwerke der jeweiligen Zeit wurden vollwandige Träger mit I-Querschnitt und Querschnittshöhen von maximal 400 bis $600 \mathrm{~mm}$ eingesetzt. Eine Erhöhung der Biegesteifigkeit durch Aufnieten von zusätzlichen Lamellen an den Gurten erfolgte nur selten und auch nur am jeweiligen Druckgurt, da zunächst der Querschnittsverlust infolge der Lochung der sehr kräftigen Gurte durch die Lamelle kompensiert werden musste [GÖBEL; HenKel (1920)].

Für Vollwandträger mit einer Höhe von 600 bis 1500 mm wurden im Hochbau genietete Blechträger verwendet. Sie besaßen i.d. R. einen I-förmigen Querschnitt, der aus einem vertikalen Stegblech mit 8 bis $12 \mathrm{~mm}$ Dicke und Gurten aus Winkelprofilen zusammengesetzt war. Als Gurtwinkel setzte man gleich- oder ungleichschenklige Winkelprofile mit einer Schenkelbreite von mindestens $65 \mathrm{~mm}$ ein. Je nach Beanspruchung erhielten die Gurte eine Verstärkung durch zusätzlich aufgenietete Gurtlamellen (auch Deck- oder Gurtplatten genannt). Die Dicke der Lamellen variierte zwischen 10 und $14 \mathrm{~mm}$ und orientierte sich meist an der Dicke der Winkelprofile. Der verwendete Nietdurchmesser entsprach etwa dem Doppelten der Winkelprofildicke und lag zwischen 20 und 26 mm [GöBEL; Henkel (1920)]. 


\subsubsection{Fachwerkträger}

Für Dach- oder Deckenträger größerer Stützweite (ab etwa 16 m) oder bei hoher Belastung war der Einsatz von höheren Blechträgern aus optischen, wirtschaftlichen wie auch aus statischen Gründen nicht mehr sinnvoll. In diesen Fällen konstruierte man Fachwerkträger, in denen das Stegblech in ein Netz aus senkrechten und / oder geneigten Füllstäben aufgelöst wurde. Die Form der Fachwerkträger orientierte sich neben der Beanspruchung vor allem an der Funktion des Trägers (z. B. Dachbinder, Deckenträger).

Für die gedrückten Gurtstäbe des Fachwerks verwendete man überwiegend zusammengesetzte Querschnitte aus Blechen und Winkel- oder U-Profilen. Bei den schlankeren Füllstäben wurden Doppelwinkel und U-Profile teilweise mit Spreizung und einer Kopplung durch Bindebleche eingesetzt. Obgleich als Querschnitt für die Zugstäbe des Fachwerks der Flachstahl am günstigsten und optisch unauffälligsten gewesen wäre, setzte man auch hier Winkel- oder U-Profile ein. Zu groß war die Gefahr, dass die Flachstähle bei Transport oder Montage deformiert werden konnten.

Fachwerke waren so zu konstruieren, dass „alle Stabachsen sich scharf in einem Punkt, dem theoretischen Knotenpunkt schneiden, und dass die Schwerelinien der Stäbe (Stabachsen) mit den Linien des Trägernetzes zusammenfallen." [GöBEL; Henkel (1920)]. Da die zweite Forderung mit an einem Schenkel angeschlossenen Winkelprofilen jedoch nicht zu erfüllen war, galten in diesen Fällen folgende Regeln: Für kleine Winkelprofile, die einreihig mit Nieten angeschlossen wurden, war die Nietrisslinie auf der Systemlinie anzuordnen. Bei größeren Winkeleisen mit zweireihiger Nietung wurde die der Schwerelinie näher liegende Risslinie in der Netzlinie angeordnet (siehe Abbildung 2.3). Der Durchmesser der am Anschluss der Stäbe verwendeten Niete sollte etwa doppelt so groß sein, wie die mittlere Profil- oder Blechdicke der im Knoten anzuschließenden Bauteile. Bleıch (1932) gibt für den zu wählenden Nietdurchmesser die Richtwerte in Tabelle 2.2 an.
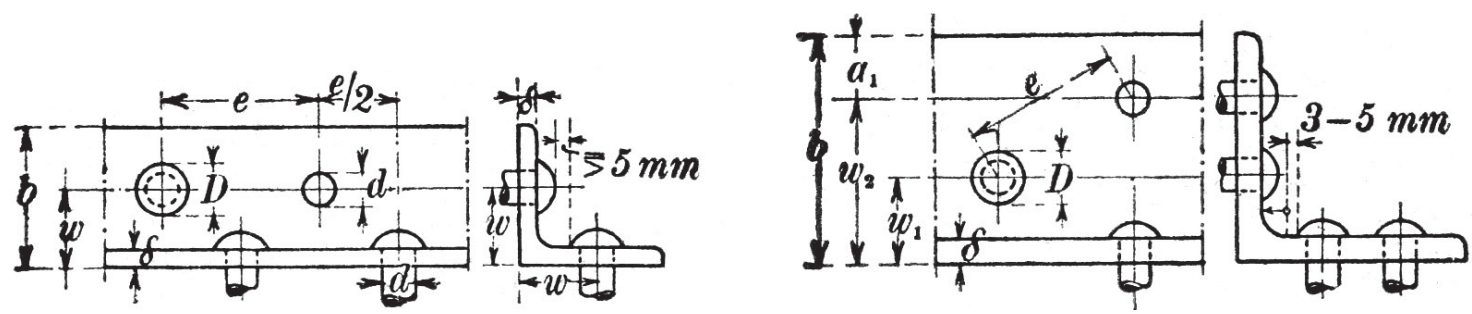

Abb. 2.3.: Nietanordnung bei schmalen (links) und breiten (rechts) Winkelprofilen nach GöBEL; HENKEL (1921)

Unabhängig von der im Anschluss zu übertragenden Kraft forderte GöBEL; Henkel (1920): „Jeder Anschluss muss aber mindestens zwei Niete erhalten, auch wenn die Berechnung weniger ergibt. („Ein Niet ist kein Niet!“)“ Um eine ungleiche Verteilung der anzuschließenden Kraft zu minimieren, wurde die Anzahl der in Kraftrichtung hintereinander angeordneten Niete auf 4 begrenzt. Für den Anschluss größerer Kräfte sollten daher mehrreihige, symmetrische Nietbilder konstruiert werden. Die Anschlüsse von Winkelprofilen waren durch Beiwinkel am abstehenden Schenkel zu verstärken. Deren Anschlussniete wurden um jeweils einen halben Nietabstand in Längsrichtung versetzt angeordnet (siehe Abbildung 2.3). Detaillierte Richtwerte zur Lage der Risslinien und dem größten zulässigen Nietdurchmesser $d$ im Anschluss von Winkelprofilen enthält Tabelle 2.3. 
Tab. 2.2.: Nietdurchmesser in Abhängigkeit der Dicke der zu verbindenden Bauteile nach BLEICH (1932)

\begin{tabular}{l|ccccc}
\hline Materialdicke $[\mathrm{mm}]$ & $4-5$ & $6-7$ & $8-10$ & $11-13$ & $14-18$ \\
\hline Nietdurchmesser $[\mathrm{mm}]$ & 14 & 17 & 20 & 23 & 26 \\
\hline
\end{tabular}

Tab. 2.3.: Wurzelmaße und Nietdurchmesser bei Winkelprofilen nach GöBEL; Henkel (1921)

\begin{tabular}{|c|c|c|c|c|c|c|}
\hline \multirow{2}{*}{$\begin{array}{l}\text { Schenkel- } \\
\text { breite } \\
\text { b[mm] }\end{array}$} & \multirow{2}{*}{$\begin{array}{l}\text { Wurzelmaß } \\
\text { w [mm] }\end{array}$} & \multirow{2}{*}{$\begin{array}{l}\text { Größter } \\
\text { zulässiger } \\
\text { Nietloch- } \\
\text { durch- } \\
\text { messer } \\
d[\mathrm{~mm}]\end{array}$} & \multirow{2}{*}{$\begin{array}{l}\text { Schenkel- } \\
\text { breite } \\
\text { b[mm] }\end{array}$} & \multicolumn{2}{|c|}{ Wurzelmaß } & \multirow{2}{*}{$\begin{array}{l}\text { Größter } \\
\text { zulässiger } \\
\text { Nietloch- } \\
\text { durch- } \\
\text { messer } \\
d[\mathrm{~mm}]\end{array}$} \\
\hline & & & & $w_{1}[\mathrm{~mm}]$ & $w_{2}[\mathrm{~mm}]$ & \\
\hline 45 & 25 & 13 & 110 & 50 & 75 & 26 \\
\hline 50 & 30 & 13 & 120 & 50 & 85 & 26 \\
\hline 55 & 30 & 16 & 130 & 50 & 95 & 26 \\
\hline 60 & 35 & 16 & 140 & 55 & 105 & 26 \\
\hline 70 & 40 & 20 & 150 & 60 & 110 & 26 \\
\hline 80 & 45 & 23 & 160 & 60 & 115 & 26 \\
\hline 90 & 50 & 23 & 200 & 65 & 150 & 26 \\
\hline 100 & 55 & 26 & & & & \\
\hline
\end{tabular}

Um eine ausreichende Lochleibungstragfähigkeit der Niete zu gewährleisten, waren bestimmte Mindestabstände der Niete untereinander und vom Bauteilrand einzuhalten. Erste Regeln für Blechstärken und Nietabstände wurden 1866 von J. W. Schwedler angegeben [WITTEK (1964)]. So gab er für den Abstand $e$ der Nietlochmitten in Kraftrichtung einen Mindestwert von $3 d$ an. GöBel; Henkel (1921) forderte bereits das Maß $3 d$ bis $4 d$. Nur in Ausnahmefällen sollte von den Grenzwerten 2,5d bzw. 5d Gebrauch gemacht werden. Der Mindestrandabstand in Kraftrichtung wurde mit $2 d$, senkrecht dazu mit mindestens 1,5d festgelegt. Ein Maß von 2,5d oder dem 7-fachen der Blechdicke durfte nicht überschritten werden, um ein Klaffen der Bauteile nach dem Nieten zu vermeiden. Detailliertere Angaben zu Regelnietabständen gibt BlEICH (1932) in Tabelle 2.4.

Um die in der Fachliteratur angegebenen Konstruktionsmaße für Anschlüsse von Winkelprofilen zu validieren, wurden im Rahmen dieser Arbeit typische Anschlussdetails in Fachwerkkonstruktionen analysiert und katalogisiert. Die Analyse umfasste mehrere tausend Anschlussdetails aus verschiedenen Konstruktionen der Baujahre 1900 bis 1940, u. a. die Dachtragwerke der Hangargebäude am Flughafen Berlin Tempelhof. Zunächst wurde die Häufigkeit des Auftretens einzelner Winkelprofilquerschnitte ausgewertet. Anschließend konnte jeder Querschnittsgröße die am meisten ausgeführte Anschlusskonfiguration zugeordnet werden. Die häufigsten Winkelquerschnitte sind mit den ausgeführten Anschlussabmessungen in Tabelle 2.5 zusammengefasst. Die Richtwerte aus den Tabellen 2.3 und 2.4 wurden dabei in nahezu allen Fällen erfüllt. 
Tab. 2.4.: Regelnietabstände in Abhängigkeit vom Nietdurchmesser nach BLEICH (1932)

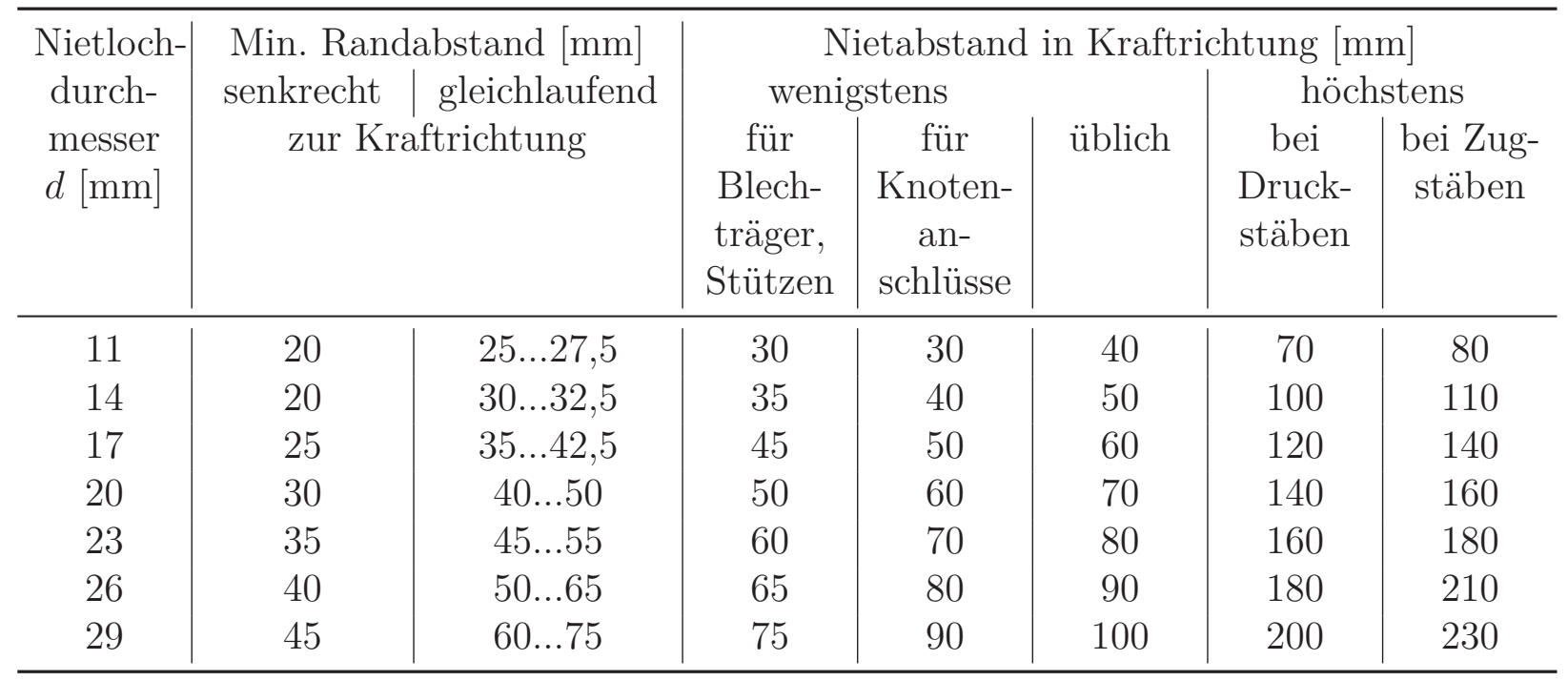

Tab. 2.5.: Nietdurchmesser sowie Rand- und Lochabstände typischer Winkelanschlüsse in Stahlfachwerkkonstruktionen nach eigenen Recherchen

\begin{tabular}{c|c|c|c|c}
\hline $\begin{array}{c}\text { Winkel- } \\
\text { schenkel- } \\
\text { breite }[\mathrm{mm}]\end{array}$ & $\begin{array}{c}\text { Nietloch- } \\
\text { durchmesser }\end{array}$ & Wurzelmaß & $\begin{array}{c}\text { Randabstand in } \\
\text { Kraftrichtung }\end{array}$ & $\begin{array}{c}\text { Nietabstand in } \\
\text { Kraftrichtung }\end{array}$ \\
\hline 45 & $d[\mathrm{~mm}]$ & $w[\mathrm{~mm}]$ & $e_{1}[\mathrm{~mm}]$ & $e[\mathrm{~mm}]$ \\
50 & 14 & 25 & $30 \ldots 35$ & $60 \ldots 100$ \\
60 & 14 & 30 & $30 \ldots 40$ & $50 \ldots 80$ \\
65 & 20 & 35 & $30 \ldots 40$ & $55 \ldots 80$ \\
70 & 20 & 45 & $40 . .50$ & $65 \ldots 140$ \\
75 & 20 & 40 & $35 \ldots 40$ & $55 \ldots 100$ \\
80 & 20 & 45 & $30 \ldots 65$ & $70 \ldots 120$ \\
90 & 20 & 50 & $43 \ldots 50$ & $70 \ldots 90$ \\
100 & 20 & 55 & $30 \ldots 70$ & $65 \ldots 100$ \\
\hline
\end{tabular}

\subsubsection{Dach- und Hallentragwerke}

Betrachtet man die historischen Stahlhochbaukonstruktionen, fällt auf, dass die Dach- und Hallentragwerke zu großem Teil aus genieteten Fachwerkbindern bestehen, die auf vollwandigen oder fachwerkartigen, eingespannten Stützen gelenkig gelagert sind. Die heute im Hallenbau übliche Konstruktionsweise des Zweigelenkrahmens wurde bis in die 30er Jahre aufgrund des erheblichen Aufwandes für die Herstellung eines genieteten, biegesteifen Rahmeneckstoßes vermieden (siehe Abbildung 2.4). Erst mit der Einführung des Fügeverfahrens Schweißen konnte eine biegesteife Eckverbindung zwischen Stütze und Riegel konstruktiv einfacher gelöst werden [BLEICH (1933)]. Diese Entwicklungen wurden auch durch die Untersuchungen von Ungermann (2008) bestätigt. Der zugehörige Abschlussbericht enthält eine detaillierte, 
chronologische Darstellung der Entwicklungen der Konstruktionen im Stahlhallenbau zwischen 1880 und 1940.

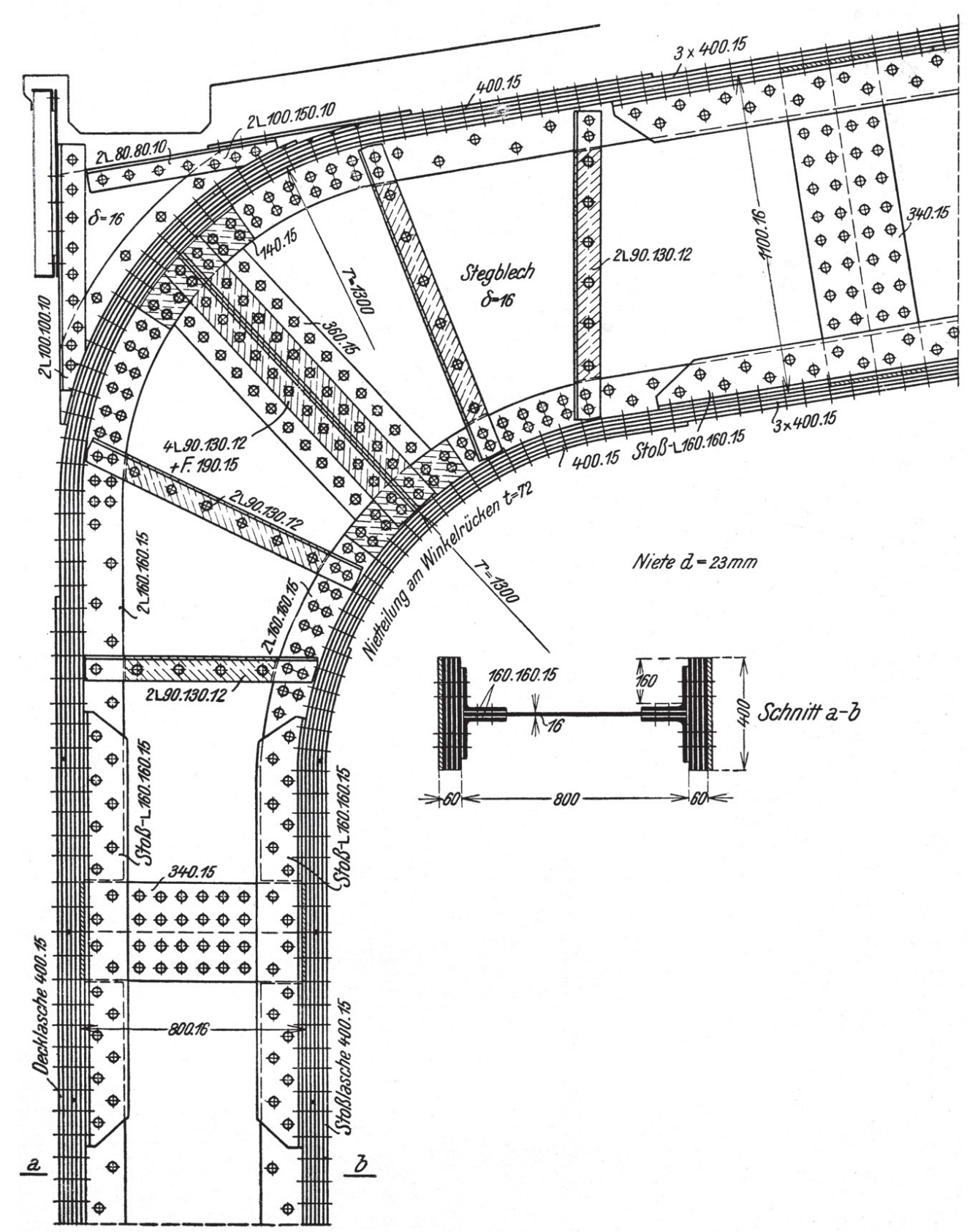

Abb. 2.4.: Genietete Rahmenecke eines großen Rahmenbinders nach BleICH (1933)

\subsubsection{Stahlgeschossbauten}

Während in anderen Ländern mehrgeschossige Stahlkonstruktionen (meist aus gusseisernen Stützen und schmiedeeisernen Trägern) bereits seit Mitte des 19. Jahrhunderts errichtet wurden, begann man mit dem Stahlskelettbau in Deutschland erst zögerlich in den 1920er Jahren [WERnER; SEIDEL (1992)]. Für die Stützen und Riegel wurden gewalzte I-Querschnitte eben- 
so wie genietete Blechträgerquerschnitte eingesetzt. Um die oben bereits genannten Schwierigkeiten bei der Konstruktion einer genieteten, biegesteifen Rahmenecke zu umgehen und eine ungehinderte Kreuzung von Stützen und Riegeln zu ermöglichen, wurden die Stützen meist zweiteilig ausgebildet. Die Riegel wurden durch die Zwischenräume in den Stützen geführt und die Auflagerkräfte und Anschlussmomente als vertikales Kräftepaar über Knaggen und Futterkeile in die Stützen übertragen (siehe Abbildung 2.5).

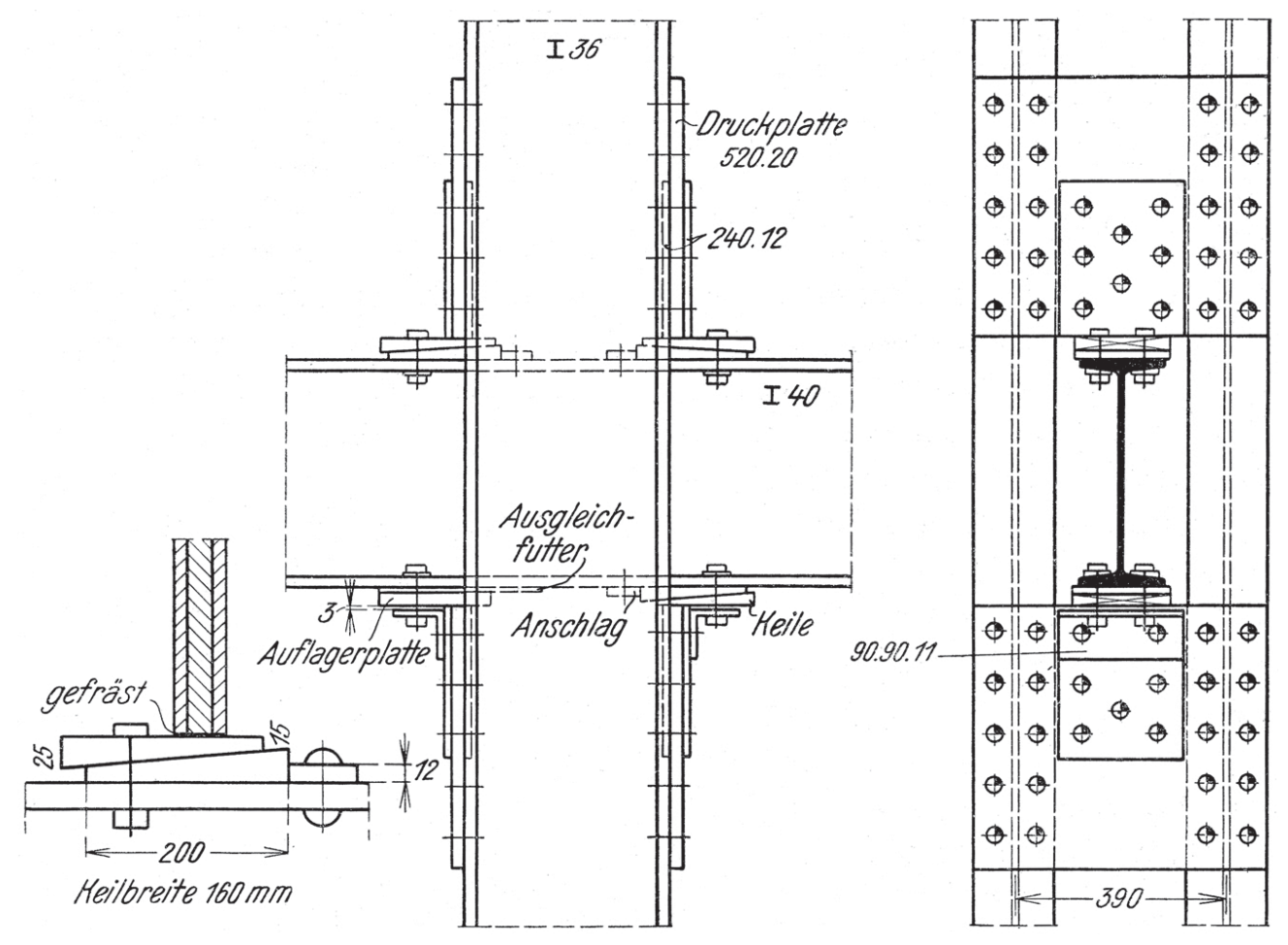

Abb. 2.5.: Genieteter biegesteifer Anschluss eines Deckenträgers an eine Geschossstütze nach BLEICH (1932)

\subsubsection{Leitungsmaste und Funktürme}

Die im Allgemeinen als Stahlgittermasten bezeichneten Konstruktionen der Leitungsmaste und Funktürme waren durch eine meist sehr schlanke Stahlfachwerkkonstruktion gekennzeichnet, deren Form und Abmessungen sich vor allem an ihrer Beanspruchung und einer möglichst einfachen Konstruktionsweise orientierten. So bestanden sie i. d. R. aus vier Fachwerkwänden in quadratischer Anordnung im Grundriss mit einer Neigung von $3 \%$ bis $5 \%$ zur Reduzierung des Querschnitts zum Mastkopf hin. Für die Eckstiele wie auch für die Ausfachung wurden nahezu ausschließlich kleinere bis mittlere Winkelprofile verwendet. Entgegen der Meinung von GöBeL; Henkel (1920): „Ein Niet ist kein Niet!“ wurden die Winkelprofile in Stahlgittermasten und Verbindungsniete so gewählt, dass ein Anschluss der Diagonalen am Eckstiel mit nur einem Niet und vor allem ohne zusätzliches Knotenblech möglich war. Abbildung 2.6 zeigt übliche Ausführungsvarianten solcher Anschlüsse.

Um die Stabkräfte hoch beanspruchter Diagonalen auch mit nur einem Niet übertragen zu können, ließ z. B. die ab 1930 gültige Vorschrift für den Bau von Starkstromleitungen deutlich größere Nietdurchmesser zu (Tabelle 2.6), als sie im Stahlhochbau üblich waren (Tabelle 2.3). 

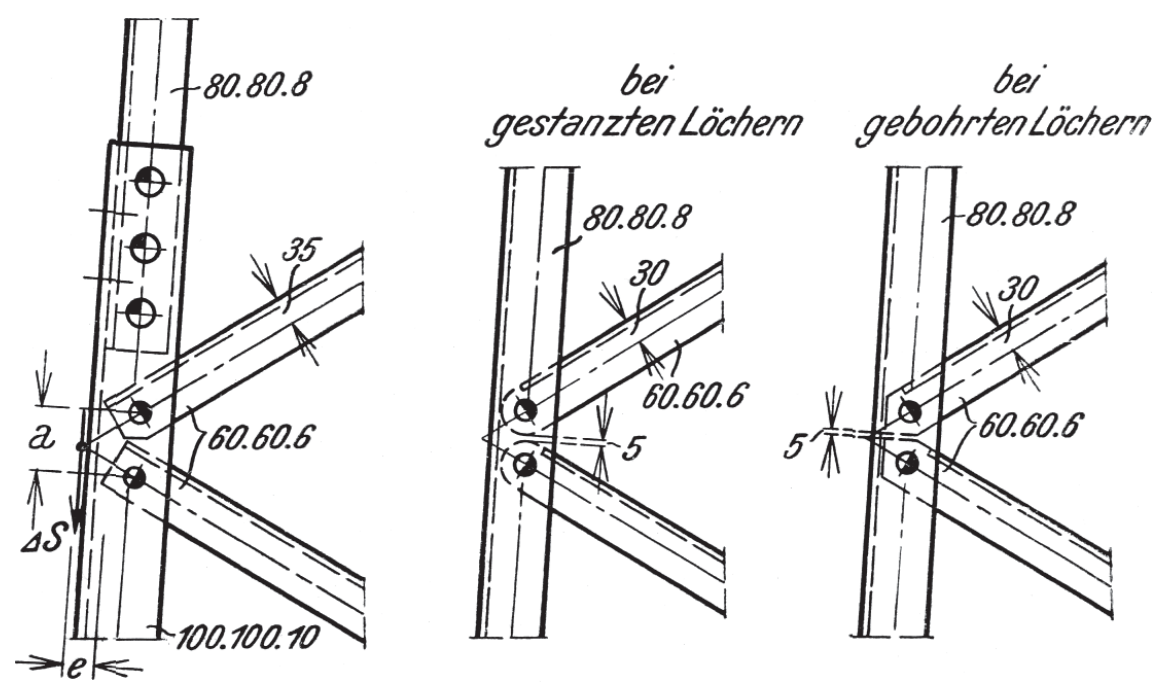

Abb. 2.6.: Anschlüsse der Diagonalen an den Eckstielen von Stahlgittermasten nach BLEICH (1933)

Damit verbunden waren außerdem sehr kleine Randabstände der Niete, die das Mindestmaß von $1,5 d$ bis $2 d$ nicht selten unterschritten.

Tab. 2.6.: Nietdurchmesser in Abhängigkeit der Winkelschenkelbreite in Stahlgittermasten BLEICH (1933)

\begin{tabular}{l|lllllll}
\hline Winkelschenkelbreite $[\mathrm{mm}]$ & 40 & 45 & 50 & 60 & 70 & 75 & 80 \\
\hline max. Nietdurchmesser $[\mathrm{mm}]$ & 14 & 16 & 17 & 20 & 23 & 26 & 29 \\
\hline
\end{tabular}

Um das Versatzmaß a der Stabachsen im Knoten (siehe Abbildung 2.6) zu minimieren, wurden die Stabenden der Diagonalen abgeschrägt oder abgerundet. Letztere Form wurde, wie auch die Nietlöcher selbst, meist durch Stanzen erzeugt. Die Folgen dieser Bearbeitungsmethode in Verbindung mit den geringen Randabständen der Verbindungsmittel auf das Bruchverhalten der Bauteile werden in Abschnitt 2.4 erläutert.

Um für diesen Sonderfall der mit einem Niet angeschlossenen Winkelprofile die in der Fachliteratur angegebenen Konstruktionsmaße zu validieren, wurden im Rahmen dieser Arbeit typische Anschlussdetails von Diagonalen in Stahlgittermasten analysiert und katalogisiert. Die Analyse umfasste knapp 12.000 Anschlussdetails aus 87 verschiedenen Leitungsmasttypen der Baujahre 1900 bis 1960. Zunächst wurde die Häufigkeit einzelner Winkelprofilquerschnitte ausgewertet. Anschließend konnte jeder Querschnittsgröße die am meisten ausgeführte Anschlusskonfiguration zugeordnet werden. Die sechs häufigsten Winkelquerschnitte, die ca. $70 \%$ aller untersuchten Details entsprechen, sind mit ihren typischen Anschlussabmessungen in Tabelle 2.7 zusammengefasst. Es ist zu erkennen, dass die maximalen Nietdurchmesser nach Tabelle 2.6 nur in der Hälfte der Fälle ausgenutzt wurden. Der Randabstand $e_{1}$ in Kraftrichtung liegt aufgrund der oft schlanken Eckstielquerschnitte mit bis zu 1,25d deutlich unter den Vorgaben in Tabelle 2.4. 
Tab. 2.7.: Nietdurchmesser und Randabstände typischer Winkelanschlüsse in Stahlgittermasten [FREITAG (2013)]

\begin{tabular}{c|c|c|c}
\hline $\begin{array}{c}\text { Winkel- } \\
\text { querschnitt }\end{array}$ & $\begin{array}{c}\text { Nietloch- } \\
\text { durchmesser } \\
d[\mathrm{~mm}]\end{array}$ & $w[\mathrm{~mm}]$ & $\begin{array}{c}\text { Randabstand in } \\
\text { Kraftrichtung } \\
e_{1}[\mathrm{~mm}]\end{array}$ \\
\hline L40 x & 12 & 22 & 20 \\
L50 × 5 & 16 & 30 & 20 \\
L60 × 6 & 20 & 35 & 25 \\
L70 × 7 & 23 & 40 & 30 \\
L80 × 8 & 26 & 45 & 35 \\
L90 x & 26 & 50 & 40 \\
\hline
\end{tabular}

\subsection{Das Bruchverhalten unlegierter Baustähle}

\subsubsection{Bruchmechanismen und makroskopisches Bruchverhalten}

Bei der Beschreibung des Bruchverhaltens ferritischer Stähle mit kubisch-raumzentrierter Gitterstruktur wird grundsätzlich zwischen den mikroskopischen Bruchmechanismen Spaltbruch und Gleitbruch unterschieden. In Metallen mit kubisch-flächenzentriertem Gitter (z. B. austenitische Stähle) tritt nur Gleitbruch auf [GRÄFEN (1991)].

Der Spaltbruch, der auch als instabiles Risswachstum bezeichnet wird, kann transkristallin oder interkristallin verlaufen. Der transkristalline Spaltbruch entsteht durch die Trennung von Kristallebenen, wenn die Kohäsionskräfte der Atome überwunden werden. Eingeleitet wird er durch lokale Spannungskonzentrationen, die durch einen Aufstau von Versetzungen an Korngrenzen oder Einschlüssen entstehen können. Die maßgebende Voraussetzung für die Entstehung eines Spaltbruches ist die Überschreitung der Spaltbruchfestigkeit an der Störstelle über eine kritische Distanz. Spaltbruch führt mikroskopisch zu einer glatten, durch einzelne Körner verlaufenden Bruchfläche (siehe Abbildung 2.7 rechts). Die makroskopische Bruchfläche erscheint kristallin glänzend [ВӧHм (1992)].

Im Unterschied dazu basiert der Gleitbruch auf plastischen Fließvorgängen im Werkstoff. Diese entstehen durch das Abgleiten von Versetzungen auf kristallografischen Ebenen. Bei technischen Werkstoffen, wie den Baustählen, verläuft ein Gleitbruch infolge plastischen Fließens, ausgehend von Störstellen im Gitter, durch die Bildung, das Wachstum und die anschließende Vereinigung von Hohlräumen [Böнm (1992)]. Durch das die Werkstofftrennung herbeiführende Abscheren der Verbindungen zwischen den Hohlräumen erscheint die Bruchfläche mikroskopisch betrachtet wabenförmig (siehe Abbildung 2.7 links). Makroskopisch erscheint sie stattdessen matt. Der Gleitbruch wird auch als stabiles Risswachstum bezeichnet, da für ein Voranschreiten der Rissfront eine Lastzunahme erforderlich ist [LANGENBERG (1996)].

Der Spaltbruch tritt generell bei tieferen Temperaturen auf als der Gleitbruch. Der Übergang von einer Bruchart zur anderen erfolgt kontinuierlich in einem gewissen Temperaturbereich. In diesem Übergangsbereich treten beide Bruchmechanismen gemeinsam auf. Ausgehend von tiefen Temperaturen tritt ab einer bestimmten Temperatur erstmals Gleitbruch in Bereichen 

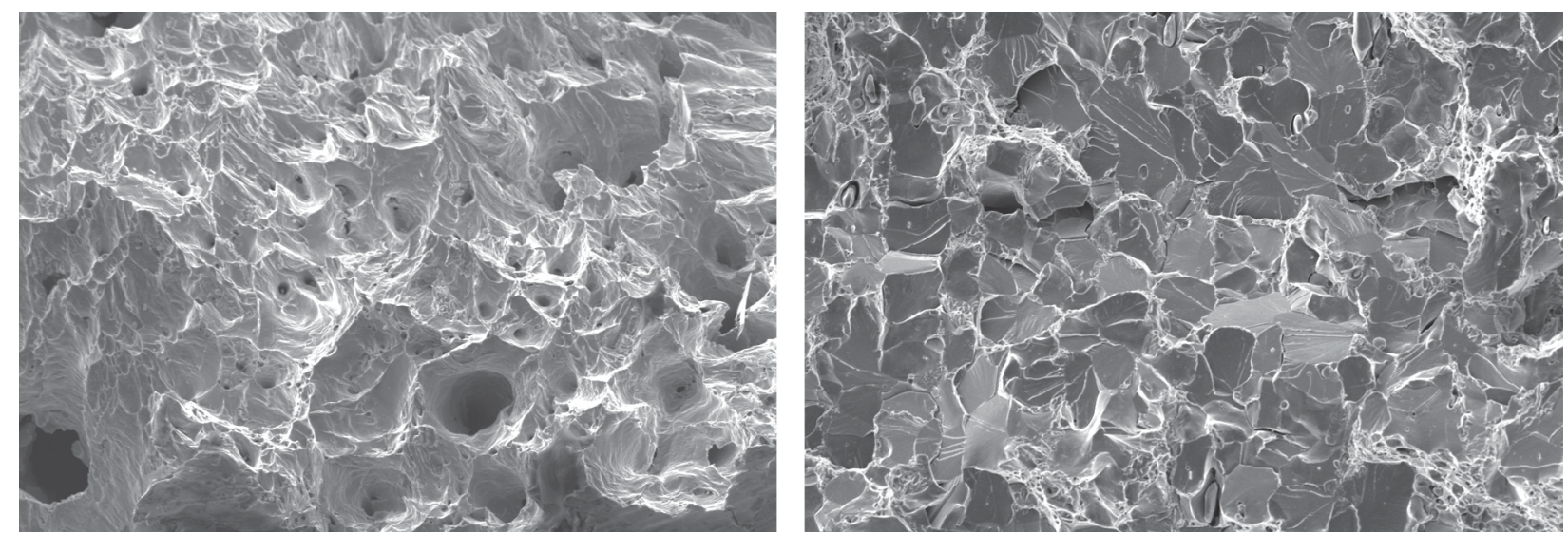

Abb. 2.7.: Gleitbruch mit Waben und z.T. Einschlüssen (links), Transkristalliner Spaltbruch (rechts), 1000-fach

maximaler Kerbspannung (z. B. an einer Rissspitze) auf. Mit steigender Temperatur nimmt der Gleitbruchanteil kontinuierlich zu. Ein Bruchvorgang im Übergangstemperaturbereich erfolgt generell so, dass die Werkstofftrennung zunächst als Gleitbruch beginnt und mit zunehmender Laststeigerung spontan in den Spaltbruch übergeht [SchatT; Simmchen et al. (1998)].

Das makroskopische Bruchverhalten des Werkstoffs wird darauf aufbauend anhand des Verformungszustandes im Moment des Bruches beschrieben. Bei einem linear-elastischen Materialverhalten und spontanem Versagen durch Spaltbruch spricht man von Sprödbruch. Weist das Bauteil jedoch ein elastisch-plastisches Werkstoffverhalten auf und versagt mit sichtbaren, plastischen Verformungen, so wird dies als Zähbruch bezeichnet.

\subsubsection{Verfestigung durch Alterung}

Unter dem Begriff der Alterung wird im Allgemeinen eine Veränderung des Gefüges eines metallischen Werkstoffes verstanden, wobei diese immer auch mit Änderungen der mechanischen Eigenschaften verbunden ist. Die Ursache sind Ausscheidungsvorgänge aus übersättigten Mischkristallen des Werkstoffs.

Es wird generell zwischen natürlicher und künstlicher Alterung unterschieden. Während natürliche Alterung im Baustahl von selbst auftritt, kann im Unterschied dazu die Alterung auch durch eine Wärmebehandlung herbeigeführt bzw. beschleunigt werden. In diesem Fall spricht man von künstlicher Alterung. Im Allgemeinen ist mit dem Begriff Alterung die nach einer Kaltverformung eintretende Reckalterung gemeint, die besonders für Stahl von Bedeutung ist. Verantwortlich für diesen Alterungsvorgang sind neben Kohlenstoff, Phosphor und Sauerstoff insbesondere der gelöste, nicht abgebundene Stickstoff, weil dieser das größte Diffusionsvermögen besitzt [Schatт; Simmchen et al. (1998)]. Immer wenn die Schmelze bei der Stahlherstellung mit Luft in Kontakt kommt, also beim Frischen, Abstechen und Vergießen, kann Stickstoff aufgenommen werden. Während die flüssige Stahlschmelze bis zu 0,027\% N aufnehmen kann und die maximale Löslichkeit von Stickstoff in Eisen bei $590{ }^{\circ} \mathrm{C}$ etwa $0,10 \%$ beträgt, sinkt sie auf nur 0,014\% bei Raumtemperatur [BAEHRE; KÄPPLEIN (1990)]. Die N-Atome werden dadurch während der Abkühlung im Eisen zwangsweise gelöst, 
obwohl für sie kein Platz im Gitter ist. Diese derart übersättigte Lösung hat das Bestreben, im Lauf der Zeit in einen stabilen Zustand zu gelangen. Folglich diffundieren die ungebundenen Stickstoffatome langsam zu den Korngrenzen hin. Auf dem Weg dort hin passieren sie kleine Leerstellen oder Versetzungen im Gitter, in denen sie eingelagert werden können. Dies führt zu einer Verzahnung der Gitterstruktur, damit zu einer Erhöhung der Streckgrenze und Zugfestigkeit bei gleichzeitig sinkender Verformbarkeit und Zähigkeit des Stahls [Reiche (2000); Schatt; Simmchen et al. (1998)].

Bereits um die Jahrhundertwende wurde der Einfluss des Stickstoffs auf die Eigenschaften der Stähle untersucht und eine Festlegung maximal zulässiger Werte unter den Fachleuten kontrovers diskutiert [BRAunE (1906)]. Wenige Jahre später unternahm STROMEYER (1910) einen ersten Versuch, den Einfluss von Stickstoff im Vergleich zu Phosphor auf die Versprödung von Stahl zu quantifizieren. Auch er forderte, den Stickstoffgehalt bei der Bewertung der Sprödigkeit des Materials zu berücksichtigen und schlug vor, „dass der Phosphorgehalt zusammen mit dem fünffachen Betrage des Stickstoffgehaltes 0,08\% nicht überschreiten soll“. Es wurden darauf hin erste Versuche unternommen, den Stickstoff durch Legieren mit Titan abzubinden. Ein Abbinden durch das Legieren mit Aluminium geschah erstmals, wenn auch nur als Nebeneffekt, Ende der 1930er Jahre im Zuge der Entwicklung höherfester Baustähle [Werner; SEIDEL (1992)]. Durch technologische Weiterentwicklung des Thomas-Verfahrens gelang es nach dem 2. Weltkrieg, durch Anreicherung der zum Blasen eingesetzten Luft mit bis zu einem Drittel Sauerstoff, den Stickstoffgehalt im erzeugten Stahl zu reduzieren. Der Durchbruch gelang mit der Entwicklung der Oxygenstahl-Verfahren in den 1950er Jahren (siehe Abschnitt 2.1).

\subsubsection{Sprödbruchgefährdung gelochter Stahlkonstruktionen}

Die Gefahr eines Sprödbruches in genieteten oder geschraubten Stahltragwerken wird vorrangig durch werkstoffspezifische, konstruktionsbedingte wie auch fertigungsbedingte Einflüsse bestimmt. So wirkt sich im Hinblick auf eine verstärkte Alterungsneigung der erhöhte Stickstoffgehalt der windgefrischten Konverterstähle (Bessemer- und Thomas-Verfahren) nachteilig aus. Die Gefahr der damit verbundenen Sprödbruchanfälligkeit infolge Reckalterung nimmt vor allem im Bereich plastischer Verformungen enorm zu. Diese treten insbesondere bei Kaltverformung des Materials auf. Fertigungsbedingt können solche Verfestigungen z. B. im Randbereich gestanzter Löcher auftreten.

Während Anfang des 20. Jahrhunderts in ermüdungsbeanspruchten Konstruktionen gestanzte Löcher nur in sekundären Bauteilen erlaubt waren (siehe DIN 120-2:1936), wurden die Nietlöcher für Verbindungen in Stahlhochbaukonstruktionen im 19. Jahrhundert fast ausschließlich „gestanzt, weil zunächst das Bohren zehn mal so teuer kam“ [WiTTEK (1964)]. In der „Hütte - des Ingenieurs Taschenbuch“ [HANEMANN (1911)] war bereits zu lesen: „Das Lochen ist das billigere Verfahren und setzt gutes Eisen von genügender Zähigkeit voraus. [...] Flußeisen soll nur gebohrt werden."

Die Stanzung von Nietlöchern, z. B. beim Bau von Stahlgitter-Freileitungsmasten, war gemäß VDE 0210 (siehe BLEICH (1933)) bis zu einer Materialstärke von 8 mm zugelassen. Später ging man sogar dazu über, auch die Enden der Verbandsstäbe (vorrangig Winkelprofile) bis zu einer Materialstärke von $12 \mathrm{~mm}$ mit der Schlagschere zu schneiden. Aber auch Bauteile in 
Stahlbrücken (siehe MöLL (1997)) wurden durch Stanzprozesse bearbeitet. Die Auswirkungen dieser Fertigungsmethoden auf die Sprödbruchneigung wurden u. a. von SteinhardT (1954), Naumann (1961), Helms; Kühn et al. (1973) und Mors (1980) untersucht.

Die beim Stanzen hervorgerufenen Kaltverformungen führen in Verbindung mit einem zur Alterung neigenden Stahl zur Versprödung des Materials am Bauteil- oder Lochrand. Als konstruktionsbedingte Kerben verursachen Löcher Spannungsspitzen, die für ein Erreichen der rechnerischen Tragfähigkeit des Bauteils durch lokale Plastizierung abgebaut werden müssen. Ist dieses örtliche Fließen des Stahls aufgrund von Versprödung nicht in ausreichendem Maße möglich, kommt es im Anschlussbereich bei Zugbeanspruchung zu einem verformungsarmen Versagen ohne Vorankündigung vor Erreichen der rechnerischen Bruchlast.

Die Reckalterung gestanzter Bauteil- und Lochränder lässt sich, wenn nicht bereits optisch durch Deformationen am Bauteil sichtbar, metallografisch durch Mikrohärtemessungen oder durch eine Ätzung nach Fry [GRÄFEN (1991)] am Makroschliff nachweisen. Hierbei werden Kraftwirkungslinien durch Eisennitridausscheidungen in den gittergestörten Zonen des Gefüges als dunkle Streifen sichtbar gemacht (siehe Abbildung 2.8).

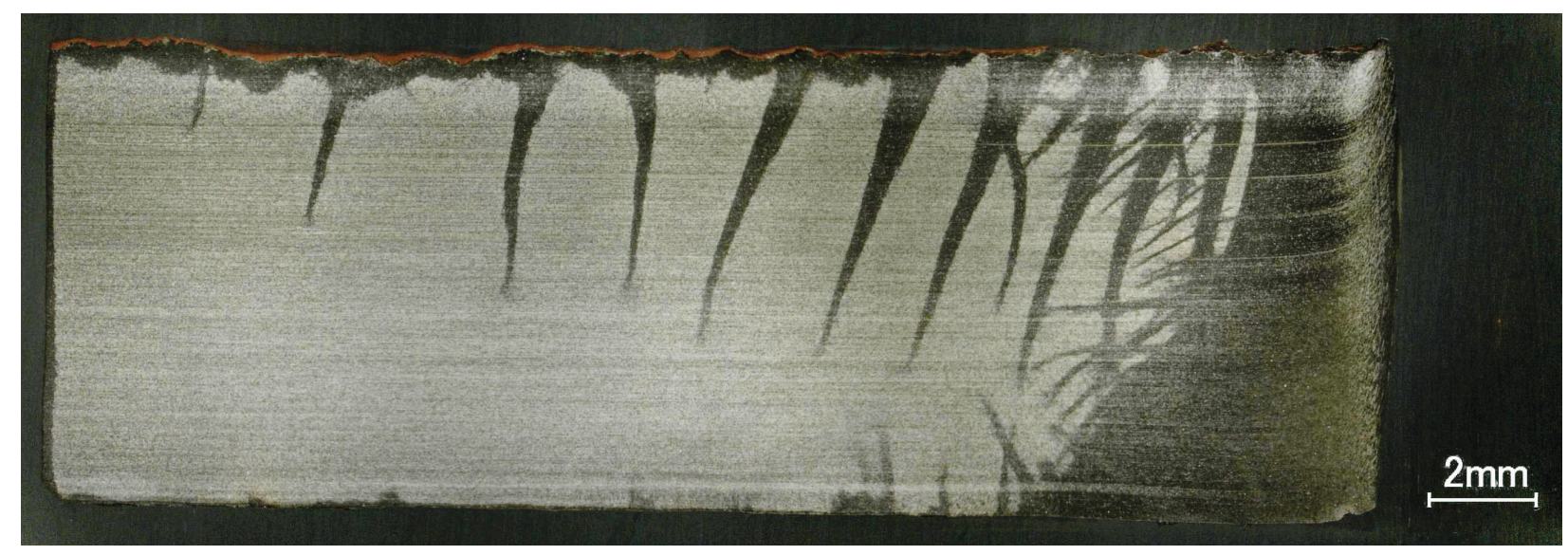

Abb. 2.8.: Kraftwirkungslinien an einem gestanzten Lochrand in einer Makroätzung nach Fry

Auch im Stahlbau des 21. Jahrhunderts stellt das Stanzen eine nicht wegzudenkende wirtschaftliche Alternative zum Bohren oder Sägen dar. Während das Problem der Reckalterung bei heutigen Baustählen kaum noch eine Rolle spielt, beeinflusst die Kaltverfestigung des Materials weiterhin dessen Tragfähigkeit, Ermüdungsfestigkeit und Sprödbruchneigung. Deshalb beschäftigen sich auch jüngere Untersuchungen wie z. B. von HuHN (2004) oder BRown; Lubitz et al. (2007), mit dem Einfluss des Stanzens, insbesondere dort, wo es durch die derzeitige Normung noch eingeschränkt oder untersagt ist. DIN EN 1090-2:2011 lässt gestanzte Kanten und Löcher nur in quasi-statisch beanspruchten Konstruktionen zu und gibt in Tabelle 10 eine maximale Härte von 380 HV10 an. Diese Angabe liegt in der gleichen Größenordnung von 350 HV10 in Schulze (2010), ab dem das Eintreten von Sprödbruch wahrscheinlich wird. Die vor dem Eurocode im Stahlbau gültige DIN 18800-7:2008 legte fest: „Bei gescherten Schnitten und gestanzten Ausklinkungen in zugbeanspruchten Bauteilen sind die neben dem Schnitt befindlichen, beschädigten und verfestigten Zonen in den Schnittflächen abzuarbeiten, es sei denn, dass durch das Schweißen diese Zonen aufgeschmolzen werden." 


\subsection{Konzepte der Sprödbruchsicherheitsnachweise}

\subsubsection{Sprödbruchsicherheit nach DIN EN 1993-1-10}

Die Vermeidung von Sprödbruch in neuen Stahlkonstruktionen erfolgt gegenwärtig durch die Wahl einer erforderlichen Stahlgütegruppe nach DIN EN 1993-1-10:2010, deren Abschnitt 2 inhaltlich weitestgehend identisch mit der DASt-Ri. 009:2008 ist. Kriterien für die Wahl der Stahlgütegruppe sind u. a. die Einsatztemperatur, das Spannungsniveau, die Festigkeitsklasse des Stahls und die Bauteildicke. Die Stahlgütegruppe wird durch die Einhaltung der Mindestkerbschlagzähigkeit unter einer vorgegebenen Prüftemperatur nachgewiesen. Die zulässigen Erzeugnisdicken einer Gütegruppe für bestimmte Einsatzbedingungen (siehe z. B. Tabelle 2.1 in DIN EN 1993-1-10:2010) wurden auf der Grundlage der Bruchmechanik ermittelt.

Bei der bruchmechanischen Methode wird unter den für das jeweilige Bauteil geltenden Einsatzbedingungen und der Annahme eines rissartigen Fehlers der Bemessungswert der Zähigkeitsanforderung (Spannungsintensitätsfaktor $K_{I}$ ) bestimmt. Liegt dieser unterhalb des Bemessungswertes der Bruchzähigkeit $K_{I c}$ des eingesetzten Werkstoffes, kann von einer ausreichenden Sicherheit gegen Sprödbruch ausgegangen werden.

Die generelle Vorgehensweise in DIN EN 1993-1-10:2010 ist in Abbildung 2.9 illustriert und wurde von SEDlaceK; Feldmann et al. (2008) ausführlich dokumentiert. In Teilbild a) ist das bruchmechanische Modell dargestellt. Ausgehend von einem blechdickenabhängigen Ausgangsfehler (semi-elliptischer Oberflächenriss der Tiefe $a_{0}$, z. B. eine „unfallartig“ bei üblichen Fertigungskontrollen übersehene Ungänze) wird für 500.000 Lastwechsel ein kritischer Fehler mit der Tiefe $a_{d}$, die sogenannte Bemessungsfehlergröße, ermittelt. Diese Lastwechselzahl entspricht dem Zeitraum, in dem das an der kritischsten Stelle „fiktiv unfallartig“ geschädigte Bauteil auch bei einem Anwachsen der Schädigung unter häufigen Lasten und einer extremen Tieftemperatur sicher betrieben werden kann. Brückenbautypische Intervalle zur grundlegenden Erneuerung des Korrosionsschutzes liegen heutzutage bei etwa einem Viertel der Lebensdauer, die rechnerisch mit 2 Millionen Lastwechseln angesetzt wird. Ausgehend von der maximalen Fehlergröße am Ende des Betrachtungszeitraums wird die Rissspitzenbeanspruchung $K_{I}$ im Bauteil ermittelt. Der die Beanspruchung erhöhende Einfluss ausgebreiteter plastischer Zonen wird durch die Grenzkurve des Failure Assessment Diagrams der R6-Routine [ZERBST; Wiesner et al. (1999)] (siehe Teilbild b) berücksichtigt. Da in den aktuellen technischen Lieferbedingungen für Baustähle keine Bruchzähigkeiten $K_{c}$ sondern Mindestwerte der Übergangstemperatur der Kerbschlagarbeit $T_{27 J}$ angegeben sind, wird der Risswiderstand des Werkstoffs, mit Hilfe der von Wallin eingeführten Master-Curve [WALlin (1984)] in statistisch aufbereiteter Form, in die Analyse einbezogen (Teilbild c). Die auf diese Weise aus der bruchmechanischen Beanspruchbarkeit gewonnene Übergangstemperatur $T_{100}$ (bei der der Mittelwert der Bruchzähigkeit einem Wert von $100 \mathrm{MPa} \sqrt{\mathrm{m}}$ entspricht, auch $T_{0}$ genannt) wird dann mit Hilfe der modifizierten Sanz-Korrelation [SANZ (1980)] auf die stahlbautypische Übergangstemperatur $T_{27 J}$ aus dem Kerbschlagbiegeversuch umgerechnet (Teilbild d).

Mit dieser Vorgehensweise wird die Sicherheitsanalyse, unter Umgehung aufwendiger Versuche zur Bestimmung der Bruchzähigkeit, an die für den Stahlbau gebräuchlichen technischen 
a)

Bruchmechanik-Modell für Strukturelemente mit angenommenem Ausgangsdefekt

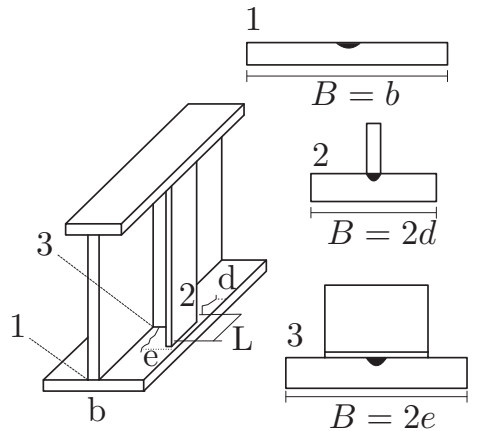

Geometrie des Ausgangsrisses: Risstiefe $a_{0}=0,5 \ln \left(t / t_{0}\right)$ $a_{0} / c=0,4$ Rissbreite $2 c=5 a_{0}$

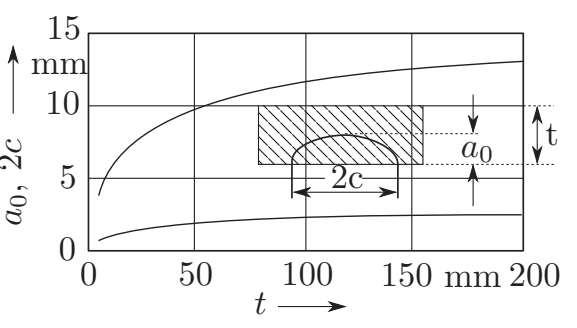

b)

Zähigkeitsanforderungen für den Grenzzustand von $K_{m a t}$

$$
K_{m a t}=\frac{K_{I}}{K_{R}}
$$

c)

Zähigkeitsanforderungen in Form einer Temperaturverschiebung $T-T_{100}$ der Master Curve

\section{CEGB-R6 Option 2}
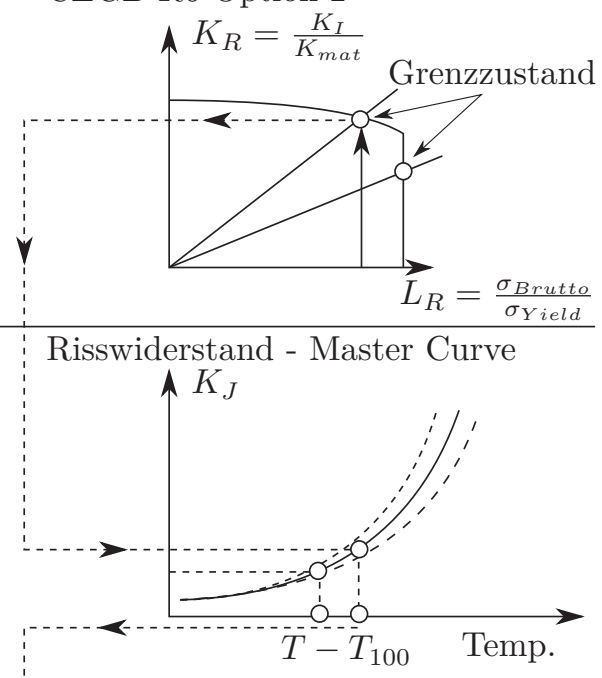

d)

Zähigkeitsanforderungen als $T_{27 J}$ Übergangstemperatur-Korrelation (mod Sanz-Korrelation)

$T_{100}=T_{27 J}-18^{\circ} \mathrm{C}$ $\sigma=13^{\circ} \mathrm{C}$

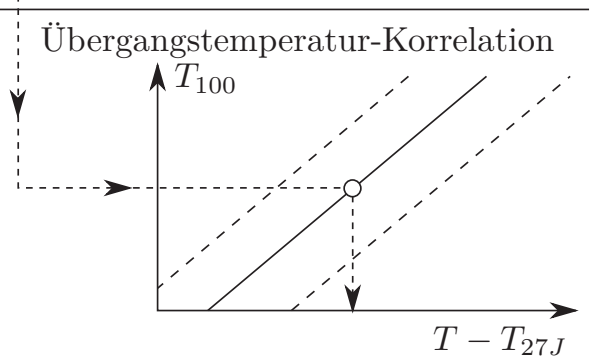

Abb. 2.9.: Bruchmechanische Grundlagen beim Sprödbruchsicherheitsnachweis im Eurocode 3 [SEDlaceK; Feldmann et al. (2008)]

Lieferbedingungen mit den darin garantierten Mindestwerten der Kerbschlagzähigkeit angebunden. Im Ergebnis dieser Berechnungen erfolgt die Werkstoffgüteauswahl u. a. in Abhängigkeit der Einsatztemperatur des Bauteils, dessen Auslastung, Bauteildicke und geplanter Werkstofffestigkeit mit Tabelle 2.1 in DIN EN 1993-1-10:2010.

Diese normativ geregelte Nachweismethode zur Beurteilung der Sprödbruchgefährdung wurde für Schweißkonstruktionen aus „Stählen aus neuerer Zeit“ mit in der Regel hohen Zähigkeitswerten entwickelt. Die Bestimmung der Werkstoffzähigkeit erfolgt durch Kerbschlagversuche unter definierten Temperaturen, die stellvertretend für die Einsatzbedingungen der Stähle stehen. Die Beziehung zwischen der Kerbschlagzähigkeit und der Bruchzähigkeit wird durch die Sanz-Korrelation hergestellt, die für diese Stähle abgeleitet wurde. Das Verfahren ist für Flussstahlkonstruktionen mit Lochschwächungen durch Niet- und Schraubenverbindungen nicht geeignet. Einerseits unterscheiden sich Kerbwirkung und Eigenspannungszustand von geschweißten und genieteten Konstruktionen und damit die Zähigkeitsanforderungen wesentlich voneinander. Auf der anderen Seite unterliegen die Zähigkeitseigenschaften von Flussstählen deutlich größeren Streuungen. Grund hierfür sind die im Vergleich zu heutigen Stählen stärker streuenden Werkstoffeigenschaften, hervorgerufen insbesondere durch höhere Gehalte an nicht-metallischen Einschlüssen.

Es ist unbedingt zu beachten, dass die im Kerbschlagbiegeversuch ermittelte Übergangstemperatur zwar ein Klassifizieren des Sprödbruchverhaltens einzelner Werkstoffe gestattet, sie aber nicht als untere Grenze der Anwendungstemperatur angesehen werden darf [BLUMENAUER (1994)]. Die Kerbschlagarbeit von 27 Joule ist eine auf Erfahrungswerten beruhende 
Größe. Sie wird deshalb im zuvor beschriebenen Sicherheitskonzept eingesetzt. Sie hat aber nur eine bedingte Aussagekraft für ein bestimmtes Werkstoffverhalten [MicheLs; HAGEDORN (1997)]. Die Kerbschlagarbeit und ihre Übergangstemperatur sind als Kriterien für die Bewertung der Sprödbruchneigung bei tiefen Temperaturen nur dann anwendbar, wenn bereits Erfahrungen hinsichtlich des Bruchverhaltens des Werkstoffs unter den geforderten Einsatzbedingungen vorliegen. So ist aus Untersuchungen von HENSEN (1992), LANGENBERG (1996) und STÖTzeL (1998) bereits bekannt, dass der im Kerbschlagbiegeversuch ermittelte Übergangsbereich bei alten Flussstählen häufig bei $+10^{\circ} \mathrm{C}$ oder noch höher liegt, die gleichen Stähle in bruchmechanischen Analysen aber eine ausreichende Werkstoffzähigkeit aufweisen.

\subsubsection{Bewertung der Sprödbruchneigung von Bauteilen in Stahlgitter-Freileitungsmasten}

Im Münsterland im Nordwesten Deutschlands führten im November 2005 starker Schneefall und kräftiger Wind zur Anlagerung von großen Mengen nassen Schnees an mehreren Freileitungen. Unter der Last dieser Schneewalzen an den Kabeln versagten mehrere Freileitungsmaste durch Knicken von Diagonalen und einige von ihnen durch Sprödbruch. Die Bundesanstalt für Materialforschung und -prüfung (BAM) wurde von der Bundesnetzagentur (BNetzA) aufgefordert, die möglichen Fehlerursachen des Bauwerkversagens zu untersuchen. Nach umfangreichen Ermittlungen vor Ort und im Labor wurde der primäre Bruch an einer zugbeanspruchten Diagonale identifiziert, die aus Thomas-Stahl bestand und die durch lokale Versprödung infolge Alterung geschwächt war [KLINGER; MEHDiAnPour et al. (2011)]. Aufgrund dieses Ereignisses und den oben genannten Untersuchungen wurde vom Verband der Elektrotechnik (VDE) die Richtlinie VDE-AR-N 4210-3:2011 zur Bewertung der Tragfähigkeit bestehender Stahlgitter-Freileitungsmaste erarbeitet.

Im Jahr 2005 existierten noch etwa 57.000 Freileitungsmaste, die bis Anfang der 70er Jahre errichtet worden waren, bevor die Herstellung von Thomas-Stahl eingestellt wurde. Bei durchschnittlich 200 Bauteilen je Mast, galten somit mehr als 11 Millionen Bauteile als potenziell sprödbruchgefährdet. Um ein Versagen der Stahlgittermaste infolge Sprödbruch mit ausreichender Sicherheit ausschließen zu können, ist es notwendig, abhängig von der Anzahl aller vorhandenen Komponenten, eine bestimmte Stichprobe an Bauteilen zu untersuchen. Entsprechend der Regelungen in VDE-AR-N 4210-3:2011 sind zugbeanspruchte Bauteile dann anfällig für sprödes Versagen, wenn sie einen Stickstoffgehalt von mehr als 100 ppm aufweisen und Kaltverformungen in Bereichen hoher Beanspruchung vorhanden sind. Da letztere nur mit größerem Aufwand nachweisbar sind, wird die chemische Zusammensetzung der in der Stichprobe verwendeten Stähle hinsichtlich des Stickstoffgehalts analysiert. Wenn die Anzahl analysierter Proben mit erhöhtem Gehalt an Stickstoff einen in der Richtlinie definierten Annahmewert überschreitet, ist die Tragfähigkeit der betreffenden Bauteile, wie von Klinger; Mehdianpour et al. (2011) vorgeschlagen, durch Bauteilzugversuche zu bestimmen. Wird durch diese Versuche eine im Vergleich zu normativen Forderungen niedrigere Beanspruchbarkeit ermittelt, müssen die betreffenden Masten einer statisch-konstruktiven Bewertung, unter Berücksichtigung der ermittelten Tragfähigkeit, unterzogen werden.

Das in VDE-AR-N 4210-3:2011 geregelte Bewertungsverfahren fordert, bevorzugt diejenigen Bauteile im Zugversuch zu untersuchen, deren Grundmaterial einen besonders hohen 


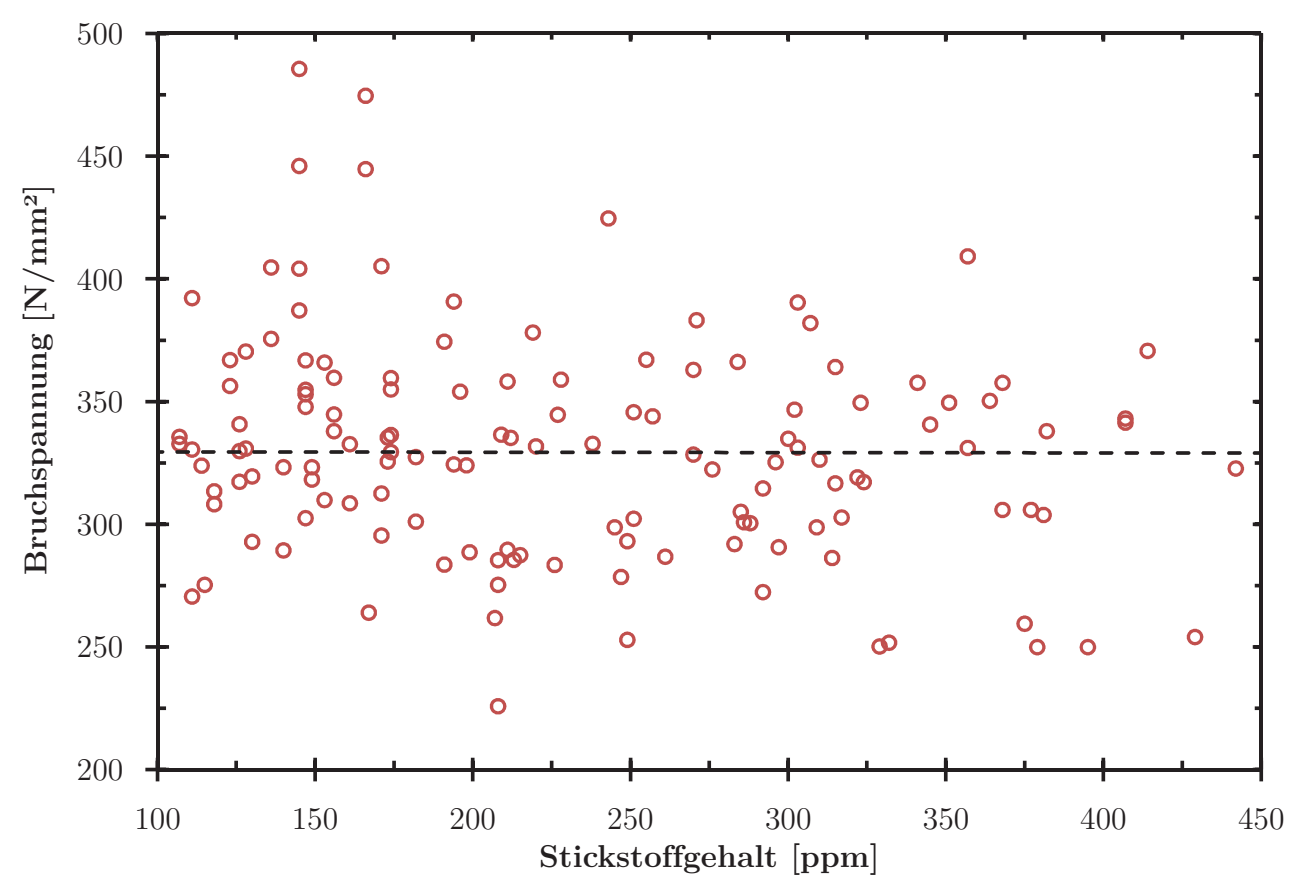

Abb. 2.10.: Stickstoffgehalte und Bruchspannungen im Bauteilzugversuch von Bauteilen aus Stahlgitter-Freileitungsmasten

Gehalt an Stickstoff aufweist. Dieses Vorgehen basiert auf der Annahme, dass diese Bauteile werkstoffbedingt eine geringere Tragfähigkeit besitzen. Wird jedoch der Stickstoffgehalt der bei solchen Untersuchungen analysierten Baustähle der Bruchlast im Bauteilzugversuch gegenübergestellt, ist keine Korrelation zwischen diesen beiden experimentellen Parametern nachweisbar (siehe Abbildung 2.10).

Die am Institut für Stahl- und Holzbau der TU Dresden experimentell ermittelten Bruchspannungen weisen eine große Streuung auf, wie sie generell für die mechanischen Eigenschaften alter Flussstähle typisch ist. Alle Bauteile, die in diesen Zugversuchen getestet wurden, versagten ausschließlich duktil. Eine wesentliche Ursache dafür ist die in VDE-AR-N 42103:2011 geforderte Durchführung der Versuche bei Raumtemperatur, obwohl die bemessungsmaßgebende Umgebungstemperatur von Freileitungsmasten bei $0{ }^{\circ} \mathrm{C}$ und darunter liegt. Bei diesen Temperaturen kann es zu der eingangs beschriebenen Bildung von Eiswalzen an den Kabeln und damit zu einer für die Tragsicherheit maßgebenden Belastungssituation kommen. Darüber hinaus ist bei tieferen Temperaturen mit weniger duktilem Werkstoffverhalten zu rechnen.

Bei weiteren Untersuchungen am Institut wurde die Tragfähigkeit konstruktiv gleichartiger Anschlüsse an zwei Hälften von Diagonalen aus Stahlgittermasten, jeweils bei Raumtemperatur und bei niedrigen Temperaturen, geprüft. Die ermittelten Traglasten unterschieden sich nur unwesentlich voneinander. Versagte ein Bauteil bei tiefen Temperaturen allerdings durch Sprödbruch, sank dessen Tragfähigkeit auf bis zu $65 \%$ gegenüber der Traglast bei Raumtemperatur (siehe Abbildung 2.11). Es ist daher dringend anzuraten, in VDE-AR-N 4210-3:2011 die Durchführung der Zugversuche bei niedrigeren Temperaturen zu fordern, um sprödes Werkstoffverhalten und eine damit verbundene Reduzierung der Tragfähigkeit der Bauteile zu identifizieren. Zusätzlich sollte die Auswahl der Bauteile für die Zugversuche nicht ausschließlich anhand des Stickstoffgehaltes erfolgen. Die Komponenten sollten stattdessen auf 
Grundlage ihrer konstruktiven Gestaltung und den damit verbundenen Anforderungen an die Werkstoffzähigkeit priorisiert werden. Eine Quantifizierung dieser erforderlichen Zähigkeit ist mithilfe bruchmechanischer Betrachtungen möglich (siehe Kapitel 2.6).

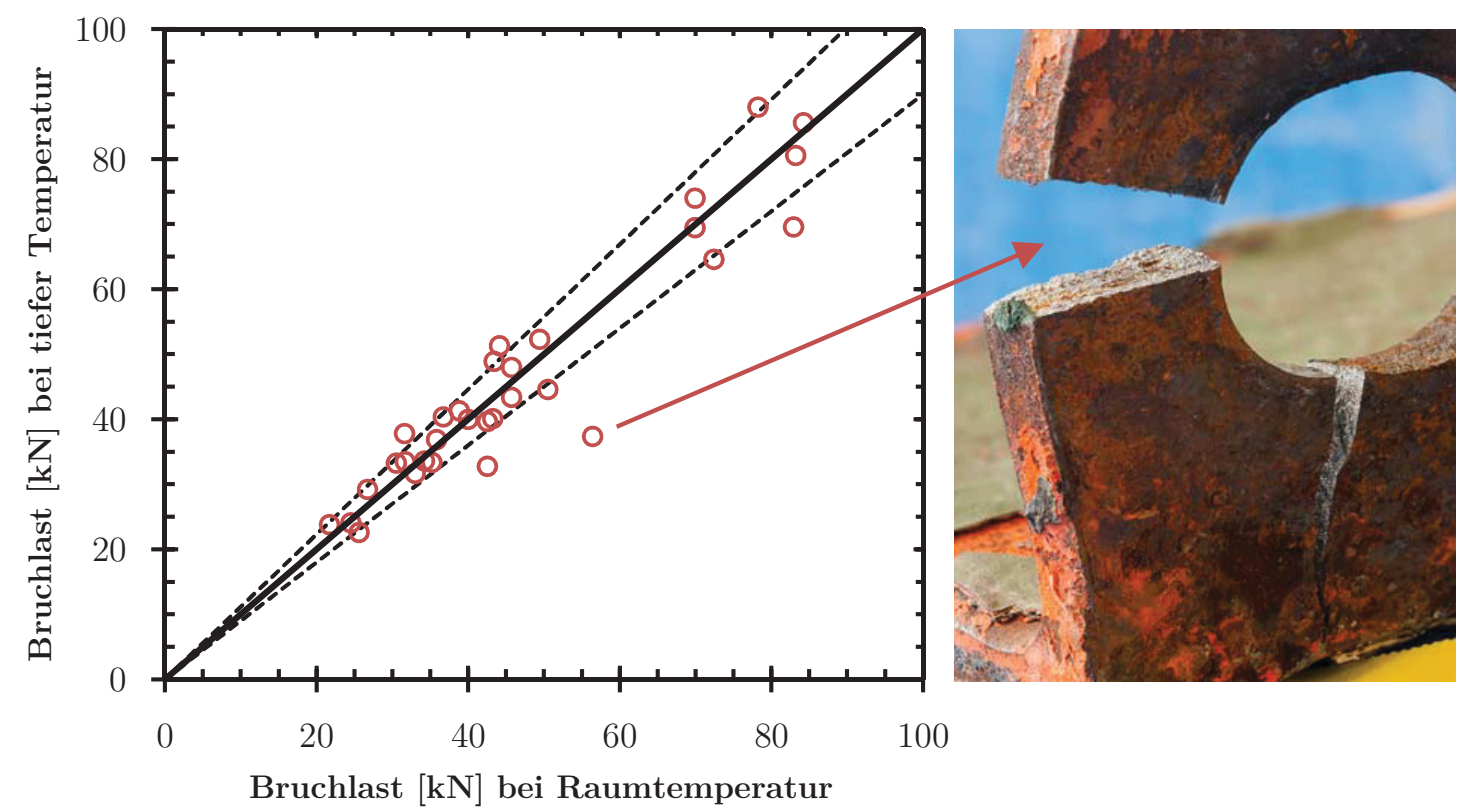

Abb. 2.11.: Bruchlasten bei verschiedenen Versuchstemperaturen, Sprödbruch am Anschluss

\subsubsection{Sprödbruchneigung geschweißter Flussstähle}

Zur Frage der Schweißbarkeit von Altstählen sind der einschlägigen Fachliteratur unterschiedlich differenzierte und praxisnahe Aussagen und Empfehlungen zu entnehmen (vgl. Mang; Bucak (1996), Möll (1997), Lüddecke (2006), Kühn; Lukić et al. (2008) und Kuscher (2012)). Flussstähle gelten als bedingt schweißgeeignet. Bekanntermaßen weisen alte Baustähle gegenüber heutigen Stählen geringere Werkstoffzähigkeiten auf. Daher ist der Sprödbruchsicherheit, wegen der durch das Schweißen verursachten Kerbwirkungen und Eigenspannungen, besondere Aufmerksamkeit zu widmen.

Um das Sprödbruchrisiko nicht unnötig zu erhöhen, wird von MANG; BuCAK (1996) und KüHN; Lukić et al. (2008) empfohlen, den Einsatz von Schweißverbindungen soweit wie möglich zu vermeiden. Wird aus konstruktiven Gründen das Schweißen dennoch angestrebt (z. B. bei Möll (1997) und Stroetmann; Flederer et al. (2009)), sind die metallurgischen Besonderheiten der Stähle zu beachten. Hierzu gehören neben den Konzentrationen an Verunreinigungen in den Seigerungszonen die Neigung zur Versprödung durch stickstoffbedingte Alterung bei windgefrischten Konverterstählen (Thomas- und Bessemer-Stahl).

Systematische Untersuchungen zur Schweißbarkeit von Flussstählen, der Auswahl und Anpassung der Schweißprozesse sowie der Sprödbruchneigung und der Ermüdungsfestigkeit der Schweißverbindungen wurden von LÜDDECKE (2006) durchgeführt. Von wesentlicher Bedeutung für eine ,altstahlgerechte“ Schweißung ist demnach, dass die Einbrandtiefe im Grundmaterial innerhalb der metallisch reineren Speckschichten der Profile verbleibt. Die Untersuchungen waren deshalb auf Kehlnähte an der Bauteiloberfläche beschränkt. Ein baustellengeeignetes Verfahren dafür ist das E-Handschweißen mit basisch umhüllten Stabelektroden. 
Der Schweißzusatz bindet die Eisenbegleiter und führt zu zähem Schweißgut. Die Verwendung kleiner Elektrodendurchmesser gewährleistet geringen Einbrand und Wärmeeintrag. Das Schweißen der Kehlnähte in Form von Strichraupen führt darüber hinaus zu einem Normalisieren der jeweils unteren Lagen und damit zu einer weiteren Reduzierung der Eigenspannungen.

Die von LüDDECKE (2006) durchgeführten Berechnungen zur Sprödbruchneigung geschweißter Bauteile aus Flussstahl basierten auf dem von KüHN (2005) beschriebenen Vorgehen in DIN EN 1993-1-10:2010 und den von LANGENBERG (1996) statistisch ausgewerteten Bruchzähigkeiten alter Baustähle. Die Gültigkeit dieser Annahmen, insbesondere für die im Stahlhochbau eingesetzten Baustähle, wird im Rahmen dieser Arbeit untersucht.

\subsection{Bruchmechanische Sicherheitsanalyse}

\subsubsection{Grundlagen der Bruchmechanik}

Bei der Beurteilung der Tragsicherheit von Bauteilen und Konstruktionen ist neben dem Fließ- und Verformungsverhalten auch das Bruchverhalten des verwendeten Werkstoffs entscheidend. Technische Bauteile weisen sehr häufig konstruktionsbedingte Kerben und herstellungsbedingte Risse auf. Die Technische Bruchmechanik bietet die Möglichkeit, durch Quantifizierung der Werkstoffzähigkeit sowie der erforderlichen Zähigkeit im Bauteil, Versagenskriterien abzuleiten. Dazu gehören die Ermittlung der kritischen Belastung oder der kritischen Rissgröße zur Vermeidung spröden Bauteilversagens.

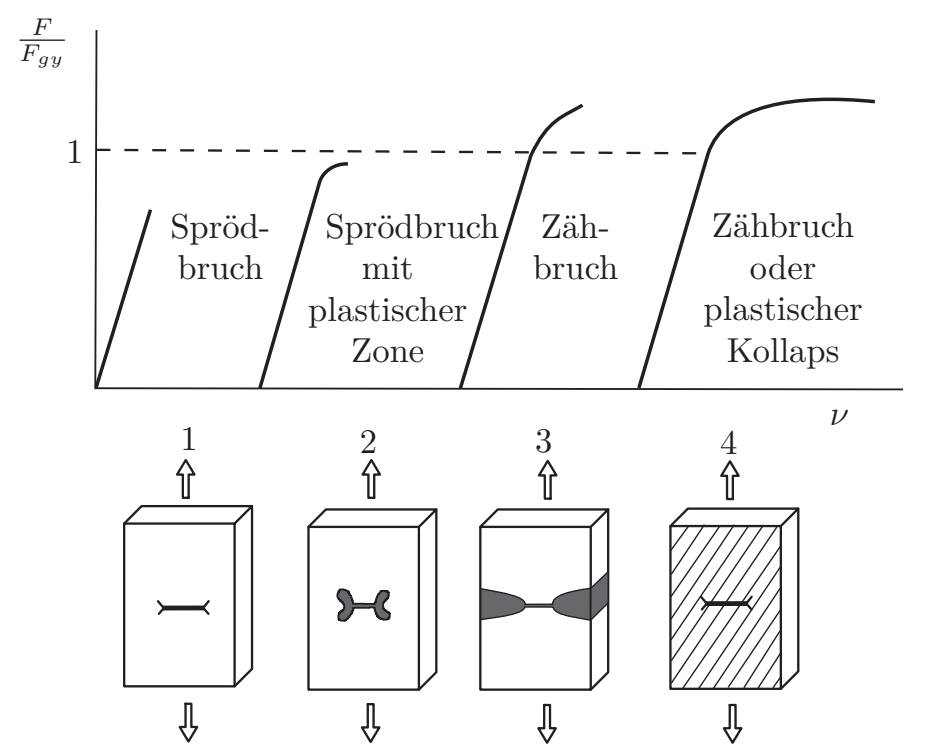

1 linear-elastische Verformung
2 Ausbildung einer begrenzten plastischen
Zone vor der Rissspitze (Kleinbereichsfließen)
3 plastische Verformung im Rissligament
4 ausgedehntes plastisches Fließen
$F$ aufgebrachte Last
$F_{g y}$ plastische Grenzlast (generell yield)
$\nu$ Aufweitung an der Rissspitze

Abb. 2.12.: Verformung eines Bauteils mit Riss bis zum Bruch [Blumenauer; Pusch (1993)]

Abhängig vom Verformungsverhalten des rissbehafteten Bauteils unter quasi-statischer Beanspruchung (schematisch dargestellt in Abbildung 2.12) wurden verschiedene Konzepte der Bruchmechanik entwickelt. Es wird zwischen der linear-elastischen Bruchmechanik (LEBM), der LEBM mit Kleinbereichsfließen und der elastisch-plastischen Bruchmechanik (EPBM) 
unterschieden, deren Grundlagen im Folgenden kurz beschrieben werden. Auf bruchmechanische Konzepte, die ein Risswachstum infolge zyklischer, thermischer oder korrosiver Beanspruchung beschreiben, wird in dieser Arbeit nicht eingegangen.

In Abhängigkeit der Beanspruchungsrichtung am Riss und den dadurch hervorgerufenen Relativbewegungen der Rissufer, unterscheidet man zwischen den in Abbildung 2.13 dargestellten Riss- bzw. Bruchmodi:

- Mode I: Normalbeanspruchung senkrecht zu den Rissufern

- Mode II: Schubbeanspruchung in Rissebene in Rissausbreitungsrichtung

- Mode III: Schubbeanspruchung in Rissebene senkrecht zur Rissausbreitungsrichtung
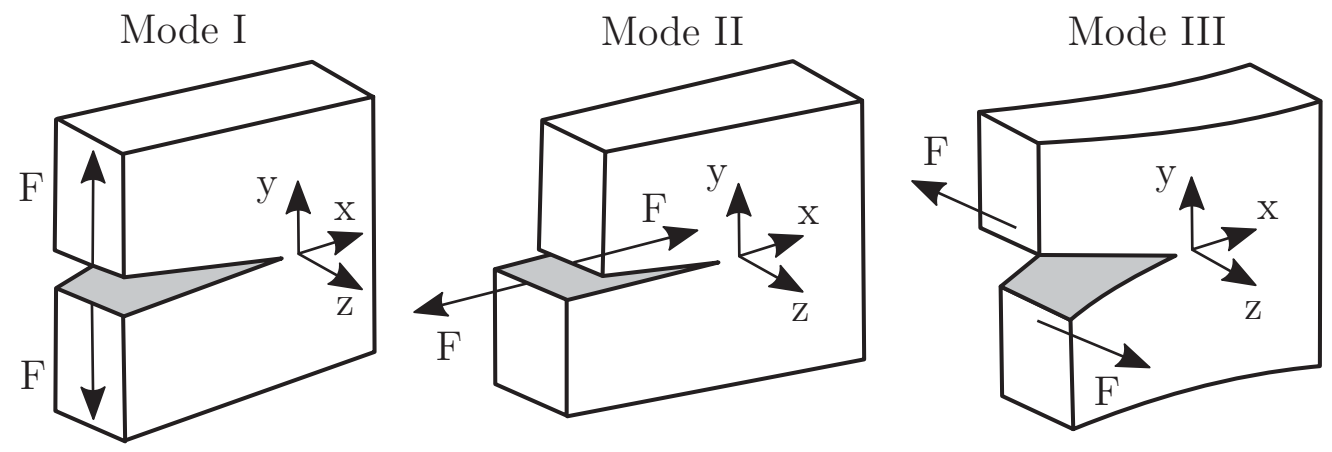

Abb. 2.13.: Grundbeanspruchungsarten eines Risses (Rissöffnungsmodi)

In der Praxis hat der Rissöffnungsmode I die größte Bedeutung. Er tritt vorrangig bei Bauteilen mit Innen-, Oberflächen- oder Durchrissen auf, die auf Zug, Biegung oder Innendruck beansprucht werden. Er ist auch für die in dieser Arbeit untersuchten gelochten Bauteile aus alten Stählen maßgebend. Da die Bruchmodi II und III bei der Lösung der vorliegenden Aufgabenstellung keine Bedeutung haben, werden sie im Folgenden nicht weiter betrachtet.

\section{Linear-Elastische Bruchmechanik (LEBM)}

Das Konzept der linear-elastischen Bruchmechanik beschreibt das Versagen eines Bauteils mit Riss als Folge einer instabilen Rissausbreitung. Voraussetzung dafür ist ein weitgehend linear-elastisches Werkstoffverhalten bis zum Bruch. Die Spannungen und Verschiebungen vor der Rissspitze eines Innenrisses, in einer unendlichen, homogenen, isotropen und gleichmäßig auf Zug beanspruchten Scheibe (siehe Abbildung 2.14), werden mit den IrwinWilliams-Gleichungen in Abhängigkeit des Abstandes $r$ zur Rissspitze beschrieben.

$$
\begin{aligned}
\left(\begin{array}{c}
\sigma_{x} \\
\sigma_{y} \\
\tau_{x y}
\end{array}\right) & =\frac{K_{I}}{\sqrt{2 \pi r}} \cdot \cos \frac{\phi}{2} \cdot\left(\begin{array}{c}
1-\sin \frac{1}{2} \phi \cdot \sin \frac{3}{2} \phi \\
1+\sin \frac{1}{2} \phi \cdot \sin \frac{3}{2} \phi \\
\sin \frac{1}{2} \phi \cdot \cos \frac{3}{2} \phi
\end{array}\right) \\
\left(\begin{array}{c}
u \\
v
\end{array}\right) & =\frac{K_{I}}{2 E} \cdot \sqrt{\frac{r}{2 \pi}} \cdot\left(\begin{array}{c}
(1+\nu) \cdot\left[(2 \eta-1) \cdot \cos \frac{1}{2} \phi-\cos \frac{3}{2} \phi\right. \\
(1+\nu) \cdot\left[(2 \eta+1) \cdot \sin \frac{1}{2} \phi-\sin \frac{3}{2} \phi\right]
\end{array}\right)
\end{aligned}
$$



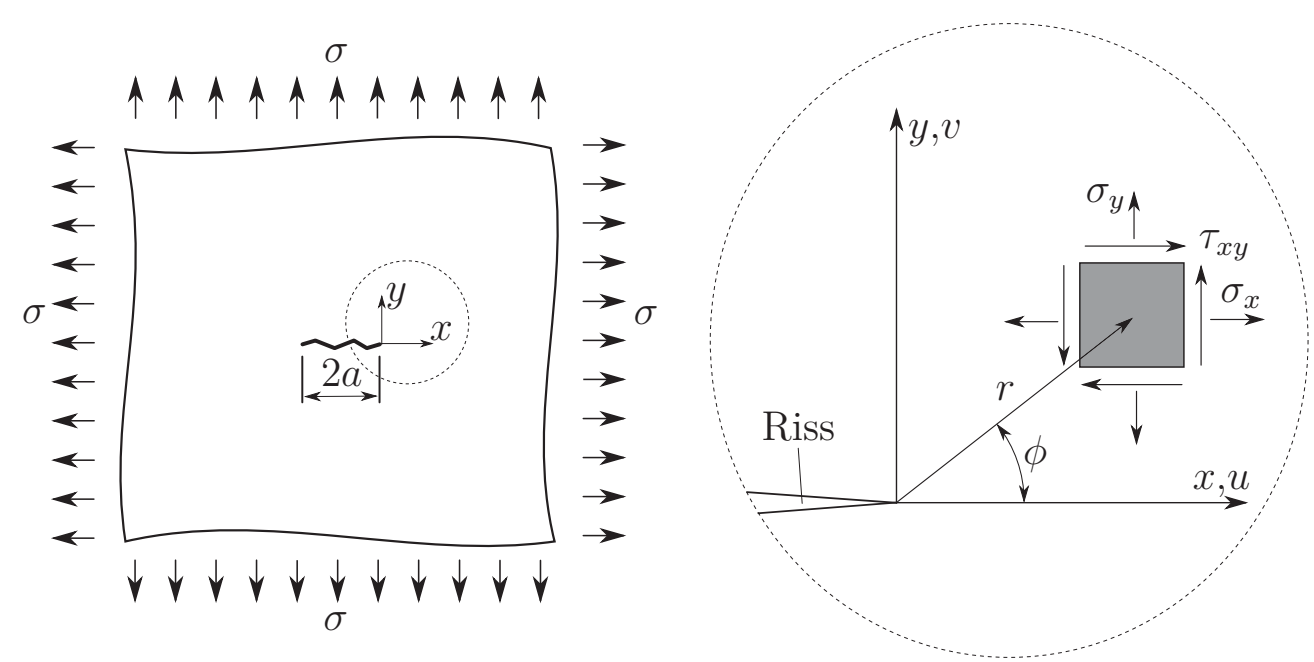

Abb. 2.14.: Unendliche Scheibe mit Innenriss, Spannungskomponenten an der Risspitze

Demnach wachsen die Spannungen mit Annäherung an die Rissspitze proportional zu 1/ $\sqrt{r}$ an und werden für $r \rightarrow 0$ unendlich groß. Diese Singularität gilt allerdings nur bei idealelastischem Werkstoffverhalten. Bei realen technischen Werkstoffen führt die Spannungskonzentration an der Rissspitze zu irreversiblen Verformungen. Als Bedingung für die Anwendbarkeit der LEBM gilt daher, dass diese Bereiche (plastische Zonen) gegenüber den Bauteil- und Rissabmessungen klein bleiben müssen. Die LEBM findet deshalb bevorzugt bei hochfesten bzw. spröden Werkstoffen ihre Anwendung.

Die Beanspruchung und das Bruchverhalten des rissbehafteten Bauteils sind abhängig von der Art des Spannungszustandes vor der Rissspitze. Bei einem ebenen Spannungszustand (ESZ), wie er bei dünnen Scheiben vorliegt, sind nur die drei Spannungskomponenten wie in Gleichung 2.1 vorhanden. Die Dehnung in Dickenrichtung lässt sich mit

$$
-\varepsilon_{z}=\nu \cdot\left(\frac{\sigma_{x}}{E}\right)+\nu \cdot\left(\frac{\sigma_{y}}{E}\right)
$$

ermitteln. Bei sehr dicken Scheiben kann infolge der Dehnungsbehinderung in Dickenrichtung die Deformation in z-Richtung vernachlässigt werden und es liegt näherungsweise ein ebener Dehnungszustand (EDZ) vor. Die zugehörige Normalspannungskomponente ergibt sich zu:

$$
\sigma_{z}=\nu \cdot\left(\sigma_{x}+\sigma_{y}\right)
$$

Der Beginn der instabilen Rissausbreitung beim Erreichen eines Grenzwertes der Spannungsintensität $K_{I c}$ (Bruch- bzw. Risszähigkeit) als Bruchkriterium

$$
K_{I} \geq K_{I c}
$$

der LEBM ist unter der Bedingung eines ebenen Dehnungszustandes an der Rissspitze definiert. Dieser EDZ wird aber nur bei sehr dicken Bauteilen bzw. Proben erreicht. Folglich ist der kritische Spannungsintensitätsfaktor eines Bauteils dickenabhängig, während $K_{I c}$ den unteren Grenzwert der Bruchzähigkeit eines Werkstoffes angibt [BlumenAuER; Pusch (1993)].

Der Spannungsintensitätsfaktor an einem Mittelriss der Länge $2 a$ in einer unendlich ausgedehnten Scheibe unter einachsiger Zugbeanspruchung $\sigma$ (im ungerissenen Bruttoquerschnitt) 
ergibt sich zu:

$$
K_{I}=\sigma \cdot \sqrt{\pi \cdot a}
$$

Die Erweiterung der Gleichung 2.6 auf endliche Geometrien ist mit Hilfe eines zusätzlichen Geometriefaktors F möglich.

$$
K_{I}=\sigma \cdot \sqrt{\pi \cdot a} \cdot F
$$

Dieser dimensionslose Faktor ist üblicherweise eine Funktion, die die Bauteil- und Rissgeometrie wie auch die konkrete Belastungssituation erfasst. Für eine Vielzahl von Geometrien und Beanspruchungen können diese Faktoren aus Handbüchern, wie z. B. MuraKAmi (1990), Wu; Carlsson (1991), Al Laham; Ainsworth et al. (1998) oder Tada; Paris et al. (2000), entnommen werden. Geeignete Handbuchlösungen für die hier zu untersuchenden gelochten Bauteile und Anschlüsse sind in Abschnitt 2.6.2 zusammengestellt.

\section{LEBM mit Kleinbereichsfließen}

Die Annahme eines idealen linear-elastischen Werkstoffverhaltens in der LEBM gilt, wie bereits oben ausgeführt, nicht für übliche technische Werkstoffe. Die Spannungsüberhöhung vor der Rissspitze führt auch bei makroskopisch sprödem Werkstoffverhalten zu irreversiblen Verformungen, d. h. zur Ausbildung einer plastischen Zone. Unter der Voraussetzung, dass diese Zone klein bleibt im Verhältnis zu den Bauteilabmessungen und dem Ligament, kann man diese durch den Ansatz einer effektiven Risslänge

$$
a_{e f f}=a+r_{p l}
$$

berücksichtigen. Die Ausdehnung $r_{p l}$ der plastischen Zone im Ligament kann grob abgeschätzt werden, in dem in Gleichung 2.1 bei $\phi=0$ für $\sigma_{y}$ die Streckgrenze $R_{e}$ des Materials eingesetzt wird:

$$
\begin{aligned}
& r_{p l}=\frac{1}{2 \cdot \pi} \cdot\left(\frac{K_{I}}{R_{e}}\right)^{2} \quad \text { für den } \mathrm{ESZ} \\
& r_{p l}=\frac{1}{2 \cdot \pi} \cdot\left(\frac{K_{I}}{R_{e}}\right)^{2} \cdot(1-\nu)^{2} \quad \text { für den } \mathrm{EDZ}
\end{aligned}
$$

Mit der Einführung der effektiven Risslänge wird der Gültigkeitsbereich der LEBM auf das Kleinbereichsfließen (small scale yielding) erweitert. Nach Blumenauer; Pusch (1993) ist eine maximale Größe der plastischen Zone $r_{p l}=0,025 a$ zulässig. Kann diese Bedingung wie bei den hier zu untersuchenden alten Flussstählen nicht eingehalten werden, müssen die Methoden der elastisch-plastischen Bruchmechanik angewendet werden.

\section{Elastisch-Plastische Bruchmechanik (EPBM)}

Die wesentlichen Konzepte der elastisch-plastischen Bruchmechanik sind das J-Integral- und das CTOD-Konzept. Das von RICE (1968) eingeführte J-Integral beschreibt den Energieumsatz an der Rissspitze. Es ist als wegunabhängiges, entgegen dem Uhrzeigersinn verlaufendes Linienintegral mit geschlossenem Integrationsweg von einem Rissufer zum anderen definiert (siehe Abbildung 2.15). Das J-Integral setzt sich aus der auf das Volumen bezogenen Formänderungsarbeit $W_{e}$ (Formänderungsdichte) und der Arbeit der äußeren Kräfte $\sigma \cdot \partial u$ in einem ebenem Verformungsfeld zusammen. 


$$
J=\int_{\Gamma} W_{e} \mathrm{~d} y-\vec{\sigma} \frac{\partial \vec{u}}{\partial x} \mathrm{~d} s
$$

mit: $W_{e}=\int_{0}^{\varepsilon} \sigma_{i j} \mathrm{~d} \varepsilon_{i j}$

$x$ : Ausbreitungsrichtung des Risses

$y$ : Richtung senkrecht zu den Rissufern

$\Gamma$ : Integrationsweg um die Rissspitze vom unteren zum oberen Rissufer

$\mathrm{d} s$ : Linienelement des Integrationsweges $\Gamma$

$\vec{\sigma}$ : ein auf das Linienelement $\mathrm{d} s$ wirkender Spannungsvektor

$\vec{u}$ : Verschiebungsvektor des Integrationsweges $\Gamma$

$\sigma_{i j}$ : Spannungstensor in den lokalen Koordinaten i j

$\varepsilon_{i j}$ : Dehnungstensor in den lokalen Koordinaten i j

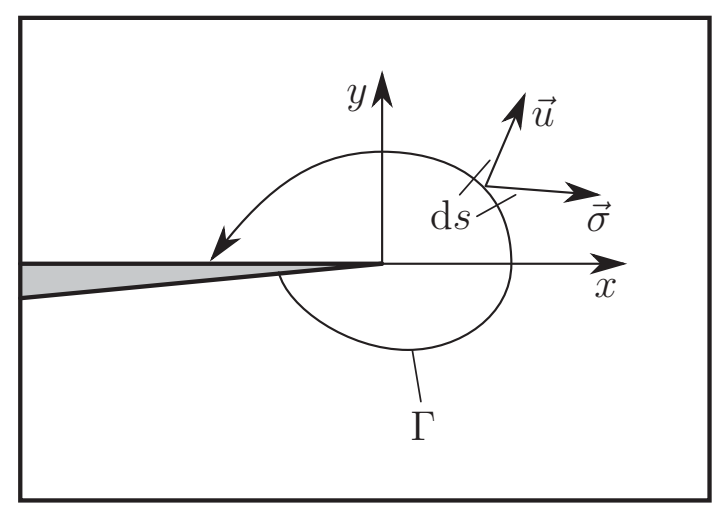

Abb. 2.15.: Definition des J-Integrals

Die experimentelle Bestimmung des J-Integrals auf Basis der Gleichung 2.11 ist sehr aufwendig, da die Dehnungen entlang des Integrationsweges mit mehreren Dehnmessstreifen gemessen werden müssten. Man macht sich daher die energetische Interpretation des J-Integrals zunutze. Demnach beschreibt es die Änderung der potentiellen Energie d $U$ vor der Rissspitze, bezogen auf die sich bildende Rissfläche d $A$ bzw. die Risslänge d $a$ bei einer Probenbreite $B$.

$$
J=-\frac{\mathrm{d} U}{\mathrm{~d} A}=-\frac{1}{B} \cdot \frac{\mathrm{d} U}{\mathrm{~d} a}
$$

Damit besitzt das J-Integral die Einheit einer Arbeit pro Fläche, die üblicherweise in N/mm angegeben wird. Unter der Voraussetzung einer monoton wachsenden Belastung ist das JIntegral in der LEBM wie auch der EPBM gültig. Im Falle linearen Werkstoffverhaltens kann es wie folgt in eine äquivalente Spannungsintensität $K_{I}$ umgerechnet werden.

$$
\begin{aligned}
& K_{I}=\sqrt{E \cdot J} \text { für den ESZ } \\
& K_{I}=\sqrt{\frac{E \cdot J}{1-\nu^{2}}} \text { für den EDZ }
\end{aligned}
$$


Das CTOD-Konzept basiert auf dem Rissmodell nach Dugdale (1960). Darin wird angenommen, dass bei duktilem Werkstoffverhalten der Bruchvorgang nicht wie beim K-Konzept durch eine kritische Spannungsintensität, sondern durch eine kritische plastische Verformung an der Rissspitze kontrolliert wird. Ein Maß für diese kritische plastische Verformung ist die Rißaufweitung $\delta$ bzw. die Rißspitzenöffnung CTOD (crack tip opening displacement).

Eine allgemeingültige Anwendung des CTOD-Konzepts wird bisher dadurch erschwert, dass die Rissöffnung unterschiedlich definiert wird. Experimentell kann diese z. B. direkt durch ein Messmikroskop an der Probenoberfläche gemessen werden. In numerischen Berechnungen mittels FEM hat sich die Definition des CTOD-Wertes $\delta_{45}$ bewährt. Dieser ermittelt sich zwischen den Schnittpunkten zweier im Winkel von $45^{\circ}$ von der Rissspitze ausgehender Geraden mit den Rissflanken. Von Schwalbe (1986) hingegen wurde eine Rissöffnung $\delta_{5}$ definiert, die von der Rissspitze aus über eine Messlänge von $5 \mathrm{~mm}$ gemessen wird. Während das CTOD-Konzept in der Praxis bei der Werkstoffwahl und der Fehlerbewertung von Schweißkonstruktionen angewendet wird [Blumenauer; Pusch (1993)], ist es für die in dieser Arbeit durchgeführten Untersuchungen genieteter Flussstahlkonstruktionen nicht von Bedeutung.

\subsubsection{Spannungsintensitätsfaktoren für Lochstäbe}

Ausgehend von dem an einer unendlich ausgedehnten Scheibe mit Innenriss abgeleiteten Spannungsintensitätsfaktor $K_{I}$ in Gleichung 2.6 wurden unterschiedliche rechnerische Ansätze abgeleitet, die die Berücksichtigung anderer Geometrien ermöglichen. Die verschiedenen Einflussgrößen, wie z. B. der Breiteneinfluss endlich breiter Bauteile oder der Einfluss verschiedener Belastungssituationen, können aufgrund der Linearität der Berechnungsansätze nach dem Superpositionsprinzip miteinander kombiniert werden. Dadurch wird es möglich, eine Vielzahl unterschiedlicher Geometrie- und Belastungskonfigurationen abzubilden. Im Folgenden werden Rechenansätze vorgestellt, die zur Untersuchung gelochter Bauteile und genieteter bzw. geschraubter Anschlüsse geeignet sind. Allen Ansätzen ist die Annahme eines Durchrisses mit gerader Rissfront zugrunde gelegt, der vom Rand eines Loches oder Bauteils ausgeht.

Mit den Untersuchungen von Hensen (1992) und den weiterführenden Arbeiten von GEISSLER (1995), LANGENBERG (1996) und StÖTzEL (1998) wurden wesentliche Grundlagen zur Bewertung der Restnutzungsdauer und Sprödbruchsicherheit genieteter Eisenbahnbrücken aus Flussstahl auf bruchmechanischer Basis erarbeitet. Dabei führten die Autoren alle wesentlichen Konstruktionsdetails vereinfachend auf die bruchmechanischen Rissmodelle CCT (center cracked tension), DECT (double edge cracked tension) und SECT (single edge cracked tension) nach TADA; PARIS et al. (2000) zurück (siehe auch Abbildung 2.16). Die zugehörigen Geometriefunktionen sind mit Gleichung 2.16 bis 2.18 angegeben. Basierend auf numerischen Vergleichsrechnungen wurden für Bauteile, mit von den Modellen abweichender Geometrie, z. B. Winkelprofile mit asymmetrischem Loch in nur einem Schenkel, äquivalente Modellbreiten $W$ bestimmt, so dass mit den o. g. Rissmodellen die Rissspitzenbeanspruchung konservativ abgeschätzt werden konnte. Diese Vereinfachung war erforderlich, da der Nachweis gegen Sprödbruch auf Basis des J-Integral-Konzepts geführt wurde und nur für diese drei Modelle J-Integral-Lösungen vorlagen. 


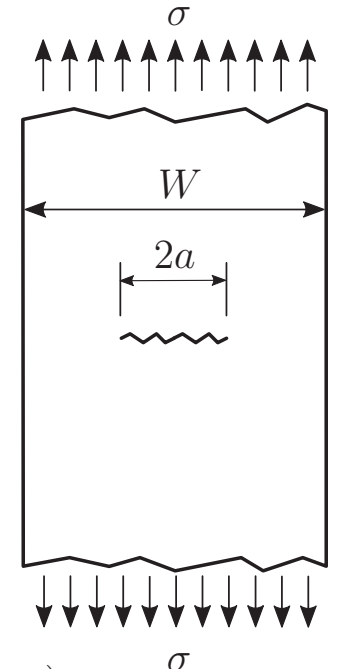

a)

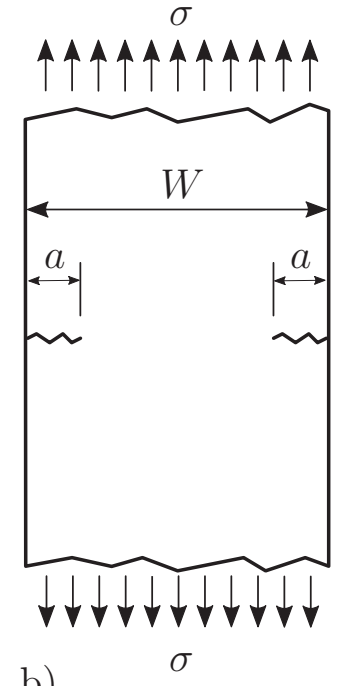

b)

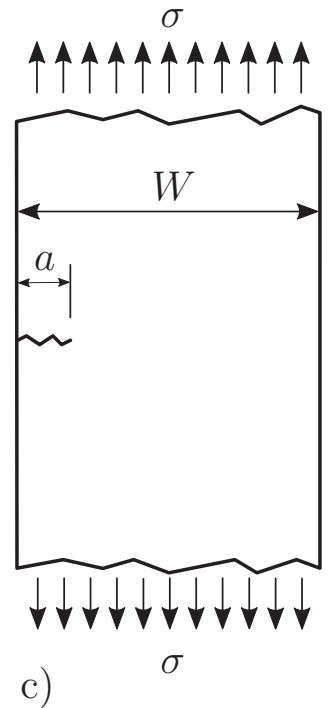

c)

Abb. 2.16.: Rissgeometrien zur Untersuchung genieteter Bauteile bei Hensen (1992), LANGENBerg (1996) und Stötzel (1998), a) Blech mit Mittenriss (CCT), b) Blech mit symmetrischem Randriss (DECT), c) Blech mit einseitigem Randriss (SECT)

$$
\begin{gathered}
K_{I}=\sigma \cdot \sqrt{\pi \cdot a} \cdot F\left(\frac{a}{W}\right) \\
F_{C C T}=\left[1-0,025 \cdot\left(\frac{2 a}{W}\right)+0,06 \cdot\left(\frac{2 a}{W}\right)^{2}\right] \cdot \sqrt{\sec \left(\frac{\pi a}{W}\right)} \\
F_{D E C T}=\frac{1,122-0,561 \cdot\left(\frac{2 a}{W}\right)-0,205 \cdot\left(\frac{2 a}{W}\right)^{2}+0,471 \cdot\left(\frac{2 a}{W}\right)^{3}-0,19 \cdot\left(\frac{2 a}{W}\right)^{4}}{\sqrt{1-\left(\frac{2 a}{W}\right)}} \\
F_{S E C T}=\left[\frac{0,752+2,02 \cdot\left(\frac{a}{W}\right)+0,37 \cdot\left(1-\sin \left(\frac{\pi a}{2 W}\right)\right)^{3}}{\cos \left(\frac{\pi a}{2 W}\right)}\right] \cdot \sqrt{\frac{2 W}{\pi a} \cdot \tan \left(\frac{\pi a}{2 W}\right)}
\end{gathered}
$$

Alle drei o. g. Modelle beschreiben die Rissspitzenbeanspruchung an einem Riss, der in genieteten Konstruktionen üblicherweise von einem Nietlochrand ausgeht, berücksichtigen aber die Kerbwirkung durch die Löcher selbst nicht. Während der Einfluss der Löcher in den kräftigen Querschnitten genieteter Stahlbrückenkonstruktionen relativ gering ist, darf er bei der Bewertung schlanker Bauteile im Stahlhochbau nicht vernachlässigt werden. Hier entspricht der Nietlochdurchmesser oft bis zu einem Drittel der Profilbreite (siehe Tabelle 2.5 und 2.6). Durch Vernachlässigung der Kerbwirkung der Löcher wird die Rissspitzenbeanspruchung am symmetrischen Innenriss mit dem Modell CCT um bis zu 15\% unterschätzt. Dies wird bei Gegenüberstellung mit dem Modell eines Blechstreifens mit symmetrischem Mittenriss am Loch nach NEWMAN (1971) in Abbildung 2.17 deutlich. Die zugehörige Formfunktion enthält Gleichung 2.19. 

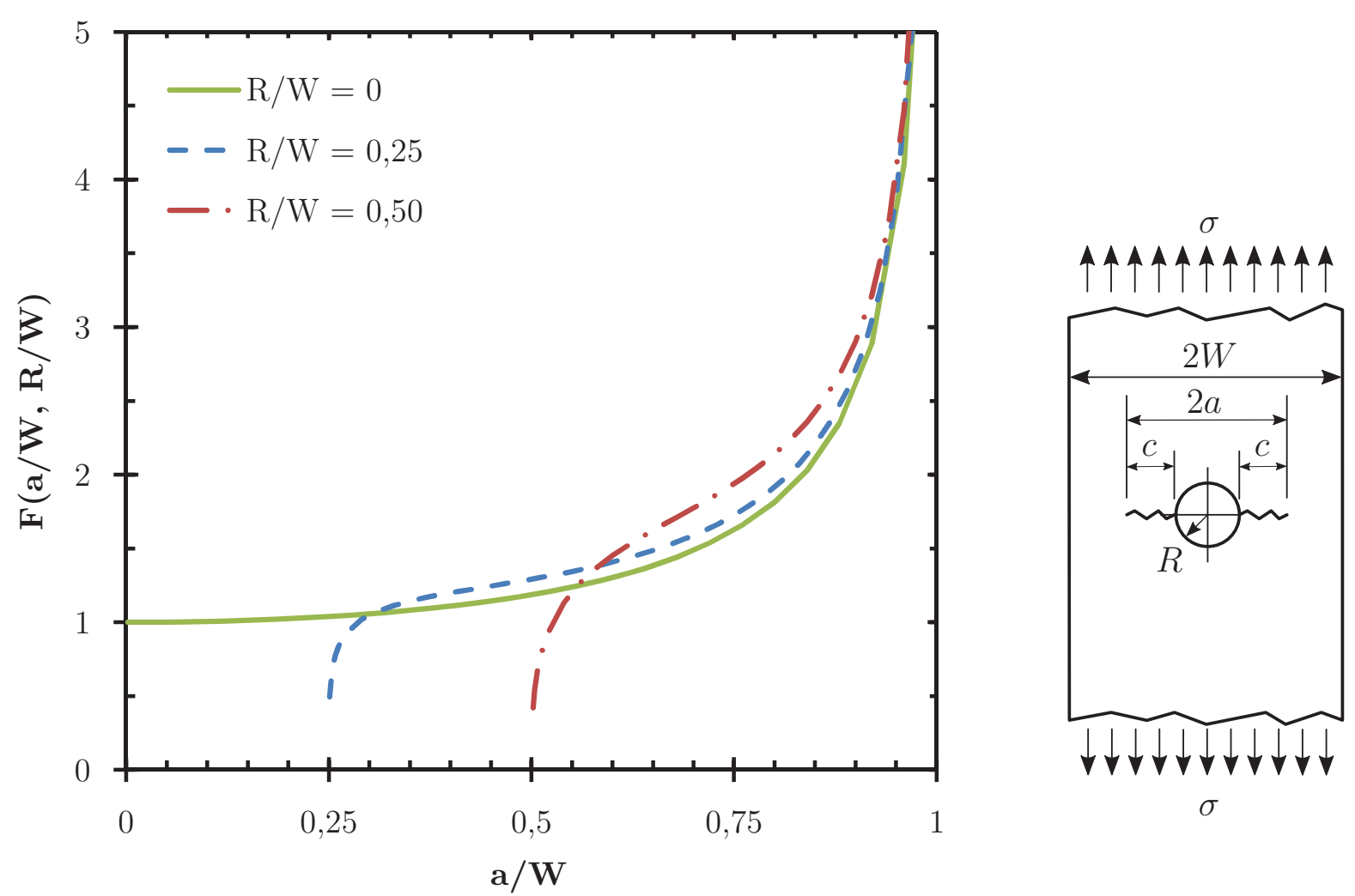

Abb. 2.17.: Einfluss des Lochdurchmessers bei der Berechnung eines Blechs mit Loch und beidseitig symmetrischem Durchriss nach Newman (1971)

$$
F=\frac{\pi\left[\sqrt{\frac{2 W}{\pi a}\left(\tan \frac{\pi a}{2 W}+0,0325 \cdot \sin \frac{\pi a}{W}\right)} \cdot\left(1+\frac{\varepsilon^{2} \cdot\left(2-\varepsilon^{2}\right)}{1-\varepsilon}\right)\right]-1,032}{\pi-1} \cdot \psi
$$

mit: $\varepsilon=0,344 \cdot \frac{a}{W}$

$$
\begin{aligned}
\psi & =4,877 \cdot \beta^{\frac{2}{3} P}-4,1455 \cdot \beta^{P} \\
\beta & =\frac{a-R}{W-R} \\
P & =\frac{\log 0,48245}{\log \frac{R}{R+W}}
\end{aligned}
$$

Zur bruchmechanischen Untersuchung eines endlich breiten Lochstabes mit einseitigem Durchriss am Loch oder beidseitigem Riss bei exzentrischer Lage des Loches, kombiniert BALL (1987) die Lösung einer unendlich ausgedehnten Lochscheibe mit einseitigem Durchriss und die eines endlich breiten Stabes mit exzentrischem Innenriss gemäß Abbildung 2.18.

Basierend auf Gleichung 2.7 ergibt sich für diesen Fall die Rissspitzenbeanspruchung infolge 
der Zugbeanspruchung $\sigma$ im Bruttoquerschnitt (Remote Stress) zu:

$$
K_{R}=\sigma \cdot \sqrt{\pi \cdot a} \cdot F_{T H} \cdot F_{F F}
$$

Der Geometrieeinfluss des Lochs mit Durchriss (Through crack at a Hole) in der unendlich ausgedehnten Scheibe wird im Fall eines einseitigen Risses mit Gleichung 2.21 und für den beidseitig symmetrischen Riss mit Gleichung 2.22 berücksichtigt.

$$
\begin{gathered}
F_{T H}=e^{\left[1,2133-2,205(a /(a+R))+0,645(a /(a+R))^{2}\right]} \\
F_{T H}=\frac{0,6805}{0,2772+a / R}+0,9439
\end{gathered}
$$

Den Einfluss der endlichen Breite des Stabes (Finite Width) beschreibt jeweils der erste Term in den Gleichungen 2.23 und 2.24. Die zweiten Terme berücksichtigen den Einfluss der Exzentrizität (Eccentricity) des Risses. Gleichung 2.23 bezieht sich dabei auf die näher zur Bauteilkante gelegene Rissspitze A und Gleichung 2.24 auf die gegenüberliegende, weiter vom Rand entfernte Rissspitze B.

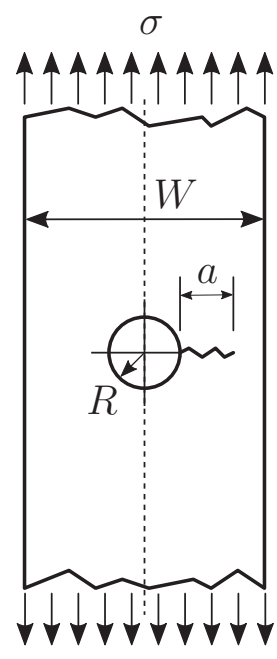

$\sigma$

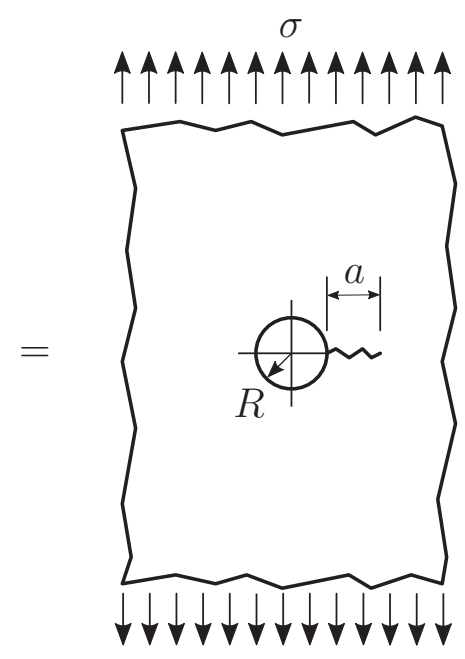

$\sigma$

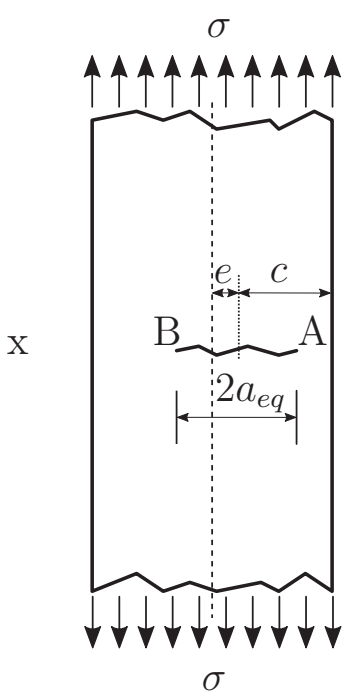

$\sigma$

Abb. 2.18.: Berechnung des einseitig angerissenen Lochstabes als Kombination einer unendlich ausgedehnten, einseitig angerissenen Lochscheibe und eines endlich breiten Stabes mit exzentrischem Innenriss nach BALL (1987)

mit: $\sigma$ : Zugspannung im Bruttoquerschnitt des Stabes

$a_{e q}$ : Halbe equivalente Risslänge

$c$ : Abstand der Riss-Mittellinie zur nähergelegenen Stabkante

$e:$ Abstand zwischen Riss-Mittellinie und Stab-Mittelllinie (Rissexzentrizität)

$W$ : Stabbreite

$A, B$ : Bezeichnung der Rissspitze 


$$
\begin{aligned}
F_{F F} & =F_{F W}+F_{E C C} \\
F_{F F, A} & =\sqrt{\sec \left[\frac{\pi a_{e q}}{2 c}\right]}-0,6173\left(\frac{a_{e q}}{c}\right)^{2,6} \cdot\left(\frac{2 e}{W}\right)^{2} \\
F_{F F, B} & =\sqrt{\sec \left[\frac{\pi a_{e q}}{2 c}\right]}-1,0522\left(\frac{a_{e q}}{c}\right)^{2,9} \cdot\left(\frac{2 e}{W}\right)^{2}
\end{aligned}
$$

Allen fünf bisher beschriebenen Modellen gemein ist die Voraussetzung einer konstanten Zugbeanspruchung im gesamten betrachteten Bauteilabschnitt ohne Lasteinleitung am angerissenen Loch oder am Riss selbst. Diese Annahme gilt in genieteten oder geschraubten Stahlkonstruktionen nur außerhalb der Bauteilanschlüsse. Spannungsintensitätsfaktoren für Risse an Löchern in Anschlüssen wurden erstmalig von NEWMAN (1971) abgeschätzt (siehe Abbildung 2.19).
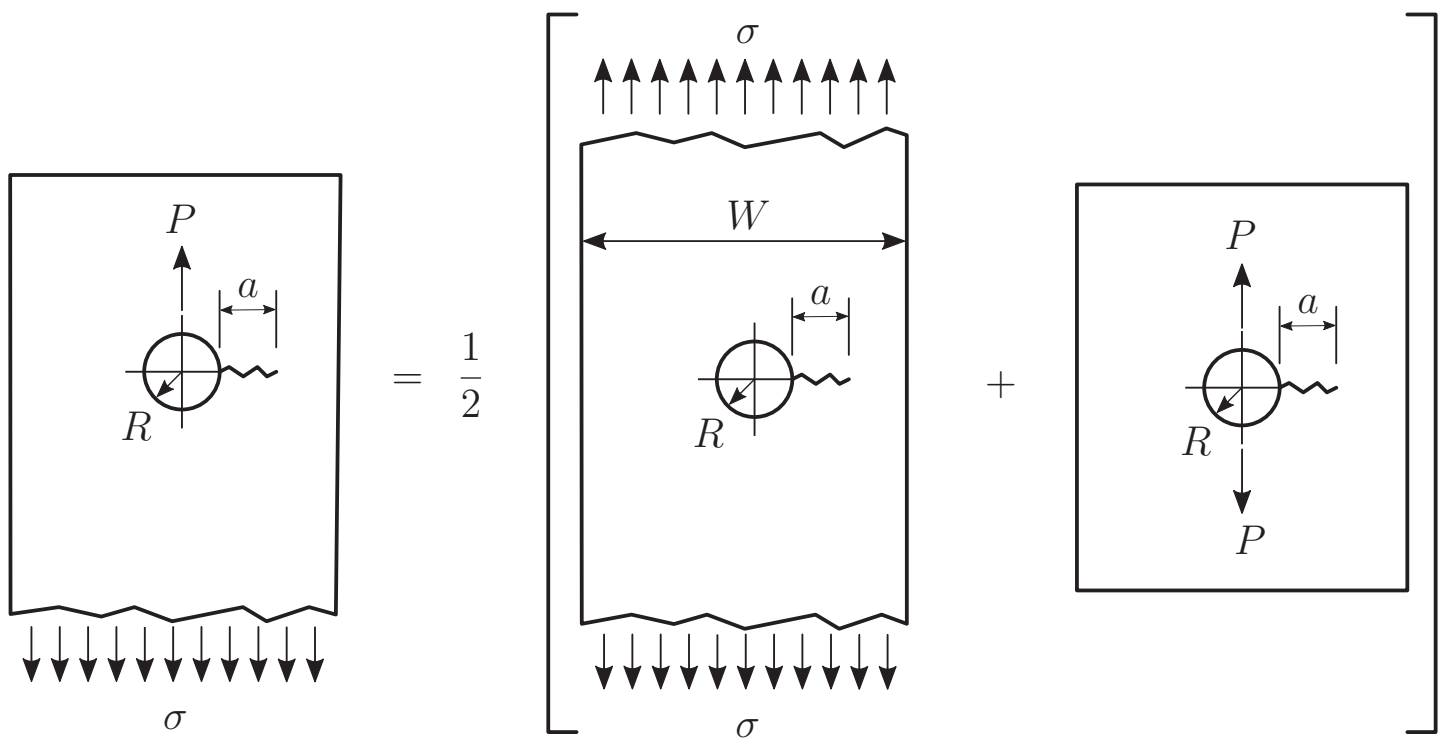

Abb. 2.19.: Superposition nach NeWMAn (1971)

Aufbauend auf diesem Superpositionsprinzip beschreibt BALL (1987) die Beanspruchung $K_{I}$ am Riss als Kombination der Spannungsintensität $K_{R}$ nach Gleichung 2.20 infolge der Zugbeanspruchung im Bauteil (Remote load) und der Spannungsintensität $K_{P}$ aus der Einleitung der Einzellast $P$ am Loch (Point load):

$$
K_{I}=\frac{1}{2}\left(K_{R}+K_{P}\right)
$$

Die Spannungsintensität infolge Einleitung einer Einzellast am Loch (siehe Abbildung 2.20) definiert BALL (1987) zu:

$$
K_{P}=\frac{P}{t \sqrt{2 c}} \cdot F_{P} \cdot F_{H P W}+\frac{P}{W t} \sqrt{\pi \cdot a} \cdot F_{T H} \cdot F_{E C C}
$$


Die Berücksichtigung des Durchrisses am exzentrischen Loch erfolgt auch hier durch die beiden Geometriefaktoren $F_{T H}$ und $F_{E C C}$ (Gleichungen 2.21 bis 2.24). Der Einfluss der Lasteinleitung am Lochrand auf die Rissspitzenbeanspruchung wird mit dem Geometriefaktor $F_{P}$ erfasst, der wie folgt berechnet wird:

$$
\begin{gathered}
F_{P}=\left\{1+0,2967 \sqrt{1-\left(g / a_{e q}\right)^{2}}\left[1-\cos \left(\frac{\pi a_{e q}}{2 c}\right)\right]\right\} \cdot F_{I I I} \\
F_{I I I}=\frac{\sqrt{\tan \left(\frac{\pi a_{e q}}{2 c}\right)}\left\{1-\left[\sin \left(\frac{\pi g}{2 c}\right) / \sin \left(\frac{\pi a_{e q}}{2 c}\right)\right]\right\}}{\sqrt{1-\left[\cos \left(\frac{\pi a_{e q}}{2 c}\right) / \cos \left(\frac{\pi g}{2 c}\right)\right]^{2}}}
\end{gathered}
$$

Darüber hinaus beeinflusst der Abstand $h$ des Loches vom Bauteilende die Beanspruchung an der Rissspitze. Von BALL (1987) wurde dafür der Faktor $F_{H P W}$ abgeleitet, der neben der endlichen Höhe (finite Height) auch die endliche Breite des Bleches (finite Width) bei Einleitung einer Einzellast (Point load) berücksichtigt. Aus diesem Grund ist in Gleichung 2.26 der Faktor $F_{F W}$ nicht enthalten. Der Faktor $F_{H P W}$ wird mit Hilfe des Diagramms in Abbildung 2.21 bestimmt.

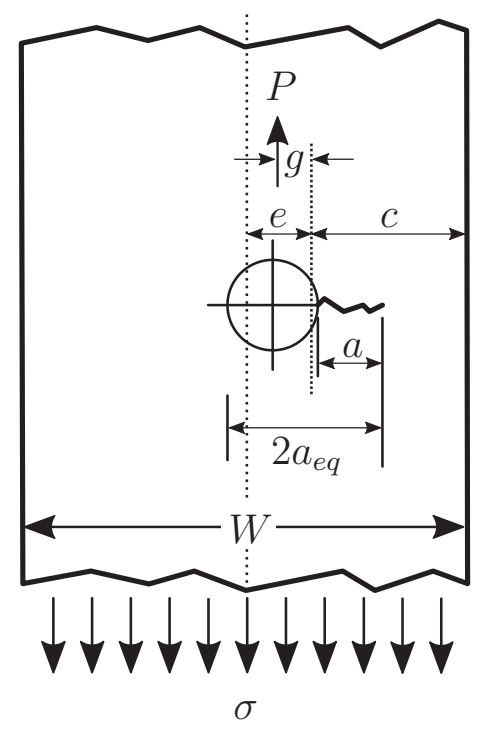

Abb. 2.20.: Geometriedefinition einer Schrauben- bzw. Nietverbindung nach BALL (1987)

mit: $\sigma:$ Zugspannung, resultierend aus der Einzellast

$P$ : Einzellast

$a$ : Vorhandene Risslänge

$a_{e q}$ : Halbe equivalente Risslänge

$c$ : Abstand von der Riss-Mittellinie zur nächstgelegenen Stabkante

$e$ : Abstand zwischen Riss-Mittellinie und Stab-Mittelllinie (Rissexzentrizität)

$g$ : Abstand zwischen Riss-Mittellinie und Wirkungslinie der Einzellast

$W:$ Stabbreite 


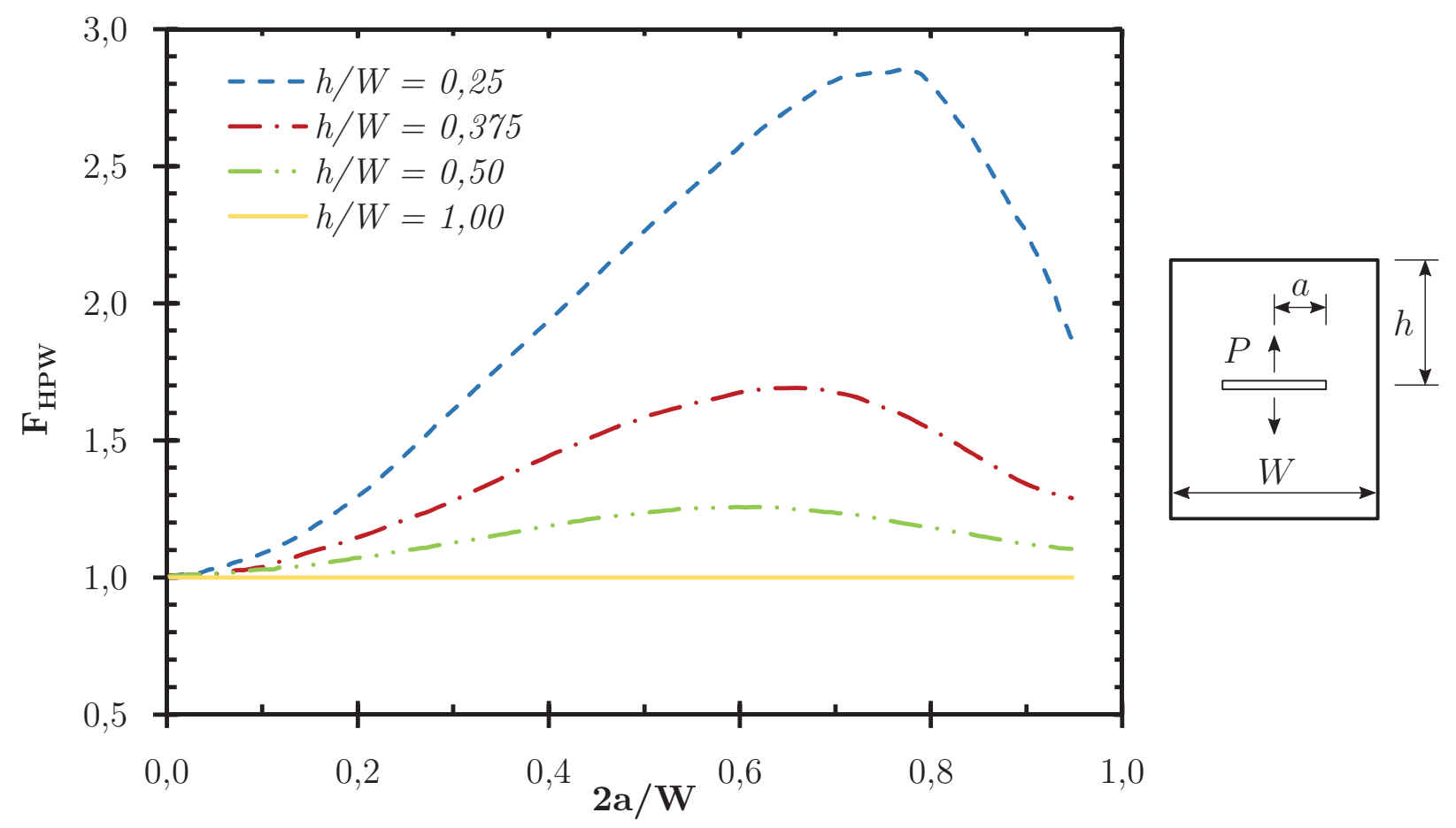

Abb. 2.21.: Höhenfaktor $F_{H P W}$ für Bleche mit endlicher Breite und Höhe nach BALL (1987)

Für den speziellen Fall einer Verbindung mit nur einem Bolzen, wie z. B. an Laschen oder Ösen, gibt Forman; Shivakumar et al. (2002) eine Lösung für den einseitigen radialen Durchriss am Lochrand an (siehe Abbildung 2.22).

$$
K_{I, \text { Verbindung }}=\frac{P}{D t} \cdot \sqrt{\pi \cdot a} \cdot F_{T C H}\left(\frac{a}{D}, \frac{D}{W}\right)
$$

Der Korrekturfaktor $F_{T C H}$ beschreibt dabei die Geometrie der Lasche wie die des Durchrisses an der Lochkante (Through Crack at a Hole).

$$
F_{T C H}=\left(\frac{D}{2 W} \cdot G_{0}+G_{1}\right) \cdot G_{W} \cdot G_{2}
$$

Analog zur Lösung von BALL (1987) superponiert der Faktor $F_{T C H}$ die Spannungsintensität des gleichmäßig im Bruttoquerschnitt gezogenen Lochstabes (Funktion $G_{0}$ ) mit der infolge konzentrierter Lasteinleitung am Lochrand (Funktion $G_{1}$ ).

$$
\begin{aligned}
& G_{0}=0,7071+0,7548 z+0,3415 z^{2}+0,642 z^{3}+0,9196 z^{4} \\
& G_{1}=0,078 z+0,7588 z^{2}+0,4293 z^{3}+0,0644 z^{4}+0,651 z^{5}
\end{aligned}
$$

mit

$$
z=\frac{1}{1+2 a / D}
$$




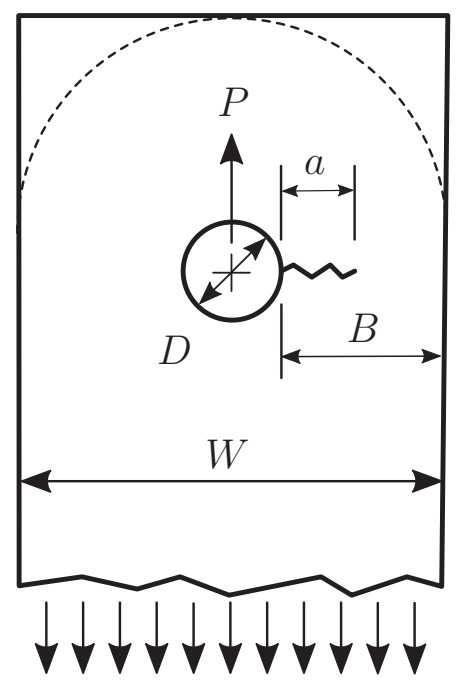

$$
\sigma=P /(W \cdot t)
$$

Abb. 2.22.: Lasche mit einseitigem Durchriss am Lochrand nach Forman; ShIVAKumar et al. (2002)

Die Laschen- und Rissgeometrie sowie die Rissexzentrizität wird durch die Funktion $G_{W}$ und den Kurvenanpassungsfaktor $G_{2}$ beschrieben.

$$
\begin{gathered}
G_{w}=\sqrt{\sec \left(\frac{\pi D}{2 W}\right) \cdot \sec \left(\frac{\pi(D+a)}{2(W-a)}\right)} \\
G_{2}=C_{1}+C_{2}\left(\frac{a}{B}\right)+C_{3}\left(\frac{a}{B}\right)^{2}+C_{4}\left(\frac{a}{B}\right)^{3}
\end{gathered}
$$

mit

$$
\begin{aligned}
& C_{1}=0,688+0,772\left(\frac{D}{W}\right)+0,613\left(\frac{D}{W}\right)^{2} \\
& C_{2}=4,984-17,318\left(\frac{D}{W}\right)+16,785\left(\frac{D}{W}\right)^{2} \\
& C_{3}=-14,297+62,994\left(\frac{D}{W}\right)-69,818\left(\frac{D}{W}\right)^{2} \\
& C_{4}=12,35-58,644\left(\frac{D}{W}\right)+66,387\left(\frac{D}{W}\right)^{2}
\end{aligned}
$$

Allen bisher beschriebenen Modellen liegt die Annahme einer konstanten Zugbeanspruchung im Bruttoquerschnitt zugrunde. Werden die Zugspannungen im Bauteil, wie bei den in Abschnitt 5.3.1 zu untersuchenden Winkelprofilen, maßgeblich durch eine Biegebeanspruchung beeinflusst, müssen diese bei der Ermittlung der Spannungsintensität berücksichtigt werden. Hierfür haben sich Lösungen mit Gewichtsfunktionen bewehrt. Von Wu; CaRLsson (1991) wurde u. a. eine solche Lösung für die endlich breite Scheibe mit mittigem Loch und einseitigem Durchriss unter ungleichmäßiger Zugbeanspruchung (siehe Abbildung 2.23) hergeleitet.

Betrachtet wird das gerissene Ligament mit der Breite $W=B-R$ mit der Koordinate $X$ entlang des Risses und dem Koordinatenursprung am Lochrand am Punkt der Rissinitiierung. 

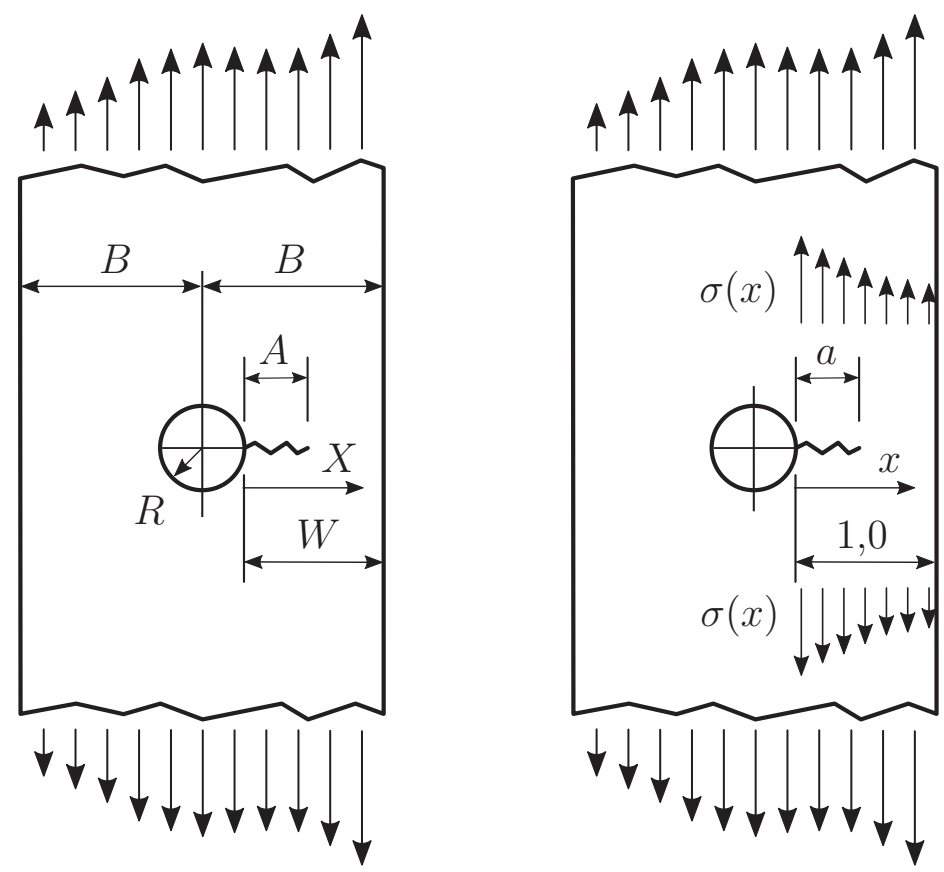

Abb. 2.23.: Geometriedefinition eines Blechstreifens mit mittigem Loch und einseitigem radialen Durchriss unter ungleichmäßiger Zugbeanspruchung nach Wu; CARLsson (1991)

Normiert auf die Ligamentbreite $W$ ergeben sich folgende dimensionslose Größen:

$$
a=\frac{A}{W}, \quad x=\frac{X}{W} \quad \text { mit } 0 \leq x \leq 1,0
$$

Die ungleichmäßige Zugbeanspruchung $\sigma(x)$ im Ligament des ungerissenen Querschnitts, die beispielsweise mit einer FEM-Analyse ermittelt werden kann, wird mit einem Polynom max. 6. Grades in folgender Form approximiert. Entscheidend ist dabei, dass die Approximation die Spannungen entlang des Risses möglichst genau beschreibt.

$$
\frac{\sigma(x)}{\sigma}=\sum_{n=0}^{N} S_{n} \cdot x^{n} \quad \text { mit } N \leq 6 \quad \text { für } 0 \leq x \leq a
$$

Die Spannungsintensität ergibt sich dann nach folgender Gleichung:

$$
K_{I}=f \cdot \sigma \cdot \sqrt{\pi \cdot a \cdot W}
$$

Der normierte Spannungsintensitätsfaktor $f$ für die entsprechende Belastungssituation wird berechnet als Superposition der Basislösungen $f_{n}$ multipliziert mit den Koeffizienten $S_{n}$ des Spannungspolynoms in folgender Form:

$$
f=\sum_{n=0}^{N} S_{n} \cdot f_{n}
$$

Die Basislösungen des Spannungsintensitätsfaktors $f_{n}$ nach Wu; CARLSSON (1991) sind in Abhängigkeit der Modell- und Rissgeometrie in Tabelle 2.8 zusammengestellt. 
Tab. 2.8.: Spannungsintensitätsfaktor $f_{n}$ für einen Blechstreifen mit Loch und einseitigem radialem Durchriss unter einer Zugspannung mit dem Potenzansatz $\sigma(x) / \sigma=x^{n}$

\begin{tabular}{|c|c|c|c|c|c|c|c|}
\hline \multicolumn{4}{|c|}{$B / R=2,0$} & \multicolumn{2}{|l|}{$n$} & \multirow[b]{2}{*}{5} & \multirow[b]{2}{*}{6} \\
\hline$a$ & 0 & 1 & 2 & 3 & 4 & & \\
\hline 0,01 & 1,1207 & $6,858 \mathrm{E}-03$ & $5,283 \mathrm{E}-05$ & $4,433 \mathrm{E}-07$ & $3,888 \mathrm{E}-09$ & $3,501 \mathrm{E}-11$ & $3,210 \mathrm{E}-13$ \\
\hline 0,05 & 1,1128 & $3,410 \mathrm{E}-02$ & $1,315 \mathrm{E}-03$ & $5,520 \mathrm{E}-05$ & $2,422 \mathrm{E}-06$ & $1,091 \mathrm{E}-07$ & $5,002 \mathrm{E}-09$ \\
\hline 0,10 & 1,1002 & $6,768 \mathrm{E}-02$ & $5,228 \mathrm{E}-03$ & $4,396 \mathrm{E}-04$ & $3,860 \mathrm{E}-05$ & $3,479 \mathrm{E}-06$ & $3,192 \mathrm{E}-07$ \\
\hline 0,20 & 1,0887 & $1,347 \mathrm{E}-01$ & $2,085 \mathrm{E}-02$ & $3,509 \mathrm{E}-03$ & $6,167 \mathrm{E}-04$ & $1,112 \mathrm{E}-04$ & $2,041 \mathrm{E}-05$ \\
\hline 0,30 & 1,1026 & $2,040 \mathrm{E}-01$ & $4,730 \mathrm{E}-02$ & $1,193 \mathrm{E}-02$ & $3,140 \mathrm{E}-03$ & $8,489 \mathrm{E}-04$ & $2,336 \mathrm{E}-04$ \\
\hline 0,40 & 1,1354 & $2,776 \mathrm{E}-01$ & $8,539 \mathrm{E}-02$ & $2,862 \mathrm{E}-02$ & $1,003 \mathrm{E}-02$ & $3,610 \mathrm{E}-03$ & $1,323 \mathrm{E}-03$ \\
\hline 0,50 & 1,1878 & $3,583 \mathrm{E}-01$ & $1,368 \mathrm{E}-01$ & $5,705 \mathrm{E}-02$ & $2,491 \mathrm{E}-02$ & $1,118 \mathrm{E}-02$ & $5,113 \mathrm{E}-03$ \\
\hline 0,60 & 1,2701 & $4,517 \mathrm{E}-01$ & $2,048 \mathrm{E}-01$ & $1,018 \mathrm{E}-01$ & $5,308 \mathrm{E}-02$ & $2,849 \mathrm{E}-02$ & $1,559 \mathrm{E}-02$ \\
\hline 0,70 & 1,4007 & $5,675 \mathrm{E}-01$ & $2,958 \mathrm{E}-01$ & $1,699 \mathrm{E}-01$ & $1,026 \mathrm{E}-01$ & $6,393 \mathrm{E}-02$ & $4,065 \mathrm{E}-02$ \\
\hline \multicolumn{8}{|c|}{$B / R=2,5$} \\
\hline 0,01 & 1,1141 & $6,832 \mathrm{E}-03$ & $5,268 \mathrm{E}-05$ & $4,423 \mathrm{E}-07$ & $3,880 \mathrm{E}-09$ & $3,495 \mathrm{E}-11$ & $3,205 \mathrm{E}-13$ \\
\hline 0,05 & 1,0850 & $3,354 \mathrm{E}-02$ & $1,299 \mathrm{E}-03$ & $5,467 \mathrm{E}-05$ & $2,402 \mathrm{E}-06$ & $1,083 \mathrm{E}-07$ & $4,972 \mathrm{E}-09$ \\
\hline 0,10 & 1,0565 & $6,595 \mathrm{E}-02$ & $5,130 \mathrm{E}-03$ & $4,331 \mathrm{E}-04$ & $3,812 \mathrm{E}-05$ & $3,443 \mathrm{E}-06$ & $3,163 \mathrm{E}-07$ \\
\hline 0,20 & 1,0300 & $1,301 \mathrm{E}-01$ & $2,034 \mathrm{E}-02$ & $3,442 \mathrm{E}-03$ & $6,069 \mathrm{E}-04$ & $1,097 \mathrm{E}-04$ & $2,017 \mathrm{E}-05$ \\
\hline 0,30 & 1,0332 & $1,960 \mathrm{E}-01$ & $4,594 \mathrm{E}-02$ & $1,166 \mathrm{E}-02$ & $3,082 \mathrm{E}-03$ & $8,356 \mathrm{E}-04$ & $2,304 \mathrm{E}-04$ \\
\hline 0,40 & 1,0544 & $2,652 \mathrm{E}-01$ & $8,263 \mathrm{E}-02$ & $2,790 \mathrm{E}-02$ & $9,821 \mathrm{E}-03$ & $3,546 \mathrm{E}-03$ & $1,303 \mathrm{E}-03$ \\
\hline 0,50 & 1,0961 & $3,413 \mathrm{E}-01$ & $1,322 \mathrm{E}-01$ & $5,555 \mathrm{E}-02$ & $2,438 \mathrm{E}-02$ & $1,098 \mathrm{E}-02$ & $5,033 \mathrm{E}-03$ \\
\hline 0,60 & 1,1679 & $4,294 \mathrm{E}-01$ & $1,976 \mathrm{E}-01$ & $9,903 \mathrm{E}-02$ & $5,191 \mathrm{E}-02$ & $2,796 \mathrm{E}-02$ & $1,534 \mathrm{E}-02$ \\
\hline 0,70 & 1,2852 & $5,385 \mathrm{E}-01$ & $2,850 \mathrm{E}-01$ & $1,651 \mathrm{E}-01$ & $1,003 \mathrm{E}-01$ & $6,268 \mathrm{E}-02$ & $3,996 \mathrm{E}-02$ \\
\hline \multicolumn{8}{|c|}{$B / R=3,5$} \\
\hline 0,01 & 1,1031 & $6,788 \mathrm{E}-03$ & $5,243 \mathrm{E}-05$ & $4,406 \mathrm{E}-07$ & $3,868 \mathrm{E}-09$ & $3,486 \mathrm{E}-11$ & $3,198 \mathrm{E}-13$ \\
\hline 0,05 & 1,0418 & $3,267 \mathrm{E}-02$ & $1,274 \mathrm{E}-03$ & $5,384 \mathrm{E}-05$ & $2,372 \mathrm{E}-06$ & $1,072 \mathrm{E}-07$ & $4,926 \mathrm{E}-09$ \\
\hline 0,10 & 0,9998 & $6,369 \mathrm{E}-02$ & $5,002 \mathrm{E}-03$ & $4,245 \mathrm{E}-04$ & $3,750 \mathrm{E}-05$ & $3,395 \mathrm{E}-06$ & $3,125 \mathrm{E}-07$ \\
\hline 0,20 & 0,9686 & $1,252 \mathrm{E}-01$ & $1,978 \mathrm{E}-02$ & $3,368 \mathrm{E}-03$ & $5,962 \mathrm{E}-04$ & $1,081 \mathrm{E}-04$ & $1,991 \mathrm{E}-05$ \\
\hline 0,30 & 0,9607 & $1,876 \mathrm{E}-01$ & $4,454 \mathrm{E}-02$ & $1,138 \mathrm{E}-02$ & $3,022 \mathrm{E}-03$ & $8,219 \mathrm{E}-04$ & $2,271 \mathrm{E}-04$ \\
\hline 0,40 & 0,9723 & $2,533 \mathrm{E}-01$ & $8,008 \mathrm{E}-02$ & $2,724 \mathrm{E}-02$ & $9,636 \mathrm{E}-03$ & $3,491 \mathrm{E}-03$ & $1,285 \mathrm{E}-03$ \\
\hline 0,50 & 1,0059 & $3,246 \mathrm{E}-01$ & $1,276 \mathrm{E}-01$ & $5,407 \mathrm{E}-02$ & $2,385 \mathrm{E}-02$ & $1,078 \mathrm{E}-02$ & $4,954 \mathrm{E}-03$ \\
\hline 0,60 & 1,0638 & $4,056 \mathrm{E}-01$ & $1,896 \mathrm{E}-01$ & $9,591 \mathrm{E}-02$ & $5,056 \mathrm{E}-02$ & $2,735 \mathrm{E}-02$ & $1,505 \mathrm{E}-02$ \\
\hline 0,70 & 1,1722 & $5,124 \mathrm{E}-01$ & $2,758 \mathrm{E}-01$ & $1,611 \mathrm{E}-01$ & $9,838 \mathrm{E}-02$ & $6,172 \mathrm{E}-02$ & $3,944 \mathrm{E}-02$ \\
\hline \multicolumn{8}{|c|}{$B / R=5,0$} \\
\hline 0,01 & 1,0936 & $6,746 \mathrm{E}-03$ & $5,218 \mathrm{E}-05$ & $4,389 \mathrm{E}-07$ & $3,855 \mathrm{E}-09$ & $3,476 \mathrm{E}-11$ & $3,190 \mathrm{E}-13$ \\
\hline 0,05 & 1,0131 & $3,210 \mathrm{E}-02$ & $1,257 \mathrm{E}-03$ & $5,329 \mathrm{E}-05$ & $2,352 \mathrm{E}-06$ & $1,064 \mathrm{E}-07$ & $4,895 \mathrm{E}-09$ \\
\hline 0,10 & 0,9590 & $6,225 \mathrm{E}-02$ & $4,926 \mathrm{E}-03$ & $4,197 \mathrm{E}-04$ & $3,717 \mathrm{E}-05$ & $3,370 \mathrm{E}-06$ & $3,105 \mathrm{E}-07$ \\
\hline 0,20 & 0,9225 & $1,221 \mathrm{E}-01$ & $1,945 \mathrm{E}-02$ & $3,326 \mathrm{E}-03$ & $5,904 \mathrm{E}-04$ & $1,072 \mathrm{E}-04$ & $1,978 \mathrm{E}-05$ \\
\hline 0,30 & 0,9072 & $1,813 \mathrm{E}-01$ & $4,347 \mathrm{E}-02$ & $1,117 \mathrm{E}-02$ & $2,976 \mathrm{E}-03$ & $8,113 \mathrm{E}-04$ & $2,246 \mathrm{E}-04$ \\
\hline 0,40 & 0,9151 & $2,451 \mathrm{E}-01$ & $7,832 \mathrm{E}-02$ & $2,680 \mathrm{E}-02$ & $9,510 \mathrm{E}-03$ & $3,453 \mathrm{E}-03$ & $1,273 \mathrm{E}-03$ \\
\hline 0,50 & 0,9468 & $3,138 \mathrm{E}-01$ & $1,246 \mathrm{E}-01$ & $5,313 \mathrm{E}-02$ & $2,351 \mathrm{E}-02$ & $1,065 \mathrm{E}-02$ & $4,904 \mathrm{E}-03$ \\
\hline 0,60 & 0,9906 & $3,862 \mathrm{E}-01$ & $1,827 \mathrm{E}-01$ & $9,303 \mathrm{E}-02$ & $4,928 \mathrm{E}-02$ & $2,675 \mathrm{E}-02$ & $1,476 \mathrm{E}-02$ \\
\hline 0,70 & 1,0851 & $4,904 \mathrm{E}-01$ & $2,675 \mathrm{E}-01$ & $1,574 \mathrm{E}-01$ & $9,654 \mathrm{E}-02$ & $6,075 \mathrm{E}-02$ & $3,891 \mathrm{E}-02$ \\
\hline
\end{tabular}




\subsubsection{Bruchmechanische Sicherheitskonzepte}

Die Bewertung der Sicherheit eines rissbehafteten Bauteils gegen Bruch erfordert im Allgemeinen den Vergleich zwischen der Rissspitzenbeanspruchung und dem im Werkstoff zur Verfügung stehenden Widerstand gegen Rissausbreitung. Wie bereits in Abschnitt 2.6.1 erläutert, treten an Rissen in typischen Baustählen plastische Verformungen auf, die die zulässige Größe der plastischen Zone nach der LEBM überschreiten. Die Berechnung der Spannungsintensität $K_{I}$ unterschätzt folglich die tatsächliche Rissspitzenbeanspruchung mit zunehmender Ligamentplastizierung (siehe Abbildung 2.24). Demnach muss die Abschätzung kritischer Beanspruchungen oder zulässiger Fehlergrößen nach den Konzepten der EPBM erfolgen, z. B. auf Basis des J-Integrals.

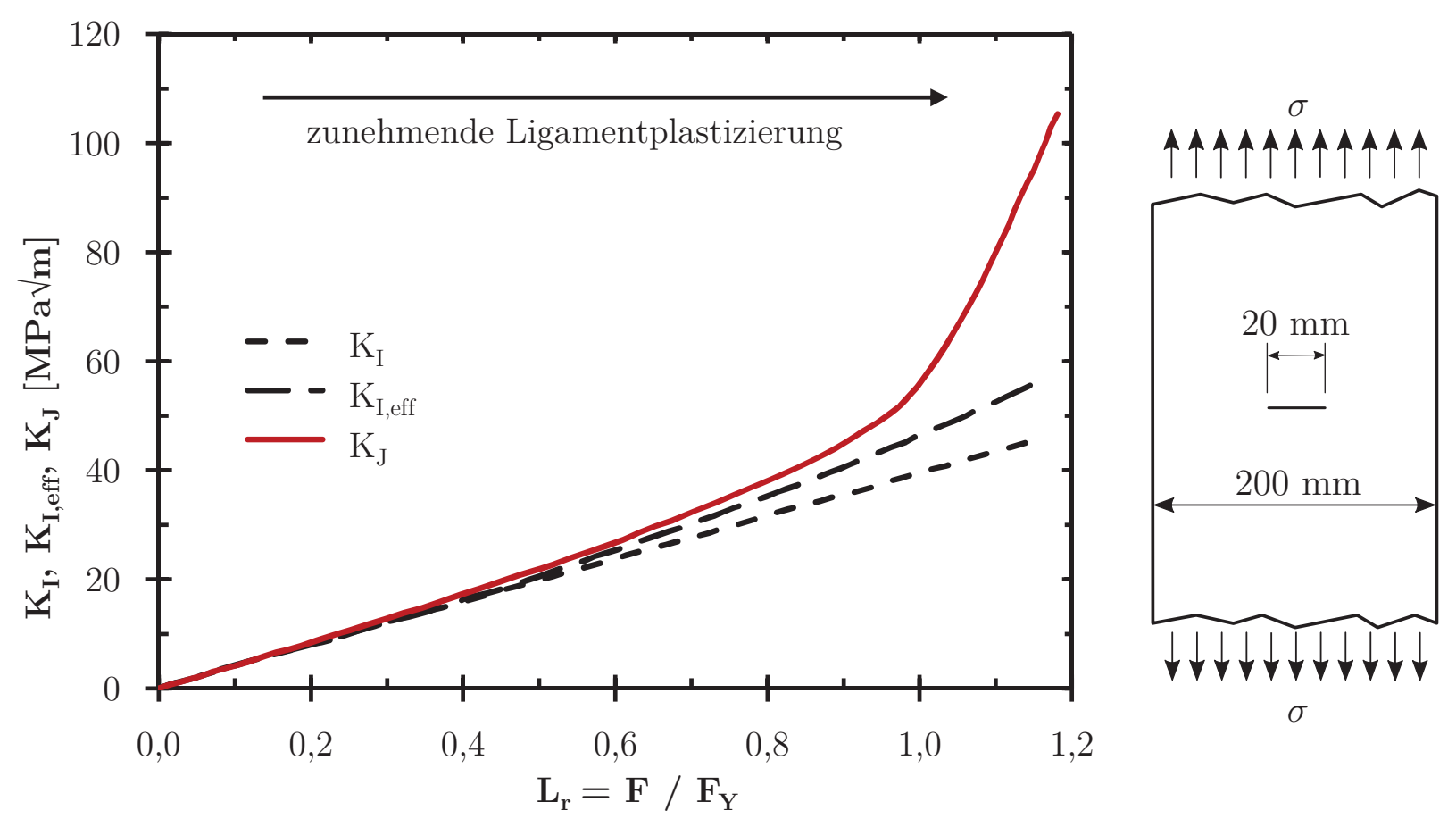

Abb. 2.24.: Rissspitzenbeanspruchung bei elastischer und elastisch-plastischer Ligamentverformung bei einem Werkstoff ohne Lüdersplateau [ZERBST; SchÖDEL et al. (2007)]

Die Berechnung des J-Integrals für komplexe Bauteile oder Risskonfigurationen erfolgt üblicherweise mittels FEM, ist aber nicht zuletzt wegen des zu berücksichtigenden nichtlinearen Werkstoffverhaltens sehr aufwendig. Für gebräuchliche Bauteil- und Risskonfigurationen wurde das J-Integral deshalb durch Kumar; GERMAn et al. (1981) in 2D-FEMBerechnungen, unter Ansatz elastisch-plastischen Werkstoffverhaltens, ermittelt und tabellarisch im EPRI-Handbuch zur Verfügung gestellt. Der Nachweis erfolgte nach der EPRIMethode im CDF-Diagramm (crack driving force).

Die Beanspruchungsgröße $J$ wird im EPRI-Handbuch generell als Summe ihres elastischen und plastischen Anteils

$$
J=J_{e l}+J_{p l}
$$

in Abhängigkeit der äußeren Belastung $F$ und der Rissgröße $a$ beschrieben. Der elastische 
Anteil wird über die das elastische Verhalten beschreibende Größe $K_{I}$ mit

$$
J_{e l}=\frac{K_{I}^{2}\left(1-\nu^{2}\right)}{E}
$$

bestimmt. Der plastische Anteil wird mit folgender Gleichung beschrieben:

$$
J_{p l}=\alpha \cdot \varepsilon_{0} \cdot \sigma_{0} \cdot b \cdot h_{1}(a / W, N) \cdot\left(F / F_{\text {Grenz }}\right)^{N+1}
$$

Darin sind $\varepsilon_{0}, \sigma_{0}, \alpha$ und $N$ die Konstanten der Ramberg-Osgood-Beziehung

$$
\frac{\varepsilon}{\varepsilon_{0}}=\frac{\sigma}{\sigma_{0}}+\alpha\left(\frac{\sigma}{\sigma_{0}}\right)^{N}
$$

mit deren Hilfe die im Zugversuch ermittelte wahre Spannung-Dehnung-Beziehung beschrieben wird. Hierbei ist anzumerken, dass das Potenzgesetz nach Ramberg-Osgood problematisch ist, um das Werkstoffverhalten von Baustählen mit ausgeprägtem Lüdersplateau zu beschreiben.

Um die komplexe Beschreibung des J-Integrals von Kumar; German et al. (1981) bei der Untersuchung genieteter Bauteile in Stahlbrücken zu vermeiden, wurde von LANGENBERG (1996) und Stötzel (1998) der vereinfachte Potenzansatz nach Gleichung 2.48 basierend auf Berechnungen von Beltrami (1995) für die drei Modelle in Abbildung 2.16 verwendet.

$$
\frac{J}{J_{g y}}=m \cdot\left(\frac{F}{F_{g y}}\right)^{n}
$$

Die Rissspitzenbeanspruchung $J$ in Abhängigkeit der äußeren Zugkraft $F$ wird darin normiert mit der Beanspruchung $J_{g y}$ bei Erreichen der Nettoquerschnittsfließlast $F_{g y}$ (general yield). Die Größe $J_{g y}$ wurde ebenfalls für alle drei Modelle mittels FEM berechnet und in Abhängigkeit der Modellbreite $2 W$, der bezogenen Risslänge $a / W$ und der Streckgrenze $R_{e L}$ nach Gleichung 2.49 approximiert. Die Koeffizienten in den Gleichungen 2.48 und 2.49 sind in Tabelle 2.9 zusammengefasst.

$$
J_{g y}=\frac{2 W \cdot R_{e L}^{2}}{E} \cdot f \cdot \frac{a / W \cdot\left[1-(a / W)^{2}\right]}{a / W+k}
$$

Tab. 2.9.: Koeffizienten der Approximationsfunktionen der Rissspitzenbeanspruchung nach LANGENBERG (1996)

\begin{tabular}{c|c|c|c|c}
\hline Modell & $\mathrm{m}$ & $\mathrm{n}$ & $\mathrm{f}$ & $\mathrm{k}$ \\
\hline CCT & 0,95 & 2,8 & 0,625 & 0,135 \\
DECT & 1,05 & 2,0 & 0,693 & 0,165 \\
SECT & 0,95 & 2,9 & 2,062 & 0,198 \\
\hline
\end{tabular}

Für die bruchmechanische Bauteilauslegung sowie den Nachweis gegen sprödes Versagen anrissgefährdeter Bauteile existieren in den aktuell eingeführten Bewertungsvorschriften, wie z. B. der FKM-Richtlinie [Berger; Blauel et al. (2006)], BS 7910:2005 und SINTAP/FITNET [Bannister; Webster (1999)], sehr ähnliche Prozeduren. Alle Methoden 
haben den Vorteil, dass die in Abschnitt 2.6.2 beschriebenen analytischen, linear-elastischen K-Faktor-Lösungen auch für die elastisch-plastische Analyse von Baustahl verwendet werden können. Die Rissspitzenbeanspruchung wird in Abhängigkeit der Ausnutzung der Bauteilfließlast auf die für elastisch-plastisches Bauteilverhalten geltende Höhe korrigiert. Wie auch die Auftragung im CDF-Diagramm (siehe Abbildung 2.24) berücksichtigt die Bewertung mittels FAD (Failure Assessment Diagramm) die zwei möglichen Versagensmechanismen. Diese sind einerseits die Überschreitung der Bruchzähigkeit des Werkstoffs an der Rissspitze und Versagen durch instabiles Risswachstum. Andererseits begrenzt das FAD die ertragbare Beanspruchung des Bauteils gegen plastischen Kollaps, d.h. die Plastizierung des Ligaments.

Entsprechend der zur Verfügung stehenden Eingangsinformationen des zu bewertenden Werkstoffs bzw. der angestrebten Genauigkeit der Analyse bietet SINTAP verschiedene AnalyseEbenen (Optionen) für die bruchmechanische Bauteilbewertung. Unter der Voraussetzung, dass die Streckgrenze und die Zugfestigkeit des Grundmaterials wie für die hier zu untersuchenden Flussstähle bekannt ist, erfolgt die Bewertung auf der Analyse-Ebene 1A für einen Werkstoff mit Lüdersplateau. Die Grenzkurve des Failure Assessment Diagrams ist in Abhängigkeit der Ligamentplastifizierung $L_{r}=F / F_{Y}$ definiert zu:

$$
\begin{array}{ll}
f\left(L_{r}\right)=\left[1+0,5 \cdot L_{r}^{2}\right]^{-1 / 2} & \text { für } 0 \leq L_{r} \leq 1 \\
f\left(L_{r}\right)=\left[\lambda+\frac{1}{2 \lambda}\right]^{-1 / 2} & \text { für } L_{r}=1 \\
f\left(L_{r}\right)=f\left(L_{r}=1\right) \cdot L_{r}^{\frac{N-1}{2 N}} & \text { für } 1 \leq L_{r} \leq L_{r, \max }
\end{array}
$$

mit

$$
\lambda=1+\frac{E \cdot \Delta \varepsilon}{R_{e L}}
$$

Die Dehnung innerhalb des Lüdersplateaus kann entweder aus der experimentell ermittelten, wahren Spannungs-Dehnungs-Kurve entnommen oder nach SINTAP konservativ abgeschätzt werden mit:

$$
\Delta \varepsilon=0,0375 \cdot\left(1-\frac{R_{e L}}{1000 \mathrm{~N} / \mathrm{mm}^{2}}\right)
$$

Der Verfestigungsexponent $N$ ergibt sich ebenso aus der wahren Spannungs-Dehnungs-Kurve oder näherungsweise zu:

$$
N=0,3 \cdot\left(1-\frac{R_{e L}}{R_{m}}\right)
$$

Der obere Grenzwert der maximalen Ligamentplastizierung ist nach SINTAP definiert zu:

$$
L_{r, \max }=0,5 \cdot \frac{R_{e L}+R_{m}}{R_{e L}}
$$

Damit ergibt sich für die in dieser Arbeit untersuchten Flussstähle die in Abbildung 2.25 dargestellte FAD-Grenzkurve. 


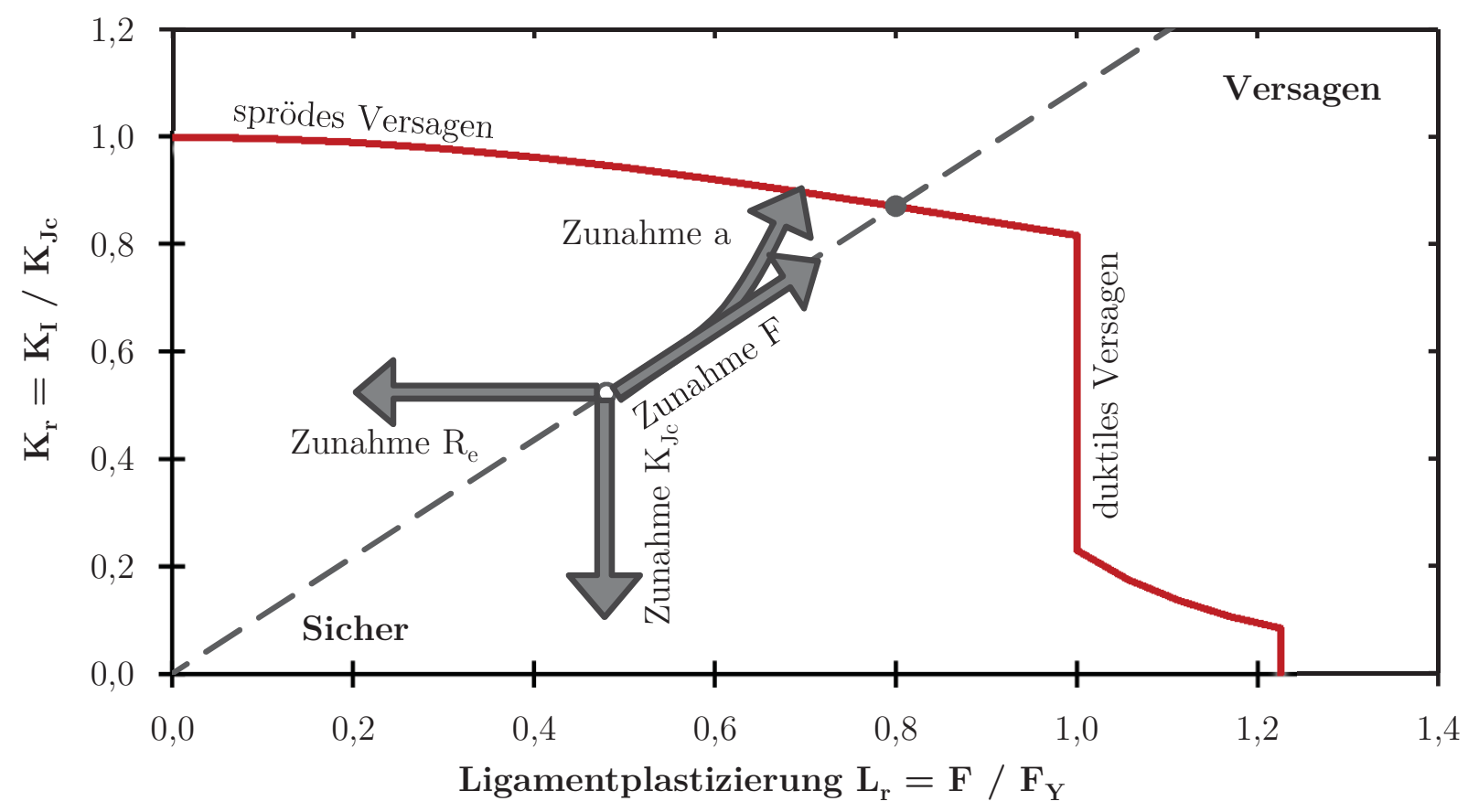

Abb. 2.25.: Bruchsicherheitsbewertung auf Basis des FAD Option 1A nach SINTAP [BAnNister; WEBSTER (1999)]

\subsubsection{Plastische Grenzlasten für Lochstäbe}

Eine möglichst genaue Beschreibung der plastischen Grenzlast ist wichtig für eine zuverlässige Versagensanalyse mit dem FAD. Für Standardgeometrien wurden entsprechende Lösungen hergeleitet und in Kompendien zur Verfügung gestellt, z. B. FKM-Richtlinie [BERGER; Blauel et al. (2006)], BS 7910:2005 und FITNET Al Laham; Ainsworth et al. (1998). Wie bei LANGenberg (1996) und Stötzel (1998) werden im Rahmen dieser Arbeit für die Untersuchung gelochter Bauteile die folgenden Ansätze zur Bestimmung der plastischen Grenzlasten verwendet. Sie basieren auf FEM-Berechnungen der Modelle nach Abbildung 2.16 für den ebenen Spannungszustand. Die Annahme des ESZ ist zum einen angesichts der geringen Materialdicken in alten Stahlkonstruktionen gerechtfertigt, wird darüber hinaus aber auch im Sinne eines zuverlässigen bruchmechanischen Nachweises z. B. von ZERBST; SchÖDEL et al. (2007) und VERSCH (2014) empfohlen.

Für den Lochstab mit symmetrischem Mittenriss (CCT) geben EHrHardT (1988) und KuMAR; GERMAn et al. (1981) an:

$$
\sigma_{Y, C C T}=R_{e} \cdot\left(1-\frac{2 a}{W}\right)
$$

Die plastische Grenzlast des Lochstabes mit beidseitig symmetrischem Randriss (DECT) ergibt sich nach STöTzEL (1998) zu:

$$
\sigma_{Y, D E C T}=R_{e} \cdot\left(1-\frac{2 a}{W}\right) \cdot\left(1+0,25 \cdot \frac{2 a}{W}\right)
$$


Für die endlich breite Scheibe mit einseitigem Durchriss (SECT) bestimmt Silcher (1991) die Grenzlast mit:

$$
\sigma_{Y, S E C T}=R_{e} \cdot\left(1-\frac{a}{W}\right)
$$

Die Ermittlung von Grenzlast-Lösungen erfolgte über die letzten Jahrzehnte mittels FEM und verschiedenen anderen Methoden. Entsprechend unterschiedlich ist ihre Genauigkeit. Die Folge ist eine mehr oder weniger konservative Lösung der bruchmechanischen Analyse. Für spezielle, besonders kritische Anwendungen gehen z. B. ZERBst; Ainsworth et al. (2010) und Madia; Arafah et al. (2014) dazu über, die plastische Grenzlast im Sinne der Referenzlast nach Ainsworth (1984) zu bestimmen. Diese wird für die betrachtete Bauteilgeometrie und Risskonfiguration, unter Ansatz realen Werkstoffverhaltens mittels FEM, aus der Bedingung abgeleitet, dass der Ligamentplastizierungsparameter $L_{r}$ der $f\left(L_{r}\right)$ Funktion genau 1 beträgt. Angesichts der erheblichen Streuungen des Spannungs-DehnungsVerhaltens alter Flussstähle (siehe Abbildung 3.3) ist die Ermittlung einer allgemeingültigen Referenzlastlösung nach diesem Ansatz nicht sinnvoll möglich. Die Auswertungen in Kapitel 5 zeigen außerdem, dass für die in dieser Arbeit untersuchten Lochstäbe die o. g. Ansätze zur Bestimmung der plastischen Grenzlast eine ausreichende Genauigkeit aufweisen. 


\section{Kapitel 3.}

\section{Experimentelle Untersuchungen an alten Baustählen}

\subsection{Vorbemerkungen}

In diesem Kapitel werden die im Zusammenhang mit dieser Arbeit durchgeführten experimentellen Untersuchungen sowie die Auswertung der Ergebnisse der Werkstoffuntersuchungen beschrieben. $\mathrm{Zu}$ den Voruntersuchungen für die Finite-Element-Berechnungen gehören die Bestimmung der Werkstoffkenndaten alter Baustähle (siehe Abschnitt 3.3) und detaillierte Analysen der Werkstoffeigenschaften an den Rändern gestanzter Löcher, um die verfestigenden Einflüsse aus dem Stanzprozess zu erfassen (siehe Abschnitt 3.5).

Der Schwerpunkt dieses Kapitels liegt auf der Bestimmung der Werkstoffzähigkeit alter Baustähle und der Überprüfung möglicher Zusammenhänge zu deren Herstellung. Die Untersuchungen sind in Abschnitt 3.4 dargestellt. Zur Verifizierung des in Kapitel 5 erläuterten Sicherheitsnachweises werden abschließend in Abschnitt 3.6 Bauteilzugversuche bei tiefen Temperaturen erläutert, mit denen das Sprödbruchverhalten von Bauteilen aus alten Baustählen analysiert wird.

\subsection{Probenmaterial und Werkstoffeigenschaften}

Wie aus den Untersuchungen von MAng; BuCAK (1996), LANGEnBERG (1996), Reiche (2000), Käpplein; Wielgosch-Frey (2001) und Helmerich (2005) bereits bekannt ist, sind die metallurgischen und mechanischen Eigenschaften alter Baustähle erheblichen Streuungen unterlegen. Um die verschiedenen Werkstoffeigenschaften der Werkstoffgruppe „Flussstahl“ abzubilden, wurden Werkstoffproben verschiedener Baujahre aus unterschiedlichen Bauwerken und mehreren Querschnittstypen für die Analysen ausgewählt. Da sich erfahrungsgemäß heute wie früher die in Konstruktionen des Hochbaus verwendeten Stähle qualitativ (Reinheitsgrad, Festigkeit, Zähigkeit) von den im Brückenbau eingesetzten Stählen unterscheiden, wurden ausschließlich Materialproben aus bestehenden Stahlhochbaukonstruktionen untersucht. Wesentliche Informationen zu den gewählten Werkstoffproben, wie deren Herkunft, Bauteil, Alter und Profilquerschnitt enthält Tabelle 3.1. 
Tab. 3.1.: Probenmaterial für die Untersuchungen der Werkstoffzähigkeit

\begin{tabular}{l|c|c|c}
\hline Bauwerk / Bauteil & Baujahr & Bezeichnung & Profil \\
\hline Heizkraftwerk DD Nord / Deckenträger & $1913 / 18$ & DT200 & I200 \\
Heizkraftwerk DD Nord / Deckenträger & $1913 / 18$ & DT260 & I260 \\
Stahlgittermast M31 / Eckstiel & 1930 & M31 & L120x13 \\
Stahlgittermast M56 / Eckstiel & 1930 & M56 & L110x12 \\
Stahlgittermast / Eckstiel & 1921 & SGM21 & L80x8 \\
Personenaufzug Bad Schandau / Diagonale & 1904 & PA2 & L60x8 \\
Bf Bad Kreuznach / Stützengurt & 1907 & DB_G1 & L100x65x11 \\
Bf Bad Kreuznach / Stützengurt & 1907 & DB_G3 & L100x65x11 \\
\hline
\end{tabular}

\subsubsection{Chemische und metallografische Analyse}

Die chemischen Analysen erfolgten mit einem Funkenspektrometer jeweils an den Querschnittsflächen der Proben, um die inhomogene Verteilung einzelner Begleitelemente aufgrund von Seigerungen mit zu erfassen. Zur Bestimmung der gasförmigen Bestandteile Stickstoff und Sauerstoff wurden ergänzend Analysen mittels Trägergas-Heißextraktion durchgeführt. Die Analyseergebnisse des Probenmaterials sind in Tabelle 3.2 zusammengefasst und den mittleren Konzentrationen typischer Flussstähle gegenübergestellt. Das Versuchsmaterial enthält stickstoffreiche Konverterstähle (z. B. PA2) ebenso wie Siemens-Martin-Stähle (z. B. M31).

Die Gehalte an Eisenbegleitern im untersuchten Probenmaterial entsprechen den üblichen Konzentrationen in Massenbaustählen aus den Anfängen des letzten Jahrhunderts. Wie am Silizium-Gehalt zu erkennen ist, handelt es sich bei allen Werkstoffproben um unberuhigt vergossene Stähle. Der Gehalt an Aluminium wurde im Rahmen der durchgeführten Vollanalysen ebenfalls bestimmt, aber hier nicht angegeben, da eine Legierung mit Aluminium erst Mitte der 1930er Jahre durchgeführt wurde (siehe Abschnitt 2.1).

Tab. 3.2.: Ergebnisse der chemischen Analysen, Referenzwerte nach ReIche (2000)

\begin{tabular}{l|c|c|c|c|c|c|c}
\hline \multirow{2}{*}{ Probe } & \multicolumn{7}{|c}{ Chemische Analyse [\%] } \\
& $\mathrm{C}$ & $\mathrm{Mn}$ & $\mathrm{Si}$ & $\mathrm{P}$ & $\mathrm{S}$ & $\mathrm{N}$ & $\mathrm{O}$ \\
\hline DT200 & 0,031 & 0,27 & 0,001 & 0,049 & 0,029 & 0,0135 & 0,0110 \\
DT260 & 0,097 & 0,72 & 0,001 & 0,095 & 0,102 & 0,0250 & 0,0155 \\
M31 & 0,072 & 0,48 & 0,001 & 0,024 & 0,043 & 0,0115 & 0,0385 \\
M56 & 0,150 & 0,36 & 0,001 & 0,018 & 0,091 & 0,0080 & 0,0100 \\
SGM21 & 0,088 & 0,23 & 0,001 & 0,087 & 0,089 & 0,0210 & 0,0160 \\
PA2 & 0,067 & 0,67 & 0,001 & 0,103 & 0,079 & 0,0225 & 0,0150 \\
DB_G1 & 0,029 & 0,32 & 0,002 & 0,053 & 0,085 & 0,0250 & 0,0710 \\
DB_G3 & 0,038 & 0,41 & 0,001 & 0,060 & 0,084 & 0,0190 & 0,0570 \\
\hline Thomas-Stahl & 0,048 & 0,46 & 0,009 & 0,051 & 0,044 & 0,0140 & - \\
Siemens-Martin-Stahl & 0,090 & 0,48 & 0,008 & 0,035 & 0,038 & 0,0050 & - \\
\hline
\end{tabular}


Als ergänzender Nachweis der Seigerungen sowie als Grundlage für die anschließende Probenfertigung, wurden an allen Materialproben Baumannabdrücke am Profilquerschnitt hergestellt. Mit der auch als Schwefelabdruck bezeichneten Makroätzung wird die Verteilung von Fe- und Mn-Sulfideinschlüssen im Stahl sichtbar gemacht [PETzOw (1976)].

\subsubsection{Festlegung der Probengeometrie und Probenherstellung}

Ausgehend von den zu ermittelnden Werkstoffkennwerten und den dafür anzuwendenden Prüfverfahren wurden für jede zu analysierende Werkstoffprobe folgende Probengeometrien und -anzahlen festgelegt:

- Zugversuch: 6 Stück Rundzugprobe B5 (Messlänge 25 mm) nach DIN 50125:2009,

- Kerbschlagbiegetest: 12 Stück 0,4T-ISO-V-Probe nach DIN EN ISO 148-1:2011,

- Bruchmechaniktest: 10 Stück 0,5T- oder 0,25T-C(T)-Probe nach ASTM E 1820:2013.

Grundlage für die Probenentnahme aus dem zur Verfügung stehenden Profilmaterial bildeten die vorab angefertigten Baumannabdrücke, mit deren Hilfe die aus dem Herstellungsprozess herrührenden Schwefelseigerungen sichtbar gemacht wurden. Die Entnahme der Proben erfolgte ausschließlich im Bereich dieser Seigerungen, da in diesen Teilen des Querschnitts, aufgrund der erhöhten Menge an Verunreinigungen, die repräsentative Werkstoffzähigkeit zu erwarten war. Alle Proben eines Typs wurden, wenn möglich, so entnommen, dass sie in Profillängsrichtung hintereinander lagen und dadurch Material mit annähernd gleichen Eigenschaften untersucht werden konnte. Stand von einer Werkstoffprobe nicht ausreichend Material zur Verfügung, um alle Kerbschlagproben in einem Strang zu entnehmen, wurde direkt daneben eine zweite Reihe so angeordnet, dass die Kerben der Proben unmittelbar gegenüber lagen. Wies eine Werkstoffprobe eine Materialdicke von weniger als $10 \mathrm{~mm}$ auf, so wurden die Kerbschlagproben nicht mit dem von DIN EN ISO 148-1:2011 vorgeschlagenen Untermaß von 7,5 mm oder 5,0 mm gefertigt, sondern stets die maximal mögliche Probenbreite ausgeführt.
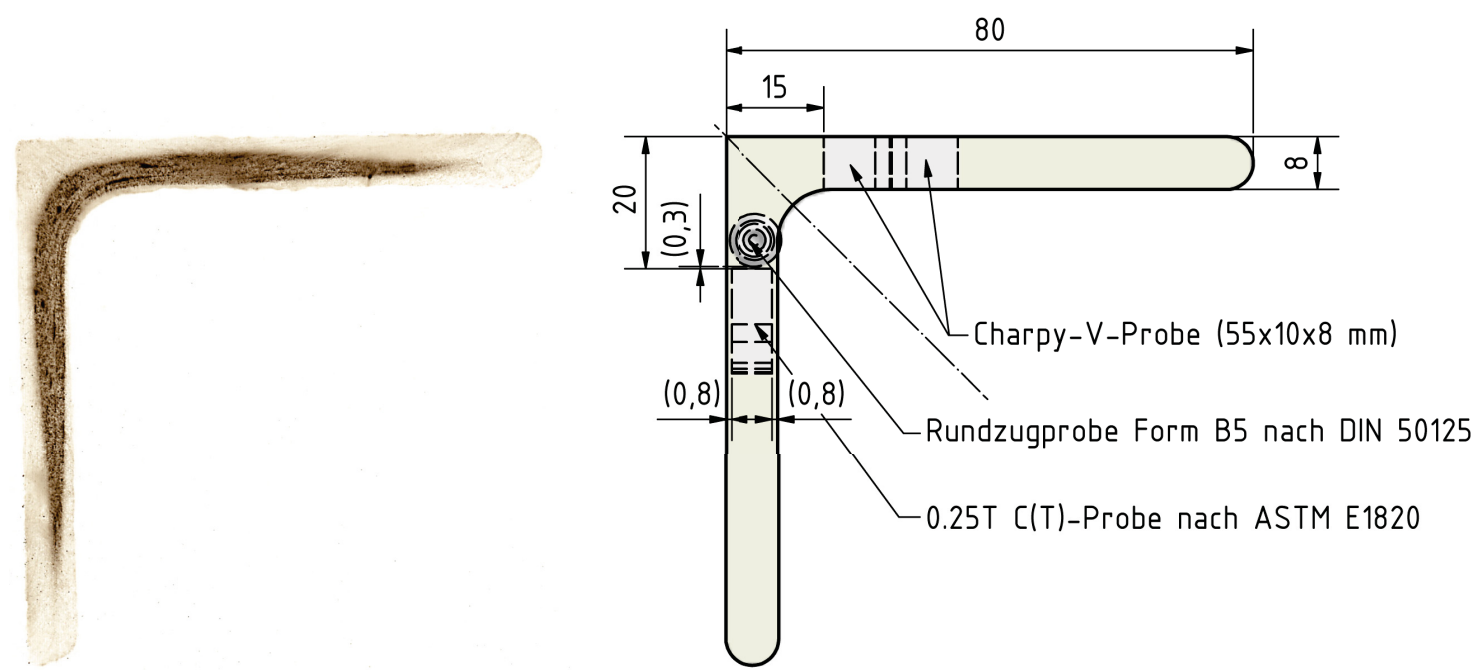

Abb. 3.1.: Baumannabdruck und Schnittplan der Probe SGM21 [Stroetmann; Sieber et al. (2015)] 
Es wurde darüber hinaus darauf geachtet, die Proben so aus dem jeweiligen Querschnitt zu entnehmen, dass sich der Kerb bzw. der Ermüdungsriss in einer Zone des Querschnitts befand, wo üblicherweise Nietlöcher vorhanden und damit auch Anrisse in den Bauteilen zu erwarten sind (siehe Abbildung 1.1). Abbildung 3.1 zeigt den Baumannabdruck einer Werkstoffprobe zusammen mit dem daraus abgeleiteten Schnittplan der Probekörper.

\subsection{Festigkeitskennwerte}

Für die Auswertung der Bruchmechaniktests nach dem Master-Curve-Konzept (siehe Abschnitt 3.4) wie auch für eine Klassifizierung der untersuchten alten Baustähle im Allgemeinen wurden die mechanischen Werkstoffkennwerte in mindestens 6 Zugversuchen ermittelt. Im Hinblick auf den Einsatztemperaturbereich der Stähle wurde festgelegt, je zwei Tests bei Raumtemperatur, bei $-10^{\circ} \mathrm{C}$ und bei $-30{ }^{\circ} \mathrm{C}$ durchzuführen. Die Durchführung der Zugversuche bei Raumtemperatur erfolgte nach DIN EN ISO 6892-1:2009, bei Temperaturen unterhalb RT nach DIN ISO 15579:2002. Die Ergebnisse der Versuche bei Raumtemperatur sind in Tabelle 3.3 den Mittelwerten typischer Flussstähle aus der Literatur gegenübergestellt.

Um die bestimmten Festigkeitskennwerte im Rahmen der Master-Curve-Auswertung auch auf Bruchzähigkeitswerte anwenden zu können, die bei abweichenden Prüftemperaturen bestimmt worden sind, wurden die Einzelwerte der verschiedenen Temperaturen zunächst nach dem bei MARGolin; Gulenko et al. (2005) verwendeten Exponentialansatz (Gleichung 3.1) approximiert:

$$
R_{e L, m}=A+B \cdot e^{(-h \cdot(T+273))}
$$

Die Versuchsergebnisse der einzelnen Flussstahlproben sowie die zugehörigen Kurvenverläufe sind in den Abbildungen A.1 und A.2 dargestellt. Es wird deutlich, dass die Abhängigkeit der Streckgrenze und Zugfestigkeit von der Temperatur im untersuchten Temperaturbereich annähernd linear ist. Diesen Zusammenhang bestätigen auch die Ergebnisse von LANGENBERG (1996), die den hier gewonnenen Daten in Abbildung 3.2 gegenübergestellt sind. Da es

Tab. 3.3.: Mittlere Festigkeitskennwerte bei RT im Vergleich zu Angaben aus der Literatur

\begin{tabular}{l|c|c|c}
\hline Probe & $R_{e l}\left[\mathrm{~N} / \mathrm{mm}^{2}\right]$ & $R_{m}\left[\mathrm{~N} / \mathrm{mm}^{2}\right]$ & $A[\%]$ \\
\hline DT200 & 260,0 & 385,8 & 28,8 \\
DT260 & 303,3 & 433,0 & 28,1 \\
M31 & 289,2 & 397,9 & 28,4 \\
M56 & 301,3 & 437,6 & 26,4 \\
SGM21 & 327,8 & 430,3 & 27,0 \\
PA2 & 344,9 & 477,6 & 27,1 \\
DB_G1 & 242,4 & 346,0 & 38,8 \\
DB_G3 & 249,2 & 354,6 & 39,8 \\
\hline Mittelwert aller Proben & 289,8 & 407,9 & 30,6 \\
\hline Mittelwert nach ReICHE (2000) & 268 & 393 & 34,8 \\
Mittelwert nach LANGEnBERG (1996) & 282 & 417 & 27,0 \\
\hline
\end{tabular}


sich bei den dort untersuchten Flussstählen nahezu ausschließlich um Material aus alten Eisenbahnbrücken mit üblicherweise besserer Werkstoffgüte handelt, liegen die Festigkeiten der hier untersuchten Altstähle geringfügig höher. Da die von LANGENBERG (1996) aufgestellten Approximationen

$$
\begin{aligned}
& R_{e L}=271,4-0,68 \cdot T \\
& R_{m}=393,7-0,68 \cdot T
\end{aligned}
$$

auf einer wesentlich größeren Menge an Versuchsergebnissen basieren und sie das Werkstoffverhalten im interessierenden Temperaturbereich ausreichend genau beschreiben, werden diese bei der Erarbeitung des Bruchmechanischen Nachweiskonzepts in Kapitel 5 verwendet.

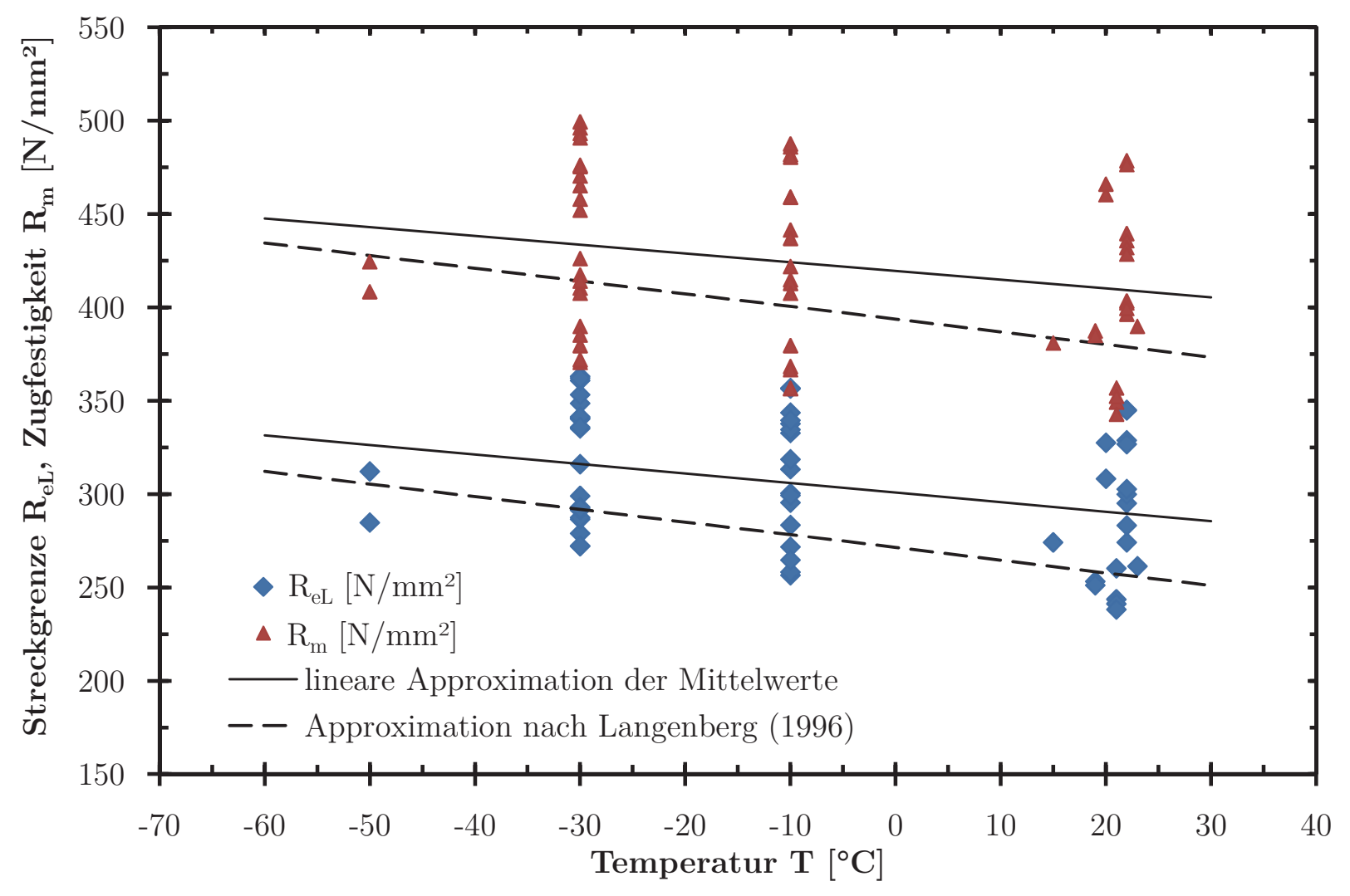

Abb. 3.2.: Untere Streckgrenze $R_{e L}$ und Zugfestigkeit $R_{m}$ in Abhängigkeit der Temperatur $T$

Um darüber hinaus das elastisch-plastische Werkstoffverhalten der untersuchten Flussstähle mit den Ergebnissen früherer Untersuchungen vergleichen zu können, wurden während der Zugversuche die Längs- und Querdehnung der Zugproben mit einem Videoextensometer gemessen. Mit Hilfe dieser Messdaten konnten anschließend wahre Spannungs-DehnungsKurven (siehe [Blumenauer (1994)]) der einzelnen Werkstoffproben ermittelt werden. Abbildung 3.3 enthält die wahren Spannungs-Dehnungs-Kurven der Zugversuche aller untersuchter Flussstahlproben.

Den Messergebnissen ist die von Langenberg (1996) gewählte Approximation

$$
\sigma=\sigma_{0}+C \cdot \varepsilon^{n}
$$

gegenübergestellt. Sie basiert auf einem modifizierten Potenzansatz nach Ludwik, der neben der Verfestigung auch die Streckgrenze $\sigma_{0}$ berücksichtigt. Alternativ wurde mit Gleichung 


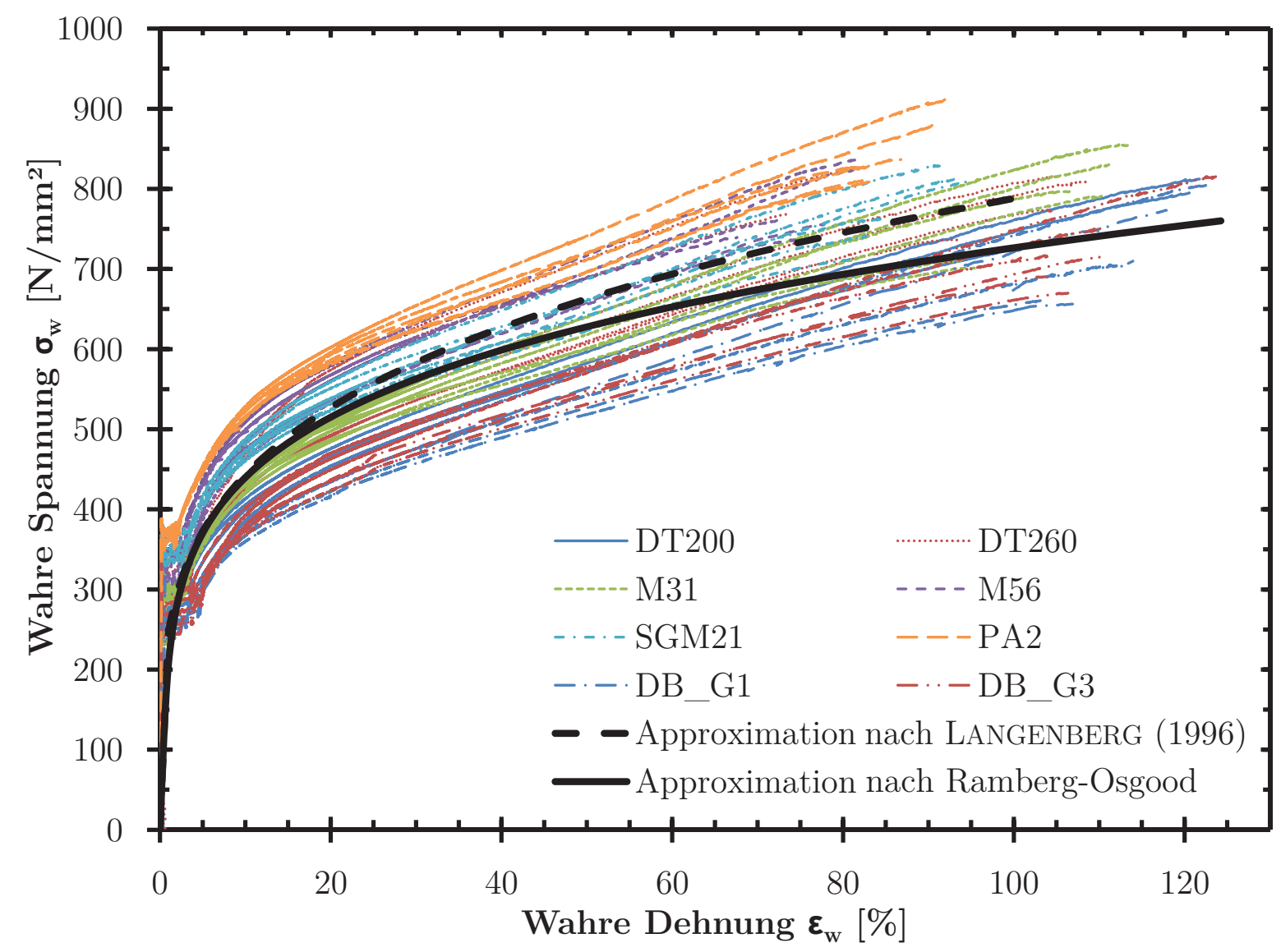

Abb. 3.3.: Wahre Spannungs-Dehnungskurven der untersuchten Flussstähle

3.5 ein Potenzansatz nach Ramberg-Osgood in einer nach BLumenauer (1994) modifizierten Form gewählt. Diese Approximation folgt im Mittel den wahren Spannungs-DehnungsKurven der untersuchten Flussstähle bis zu einer Dehnung von etwa $40 \%$ besser als die Ludwik-Gleichung. Das Verfestigungsverhalten wird hingegen konservativ unterschätzt.

$$
\frac{\varepsilon}{\varepsilon_{0}}=\frac{\sigma}{\sigma_{0}}+\frac{3}{7}\left(\frac{\sigma}{\sigma_{0}}\right)^{m}
$$

\subsection{Werkstoffzähigkeiten}

\subsubsection{Kerbschlagzähigkeit}

Wie bereits in Abschnitt 2.5 erläutert, eignet sich der Kerbschlagbiegeversuch nicht zur Beurteilung der Sprödbruchneigung alter Baustähle, da entsprechende Erfahrungen bzw. experimentell und analytisch abgeleitete Mindestwerte der Übergangstemperatur, wie sie für heutige Baustähle in DIN EN 1993-1-10:2010 angegeben sind, noch nicht oder nur unzureichend existieren. Um zukünftig eine Prüfung ausreichender Werkstoffzähigkeit mit diesem technologisch sehr einfachen Versuch zu ermöglichen, erfolgten umfangreiche Untersuchungen der Kerbschlagzähigkeit der zu Beginn des Kapitels beschriebenen alten Baustähle. Um 
die Aussagefähigkeit des Kerbschlagbiegeversuchs gegenüber der gewöhnlichen Versuchsanordnung wesentlich zu erweitern, wurden die Versuche ausschließlich nach DIN EN ISO 14556:2006 instrumentiert durchgeführt. Dadurch lassen sich neben der Schlagarbeit als zusätzliche Versuchsgrößen Kraft, Durchbiegung und Zeit aufnehmen. Weiterhin ermöglicht eine Instrumentierung die Ermittlung des momentanen Wertes genannter Größen während des Versuchs (siehe Abbildung 3.4). Dies ist gerade bei markanten Änderungen des Materialverhaltens wichtig, wie z. B. beim Wechsel des Bruchmechanismus beim Erreichen der Rissauffangkraft $F_{a}$.

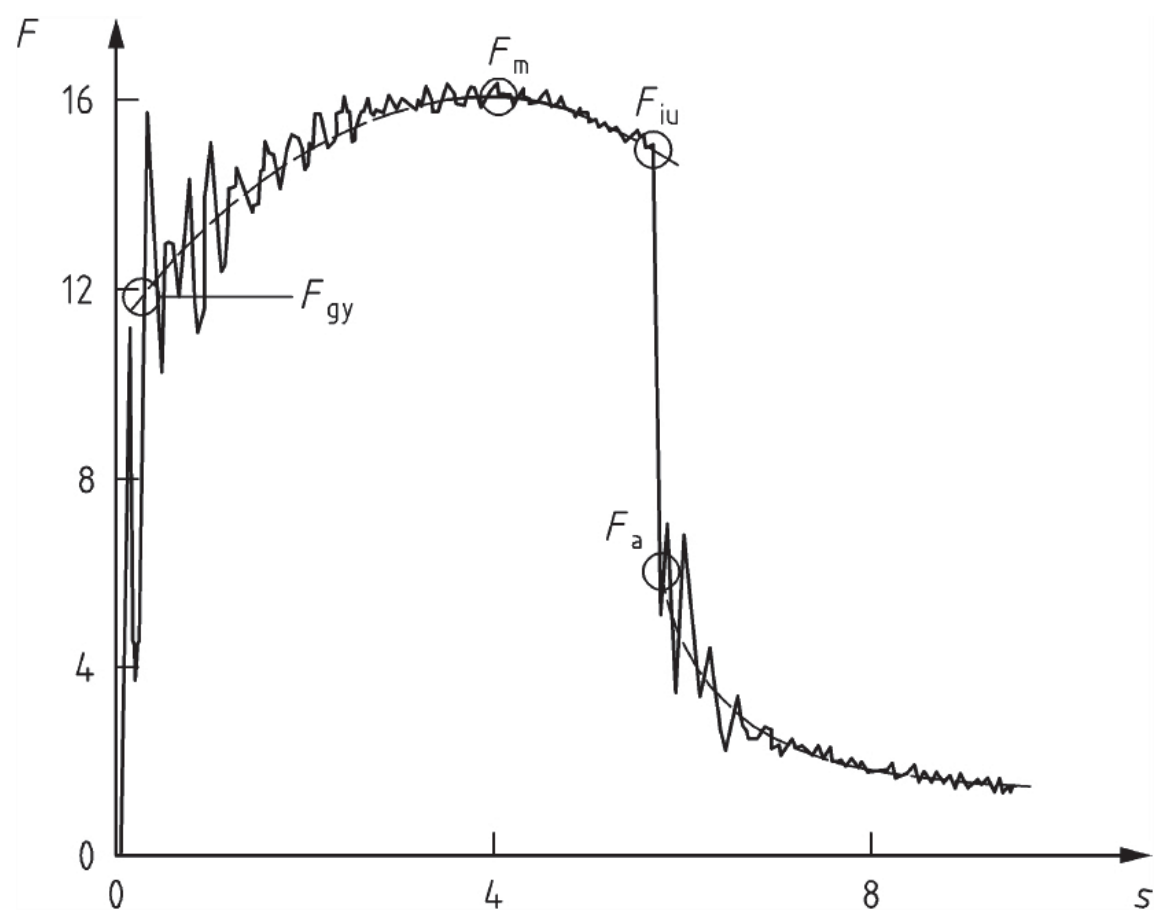

Abb. 3.4.: Kenngrößen im Schlagkraft-Durchbiegungs-Diagramm des instrumentierten Kerbschlagbiegeversuchs nach DIN EN ISO 14556:2006

Von jeder Werkstoffprobe wurden mindestens 12 Kerbschlagproben bei verschiedenen Temperaturen untersucht. Die Festlegung des Prüftemperaturbereichs erfolgte operativ für jedes Versuchsmaterial anhand der ersten Testergebnisse. Die in Abhängigkeit der Temperatur gemessenen Kerbschlagarbeiten $K V_{2}$ der untersuchten Flussstahlproben zeigen die Abbildungen A.3 bis A.10 in Anhang A. Die Datenpunkte wurden mit einer Tangens-HyperbolicusFunktion approximiert:

$$
K V_{2}=A+B \cdot \tanh \left(\frac{T-T_{0}}{C}\right)
$$

und daraus die spröd-duktilen Übergangstemperaturen sowie die Kerbschlagarbeit in der Zähigkeitshochlage für die einzelnen Materialproben ermittelt. In Tabelle 3.4 sind die Ergebnisse dieser Auswertungen zusammengefasst.

Die Kerbschlag-Temperaturkurven aller 8 untersuchten Flussstahlproben sind in Abbildung 3.5 zusammenfassend gegenübergestellt. Diese Abbildung verdeutlicht noch einmal die Streuungen der Kerbschlagzähigkeit innerhalb der Werkstoffgruppe Flussstahl. Außerdem bestätigt sie die Erkenntnis, dass sich die Übergangstemperaturkurve dieser Stähle bei Raumtemperatur häufig schon in der Tieflage oder dem unteren Übergangsbereich befindet. Die überdurchschnittlich hohen Kerbschlagarbeiten der Werkstoffprobe DT200 in der Hochlage sind 
Tab. 3.4.: Ergebnisse der Kerbschlagbiegeversuche

\begin{tabular}{l|c|c|c|c|c|c}
\hline \multirow{2}{*}{ Probe } & \multirow{2}{*}{$T_{27 J}\left[{ }^{\circ} \mathrm{C}\right]$} & $K V_{2, \text { Hochlage }}[\mathrm{J}]$ & \multicolumn{4}{|c}{ Parameter der Approximation } \\
& & $A[\mathrm{~J}]$ & $B[\mathrm{~J}]$ & $C\left[{ }^{\circ} \mathrm{C}\right]$ & $T_{0}\left[{ }^{\circ} \mathrm{C}\right]$ \\
\hline DT200 & 38,3 & 272,0 & 142,0 & 130,0 & 4,1 & 44,0 \\
DT260 & 26,4 & 114,3 & 56,7 & 57,6 & 28,5 & 42,6 \\
M31 & 40,5 & 139,8 & 71,9 & 67,8 & 21,7 & 57,8 \\
M56 & 37,1 & 77,3 & 40,3 & 37,0 & 27,8 & 47,6 \\
SGM21 & 17,1 & 101,7 & 51,7 & 50,0 & 15,3 & 25,3 \\
PA2 & 14,4 & 114,1 & 58,6 & 55,5 & 19,3 & 26,8 \\
DB_G1 & 25,1 & 145,0 & 75,8 & 69,3 & 22,5 & 44,7 \\
DB_G3 & $-0,5$ & 132,7 & 67,3 & 65,4 & 38,3 & 27,0 \\
\hline
\end{tabular}

auf die niedrigen Gehalte von Eisenbegleitern (siehe Tabelle 3.2) und die dementsprechend nur schwach ausgeprägten Seigerungen im Bereich der Probenentnahme zurückzuführen.

Bei den Werkstoffproben SGM21, PA2 und DB_G3 (Abbildungen A.7, A.8 und A.10) weist die gemessene Kerbschlagarbeit in der Hochlage einen unerwarteten Anstieg mit wachsender Temperatur auf. Dies äußert sich auch in deutlichen Unterschieden der Kraft-Durchbiegungs-

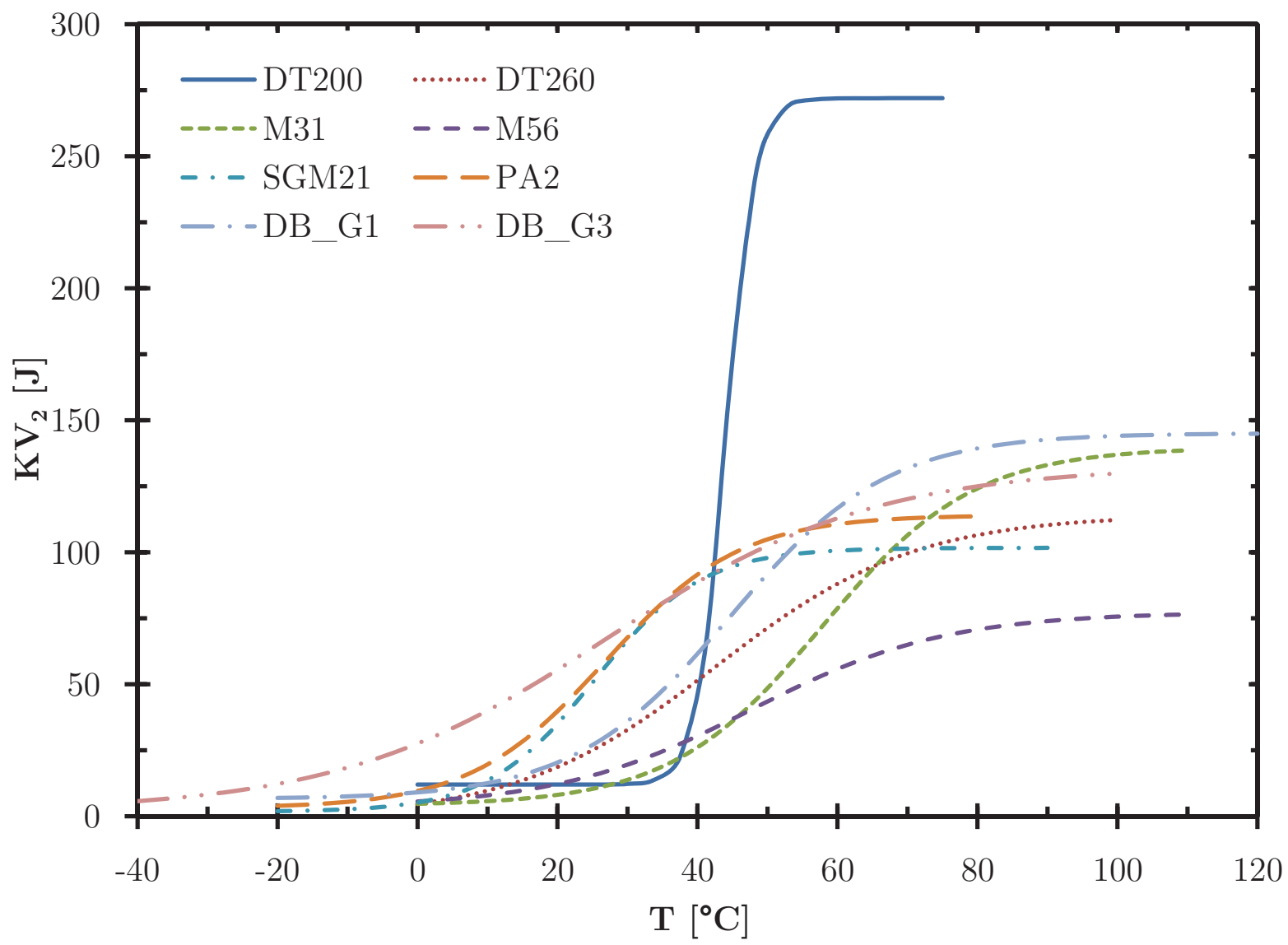

Abb. 3.5.: Kerbschlag-Temperaturkurven aller untersuchten Flussstahlproben 
Kurven von vollständig duktil versagten Proben (entspricht Typ F nach Bild 2 in DIN EN ISO 14556:2006). Abbildung 3.6 zeigt exemplarisch zwei Kurven des Bruchtyps F der Werkstoffprobe PA2. Dieses Ergebnis weist auf einen inhomogenen Werkstoffzustand hin. Die Unterschiede im plastischen Verformungsvermögen sind nicht allein mit der Prüftemperatur begründbar. Die Vermutung einer Verfestigung des Probenmaterials, durch künstliche Alterung bei höheren Temperaturen, konnte durch Zugversuche an gealterten Zugproben nicht bestätigt werden.

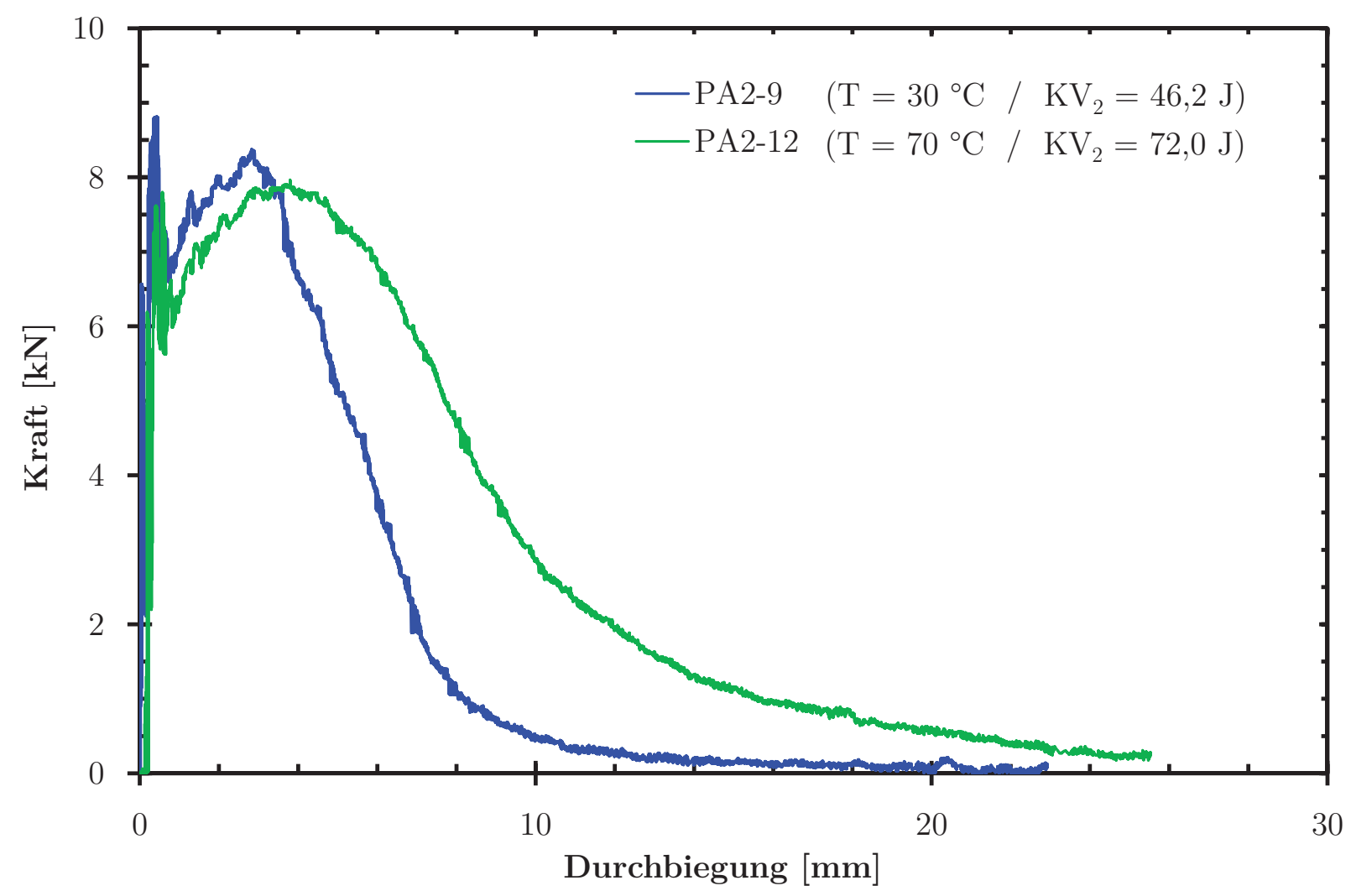

Abb. 3.6.: Kraft-Durchbiegungskurven vollständig duktil versagter Kerbschlagbiegeproben (Typ F) aus Werkstoffprobe PA2

\subsubsection{Vergleich mit Kerbschlagzähigkeiten alter Brückenbaustähle}

Zur Validierung der zuvor dargestellten Versuchsergebnisse erfolgte eine Gegenüberstellung mit statistischen Auswertungen von LAngenBerg (1996), auf Basis von Untersuchungsergebnissen des IEHK Aachen für alte Flussstähle des Brückenbaus (siehe Abbildung 3.7).

Die hier untersuchten Flussstähle des Hochbaus liegen nahezu vollständig innerhalb der einfachen Standardabweichung, allerdings im Übergangs- und Hochlagenbereich überwiegend unterhalb des angegebenen Mittelwerts. Da es sich bei den Ergebnissen von LANGenBerG (1996) um Stähle aus einem alten Brückenbauwerk handelt, ist die niedrige Zähigkeit der hier untersuchten Stähle sehr wahrscheinlich auf die geringeren Werkstoffgüten im Stahlhochbau 


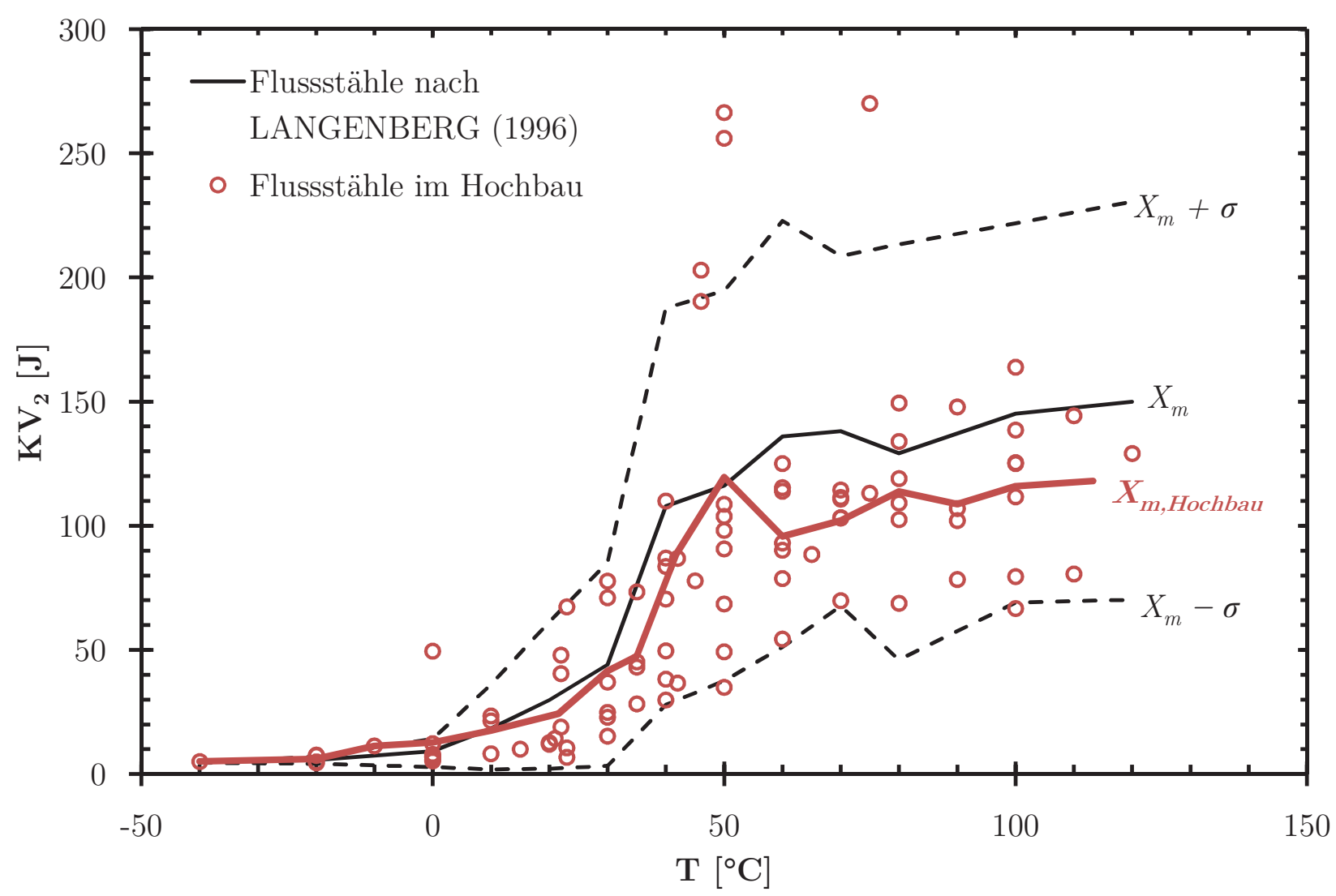

Abb. 3.7.: Kerbschlagarbeit der untersuchten Flussstahlproben im Vergleich zu Auswertungen an Flussstählen im Brückenbau durch LANGENBERG (1996)

zurückzuführen, auf die bereits in Kapitel 1 hingewiesen wurde. Da der Ort der Probenentnahme im Bauteil und die Orientierung der Proben bei den von Langenberg ausgewerteten Kerbschlagbiegeversuchen unzureichend dokumentiert ist, sind die geringen Kerbschlagzähigkeiten der Hochbaustähle u. U. auch darauf zurückzuführen, dass die im Rahmen dieser Arbeit untersuchten Proben generell aus den stark verunreinigten Seigerungszonen der Bauteile entnommen wurden.

\subsubsection{Bruchmechanische Zähigkeit - Bestimmung der Referenztemperatur nach dem Master-Curve-Konzept}

Da sich die zu Beginn des Abschnitts 3.4 beschriebene Kerbschlagzähigkeit nicht für eine quantitative Beurteilung der Sprödbruchneigung alter Baustähle im Sinne einer Nachweisgleichung eignet, ist eine bruchmechanische Betrachtung des Sprödbruchproblems und damit eine Bestimmung der Bruchzähigkeit der alten Baustähle erforderlich.

Das bereits in Kapitel 2.4 erläuterte Bruchverhalten der Baustähle führt zu einem temperaturabhängigen Übergang der Werkstoffzähigkeit vom duktilen Zustand („Hochlage“) zum spröden Zustand („Tieflage“). Dieser Übergangsbereich ist durch deutlich größere Streuungen der Zähigkeit als das Hoch- und Tieflagengebiet gekennzeichnet. Dieses breite Streuband wird für die Bruchzähigkeit mit dem Weakest-Link-Modell nach LANDES; SHAfFer (1980) 
erklärt. Danach entscheidet eine Gefügeschwachstelle an der Rissfront als das „schwächste Glied in der Kette" über die Zuverlässigkeit des gesamten Bauteils. An diesen Gefügeschwachstellen bilden sich in der Regel Mikrorisse, die sich instabil ausbreiten und damit zum Strukturversagen führen. Ursache des breiten Streubands ist die stochastische Verteilung der Gefügeschwachstellen im Ligament. Je näher sich die Schwachstelle an der Rissfront befindet, desto geringer ist die Bruchzähigkeit der Probe. Ebenso gilt, je länger die Rissfront, umso höher ist die Wahrscheinlichkeit, dass eine den Spaltbruch auslösende Schwachstelle in der Nähe der Rissfront liegt. Aus diesem Grund ist bei dickeren Proben der Risswiderstand niedriger als bei dünneren Proben. Die Streuungen der zugehörigen Risszähigkeit sind dafür aber geringer. Im Tieflagenbereich tritt dieser Geometrieeffekt allerdings nicht auf [LANDEs; HeErens et al. (1993)].

Darauf aufbauend wird im Master Curve-Konzept nach WALlin (1984), WaLlin (1985), WALLIN (1998) der Risswiderstand im spröd-duktilen Übergangsbereich in probabilistischer Form mit Hilfe der Weibullstatistik formuliert. Die Versagenswahrscheinlichkeit $P_{f}$ folgt demnach einer dreiparametrigen Weibullverteilung, wobei zwei der drei Parameter fest definiert sind. Der Formparameter (shape parameter) beträgt $m=4$, und der Schwellenwertparameter (threshhold parameter) begrenzt die Tieflage der Bruchzähigkeit ferritischer Stähle auf $K_{\text {min }}=20 \mathrm{MPa} \sqrt{\mathrm{m}}$ :

$$
P_{f}=1-\exp \left(-\left(\frac{K_{J c}-K_{\min }}{K_{0}-K_{\min }}\right)^{m}\right)
$$

Einzig der Skalenparameter (scale parameter) $K_{0}$ wird anhand der bei instabilem Versagen der Proben ermittelten Bruchzähigkeitswerten $K_{J c}$ mit:

$$
K_{0}=\left[\sum_{i=1}^{N} \frac{\left(K_{J c(i)}-K_{\min }\right)^{4}}{r}\right]^{1 / 4}+K_{\min }
$$

bestimmt, wobei $r$ die Anzahl der gültigen (nichtzensurierten) Proben und $N$ die Gesamtzahl aller geprüften Proben darstellen.

Die Abhängigkeit der Bruchzähigkeit von der Probengeometrie nach der Weakest-LinkTheorie ermöglicht eine Normierung der Zähigkeitswerte auf eine einheitliche Probendicke von $25,4 \mathrm{~mm}\left(, 1 \mathrm{~T}^{“}\right)$ mit:

$$
K_{J c(x)}=K_{\min }+\left(K_{J c(0)}-K_{\min }\right) \cdot\left(\frac{B_{0}}{B_{x}}\right)^{1 / 4}
$$

wobei $K_{J c(x)}=K_{J c(1 T)}, K_{J c(0)}=K_{J c}$ für Proben mit der Dicke $B_{0}$ und $B_{x}=B_{(1 T)}=25,4 \mathrm{~mm}$ gilt. Hierbei entspricht $B$ jeweils der Bruttodicke der Probe, ohne Abzug der vorhandenen Seitkerben.

Die Bruchzähigkeit $K_{J c}$ einer Probe wird zensuriert, d.h. auf ein bestimmtes Höchstmaß begrenzt, wenn die Probe im Versuch nicht spröde versagt oder wenn ihre maximale Messkapazität $K_{J c(l i m i t)}$ nach Gleichung 3.10 überschritten wurde. In diesem Fall muss von einer zu schwachen Spannungsmehrachsigkeit vor der Rissspitze ausgegangen werden und das ermittelte $K_{J c}$ wird durch $K_{J c(l i m i t)}$ ersetzt.

$$
K_{J c(\text { limit })}=\sqrt{\frac{E \cdot b_{0} \cdot \sigma_{y s}}{M_{\text {limit }} \cdot\left(1-\nu^{2}\right)}}
$$


worin $b_{0}=\left(W-a_{0}\right)$ der Länge des Probenligaments entspricht. Die Spannung $\sigma_{y s}$ ist die Streckgrenze des Materials, die bei der Prüftemperatur des Versuchs gilt. Der Wert $M_{\text {limit }}$ entspricht einem Deformationskriterium für alle Probentypen konstant mit $M_{\text {limit }}=30$.

Aus dem Wert $K_{0}$ nach Gleichung 3.8, der einer kumulativen Versagenswahrscheinlichkeit von $63 \%$ entspricht, errechnet sich die mittlere Bruchzähigkeit bei $50 \%$ Bruchwahrscheinlichkeit nach:

$$
K_{J c(m e d)}=K_{\min }+\left(K_{0}-K_{\min }\right) \cdot[\ln (2)]^{1 / 4}
$$

Im spröd-duktilen Übergangsbereich verlaufen die experimentell bestimmten Bruchzähigkeiten $K_{J c}$ üblicherweise für bestimmte Bruchwahrscheinlichkeiten nach einer einheitlich geformten Bruchzähigkeits-Temperatur-Kurve. Die in Teilen empirisch bestimmte MasterCurve beschreibt diese Temperaturabhängigkeit der Median-Bruchzähigkeit von 1T-Proben mit:

$$
K_{J c(m e d)}=30+70 \cdot \exp \left[0,019\left(T-T_{0}\right)\right]
$$

bzw. in umgestellter Form

$$
T_{0}=T-\left(\frac{1}{0,019}\right) \cdot \ln \left[\frac{K_{J c(m e d)}-30}{70}\right]
$$

wobei $T$ die Prüftemperatur und $T_{0}$ die Referenztemperatur bei einer Median-Bruchzähigkeit von $100 \mathrm{MPa} \sqrt{\mathrm{m}}$ ist.

Gleichung 3.13 gilt, wenn die $K_{J c}$-Werte aller Proben bei gleicher Temperatur ermittelt wurden (Eintemperaturmethode). Werden die Proben bei mehreren Prüftemperaturen untersucht, erfolgt die Berechnung von $T_{0}$ durch die iterative Lösung der folgenden Gleichung (Multitemperaturmethode).

$$
\sum_{i=1}^{n} \frac{\delta_{i} \cdot \exp \left(0,019 \cdot\left(T_{i}-T_{0}\right)\right)}{11+77 \cdot \exp \left(0,019 \cdot\left(T_{i}-T_{0}\right)\right)}-\sum_{i=1}^{n} \frac{\left(K_{J c}^{i}-20\right)^{4} \cdot \exp \left(0,019 \cdot\left(T_{i}-T_{0}\right)\right)}{\left(11+77 \cdot \exp \left[0,019 \cdot\left(T_{i}-T_{0}\right)\right]\right)^{5}}=0
$$

Wie viele Proben geprüft werden müssen, damit eine nach ASTM E 1921 gültige Referenztemperatur $T_{0}$ bestimmt wird, hängt vom Abstand der Prüftemperaturen gegenüber $T_{0}$ ab. Im Idealfall liegen die Prüftemperaturen zwischen $T_{0}-14 \mathrm{~K}$ und $T_{0}+50 \mathrm{~K}$. Dann wird jede Probe mit einem Faktor $n_{1}=1 / 6$ gewichtet, nach Gleichung 3.15 die MindestWichtungssumme von 1,0 und ein gültiger $T_{0}$-Wert schon mit $r_{1}=6$ Proben erreicht.

$$
\sum_{i=1}^{3} r_{i} \cdot n_{i} \geq 1
$$

Die Proben, die bei $T \leq T_{0}-15 \mathrm{~K}$ geprüft wurden, sind mit geringerer Wichtung bei der Bestimmung von $T_{0}$ zu berücksichtigen, so dass mehr als 6 Proben geprüft werden müssen. Liegt die Prüftemperatur zwischen 15 und $35 \mathrm{~K}$ unterhalb von $T_{0}$, erhält die Probe einen Wichtungsfaktor $n_{2}=1 / 7$, bei Prüftemperaturen von 36 bis $50 \mathrm{~K}$ unterhalb von $T_{0}$ nur noch $n_{3}=1 / 8$. Die Wichtung einer Probe erfolgt mit $n=0$, wenn der gemessene $K_{J c^{-}}$ Wert die Messkapazität $K_{J c(l i m i t)}$ nach Gleichung 3.10 überschreitet (Zensorierung) oder die Prüftemperatur mehr als $50 \mathrm{~K}$ von $T_{0}$ abweicht. Der Wert $r_{i}$ in Gleichung 3.15 entspricht der Anzahl gültiger Proben in den drei genannten Temperaturbereichen.

Die Ergebnisse dieser Auswertungen nach dem Master-Curve-Konzept nach ASTM E 1921 sind ausführlich für alle untersuchten Flussstahlproben in den Abbildungen A.11 bis A.18 
dargestellt. Abbildung 3.8 zeigt eine Zusammenfassung aller ermittelten Bruchzähigkeiten in Abhängigkeit der auf die Übergangstemperatur $T_{0}$ normierten Prüftemperatur. Von den insgesamt 83 ermittelten $K_{J c(1 T)}$-Werten liegen nur $2(2,4 \%)$ unterhalb und $5(6 \%)$ oberhalb der Bruchzähigkeitskurven für $2 \%$ bzw. $98 \%$ Bruchwahrscheinlichkeit. Die zwei unterhalb liegenden Bruchzähigkeiten gehören beide zu der Flussstahlprobe PA2. Mit Ausnahme dieser Probe wird in Abbildung 3.8 deutlich, dass die Bruchzähigkeit jeder einzelnen Flussstahlprobe im unteren spröd-duktilen Übergangsbereich mit der Master-Curve recht genau beschrieben werden kann.

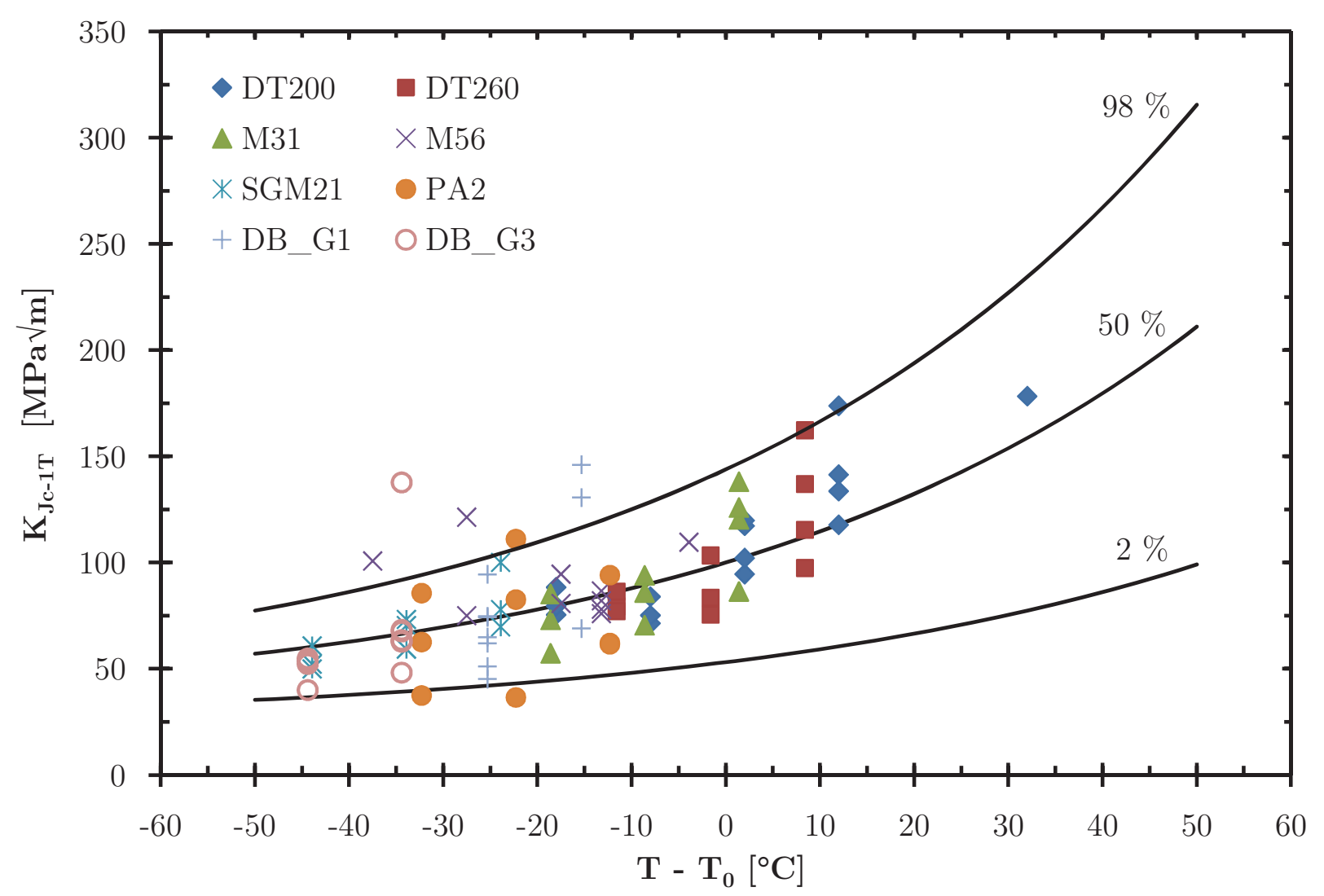

Abb. 3.8.: Bruchzähigkeit $K_{J c-1 T}$ in Abhängigkeit der auf die Übergangstemperatur $T_{0}$ der einzelnen Testserie normierten Prüftemperatur $T$

Die Prüfnorm ASTM E 1921 gilt streng genommen nur für makroskopisch homogenes Material. Inhomogenitäten führen in der Master-Curve-Auswertung üblicherweise zu überdurchschnittlich großer Datenstreuung, wie z. B. bei der bereits genannten Probe PA2. Nach ASTM E 1921 liegt eine mögliche Gefügeinhomogenität vor, wenn deutlich mehr als $4 \%$ der Datenpaare außerhalb der $2 \%$ - und $98 \%$-Streubänder liegen.

Der multimodale Master-Curve-Ansatz nach Wallin; NevasmaA et al. (2004) ermöglicht die Analyse von Datensätzen, die aus mehreren Teilpopulationen bestehen, z. B. für Datensätze aus verschiedenen Chargen eines Materials oder makroskopisch inhomogenem Material. Damit eignet sich diese Methode besonders zur gemeinsamen Auswertung der Bruchzähigkeiten aller hier untersuchten Flussstahlproben. Sie ist allerdings ungleich aufwendiger als das Standardverfahren nach ASTM E 1921.

Die multimodale MC-Methode geht davon aus, dass jede Teilpopulation, also jede Charge des 
untersuchten Materials, durch eine eigene Referenztemperatur $T_{0}$ gekennzeichnet ist. Unter der Annahme, dass diese Temperaturen einer Gaußverteilung folgen, können sie durch die Parameter $T_{0, M M}$ und die Standardabweichung $\sigma_{T_{0}, M M}$ beschrieben werden. Für die Wahrscheinlichkeitsdichtefunktion gilt damit:

$$
f_{T}=\frac{1}{\sigma_{T_{0}, M M} \cdot \sqrt{2 \pi}} \cdot \exp \left[-\frac{\left(T_{0}-T_{0, M M}\right)^{2}}{2 \cdot\left(\sigma_{T_{0}, M M}\right)^{2}}\right]
$$

Für jeden Datenpunkt einer Teilpopulation, also die Bruchzähigkeit $K_{J c}$ bei einer bestimmten Temperatur $T$, kann die „Überlebenswahrscheinlichkeit“ (kein Bruch) $S_{T_{0}}$ bei $T_{0}$ angegeben werden. Die Wahrscheinlichkeit folgt der im Master-Curve-Konzept üblichen Weibullverteilung:

$$
S_{T_{0}}=\exp \left[-\left(\frac{K_{J c}-K_{\min }}{K_{0}-K_{\min }}\right)^{4}\right]
$$

wobei $K_{\min }=20 \mathrm{MPa} \sqrt{\mathrm{m}}$ und

$$
K_{0}=31+77 \cdot \exp \left(0,019 \cdot\left(T-T_{0}\right)\right)
$$

Die zugehörige Wahrscheinlichkeitsdichte ergibt sich entsprechend zu:

$$
f_{T_{0}}=\frac{\partial S_{T_{0}}}{\partial K_{J c}}=4 \cdot \frac{\left(K_{J c}-K_{m i n}\right)^{3}}{\left(K_{0}-K_{m i n}\right)^{4}} \cdot \exp \left[-\left(\frac{K_{J c}-K_{m i n}}{K_{0}-K_{m i n}}\right)^{4}\right]
$$

Jede Teilpopulation besitzt ihre eigene $T_{0}$ und $\sigma_{T_{0}}$, so dass die „Überlebenswahrscheinlichkeit" eines Datenpunktes in der Gesamtpopulation bei einer bestimmten Temperatur und Bruchzähigkeit alle $T_{0}$ wie auch deren Dichteverteilungsfunktionen berücksichtigen muss. Dies führt zu:

$$
S=\int_{-\infty}^{\infty} f_{T} \cdot S_{T_{0}} \mathrm{~d} T_{0}
$$

Die zugehörige Gesamt-Wahrscheinlichkeitsdichte ist definiert als:

$$
f=\frac{\partial S}{\partial K_{J c}}=\int_{-\infty}^{\infty} f_{T} \cdot f_{T_{0}} \mathrm{~d} T_{0}
$$

Die Parameter $T_{0, M M}$ und $\sigma_{T_{0}, M M}$ der Gesamtpopulation werden auf Grundlage des MaximumLikelihood-Verfahrens durch Maximierung des folgenden Ausdrucks bestimmt:

$$
\ln L=\sum_{i=1}^{n}\left[\delta_{i} \cdot \ln \left(f_{i}\right)+\left(1-\delta_{i}\right) \cdot \ln \left(S_{i}\right)\right]
$$

worin $n$ der Anzahl der Datenpunkte entspricht und $\delta_{i}$ den bekannten Zensurierungsparameter darstellt, welcher dem Wert 1 bei gültigen Datenpunkten und dem Wert 0 bei ungültigen entspricht. Übersteigt für einen Datenpunkt die Bruchzähigkeit die Messkapazität $K_{J c(\text { Limit) }}$ der Probe (Gleichung 3.10), ist auch hier der Bruchzähigkeitswert durch $K_{J c(\text { limit) }}$ zu ersetzen. 
Mit Hilfe der auf diesem Weg bestimmten Werte $T_{0, M M}$ und $\sigma_{T_{0}, M M}$ kann die Versagenswahrscheinlichkeit

$$
1-S=1-\int_{-\infty}^{\infty} f_{T} \cdot S_{T_{0}} \mathrm{~d} T_{0}
$$

der gesamten Population abgeleitet werden. Dazu wird $K_{J c}$ für jede Temperatur im Bereich $T_{0, M M}-2 \cdot \sigma_{T_{0}, M M}-50{ }^{\circ} \mathrm{C}$ bis $T_{0, M M}+2 \cdot \sigma_{T_{0}, M M}+50{ }^{\circ} \mathrm{C}$ ermittelt.

Die Ergebnisse dieser Auswertung für die hier untersuchten Flussstähle sind in Abbildung 3.9 dargestellt und der Standard-Master-Curve nach ASTM E 1921 gegenübergestellt. Erwartungsgemäß werden die Bruchzähigkeiten der inhomogenen Gesamtpopulation „Flussstahl“ durch die multimodale Master-Curve besser erfasst. Darüber hinaus fällt aber auf, dass mit Ausnahme der Probe PA2 die Bruchzähigkeit der Flussstähle auch von der Standard-MasterCurve ausreichend genau beschrieben wird.

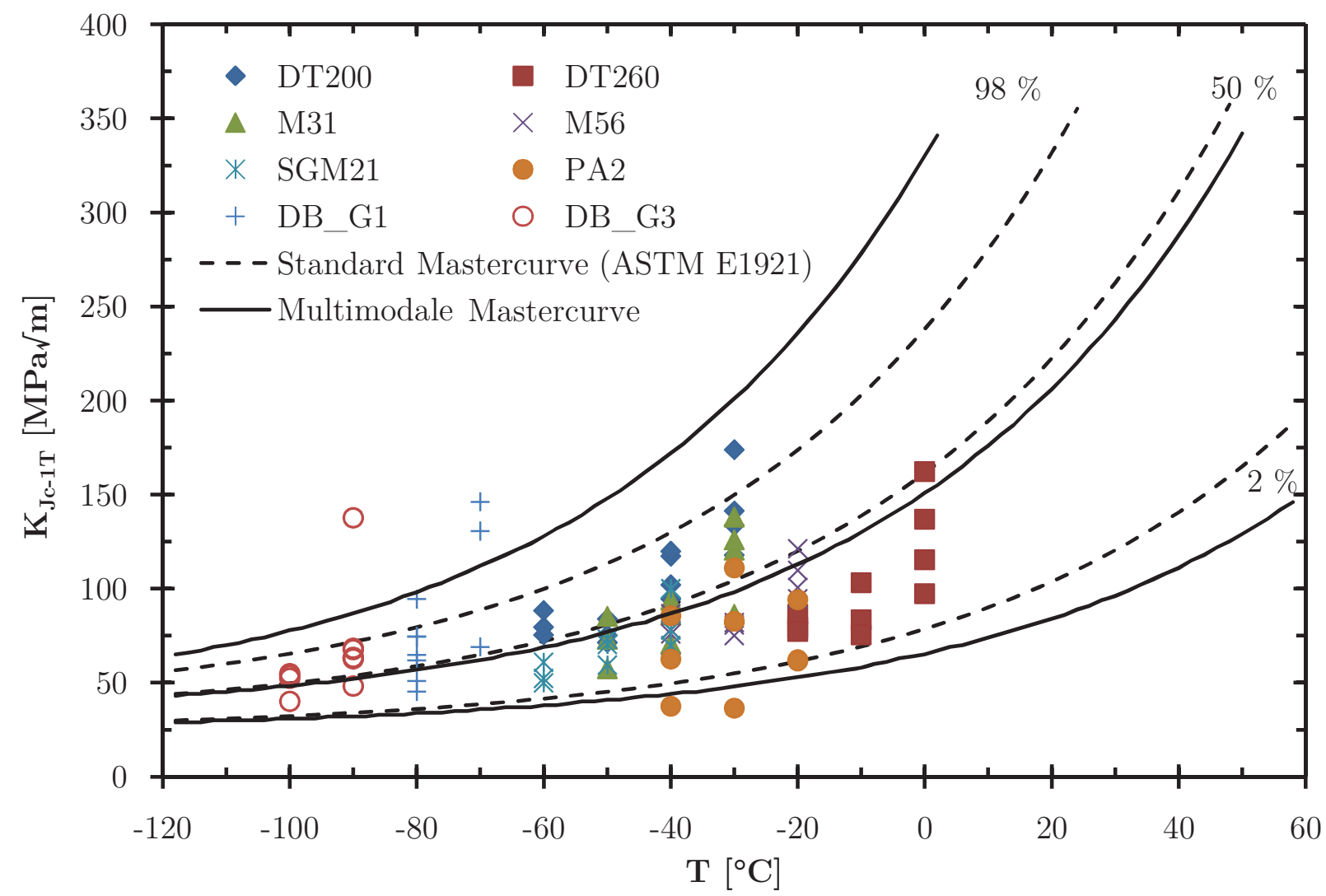

Abb. 3.9.: Auswertung der $K_{J c-1 T}$-Werte aller Testserien nach ASTM E 1921:2009 und dem multimodalen Master-Curve Auswerteverfahren für inhomogene Stähle

Als Grundlage für die bruchmechanische Sicherheitsanalyse gelochter Bauteile in Kapitel 5 ergeben sich analog zu Gleichung 3.12 die 5\%-Fraktilkurven für die Standard-Master-Curve $\mathrm{zu}$ :

$$
K_{J c-1 T, 5 \%}=25,2+36,6 \cdot \exp (0,019 \cdot(T+33,3))
$$

und für die multimodale Master-Curve zu:

$$
K_{J c-1 T, 5 \%}^{M M}=25,9+29,7 \cdot \exp (0,0186 \cdot(T+30,0))
$$




\subsubsection{Vergleich mit Bruchzähigkeiten alter Brückenbaustähle}

Bereits seit den 1980er Jahren werden bruchmechanische Untersuchungen an alten Baustählen durchgeführt, mit dem Ziel, die Sprödbruchsicherheit insbesondere von bestehenden Stahlbrückenkonstruktionen zu bewerten. Um die im Rahmen dieser Arbeit experimentell ermittelten Bruchzähigkeiten alter Flussstähle zu validieren, werden diese den Ergebnissen früherer Untersuchungen gegenübergestellt.

Neben Arbeiten von Brühwiler; Hirt et al. (1989), Wittemann; Baehre (1993) und MANG; BUCAK (1996) wurden insbesondere am Institut für Eisenhüttenkunde der RWTH Aachen Untersuchungen an alten Brückenbaustählen durchgeführt. Die dabei gewonnenen Ergebnisse wurden u.a. von Hensen (1992), Niessen (1994), Langenberg (1996) und StötzeL (1998) ausgewertet und in unterschiedlichen Konzepten zum Nachweis der Sprödbruchsicherheit genieteter Bauteile verwendet. Auch in internationalen Arbeiten, wie z. B. NCHRP-302 (1987), LARsson (2009), Cremona; Patron et al. (2007) und Moreno; VALIENTE (2007) wurden Ergebnisse bruchmechanischer Untersuchungen an alten Baustählen veröffentlicht. Während die Herkunft der untersuchten Werkstoffe i. d. R. angegeben wurde, sind in nahezu allen genannten Quellen die Art der Versuchsdurchführung oder die zugrunde liegende Werkstoffprüfnorm, die Probengeometrien und vereinzelt auch die Prüftemperatur nur unzureichend dokumentiert. Lediglich bei LANGENBERG (1996) sind Bruchzähigkeiten alter Flussstähle einschließlich der Randbedingungen zu ihrer Bestimmung angegeben.

Die Ermittlung der Bruchzähigkeit erfolgte nach ASTM E 813:1989 an 0,5T-C(T)-Proben, wobei neben normgerechten Proben mit 12,5 mm Dicke auch untermaßige mit bis $\mathrm{zu} 7 \mathrm{~mm}$ Dicke untersucht wurden. Die ermittelten Bruchzähigkeiten konnten mit Hilfe von Gleichung 3.9 auf die Referenzdicke von "1T" umgerechnet werden, um sie in Abbildung 3.10 der in dieser Arbeit abgeleiteten Master-Curve gegenüber zu stellen. Auch die Bruchzähigkeit der von LANGENBERG (1996) untersuchten Brückenbaustähle wird mit der multimodalen Master-Curve, bis auf wenige Ausnahmen, gut beschrieben. Es fällt jedoch auf, dass mit steigender Temperatur die Bruchzähigkeit $K_{J c}$ im Mittel zu sinken scheint. Dies ist in der Auswertung bzw. der Interpretation der Versuchsergebnisse begründet.

In Abhängigkeit des Bruchverhaltens der Proben wurde zwischen sprödem Versagen (Spaltbruch) und stabiler Rissinitiierung unterschieden. Versagte die Probe komplett spröde ohne sichtbar duktile Rissanteile auf der Bruchfläche, so wurde die ermittelte Bruchzähigkeit mit $J_{c}$ bezeichnet. Die äquivalenten $K_{J c}$-Werte sind in Abbildung 3.10 dargestellt. Trat vor dem Spaltbruchversagen ein duktiles und auf der Bruchfläche sichtbares Risswachstum auf, wurde die Bruchzähigkeit mit $J_{i}$ bezeichnet. Da der Umfang duktilen Risswachstums nicht dokumentiert wurde, sind diese Werte in der Gegenüberstellung nicht enthalten.

Die in dieser Arbeit angewendete Versuchsdurchführung und -auswertung nach ASTM E 1921 quantifiziert ebenfalls die Bruchzähigkeit bei sprödem Versagen. Die Norm lässt jedoch vor Eintreten des Spaltbruchs ein geringes Maß an duktilem Risswachstum bis zu einer Rissverlängerung von $0,05\left(W-a_{0}\right)<1 \mathrm{~mm}$ zu. Darüber hinaus wird die Bruchzähigkeit auf die maximale Messkapazität $K_{J c(l i m i t)}$ der Probe nach Gleichung 3.10 begrenzt. Die mit der Master-Curve beschriebene Bruchzähigkeit steigt folglich erwartungsgemäß mit höheren Temperaturen an.

Um die ermittelte Bruchzähigkeit alter Flussstähle des Stahlhochbaus mit den besseren Flussstahlgüten vergleichen zu können, wurden zusätzliche Untersuchungen an Werkstoffproben 
aus einer geschweißten Stahlfachwerk-Konstruktion (Baujahr 1937) durchgeführt. Neben einer Probe aus Profilstahl wurde insbesondere das Material aus drei Grobblechen analysiert. Die Ergebnisse der chemischen Analysen sind in Tabelle 3.5 zusammengefasst. Anhand des Kohlenstoff-, Phosphor- und Stickstoffgehalts können alle vier Proben eindeutig als im Herdofen hergestellte Siemens-Martin-Stähle identifiziert werden. Im Unterschied zu allen anderen untersuchten Werkstoffen enthält die Probe B3 etwa 0,2 \% Silizium und ist damit ein vollberuhigt vergossener Stahl [REICHE (2000)]. Die an Rundzugproben B5 nach DIN 50125:2009 ermittelten Festigkeitskennwerte sind in Tabelle 3.6 zusammengefasst.

Zur Quantifizierung der Werkstoffzähigkeit der vier Siemens-Martin-Stähle wurden, analog zu den Werkstoffchargen des Stahlhochbaus, umfangreiche Kerbschlagbiegeversuche nach DIN EN ISO 148-1:2011 und bruchmechanische Untersuchungen mit Auswertung nach dem Master-Curve-Konzept in ASTM E 1921:2009 durchgeführt. Aufgrund des nur in geringer Menge zur Verfügung stehenden Materials der Profilstahlprobe B1 konnten von diesem Werkstoff nur drei C(T)-Proben gefertigt und untersucht werden. Die Ergebnisse der Analysen der drei Werkstoffproben B2 bis B4 aus Grobblech sind in Anhang A dargestellt. Erwartungsgemäß liegt die Übergangstemperatur $T_{27 J}$ der Kerbschlagarbeit, wie auch die Referenztemperatur $T_{0}$ der Bruchzähigkeit, für den beruhigt vergossenen Stahl B3 deutlich niedriger als bei den anderen beiden Blechproben. Allgemein betrachtet, weisen die untersuchten Siemens-Martin-Stähle deutlich tiefere Referenztemperaturen auf als die untersuchten Stähle des Hochbaus. Dies wird auch in der Gegenüberstellung der Bruchzähigkeiten mit der in Abschnitt 3.4 ermittelten Master-Curve in Abbildung 3.11 deutlich.

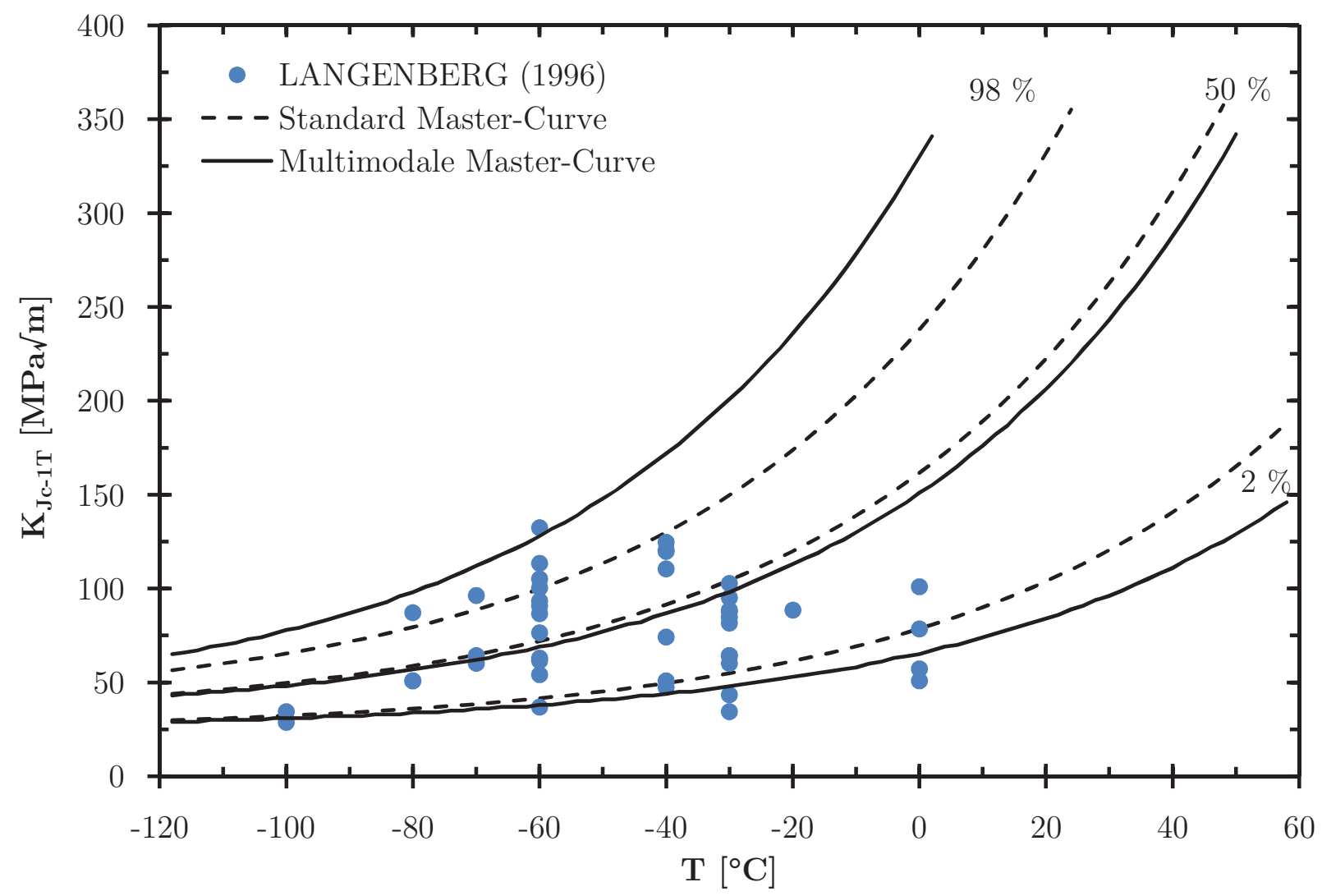

Abb. 3.10.: Gegenüberstellung existierender $K_{J c-1 T}$-Werte von Flussstählen aus Brückenbauwerken mit den Ergebnissen der Master-Curve-Analyse 
Tab. 3.5.: Ergebnisse der chemischen Analysen an den Bauteilen einer geschweißten Konstruktion aus Flussstahl, Referenzwerte nach REICHE (2000)

\begin{tabular}{l|c|c|c|c|c|c|c|c}
\hline \multirow{2}{*}{ Probe } & \multicolumn{8}{|c}{ Chemische Analyse [\%] } \\
& $\mathrm{C}$ & $\mathrm{Si}$ & $\mathrm{Mn}$ & $\mathrm{P}$ & $\mathrm{S}$ & $\mathrm{Al}$ & $\mathrm{Cu}$ & $\mathrm{N}$ \\
\hline B1 (L80x8) & 0,070 & 0,000 & 0,350 & 0,029 & 0,028 & 0,000 & 0,147 & 0,0047 \\
B2 (Bl13) & 0,140 & 0,007 & 0,420 & 0,038 & 0,044 & 0,000 & 0,052 & 0,0057 \\
B3 (Bl13) & 0,090 & 0,202 & 0,420 & 0,018 & 0,027 & 0,002 & 0,113 & 0,0053 \\
B4 (B111) & 0,190 & 0,011 & 0,410 & 0,065 & 0,030 & 0,001 & 0,068 & 0,0074 \\
\hline Thomas-Stahl & 0,048 & 0,009 & 0,46 & 0,051 & 0,044 & - & - & 0,0140 \\
Siemens-Martin-Stahl & 0,090 & 0,008 & 0,480 & 0,035 & 0,038 & - & - & 0,0050 \\
\hline
\end{tabular}

Tab. 3.6.: Mittlere Festigkeitskennwerte bei RT der Bauteile einer geschweißten Konstruktion aus Flussstahl

\begin{tabular}{l|c|c|c}
\hline Probe & $R_{e l}\left[\mathrm{~N} / \mathrm{mm}^{2}\right]$ & $R_{m}\left[\mathrm{~N} / \mathrm{mm}^{2}\right]$ & $A[\%]$ \\
\hline B1 & 237,1 & 341,5 & 41,4 \\
B2 & 288,2 & 382,4 & 34,4 \\
B3 & 307,5 & 395,5 & 34,9 \\
B4 & 316,9 & 449,0 & 31,6 \\
\hline Mittelwert nach ReICHE (2000) & 268 & 393 & 34,8 \\
Mittelwert nach LaNGENBERG (1996) & 282 & 417 & 27,0 \\
\hline
\end{tabular}

Von den acht untersuchten Flussstählen des Stahlhochbaus können die beiden Winkelprofile M31 und M56 (siehe Tabelle 3.2) ebenfalls als Siemens-Martin-Stahl klassifiziert werden. Wertet man die Bruchzähigkeiten dieser beiden Werkstoffe zusammen mit denen der drei unberuhigt vergossenen Flussstähle B1, B2 und B4 nach ASTM E 1921 aus, ergibt sich eine Referenztemperatur $T_{0}$ von ca. $-45^{\circ} \mathrm{C}$ (siehe Abbildung 3.12). Diese liegt damit um etwa 12 bis $15^{\circ} \mathrm{C}$ tiefer als für allgemeine Flussstähle nach Abschnitt 3.4. Analog zu Gleichung 3.24 ergibt sich für Siemens-Martin-Stähle die 5\%-Fraktilkurve der Standard-Master-Curve zu:

$$
K_{J c-1 T, 5 \%}=25,2+36,6 \cdot \exp (0,019 \cdot(T+45,2))
$$

\subsubsection{Korrelationen der Werkstoffzähigkeit}

Da für die Beurteilung der Sprödbruchsicherheit bestehender wie auch neuer Bauteile und Bauwerke eine direkte Messung der Bruchzähigkeit des verwendeten Werkstoffs häufig nicht möglich ist oder aufgrund der Komplexität der Untersuchungen vermieden werden soll, wurden bereits zahlreiche Forschungsarbeiten mit dem Ziel durchgeführt, Korrelationen der Bruchzähigkeit zu anderen Werkstoffeigenschaften abzuleiten. Die meisten Untersuchungen konzentrierten sich dabei auf eine Abschätzung von $K_{I c}$ aus der Kerbschlagarbeit $K V$ im Kerbschlagbiegeversuch. Umfangreiche Zusammenstellungen solcher Korrelationsbeziehungen sind bei PhaAl; Macdonald (1991), Michels; Hagedorn (1997), BAnnister (1998) 


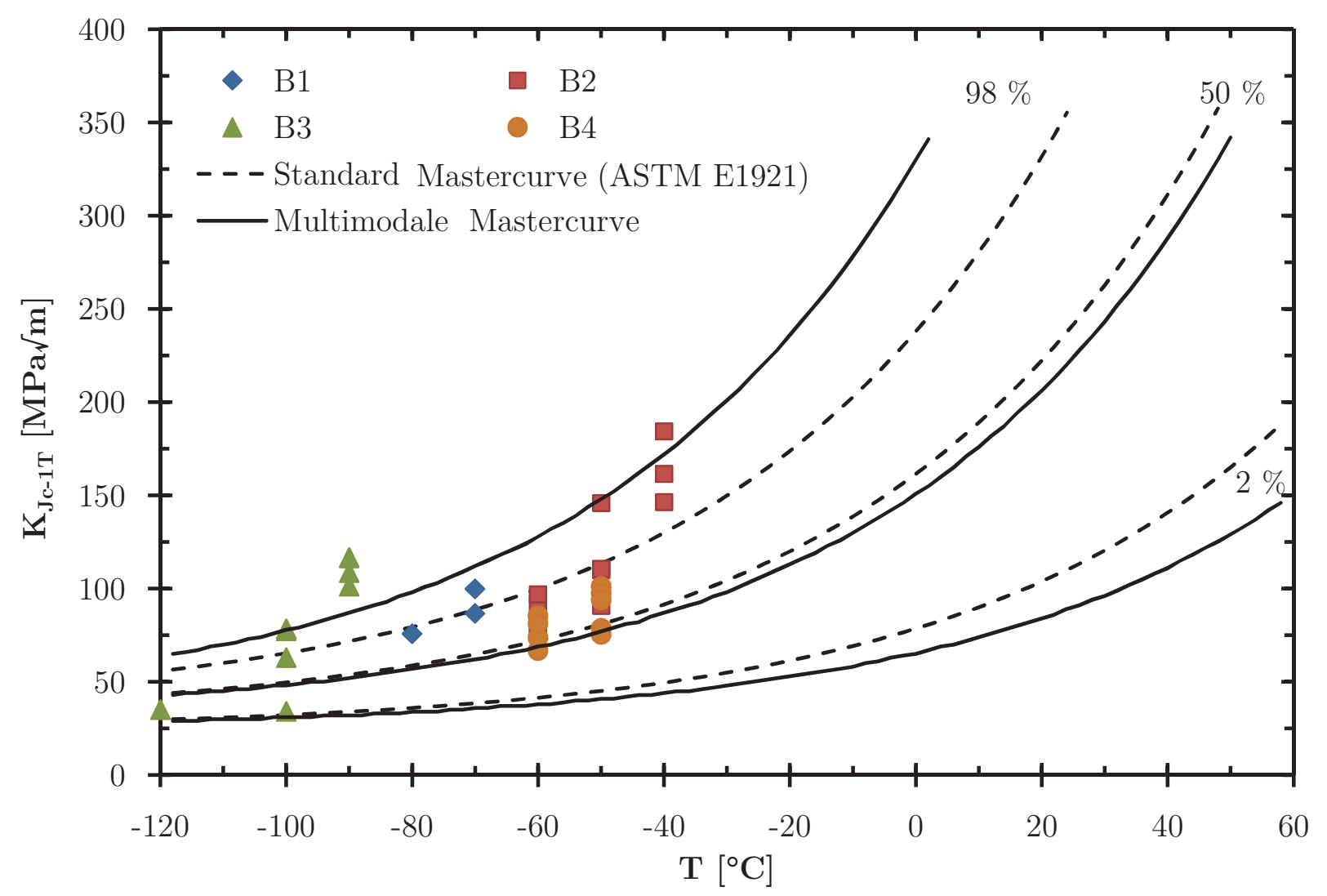

Abb. 3.11.: Gegenüberstellung der Bruchzähigkeit von Flussstählen einer geschweißten Konstruktion mit den Ergebnissen der Master-Curve-Analyse

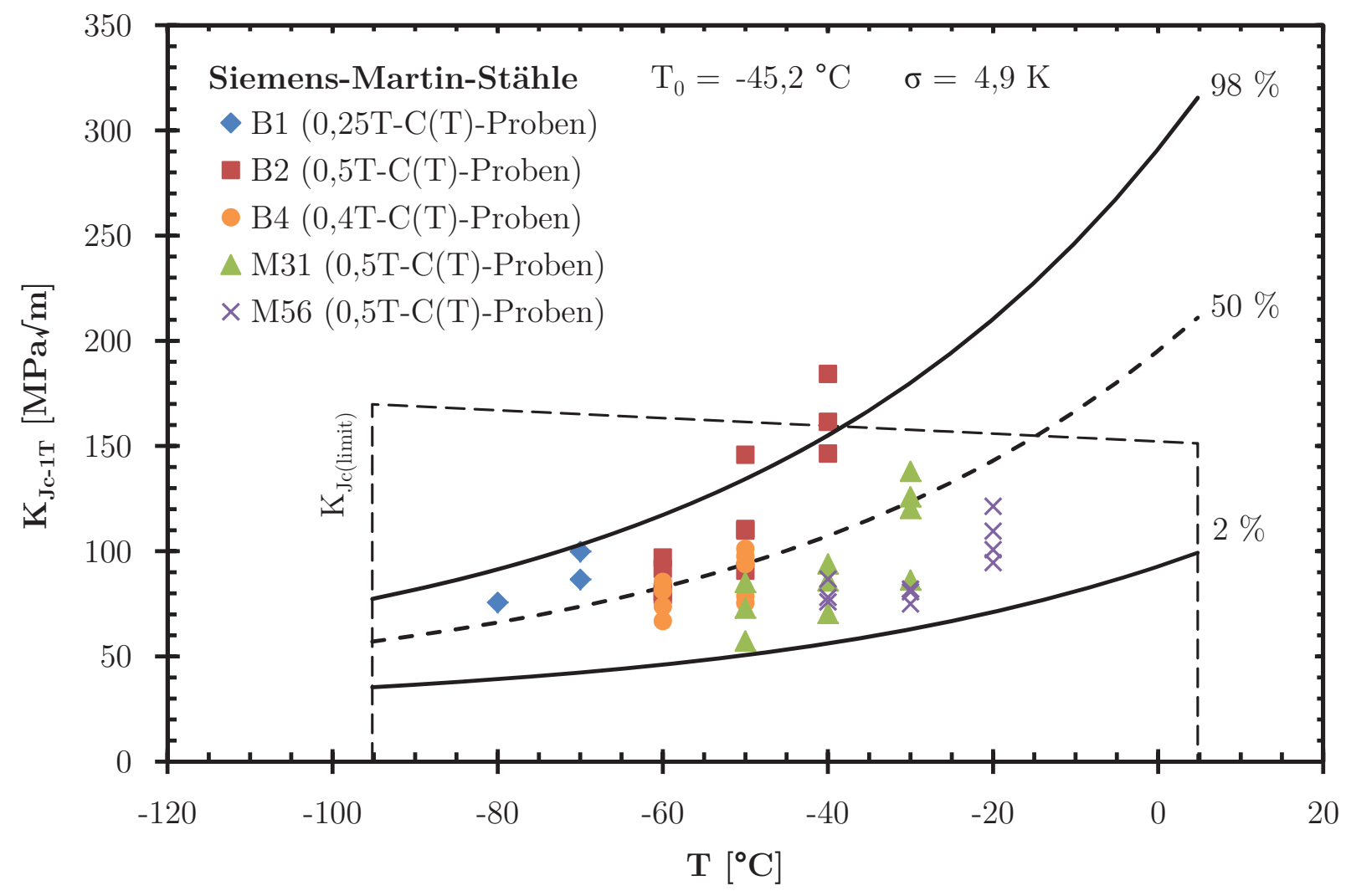

Abb. 3.12.: Auswertung der $K_{J c-1 T}$-Werte aller Siemens-Martin-Stähle nach ASTM E 1921 
und Meyendorf (1999) dokumentiert und kritisch diskutiert. Darüber hinaus wurden von Michels (1995) und Müller; Pusch (2001) weitere Korrelationen der Risszähigkeit mit den zusätzlichen Versuchsdaten des instrumentierten Kerbschlagbiegeversuchs, z. B. der Rissauffangkraft $F_{a}$ (siehe Abbildung 3.4), abgeleitet.

Generell gelten diese rein empirisch ermittelten Beziehungen nur für bestimmte Werkstoffe oder Werkstoffzustände und sagen entweder einen Mittelwert der Risszähigkeit oder einen unteren Grenzwert voraus. Die Anwendung solcher Korrelationen ist deshalb im Allgemeinen als schwierig zu beurteilen bzw. ihre Eignung ist kritisch zu prüfen. Aus diesem Grund werden im Folgenden die bei SINTAP [BAnnister; Webster (1999)] und in der FKM-Richtlinie „Bruchmechanischer Festigkeitsnachweis für Maschinenbauteile“ [BERger; Blauel et al. (2006)] empfohlenen Abschätzungen der Bruchzähigkeit aus den Ergebnissen des Kerbschlagbiegeversuchs hinsichtlich ihrer Eignung für alte Flussstähle überprüft.

Die Abschätzung eines unteren Grenzwertes der Risszähigkeit $K_{m a t}$ im spröden Werkstoffzustand wird nach der Beziehung

$$
K_{\text {mat }}=(12 \cdot \sqrt{K V}-20) \cdot\left(\frac{25}{B}\right)^{0,25}+20
$$

in $\mathrm{MPa} \sqrt{\mathrm{m}}$ empfohlen. Bei der in den Versuchen ermittelten, minimalen Kerbschlagarbeit $K V_{2}$ von $4,5 \mathrm{~J}$ und einem Bezugswert $K_{I c}$ einer $1 \mathrm{~T}-\mathrm{C}(\mathrm{T})$-Probe, ergibt sich eine Mindestrisszähigkeit $K_{\text {mat }}$ von $25,5 \mathrm{MPa} \sqrt{\mathrm{m}}$. Dieser Mindestwert liegt unterhalb aller ermittelten Bruchzähigkeiten $K_{J c-1 T}$ und stellt damit auch für die hier untersuchten Flussstähle eine geeignete, konservative Abschätzung dar.

Die Risszähigkeit im unteren Übergangsbereich der Risszähigkeits-Temperatur-Kurve kann als eine Funktion der Übergangstemperatur $T_{27 J}$ abhängig von der Temperatur $T$ und für eine bestimmte Versagenswahrscheinlichkeit $P_{f}$ mit

$$
K_{\text {mat }}=20+\left(11+77 \cdot e^{0,019 \cdot\left(T-T_{27 J}+3\right)}\right) \cdot\left(\frac{25}{B}\right)^{0,25} \cdot\left(\ln \frac{1}{1-P_{f}}\right)^{0,25}
$$

in $\mathrm{MPa} \sqrt{\mathrm{m}}$ abgeschätzt werden. Wie in Abbildung 3.13 ersichtlich, ist auch dieser Ansatz nach FKM-Richtlinie für die hier untersuchten Flussstähle anwendbar, führt aber erwartungsgemäß zu einer sehr konservativen Abschätzung der Risszähigkeits-Temperaturkurve.

Zur Abschätzung der Übergangstemperatur $T_{27}$, durch Extrapolation aus Kerbschlagarbeiten, die bei einer anderen Temperatur bestimmt worden sind, bietet die Richtlinie in einem Temperaturbereich von $\pm 30^{\circ} \mathrm{C}$ eine tabellierte Übergangskurve, die in Abbildung 3.14 den Ergebnissen der hier durchgeführten Untersuchungen gegenüber gestellt ist. Die bereits in der FKM-Richtlinie angeführte Warnung, dass kohlenstoffarme Stähle eine meist steilere Übergangskurve aufweisen, trifft auch auf nahezu alle untersuchten Flussstähle zu. Folglich wäre eine Abschätzung von $T_{27 J}$ aus höheren Temperaturen nicht mehr konservativ. Da die Übergangstemperatur $T_{27 J}$ alter Flussstähle meist bei Raumtemperatur oder höher liegt und Kerbschlagbiegeversuche in der Praxis häufig bei tieferen Temperaturen durchgeführt werden, ist eine konservative Abschätzung von $T_{27 J}$ aus Temperaturen unterhalb $T_{27 J}$ dennoch möglich.

Die im Stahlbau anerkannte Beziehung zwischen Kerbschlagarbeit und Bruchzähigkeit ist die von SANZ (1980) entwickelte Korrelation der Übergangstemperaturen $T_{27 J}$ und $T_{100}$, die in einer modifizierten Form auch Bestandteil der Stahlgütewahl nach DIN EN 1993-1-10:2010 


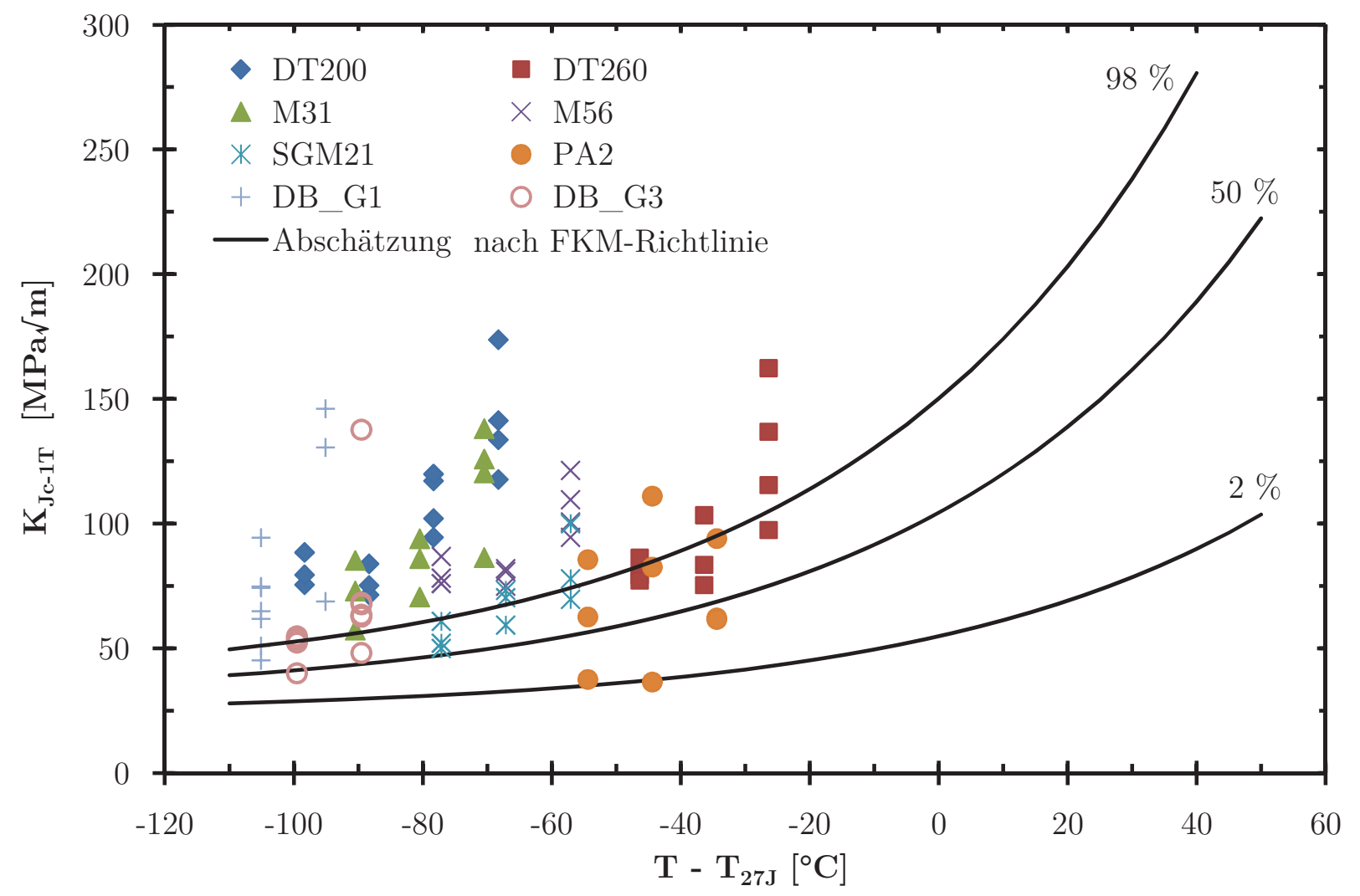

Abb. 3.13.: Abschätzung der Risszähigkeits-Temperatur-Kurve in Abhängigkeit der Übergangstemperatur $T_{27 J}$ nach FKM-Richtlinie [BERGER; BLAUEL et al. (2006)]

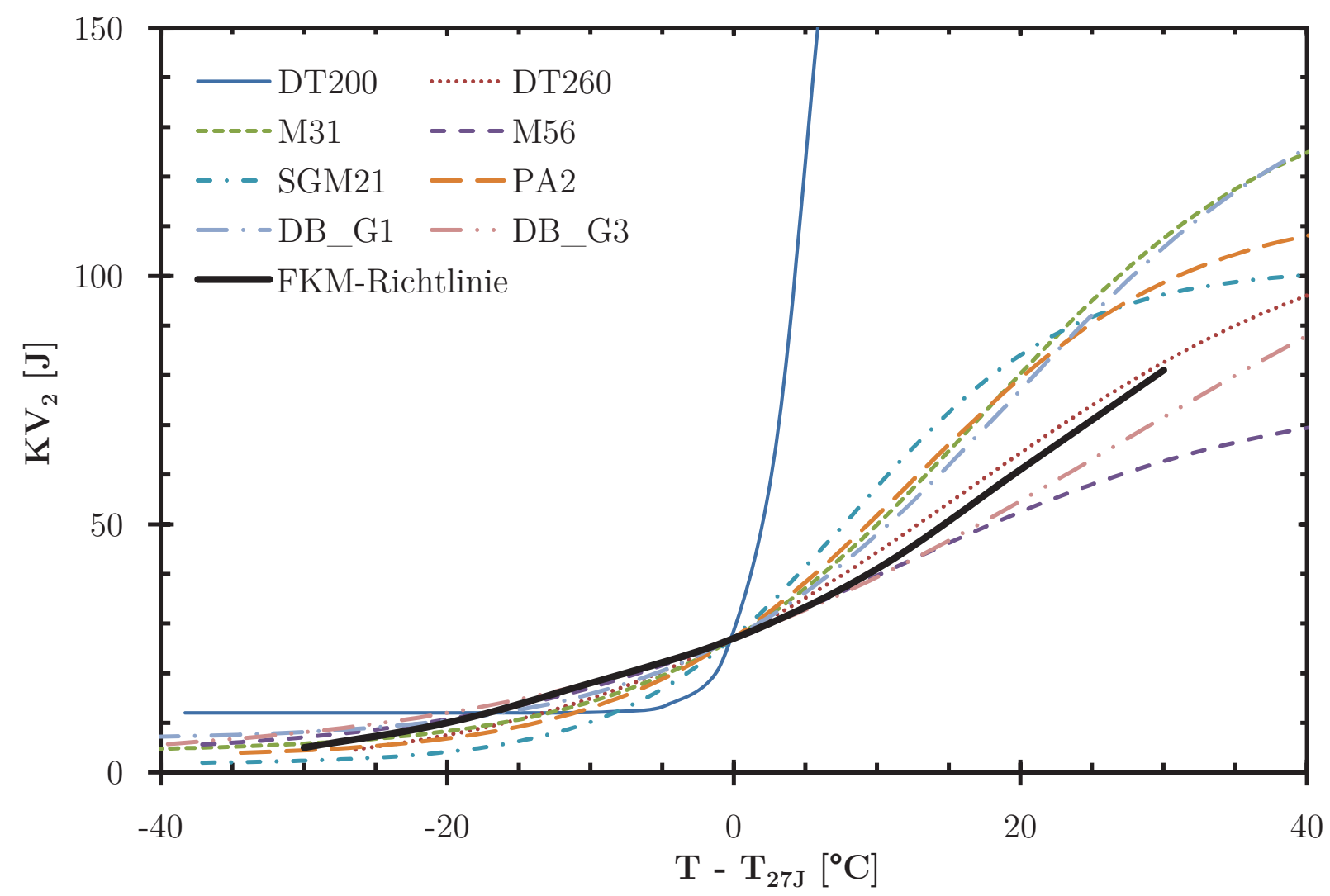

Abb. 3.14.: Kerbschlag-Temperaturkurven bezogen auf die Übergangstemperatur $T_{27 J}$ im Vergleich zur Übergangskurve nach FKM-Richtlinie [BERGER; BlAUEL et al. (2006)] 
ist (siehe Abschnitt 2.5). Vergleicht man die Zähigkeiten der analysierten Flussstähle mit denjenigen heutiger Baustähle (siehe Abbildung 3.15), ist deutlich zu erkennen, dass die im Eurocode verwendete Korrelationsbeziehung

$$
T_{0}=T_{27 J}-18^{\circ} \mathrm{C} \quad( \pm 2 \cdot \sigma) \quad \text { mit } \sigma=13^{\circ} \mathrm{C}
$$

für alte Baustähle nicht zutrifft. Bei vergleichbaren Referenztemperaturen $T_{0}$ der Bruchzähigkeit liegt der Übergangsbereich im Kerbschlagbiegeversuch für alte Flussstähle bei deutlich höheren Temperaturen. Dies gilt insbesondere für die unberuhigt vergossenen Stähle. Für die untersuchten Flussstähle ergibt sich gegenüber Gleichung 3.29 eine mittlere Verschiebung der Übergangstemperaturen von etwa $56^{\circ} \mathrm{C}$ bei einer Standardabweichung von etwa $19^{\circ} \mathrm{C}$.

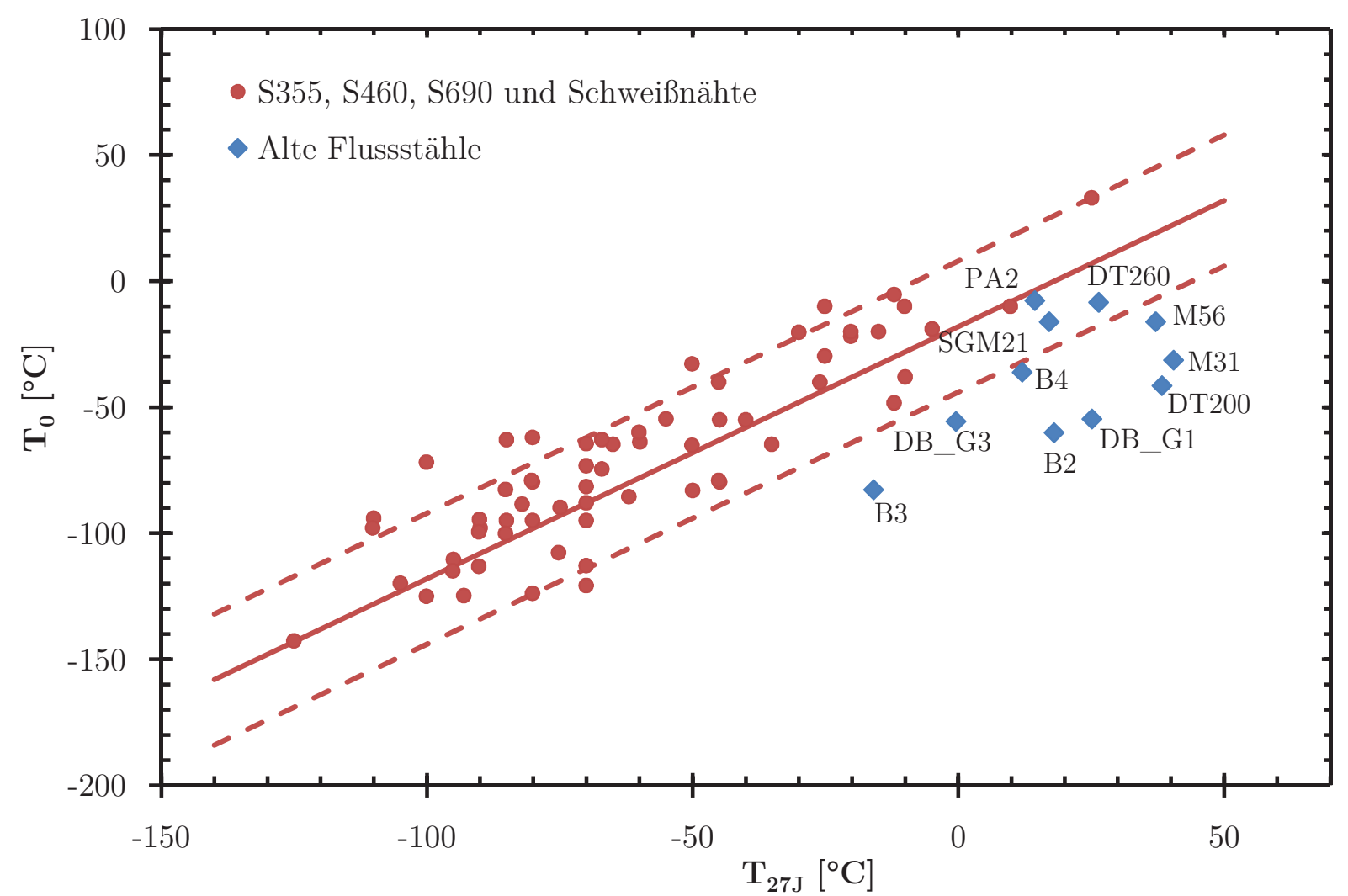

Abb. 3.15.: Gegenüberstellung der Zähigkeiten untersuchter Flussstähle mit denen heutiger Baustähle (gemäß der mod. SANZ-Korrelation in DIN EN 1993-1-10:2010)

Betrachtet man die beiden Übergangstemperaturen $T_{27 J}$ und $T_{0}$ als abhängige Zufallsgrößen, so gilt nach SPAETHE (1992) der Korrelationskoeffizient $\rho_{X Y}$ nach Gleichung 3.30 als ein Maß für die lineare Abhängigkeit. Er wird üblicherweise verwendet, um die Güte einer Korrelationsbeziehung zwischen zwei Größen zu bewerten.

$$
\begin{aligned}
\rho_{X Y} & =\frac{\operatorname{COV}(\underline{X}, \underline{Y})}{\sigma_{x} \cdot \sigma_{y}} \\
\text { Kovarianz } \operatorname{COV}(\underline{X}, \underline{Y}) & =\frac{1}{n} \sum_{i=1}^{n}\left[\left(\underline{x}_{i}-\mu_{x}\right) \cdot\left(\underline{y}_{i}-\mu_{y}\right)\right]
\end{aligned}
$$




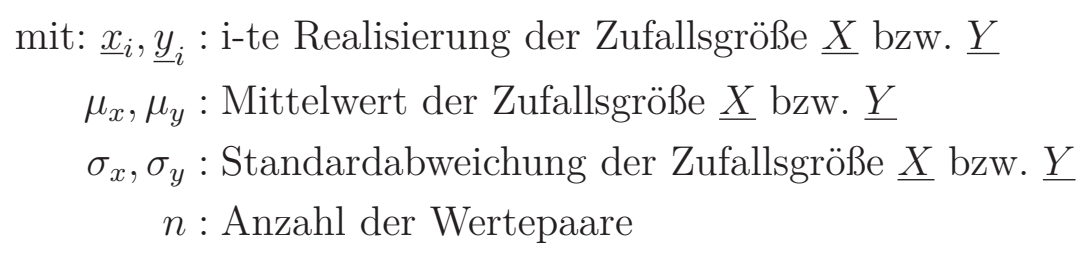

Der Koeffizient $\rho_{X Y}$ kann Werte im Intervall $-1 \leq \rho_{X Y} \leq+1$ annehmen. Im Fall $\rho_{X Y}=0$ gelten die beiden Zufallsgrößen als unkorreliert. Für $\rho_{X Y}=-1$ oder $\rho_{X Y}=+1$ sind beide Größen streng linear abhängig.

Für die der modifizierten Sanz-Korrelation zugrunde liegenden Werkstoffdaten ergibt sich nach Gleichung 3.30 ein Korrelationskoeffizient von $\rho_{X Y}=0,88$. Die Korrelation der Übergangstemperaturen nach Gleichung 3.29 trifft für heutige Baustähle folglich sehr gut zu. Eine ähnlich strenge Korrelation für alte Flussstähle ist anhand der in dieser Arbeit ermittelten Daten nicht ableitbar. Der Korrelationskoeffizient beträgt hierfür nur $\rho_{X Y}=0,56$.

\subsection{Werkstoffeigenschaften an Loch- und Bauteilrändern}

Wie bereits in Abschnitt 2.4.3 ausführlich erläutert, ist die Sprödbruchneigung gelochter, also genieteter oder geschraubter Stahlkonstruktionen ganz wesentlich von den Werkstoffeigenschaften an den Lochrändern und damit von der Herstellung dieser Löcher abhängig. Insbesondere im Stahlhochbau war das Stanzen der Löcher stark verbreitet. Die dabei verursachte Kaltverformung, in Verbindung mit verstärkter Alterungsneigung windgefrischter Flussstähle, führt zu einer Verfestigung der Lochränder und damit zu einer erhöhten Sprödbruchneigung in diesen Bereichen.

Zur Bestätigung, wie vor allem zur Quantifizierung dieser Werkstoffbeeinflussung, wurden umfangreiche Gefügeuntersuchungen durchgeführt. Ziel war es einerseits, Gefügeunterschiede zwischen den verformten Rändern und dem unverformtem Grundmaterial sichtbar zu machen. Darüber hinaus sollte die Verfestigung mit Hilfe von Härtemessungen nachgewiesen und die Ausdehnung der durch Stanzung beeinflussten Zone anhand von Härte-Tiefen-Verläufen ermittelt werden. Die Breite der verfestigten Randzone bildet eine wesentliche Grundlage für die in Abschnitt 5.3 aufgestellten Bemessungsmodelle sowie die in Kapitel 4 durchgeführten numerischen Berechnungen gelochter Bauteile.

\subsubsection{Auswahl des Probenmaterials}

Aus dem zur Verfügung stehenden Probenmaterial (Flussstähle aus den Baujahren 1897 bis 1930) erfolgte zunächst mittels chemischer Analysen eine Auswahl von 26 Proben mit Neigung zur Reckalterung. Dazu gehörten u. a. auch Bauteile, die zur Ermittlung der Bruchzähigkeit in Abschnitt 3.4 oder für die Bauteilzugversuche in Abschnitt 3.6 verwendet wurden. Da anhand des äußeren Befundes der Proben die Herstellungsart der Löcher häufig nicht sicher ermittelt werden konnte und die aufwendige Herstellung von eingebetteten Mikroschliffen nur auf geeignete Proben begrenzt werden sollte, erfolgte eine Vorauswahl an 
Makroschliffen. Diese wurden einer Makroätzbehandlung in wässriger Ammoniumpersulfatlösung unterzogen, um den Verlauf von Seigerungszeilen in Probenmitte sichtbar zu machen (siehe Abbildung 3.16). Auf diesem Weg gelang die Auswahl von insgesamt 15 Proben mit Dicken von 4 bis $12 \mathrm{~mm}$, an denen gestanzte Löcher und teilweise auch scherengeschnittene Profilenden nachgewiesen werden konnten. Damit stand Probenmaterial mit insgesamt 22 kaltverformten Werkstoffbereichen für Gefügeuntersuchungen und Härteverlaufsmessungen zur Verfügung.

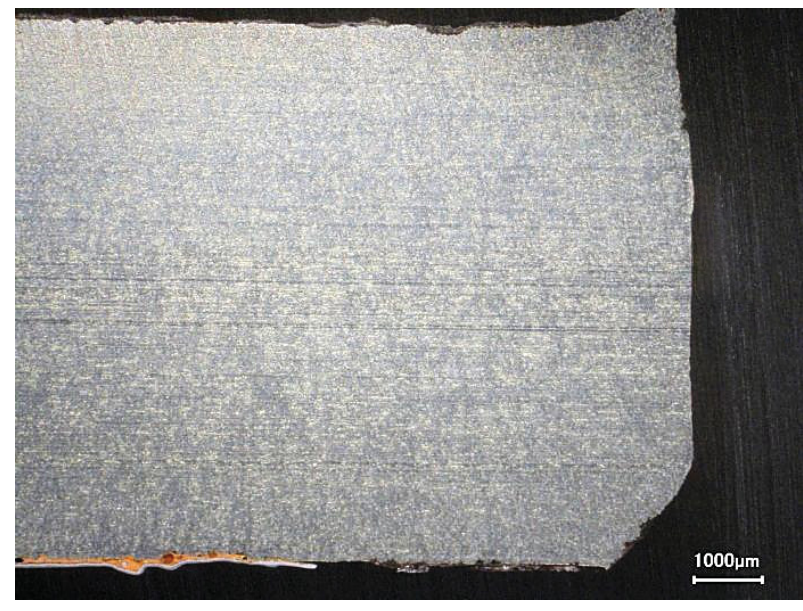

a) ungeeignet: geradlinige Seigerungszeilen am gebohrten und gefasten Lochrand

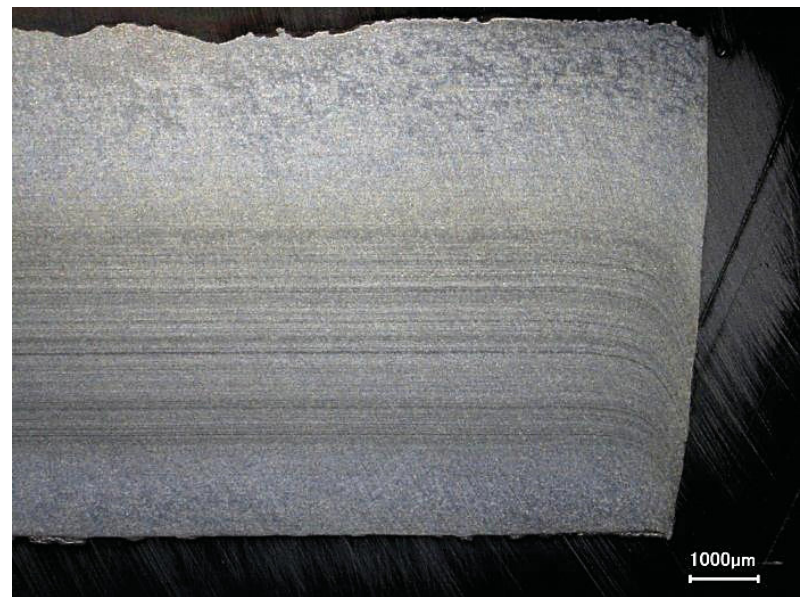

b) geeignet: verformte Seigerungszeilen am gestanzten Lochrand

Abb. 3.16.: Auswahl geeigneter Proben für Härteverlaufsmessungen an Gefügeaufnahmen nach Makroätzung in wässriger Ammoniumpersulfat-Lösung

\subsubsection{Gefügeuntersuchungen}

Alle untersuchten Werkstoffproben zeigten im Mikroschliff, nach Ätzung in alkoholischer $\mathrm{HNO}_{3}$-Lösung (Nitalätzung), das für Flussstähle typische ferritisch-perlitische Grundgefüge. Angesichts der häufig sehr niedrigen Kohlenstoffgehalte ist der Perlitanteil eher gering, seigerungsbedingt in den Speckschichten kaum mehr nachweisbar. Bei höherer optischer Vergrößerung sind die ebenso typischen und durch den Walzprozess gestreckten MangansulfidTeilchen zu erkennen. Eine gelegentlich vorhandene Strukturierung in den sonst sehr hell dargestellten Ferritkörnern deutet auf fein verteilte Eisennitritnadeln aufgrund erhöhter Stickstoffkonzentration hin (siehe z. B. Abbildung 3.17b, Stickstoffgehalt: 220 ppm).

Erste Ergebnisse metallografischer Untersuchungen an Mikroschliffen im Randbereich gestanzter Löcher wurden von Valtinat; Dangelmaier (1993) vorgestellt. Die dort an heutigen Baustählen gewonnenen Erkenntnisse decken sich hinsichtlich der Beeinflussung des Grundgefüges mit denen an Flussstahl. Die Schliffbilder zeigen die typische Eindringfläche des Stanzstempels, deutlich zu erkennen an der Schnittausrundung an der oberen Lochkante. Der Scherbereich in Probendickenmitte ist wie der Austrittsbereich des Stempels an der Lochunterkante i. d. R. durch eine erhöhte Rauhigkeit des Lochrandes geprägt. Die größten Verformungen der Kornstruktur treten dabei in Probenmitte auf. Das Material verformt sich plastisch in Bewegungsrichtung des Stanzstempels. Dies ist deutlich an den in Verformungsrichtung gestreckten Ferritkörnern und an den daran ausgerichteten Mangansulfid-Teilchen 


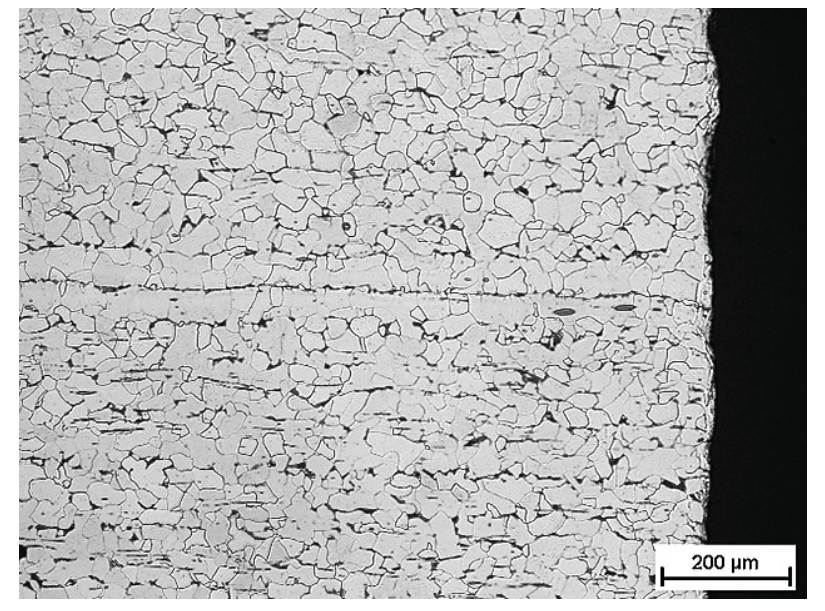

a) Probe PA2, Lochrand gebohrt, 100-fach

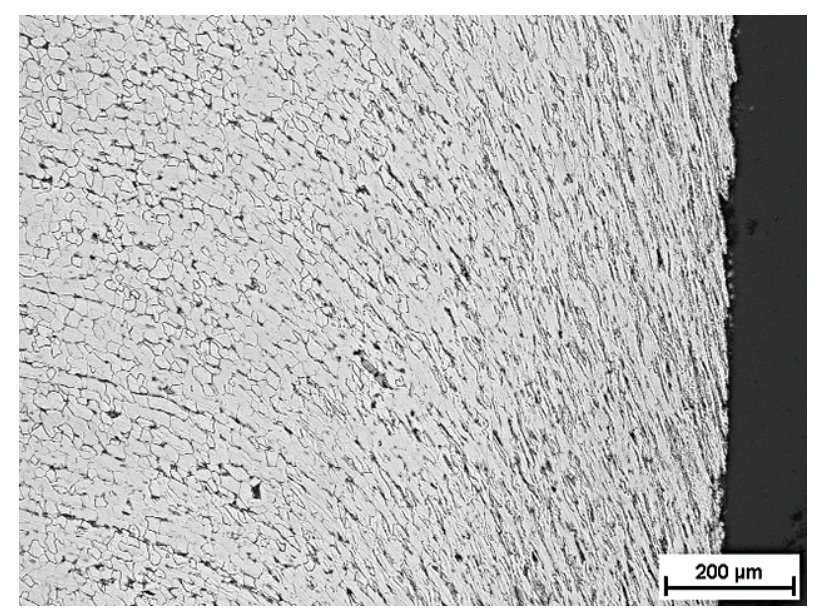

c) Probe SGM21, Lochrand gestanzt, 100-fach

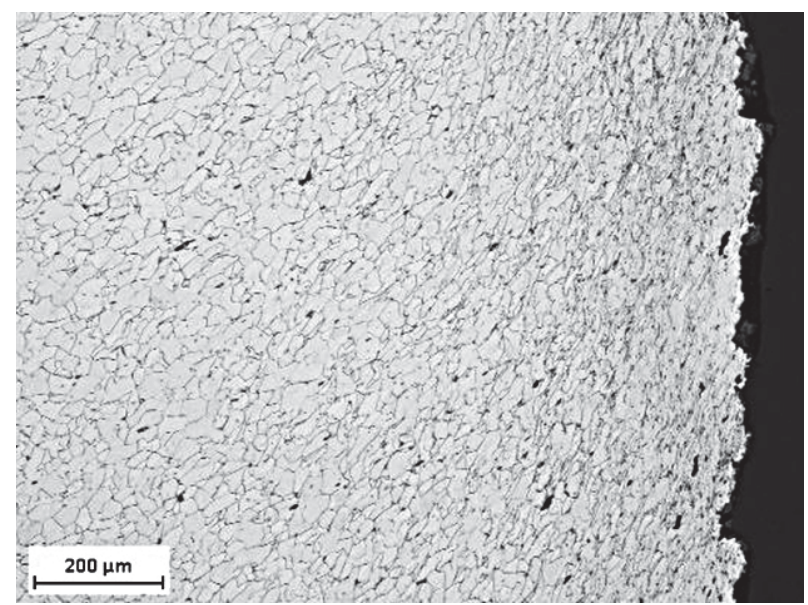

e) Probe E28, Bauteilrand geschert, 100-fach

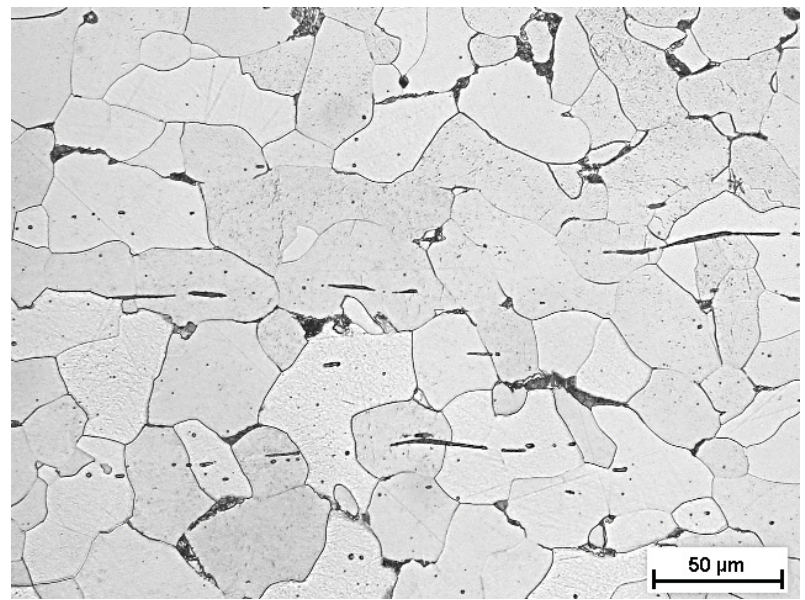

b) Probe PA2, unverformtes Grundgefüge mit gestreckten MnS-Einlagerungen, 400-fach

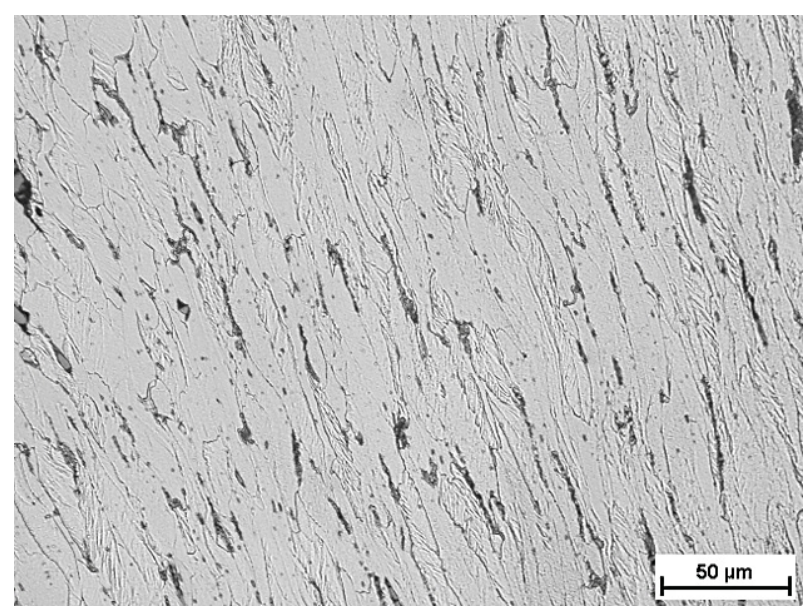

d) Probe SGM21, stark verformte und gestreckte Ferritkörner, 400-fach

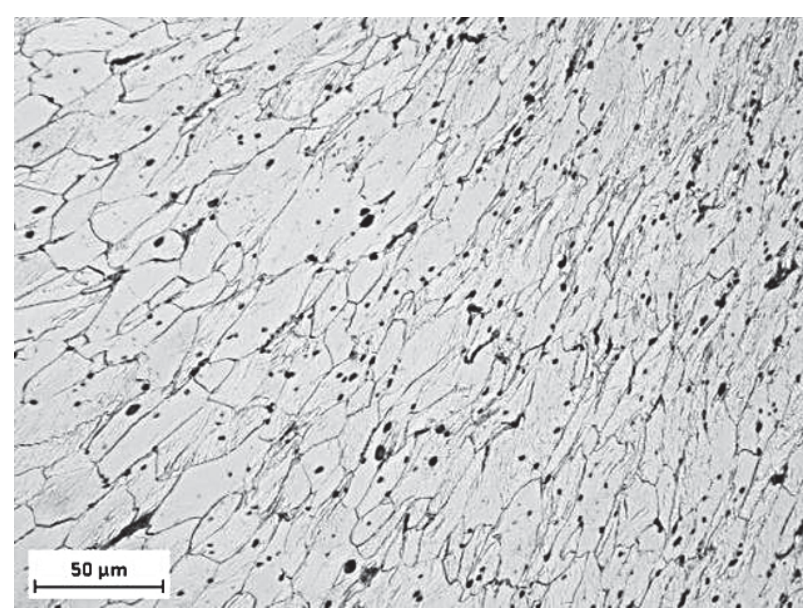

f) Probe E28, verformte und leicht gestreckte Ferritkörner, 400-fach

Abb. 3.17.: Gefügeaufnahmen an Loch- und Bauteilrändern nach Mikroätzung in alkoholischer $\mathrm{HNO}_{3}$-Lösung (Nitalätzung) 
erkennbar (siehe Abbildung 3.17c und d). Ähnliche Gefügezustände wurden an allen gestanzten Löchern wie auch schergeschnittenen Kanten (siehe Abbildung 3.17e und f) dokumentiert. Die Schliffbilder zeigten, dass die durch das Stanzen hervorgerufene Beeinflussung des Gefüges im Durchschnitt in einer Entfernung von etwa 1,5 mm vom Lochrand abgeklungen ist.

\subsubsection{Härteverlaufsmessungen}

Bereits in den Untersuchungen von Helms; Kühn et al. (1973), Valtinat; Dangelmaier (1993) und Huhn (2004) wurde die Verfestigung der Lochränder durch das Stanzen mit Hilfe von Härtemessungen nach Vickers quantifiziert. Gegenstand waren jedoch immer Baustähle neuerer Zeit ohne eine signifikante Neigung zur Reckalterung. Um die Übertragbarkeit der dabei gewonnenen Ergebnisse auf alte Stähle zu prüfen, wurden im Rahmen dieser Arbeit Härtemessungen an gestanzten oder schergeschnittenen Bauteilen aus Flussstahl durchgeführt.

Die Untersuchungen erfolgten an den fertig präparierten Mikroschliffen (siehe Abschnitt 3.5.2). Die Auswertung der Gefügeaufnahmen hatte ergeben, dass die verformte Zone jeweils in Mitte der Probendicke am ausgeprägtesten war. Daher wurden linienförmig Härteeindrücke in der Blechdickenmitte, ausgehend vom Loch- bzw. Bauteilrand, in das unverformte Grundgefüge hinein gesetzt. Um die Vergleichbarkeit mit den Ergebnissen der o. g. Veröffentlichungen zu ermöglichen, wurden Härtemessungen nach Vickers im Kleinlastbereich mit HV 0,2 (Prüfkraft: 2 N) gemäß DIN EN ISO 6507-1:2006 durchgeführt. Der Abstand der Messpunkte betrug jeweils $0,15 \mathrm{~mm}$.

Die grafisch aufbereiteten Ergebnisse aller Härteverlaufsmessungen sind in Abbildung 3.18 dargestellt. Für eine bessere Vergleichbarkeit der Proben untereinander wurden die absoluten Messwerte jeweils auf die Härte des unverfestigten Grundmaterials bezogen. Diese liegt, ähnlich wie in den Untersuchungen von HuHN (2004), zwischen 130 und 180 HV 0,2. In den verfestigten Randzonen steigt die Härte mit Maximalwerten von 325 HV 0,2 auf das bis zu 2,5 -fache an.

Diese Härtewerte deuten bereits auf eine beginnende Kaltrissneigung hin [SCHULZE (2010)], liegen aber noch unterhalb des nach DIN EN 1090-2:2011 zulässigen Höchstwertes von 380 HV 10. Darüber hinaus zeigen die Messergebnisse, dass mit steigender Werkstoffdicke eine erhöhte Randhärte zu verzeichnen ist. Im Unterschied dazu konnte keine Abhängigkeit der Ausdehnung der verfestigten Randzone von Werkstoff oder von der Bauteildicke nachgewiesen werden. Wie bereits bei den Untersuchungen von HELMS; KüHN et al. (1973) setzt der Einfluss des Stanzens bei einem Randabstand von etwa $3 \mathrm{~mm}$ ein. Die verfestigte Randzone ist somit deutlich breiter als der Bereich, in dem in den Mikroschliffen ein verformtes Grundgefüge nachgewiesen werden konnte.

Die Bestimmung von HV 0,2 gehört nach DIN EN ISO 6507-1:2006 zur sogenannten Kleinlasthärtemessung im Übergang zur Mikrohärte. Im Bereich der Kleinlasthärte wird unterhalb von HV 0,5 die ermittelte Härte zunehmend von der Prüfkraft abhängig und steigt gegenüber den Härtewerten im Makrobereich, z. B. HV 10 [Blumenauer (1994)]. Vernachlässigt man diesen, bei HV0,2 noch recht kleinen Prüfkrafteinfluss, erlaubt ISO 15653:2010 die Umrechnung der Vickershärte in die Zugfestigkeit $R_{m}$ nach Gleichung 3.32 .

$$
R_{m}=3,3 \cdot \operatorname{HV} 10-8 \text { für: } 100<\operatorname{HV} 10<400
$$




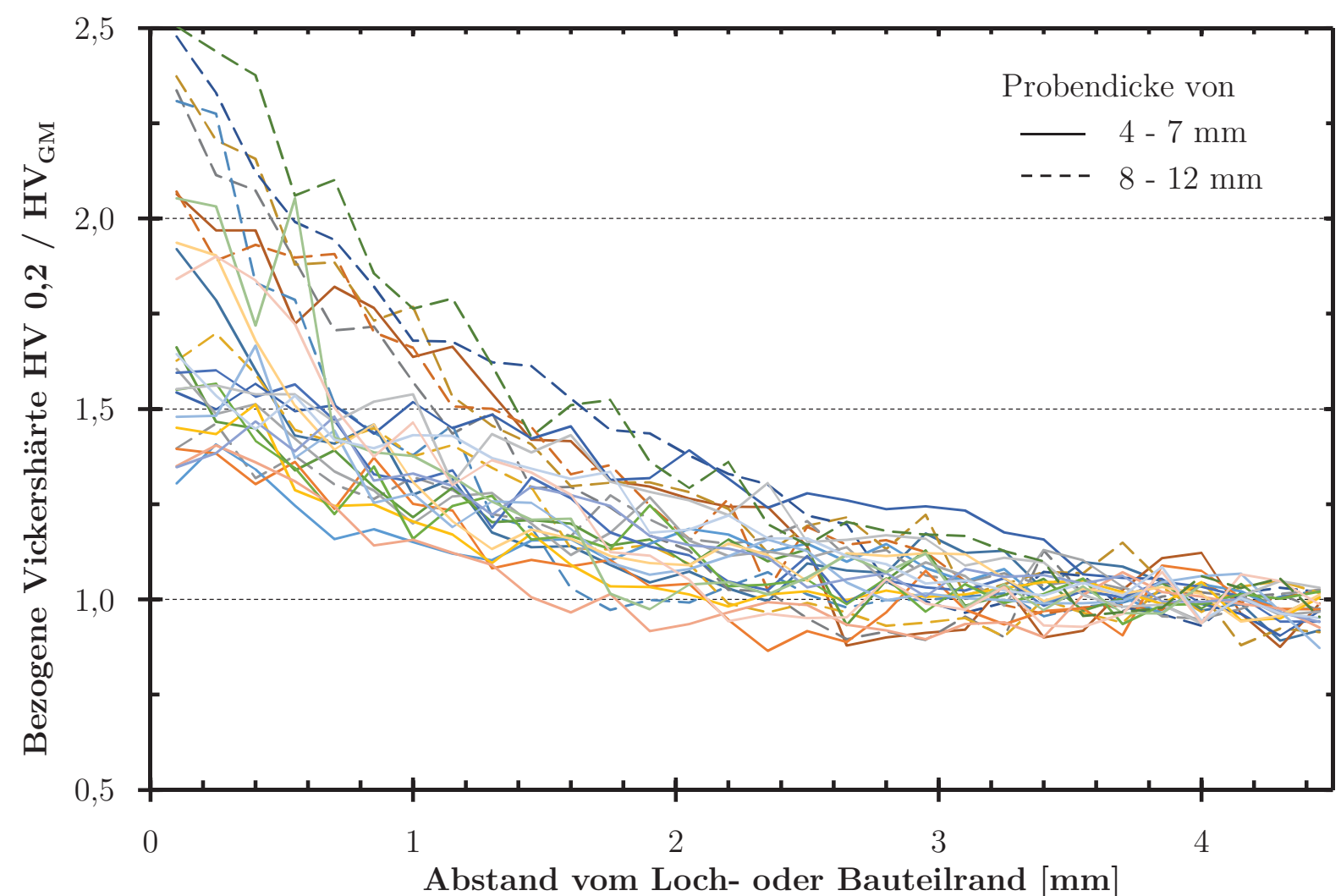

Abb. 3.18.: Härte-Tiefen-Verlauf aller untersuchten Proben, Härtewerte bezogen auf die Härte des unbeeinflussten Grundgefüges

Demnach führt die Kaltverfestigung durch das Stanzen der Löcher zu einer lokalen Festigkeitssteigerung auf bis zu $1000 \mathrm{~N} / \mathrm{mm}^{2}$. Dies bestätigen die Untersuchungsergebnisse von HeLms; KüHN et al. (1973) an den Rändern gestanzter Löcher.

\subsection{Bauteilzugversuche}

\subsubsection{Zugversuche an Winkelprofilanschlüssen mit einem Verbindungsmittel}

Ausgehend von den Erkenntnissen in Abschnitt 2.5.2 wurden zahlreiche Bauteilzugversuche bei tiefen Temperaturen durchgeführt, um ein Versagen durch Sprödbruch zu realisieren. Dabei fanden ausschließlich Bauteile aus Flussstahl aus Fachwerkkonstruktionen des Stahlhochbaus Verwendung. Primäres Kriterium bei der Auswahl des geeigneten Probenmaterials war dessen chemische Zusammensetzung, insbesondere der Gehalt an Stickstoff. Die chemische Analyse erfolgte, wie in Abschnitt 3.2 beschrieben, mittels optischer Emissionsspektrometrie. Die Ermittlung des Stickstoffgehalts erfolgte separat mit Hilfe der Trägergas-Heißextraktion. Ab einem Stickstoffgehalt von mehr als 120 ppm konnte von einer ausreichend starken Neigung des Stahls zur Alterung im Bereich gestanzter Nietlöcher ausgegangen werden [KÄPPLEIN; Wielgosch-FREy (2001)]. Das Materialgefüge einzelner Prüfkörper an den Lochrän- 
dern wurde nach den Zugversuchen im Rahmen der metallografischen Untersuchungen in Abschnitt 3.5 ebenfalls analysiert.

Bei der Mehrzahl der durchgeführten Bauteilzugversuche wurden Winkelprofile untersucht, die an einem Schenkel mit einem Niet angeschlossen waren. Ziel war es, das in den Abschnitten 4.3 und 5.3.1 abgeleitete, bruchmechanische Berechnungsmodell und den ihm zugrunde liegenden Versagensmechanismus zu überprüfen. Zur realitätsnahen Berechnung der Traglast der Bauteile erfolgte vor der Versuchsdurchführung eine Vermessung der Querschnitts- und Anschlussgeometrie aller Versuchskörper. Außerdem wurden die mechanischen Kennwerte des Probenmaterials in Zugversuchen nach DIN EN ISO 6892-1:2009 bestimmt.

Um den Schwierigkeiten bei der Beschaffung geeigneter Versuchskörper zu begegnen, wurden, so weit möglich, aus jedem geeigneten Bauteil je 2 Prüfkörper aus den beiden Endstücken der Winkelprofile hergestellt. Um das Versagen im vorhandenen Nietloch sicherzustellen, wurde am zweiten Prüfkörperende ein Anschluss mit mindestens 2 Verbindungsmitteln kleineren Durchmessers hergestellt. Durch die Verwendung hochfester Schrauben konnte ein Versagen der Verbindungsmittel ausgeschlossen werden. Die Lasteinleitung an beiden Anschlüssen der Prüfkörper erfolgte über Flachstähle 100 x 10 mm aus S355.

Nach dem Verschrauben der Prüfkörper mit den Anschlussadaptern wurden sie in einer Klimakammer auf ca. $10^{\circ} \mathrm{C}$ unterhalb der geplanten Versuchstemperatur heruntergekühlt. Die Kühlung erfolgte gemeinsam mit den Anschlussblechen, um eine zu schnelle Erwärmung während des Einbaus in die Prüfmaschine zu verhindern. Anschließend wurden die Prüfkörper in einer servo-hydraulischen Prüfmaschine (max. Prüfkraft: $250 \mathrm{kN}$ ) eingebaut, nach Erreichen der geplanten Prüftemperatur im Anschlussbereich der Zugversuch gestartet und die Traglast des Bauteils ermittelt.

Nach dem Ausbau der Prüfkörper wurde die Art des Versagens im Anschluss protokolliert. Dabei wurde zwischen folgenden Bruchtypen unterschieden:

- Bruchtyp L-SS: Sehr spröder Bruch, ausgehend vom seitlichen Lochrand mit einem durchgängigen, verformungsfreien Bruchverlauf quer durch das Profil bis in den ungelochten Winkelschenkel,

- Bruchtyp L-S: Spröder Bruch, ausgehend vom seitlichen Lochrand als Trennung mit konstanter Spaltöffnung ohne Einschnürung,

- Bruchtyp L-HS: Halbspröder Bruch am seitlichen Lochrand, als spröder Anriss, ausgehend vom Lochrand, der sich mit ansteigender Zugkraft und zunehmender Lochovalisierung nach innen öffnet, während die Schenkelkontur einschnürt,

- Bruchtyp L-D: Duktiler Bruch des seitlichen Lochrandes, der eine Einschnürung mit Rissbeginn erst nach großer Lochovalisierung zeigt, kein spröder Initialriss am Lochrand

Diese vier Bruchtypen sind exemplarisch in Abbildung 3.19 dargestellt. Neben dem Versagen am lateralen Lochrand wurde bei einzelnen Prüfkörpern auch ein Aufreißen der axialen Lochleibung, von der Stirnseite des Winkelprofils zum Nietloch hin, beobachtet (ähnlich Abbildung 3.19 b). Dieser Versagensmechanismus wird ebenso wie das vollduktile Versagen im Rahmen dieser Untersuchungen nicht weiter berücksichtigt.

Im Anhang B sind die Ergebnisse der Traglastversuche ebenso wie die aufgemessenen Bauteilgeometrien sowie die Streckgrenze und Zugfestigkeit des Grundmaterials tabellarisch zusammengefasst. 


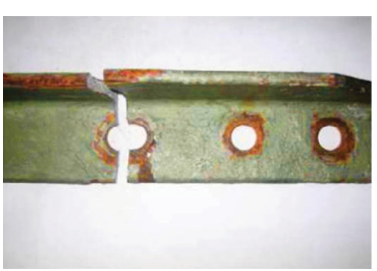

a) Bruchtyp L-SS

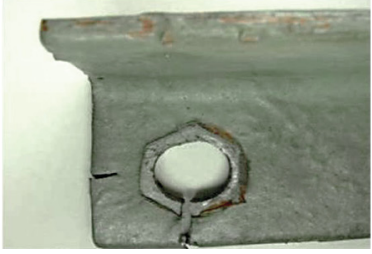

b) Bruchtyp L-S

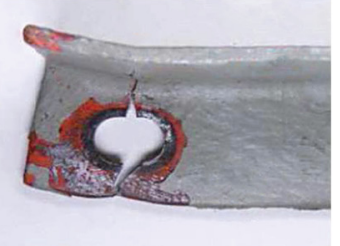

c) Bruchtyp L-HS

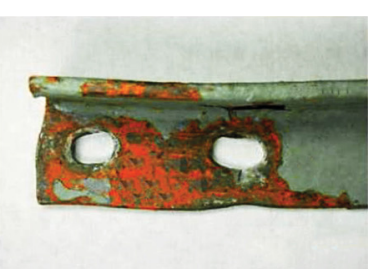

d) Bruchtyp L-D

Abb. 3.19.: Bruchtypen bei Versagen im Bauteilzugversuch vom seitlichen Lochrand der Winkelprofile

\subsubsection{Zugversuche an Winkelprofilanschlüssen mit mehreren Verbindungsmitteln}

Um das in Abschnitt 5.3.2 aufgestellte Berechnungsmodell für zentrisch zugbeanspruchte Winkelprofile mit Anschlüssen aus mehreren Verbindungsmitteln zu validieren, wurden derartige Bauteile ebenfalls in Zugversuchen geprüft. Die Vorbereitung und Durchführung der Versuche erfolgte analog zu den Versuchen mit einem Verbindungsmittel. Die Anschlüsse der Bauteile bestanden aus 8 bis 10 Verbindungsmitteln, die zu gleichen Teilen auf die beiden Winkelschenkel verteilt waren. Aufgrund von teilweise begrenzten Bauteillängen konnte an einzelnen Prüfkörpern nur an einem Bauteilende ein geschraubter Anschluss mit Anschlusslaschen montiert und geprüft werden. Der Anschluss am zweiten Probenende erfolgte dann durch Verschweißen mit den Anschlussblechen. Bei der Dimensionierung der Anschlussschweißnähte wurde darauf geachtet, dass ein Versagen des Bauteils im geschraubten Anschluss stattfindet. Die technologische Ausführung der Schweißung der alten Flussstähle erfolgte unter Berücksichtigung der Empfehlungen von LüDDECKE (2006) und WTA E-7-305/D:2005.

Die Ergebnisse dieser Bauteilzugversuche sind im Anhang B tabellarisch zusammengefasst. Abbildung 3.20 zeigt ein repräsentatives Bruchbild eines untersuchten Winkelprofils. Ausgehend vom höchst beanspruchten Nietloch verlief der Bruch rechtwinklig zur Bauteilachse durch den Winkelquerschnitt hindurch bis zum gegenüberliegenden Bauteilrand.

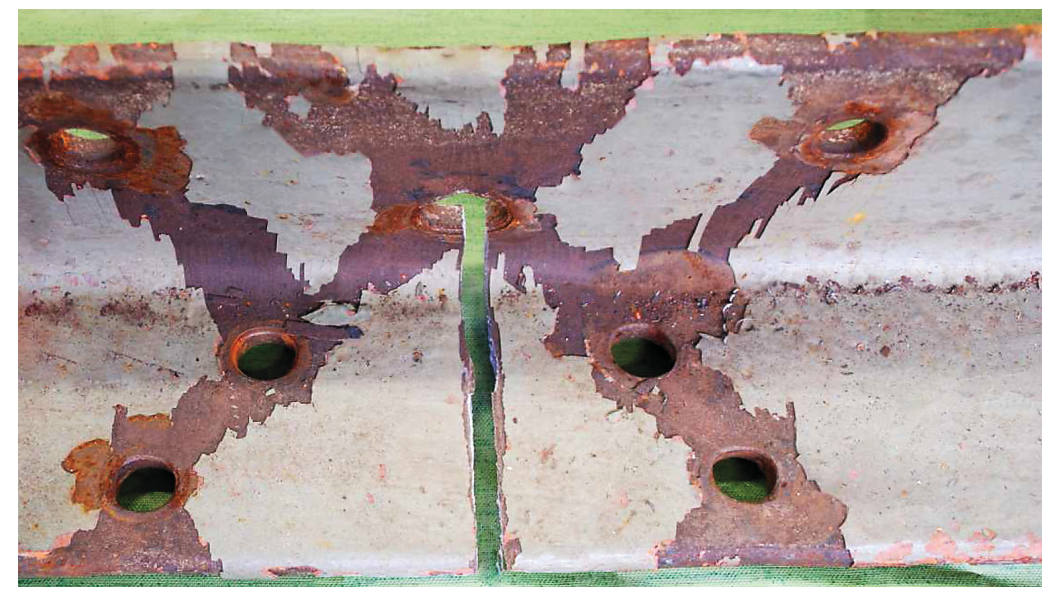

Abb. 3.20.: Bruchbild eines zentrisch gezogenen Winkelprofils im Bauteilzugversuch bei Anschluss mit mehreren Verbindungsmitteln 



\section{Kapitel 4.}

\section{Numerische Untersuchungen von Winkelprofilanschlüssen}

\subsection{Grundlagen bruchmechanischer Finite-Element-Berechnungen}

Wesentlicher Bestandteil der FE-Modellierung für bruchmechanische Berechnungen ist eine geeignete Modellierung der Rissspitze und Rissfront sowie eine mit dieser Modellierung verträgliche Auswertung der Ergebnisse zur Bestimmung der Rissspitzenbeanspruchung. Zur Vernetzung des Berechnungsmodells an der Rissspitze, für Untersuchungen nach der linearelastischen Bruchmechanik, empfehlen Anderson (1995) und KunA (2010) die Verwendung von degenerierten „elastic singularity elements“. Dabei handelt es sich im Zweidimensionalen um Rechteckelemente mit 8-Knoten, bei denen die Knoten einer Kante (1, 4 und der Mittelknoten 8) auf einen Punkt verschoben werden. Dadurch entsteht ein degeneriertes (oder auch kollabiertes) Element mit dreieckiger Form, dessen neu entstandene Spitze an der Rissspitze angeordnet wird. Die Verschiebungen dieser drei Knoten sind fest miteinander gekoppelt. Darüber hinaus sind die Mittelknoten 5 und 7 der benachbarten Seiten in die Viertelspunkte der Elementkanten zur Rissspitze hin verschoben (siehe Abbildung 4.1b). Diese Konfiguration verursacht eine $1 / \sqrt{r}$ Singularität der Verzerrungen auf den beiden modifizierten Kanten, die zur Modellierung der Spannungskonzentration an der Rissspitze und damit zur Berechnung des Spannungsintensitätsfaktors K erforderlich ist. Da diese Singularität nur auf den Elementkanten, also in radialer Richtung, besteht, müssen die kollabierten Elemente in ausreichender Anzahl fächerförmig um die Rissspitze herum angeordnet werden.

Rissspitzenelemente für Berechnungen nach der elastisch-plastischen Bruchmechanik unterscheiden sich darin, dass die Knoten 1, 4 und 8 nicht miteinander verbunden sind und sich somit unabhängig voneinander verformen können. Diese Art der Modellierung ermöglicht die Öffnung und die als „blunting“ bezeichnete Abstumpfung des Risses (vgl. BeLTRAmi (1995)). Die Mittelknoten 5 und 7 auf den radialen Elementkanten befinden sich unverändert in der Mitte der Elementlänge (siehe Abbildung 4.1c). Durch diese Knotenkonfiguration des Elements wird an den auf die Rissspitze zulaufenden Elementkanten eine $1 / r$ Singularität der Verzerrungen modelliert, die es ermöglicht, auch ideal plastisches Verhalten abzubilden.

Für die Modellierung von gekrümmten Rissfronten, wie auch allgemein für die Untersuchung räumlicher Bauteile, wie es in dieser Arbeit vorgesehen ist (siehe Abschnitt 4.2), ist die Anwendung von Volumenelementen erforderlich. In Abbildung 4.2 ist eine räumliche Rissspitzenmodellierung veranschaulicht. Analog zu den oben beschriebenen 2D-Elementen wird 
das verwendete Hexaeder-Element derart kollabiert, dass zwei benachbarte Elementkanten aufeinander fallen und diese im Modell entlang der Rissfront angeordnet werden. Je nach zu untersuchendem Werkstoffverhalten (linear-elastisch oder elastisch-plastisch) erfolgt die Anordnung der Zwischenknoten auf den radial verlaufenden Elementkanten wie zuvor beschrieben.

a)

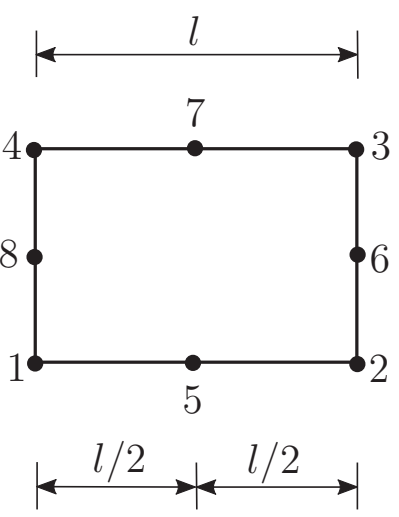

b)
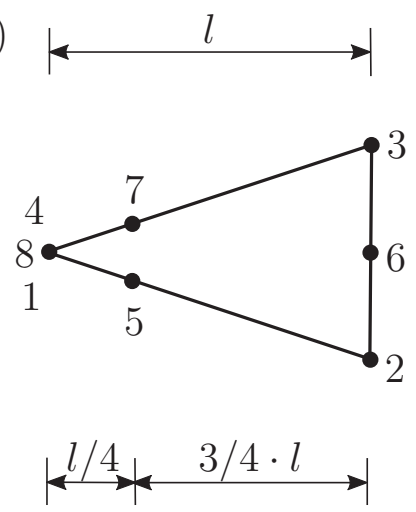

c)
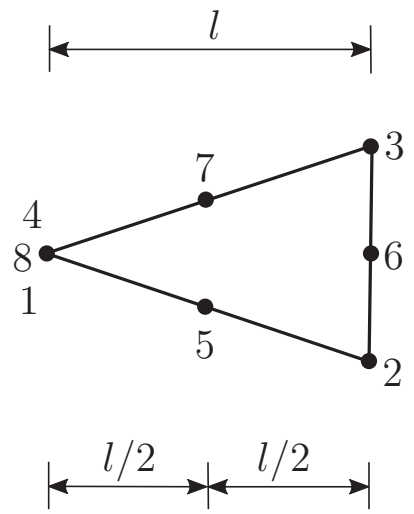

Abb. 4.1.: Isoparametrische Rissspitzenelemente

a) gewöhnliches zweidimensionales 8-Knoten-Element

b) kollabiertes 8-Knoten-Element mit 1/4-Punkt-Knoten für linear-elastische Berechnungen $(1 / \sqrt{r}$-Singularität)

c) kollabiertes 8-Knoten-Element mit 1/2-Punkt-Knoten für elastisch-plastische Berechnungen $(1 / r$-Singularität)

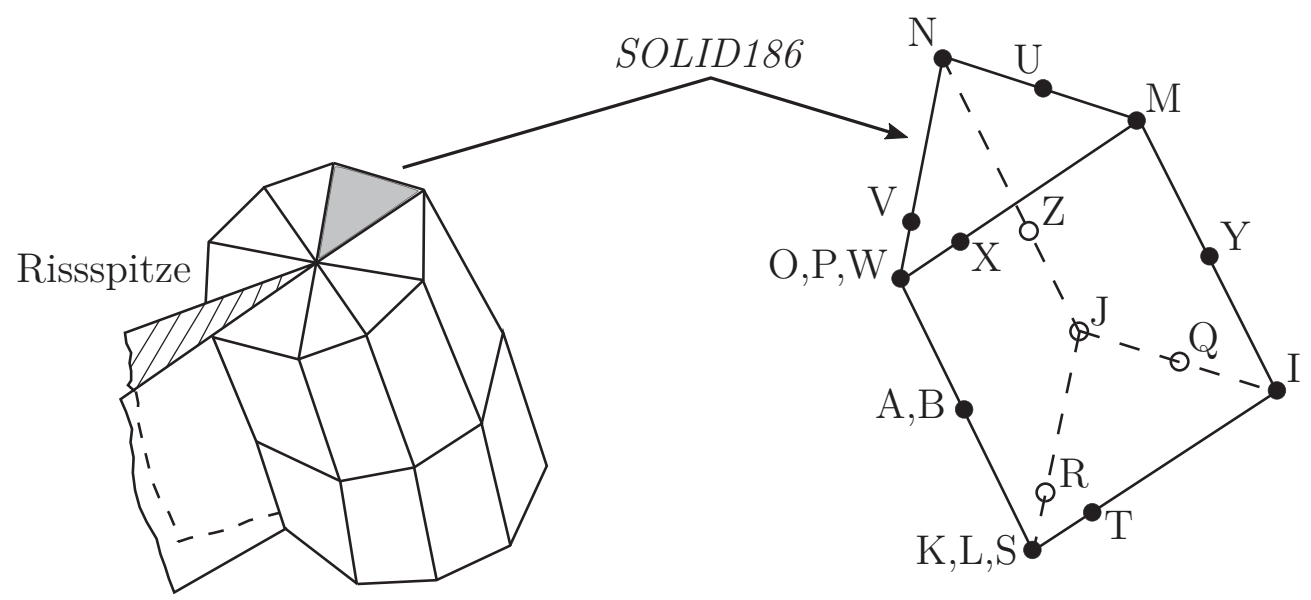

Abb. 4.2.: Isoparametrisches Rissspitzenelement als kollabiertes Hexaeder-Element zur Modellierung räumlicher Risse und Strukturen

\subsection{Modellierung der Winkelprofilanschlüsse}

Die im Folgenden beschriebene Modellgenerierung und die numerischen Berechnungen wurden mit dem Finite-Elemente-Analyse-Programm ANSYS 14.5 durchgeführt. Die anschließende Auswertung der Berechnungsergebnisse erfolgte entweder im Postprocessing von ANSYS oder durch Auslesen der Ergebnisse und anschließende Weiterverarbeitung in Microsoft EXCEL. 
Zur Steuerung der numerischen Berechnungen wurde die ANSYS-Parametersprache (ANSYS Parametric Design Language, kurz APDL) genutzt. Die Anwendung von APDL ermöglichte es, die Geometrie der Berechnungsmodelle parametrisch zu generieren. Die erforderlichen Parameter-Kombinationen konnten somit durch Einbettung in Schleifen (siehe Groth; MüLleR (2009)) in einem Durchlauf berechnet werden.

ANSYS bietet mit dem Befehl KSCON für bruchmechanische Untersuchungen die Möglichkeit, in einem ebenen Modell kollabierte Elemente (siehe Abschnitt 4.1) um einen Keypoint fächerförmig zu erzeugen, an die wiederum ein zweiter Ring von Viereckelementen anknüpft. Dabei sind der Radius der ersten Reihe, die Elementgröße der zweiten Reihe, die Anzahl der ringförmig angeordneten Elemente und die Position der Mittelknoten auf den radialen Elementkanten des inneren Rings einstellbar. Diese Art der Modellierung bildet die Grundlage für die von ANSYS ebenso zur Verfügung gestellte Berechnung des Spannungsintensitätsfaktors mit dem Befehl $K C A L C$. Dieser ermittelt den K-Faktor aus den Verschiebungen der Knoten der ersten beiden Elemente auf den Rissufern [ANSYS (2014)]. Dieses Verfahren liefert für ebene Modelle ausreichend genaue Ergebnisse.

Alternativ bietet ANSYS die Möglichkeit, den Spannungsintensitätsfaktor $K_{I}$ über die Berechnung des J-Integrals mit der programminternen Routine CINT zu ermitteln. Dieses Verfahren basiert auf der von SHiH; Moran et al. (1986) entwickelten Domain-IntegralMethode, in der im ebenen Modell das Linienintegral nach Gleichung 2.11 durch ein Flächenintegral und in räumlichen Modellen das entsprechende Flächenintegral durch ein Volumenintegral ersetzt wird. Das Verfahren berechnet, ausgehend von einer fiktiven Rissverlängerung, über das entsprechende Gebietsintegral die Energiefreisetzungsrate. Bei der Modellierung muss deshalb neben der Rissfront auch die Rissfortschrittsrichtung definiert werden. Außerdem ist noch die Anzahl der Konturen anzugeben, für die das Gebietsintegral ermittelt werden soll. Die Konturen beschreiben „Ringe“ von Elementen um die Rissspitze herum (siehe Abbildung 4.4), von einem Rissufer zum anderen, wobei die erste Kontur die unmittelbar an der Rissspitze befindlichen Elemente enthält.

Für die in dieser Arbeit untersuchten Winkelprofile ist eine dreidimensionale Modellierung notwendig, um die Beanspruchung infolge des exzentrischen Anschlusses und die versteifende Wirkung des freien Winkelschenkels realitätsnah abzubilden. Die in Abschnitt 4.1 beschriebene Viertelpunktmethode zur Modellierung der Rissspitze ist nach KunA (2010) für dünnwandige Platten und Schalen, unter Verwendung regulärer Schalenelemente, nicht anwendbar. Aus diesem Grund werden für die erforderliche 3D-Modellierung der Winkelprofile Hexaeder-Elemente in einem Volumenmodell verwendet.

Die zuvor beschriebene Vernetzung der Rissspitze, mit Elementen mit verschobenem Mittelpunktknoten der radialen Elementkanten, macht bei einer Modellierung mit Volumenelementen den Einsatz von 20-Knoten Hexaeder-Elementen SOLID186 für das gesamte Modell erforderlich. Um die Anzahl der Freiheitsgrade des Gesamtmodells und die damit verbundene Rechenzeit für die geplante Parameterstudie zu verringern, wurde nach den Empfehlungen von KunA (2010) stattdessen eine Modellierung mit 8-Knoten Hexaeder-Elementen SOLID185 mit linearen Ansatzfunktionen durchgeführt. Hierbei war jedoch eine deutlich feinere Vernetzung des Nahfeldes um die Rissspitze notwendig.

Um die Feinheit der Vernetzung des Modells diesbezüglich gezielt beeinflussen zu können, wurde es in mehrere Teilsegmente unterteilt (siehe Abbildung 4.3). Wesentlicher Bestandteil ist dabei das zylindrische Segment an der Rissspitze („Rissschlauch“), welches bei allen Modellen der Parameterstudie identisch modelliert wurde. Um die Rissspitze herum waren 
insgesamt 16 kollabierte Elemente, mit daran anknüpfenden Hexaeder-Elementen, fächerförmig in 12 Kreisringen angeordnet. Die Länge der radialen Elementkanten betrug jeweils $0,1 \mathrm{~mm}$. Bei der zu modellierenden Risslänge von $a=3 \mathrm{~mm}$ (siehe Abschnitt 4.3) entspricht dies einer Länge von $a / 30$.
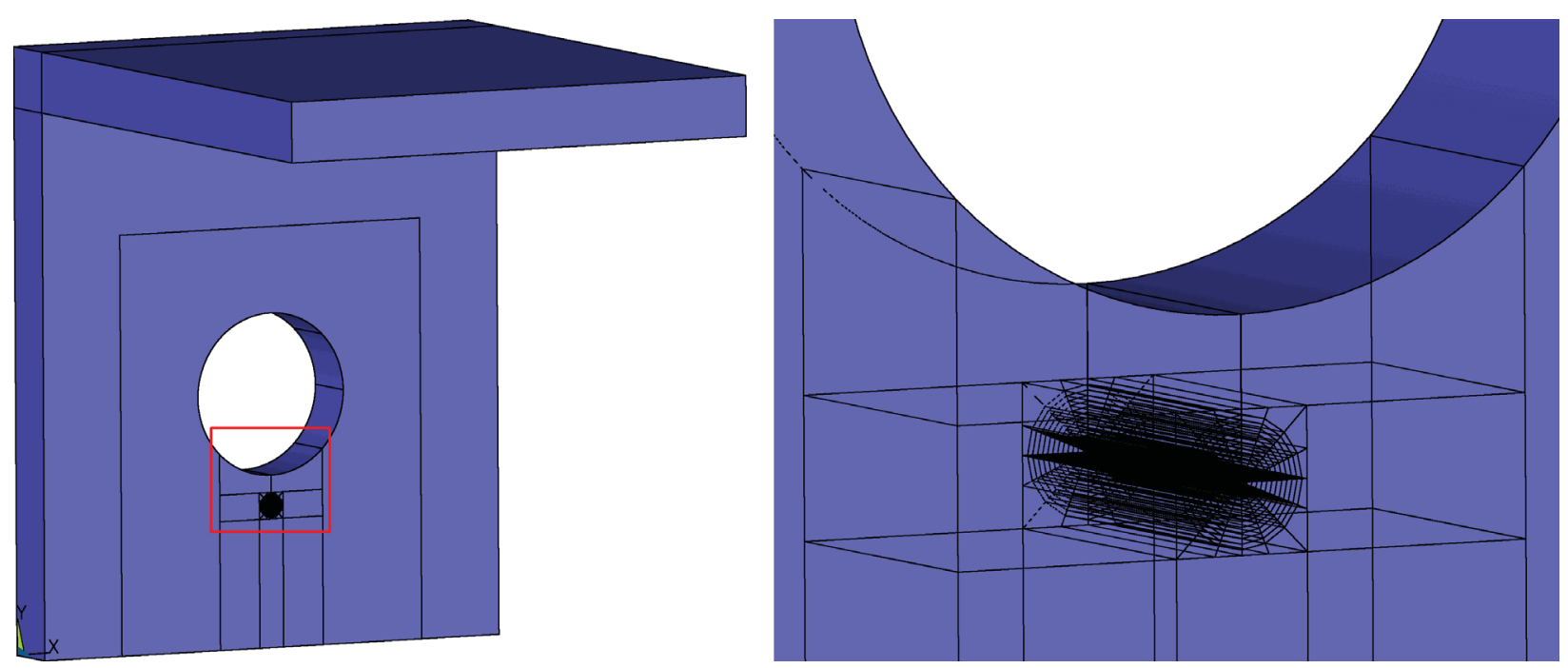

Abb. 4.3.: Teilkörper des Berechnungsmodells im Bereich des angerissenen Lochs

Mit diesem sehr feinen Netz an der Rissspitze (siehe Abbildung 4.4) konnten trotz des Einsatzes der einfacheren 8-Knoten-Elemente ausreichend genaue Ergebnisse des Spannungsintensitätsfaktors berechnet werden. Von Freitag (2013) wurde an Testmodellen in Form von $\mathrm{C}(\mathrm{T})$ - und $\mathrm{M}(\mathrm{T})$-Proben nachgewiesen, dass bei einer derartigen Vernetzung an der Rissspitze sehr gute Übereinstimmungen mit vorhandenen K-Faktor-Lösungen aus der Fachliteratur (z. B. Al Laham; Ainsworth et al. (1998) und Berger; Blauel et al. (2006)) erzielt wird.

Für die Segmente des Modells um das Nahfeld herum wurde die Netzfeinheit durch Vorgabe von Teilungen auf den Begrenzungslinien festgelegt. Damit war es möglich, unter Nutzung des von ANSYS zur Verfügung gestellten, automatischen Netzgenerators, das Modell mit wachsendem Abstand von der Rissspitze zunehmend gröber zu vernetzen. Dies gilt auch für den horizontalen, ungelochten Schenkel. In Abbildung 4.5 ist für ein Winkelprofil die Diskretisierung des Gesamtmodells dargestellt.

Die sehr feine Vernetzung, insbesondere im Bereich des Anrisses, führt zu hohen Elementund Knotenanzahlen. Um die Anzahl der Freiheitsgrade der FE-Modelle und die damit verbundene Rechenzeit weiter zu minimieren, wurde der Einfluss der Modell- bzw. Profillänge in Testrechnungen von FREITAG (2013) überprüft. Die Untersuchungen zeigten, dass die Länge des Modells keinen Einfluss auf die Ergebnisse der Rissspitzenbeanspruchung hat. Deshalb wurde die Länge der Winkelprofile in den Berechnungsmodellen auf die fünffache Schenkelbreite festgelegt und damit gegenüber realen Bauteilen deutlich verkürzt.

Neben der geeigneten Rissmodellierung ist die Rissspitzenbeanspruchung auch von der realitätsnahen Modellierung der Lastübertragung durch den Nietbolzen im Loch abhängig. Um die aufwendige Berechnung des Kontaktproblems zwischen Bolzen und Lochleibung in den zahlreichen FE-Berechnungen der Parameterstudie zu umgehen, bestand die Notwendigkeit nach einer alternativen Modellierung der Lasteinleitung. Basierend auf den Untersuchungen 


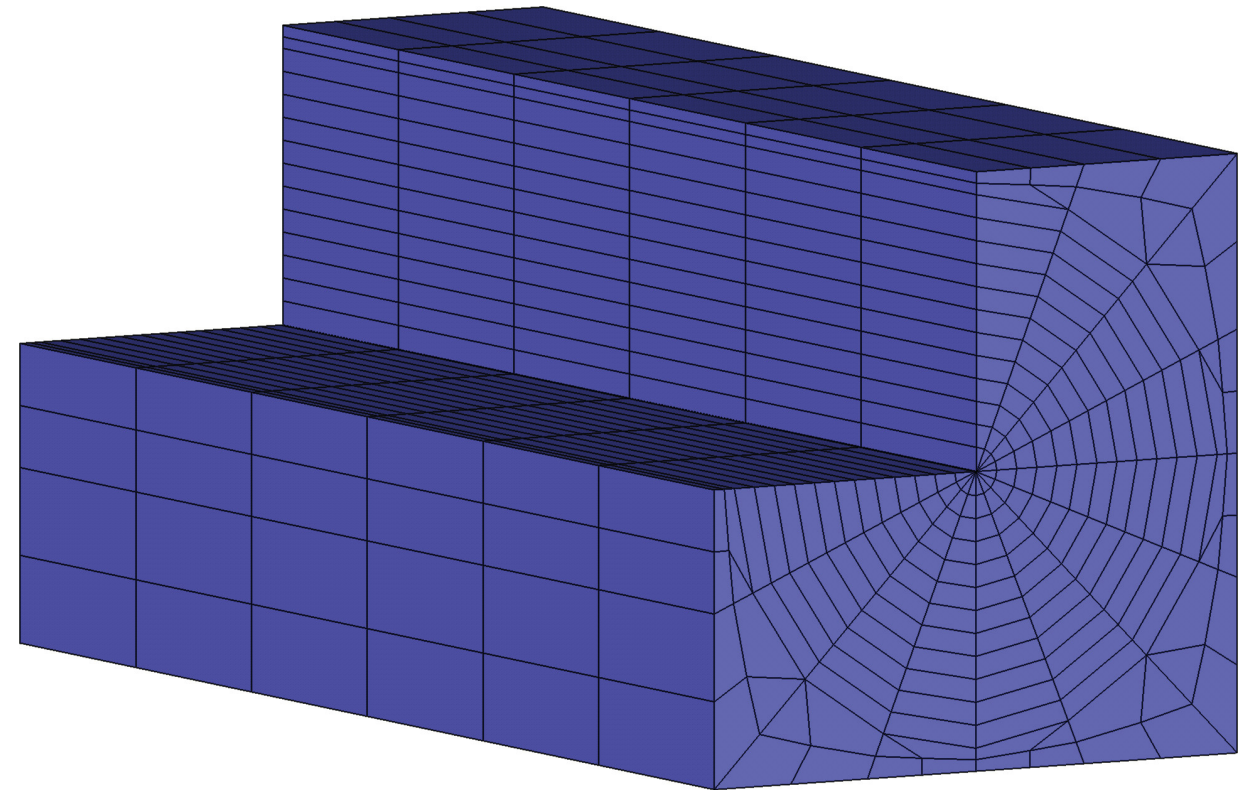

Abb. 4.4.: Modellausschnitt mit Vernetzung der Rissspitze

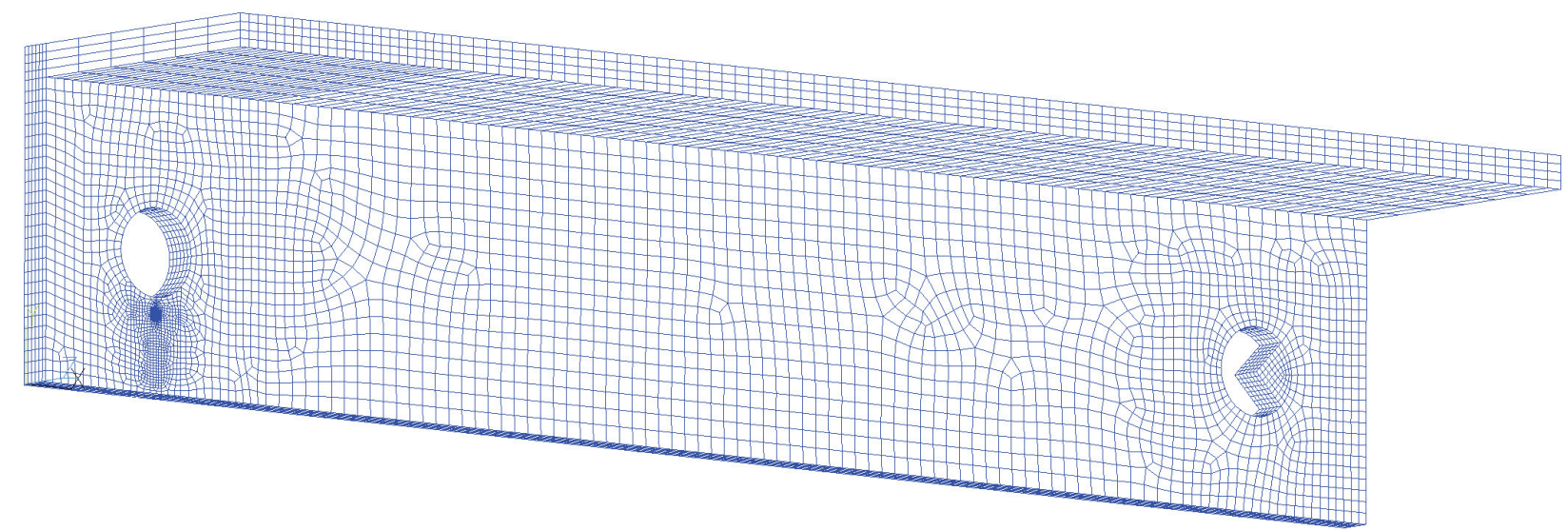

Abb. 4.5.: Vernetzung des Gesamtmodells

von Tweed; Rooke (1979), Rooke; Tweed (1980) und WANLin (1993) wurde die Lochleibungslast als radiale, linear veränderliche Last über die halbe Lochwandung angesetzt (siehe Abbildung 4.6). Vergleichsberechnungen von HUHN (2004) ergaben sehr gute Übereinstimmungen der Nettospannungen in der Rissachse gegenüber einer Modellierung mit Kontakt zwischen Bolzen und Lochleibung.

Die Modellierung des Bolzens im zweiten, nicht angerissenen Nietloch des Modells orientiert sich an den Empfehlungen von KunA (2010). Entsprechend wurde im Bereich der belasteten Lochleibung ein Viertel Kreissegment angeordnet (siehe Abbildung 4.7). Im Kontaktbereich erfolgte der Anschluss des Viertelkreises an das vorhandene Netz. Dem Kreissegment wurden linear elastische Materialeigenschaften mit erhöhtem E-Modul zugewiesen, um unrealistisch hohe Verformungen in der Lasteinleitungszone zu vermeiden. Die Lagerung des Modells in Bauteillängsrichtung erfolgte an den Knoten des Kreismittelpunktes. Damit konnte ein Verdrehen des Profils um die Achse des Verbindungsmittels ermöglicht werden. 


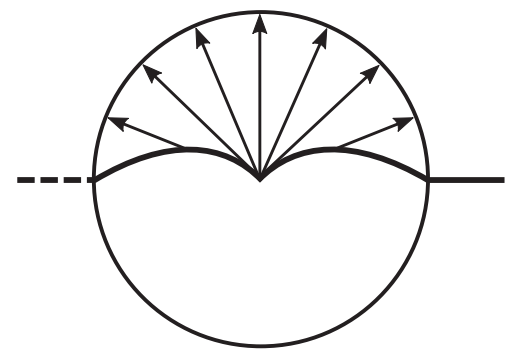

Abb. 4.6.: Modellierung der Lochleibungsspannung im Loch mit Anriss [Rooke; Tweed (1980)]

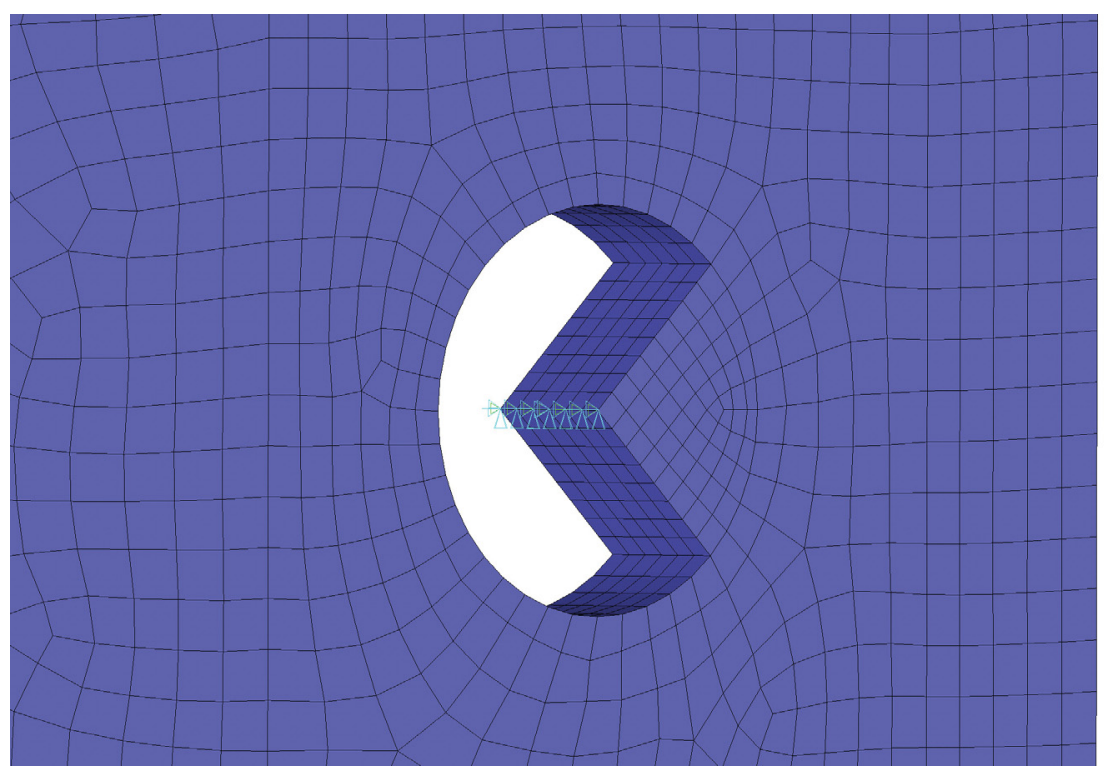

Abb. 4.7.: Modellierung der Lasteinleitung und Lagerung am ungerissenen Loch

Die gewalzten Ausrundungen der Winkelprofile an den Rändern und am Übergang zwischen den Schenkeln haben auf die Beanspruchung an der Rissspitze einen vernachlässigbaren Einfluss und wurden deshalb vereinfachend im Modell nicht berücksichtigt.

Die Berechnung der Rissspitzenparameter $K_{I}$ und $J_{e}$ erfolgte unter Ansatz linear elastischen Werkstoffverhaltens mit dem Elastizitätsmodul $E=210000 \mathrm{~N} / \mathrm{mm}^{2}$ und der Querkontraktionszahl (Poissonzahl) $\mu=0,3$.

\subsection{FEM-Parameterstudie}

Wie in Abschnitt 2.3 beschrieben, bestehen viele Bauteile in schlanken Fachwerkkonstruktionen des Stahlhochbaus aus Winkelprofilen, die durch Niete an einem Schenkel angeschlossen sind. Die durch diesen exzentrischen Anschluss hervorgerufene Biegebeanspruchung, verstärkt durch die im Schenkel meist außermittige Anordnung der Nietlöcher und den versteifend wirkenden, abstehenden Winkelschenkel, hat zur Folge, dass derartige Bauteile nicht mit den üblicherweise für Nietkonstruktionen verwendeten, bruchmechanischen Berechnungsmodellen beschrieben werden können. Von Hensen (1992) wurden verschiedene Konstruktions- 
details genieteter Eisenbahnbrücken mit Anriss am Loch- oder Bauteilrand untersucht und auf die drei in Abbildung 2.16 dargestellten Modelle zurückgeführt. Die darin als konstant angenommene Zugbeanspruchung, bei gleichzeitiger Durchleitung der gesamten Zugkraft ohne Lasteinleitung am Loch, ist bei den hier zu analysierenden Anschlüssen von Winkelprofilen nicht gegeben.

Ausgehend von der maximalen Kerbspannung werden die Anschlüsse der Winkelprofile mit einem Anriss am Rand der Nietlöcher untersucht. Dabei wird, wie für dünnwandige, genietete Bauteile üblich, von einem Durchriss mit gerader Rissfront ausgegangen. Aufbauend auf den Ergebnissen in Abschnitt 3.5 zur Verfestigung gestanzter Lochränder wird im Folgenden die Beanspruchung der Winkelprofile bei einer konstanten Länge des Anrisses von $a=3 \mathrm{~mm}$ untersucht. Dieser Riss wird in der höher beanspruchten Lochwandung im schmaleren Bereich des gelochten Schenkels modelliert (siehe Abbildung 4.8). Auf Basis der in Abschnitt 2.3 analysierten, typischen Anschlussdetails in bestehenden Stahlfachwerk-Konstruktionen, wurden in einer Parameterstudie für 7 verschiedene Winkelprofilquerschnitte insgesamt 170 Geometrie-Konfigurationen numerisch untersucht. Die dabei betrachteten Parameterbereiche der Profil- und Anschlussabmessungen sind in Tabelle 4.1 zusammengefasst.
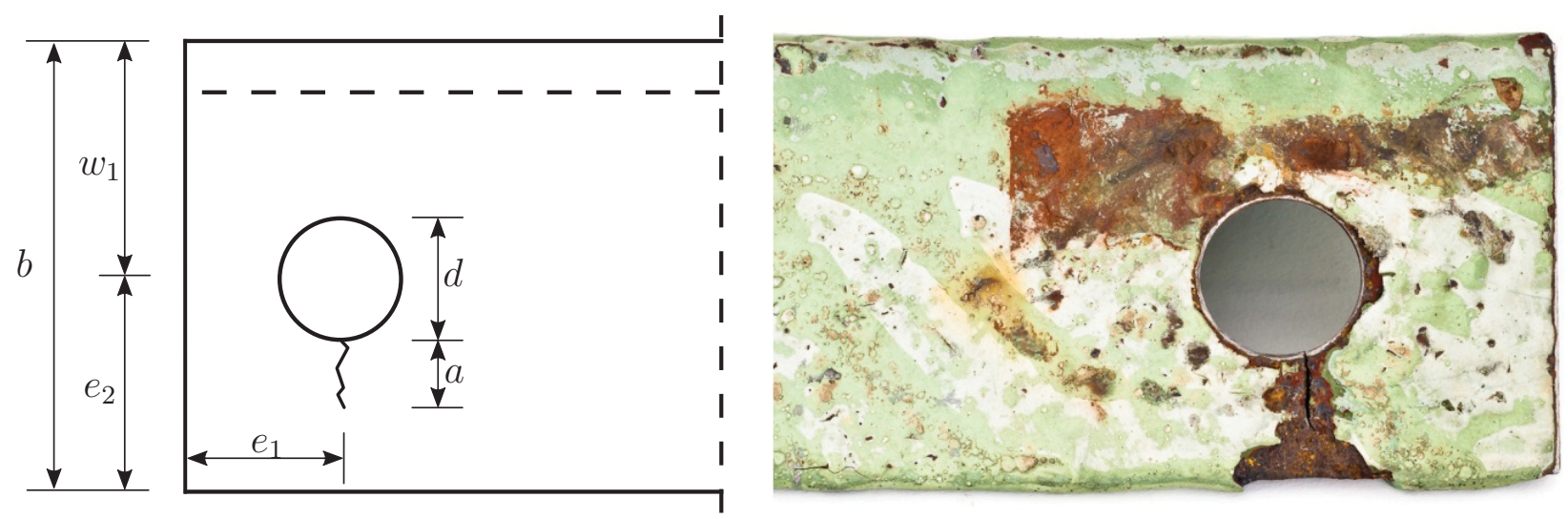

Abb. 4.8.: Geometrie des Winkelanschlusses mit einem Verbindungsmittel und Anriss am Lochrand

Tab. 4.1.: Geometrieparameter der numerischen Untersuchungen an Winkelprofilanschlüssen mit einem Verbindungsmittel

\begin{tabular}{|c|c|c|c|}
\hline Winkelquerschnitt & $d[\mathrm{~mm}]$ & $e_{1}[\mathrm{~mm}]$ & $e_{2}[\mathrm{~mm}$ \\
\hline $\begin{array}{l}\mathrm{L} 40 \times 4 \\
\mathrm{~L} 50 \times 5 \\
\mathrm{~L} 60 \times 6 \\
\mathrm{~L} 65 \times 7 \\
\mathrm{~L} 70 \times 7 \\
\mathrm{~L} 80 \times 8 \\
\mathrm{~L} 90 \times 9\end{array}$ & $\begin{array}{l}12-14 \\
12-17 \\
16-21 \\
16-24 \\
16-24 \\
20-26 \\
26-29\end{array}$ & $\begin{array}{l}20-25 \\
20-25 \\
25-30 \\
30-35 \\
30-35 \\
35-40 \\
40-50\end{array}$ & $\begin{array}{l}17-20 \\
20-25 \\
25-30 \\
30-35 \\
30-35 \\
35-40 \\
40-45\end{array}$ \\
\hline
\end{tabular}


Die Ermittlung des Spannungsintensitätsfaktors $K_{I}$ erfolgte, wie in Abschnitt 4.2 erläutert, innerhalb des Postprocessings von ANSYS mit Hilfe der Routine CINT entlang der jeweils vorhandenen 12 Konturen um die Rissspitze (siehe Abbildung 4.4). Angesichts der sehr feinen Vernetzung konvergierte die derart bestimmte Rissspitzenbeanspruchung bereits ab der 6 Kontur. Die Berechnung des Spannungsintensitätsfaktors erfolgte dabei immer im Bereich der größten Spannungsintensität in der Mitte der Rissfront.

Die Ergebnisse aller numerischen Berechnungen sind zusammen mit den entsprechenden Geometrieparametern in Stroetmann; Sieber et al. (2015) tabellarisch zusammengefasst. Der Geometriefaktor $Y_{F E M}$ ergibt sich als bezogener Spannungsintensitätsfaktor zu:

$$
Y_{F E M}=\frac{K_{I, F E M} \cdot b \cdot t}{P \cdot \sqrt{\pi a}}
$$

Für den Anschluss eines Winkelprofils L60x6 mit einem Verbindungsmittel und einseitigem Anriss der Länge $a=3 \mathrm{~mm}$ am Lochrand ist exemplarisch $Y_{F E M}$ in Abbildung 4.9 grafisch dargestellt. Wie erwartet wird deutlich, dass mit steigendem Durchmesser des Loches und sinkendem Lochabstand vom Rand, also schmaler werdendem Ligament, die Spannungsintensität steigt.

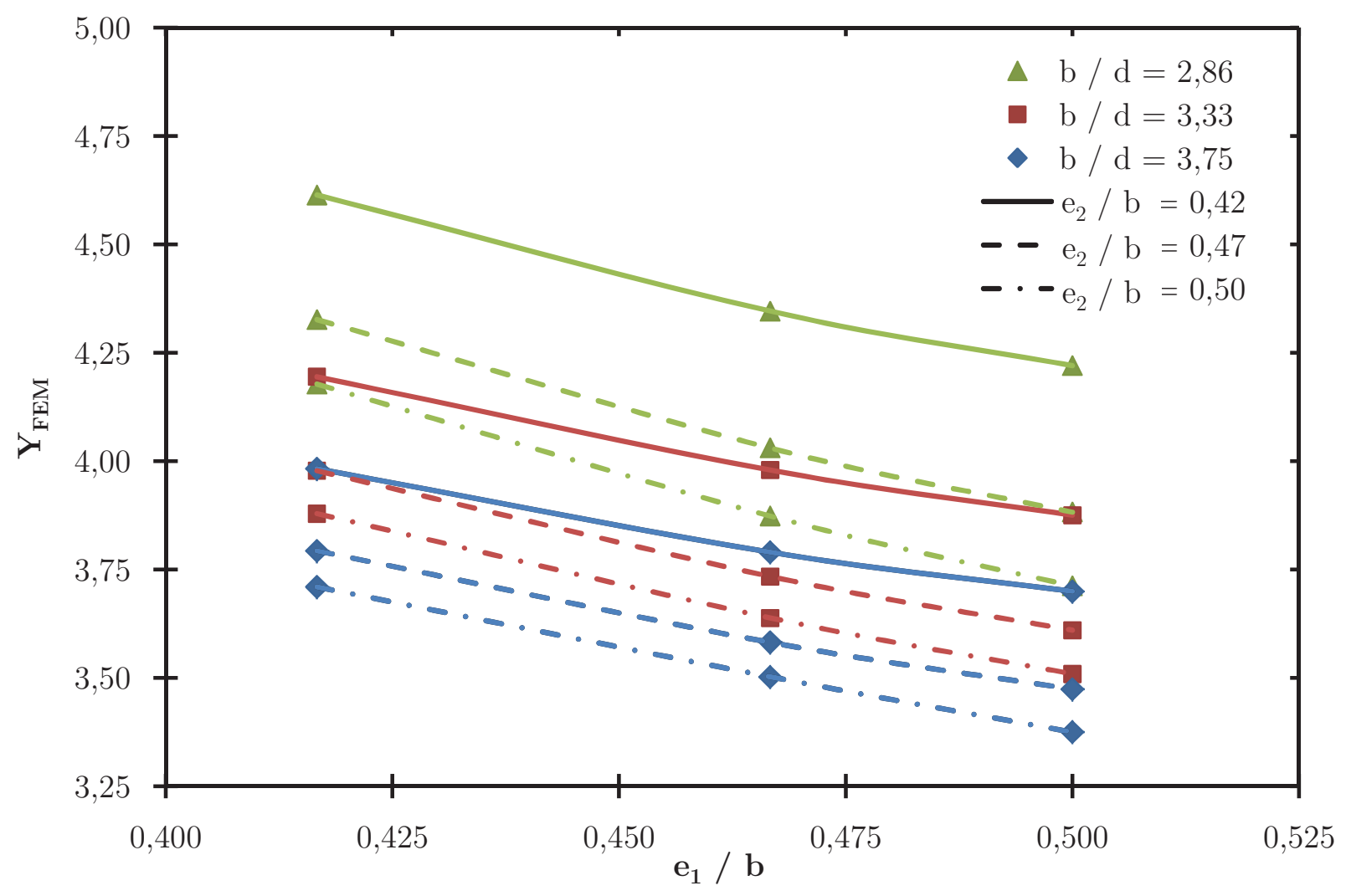

Abb. 4.9.: Bezogener Spannungsintensitätsfaktor für den Anschluss eines Winkels L60x6 mit einem Verbindungsmittel und $3 \mathrm{~mm}$ Anriss am Lochrand 


\subsection{Gegenüberstellung mit bekannten K-Lösungen aus der Fachliteratur}

\subsubsection{Winkelprofilanschlüsse mit einem Verbindungsmittel}

Um die in Abschnitt 4.3 mit der FE-Methode berechneten Ergebnisse des Spannungsintensitätsfaktors im untersuchten Parameterraum für weitere Berechnungen zur Verfügung zu stellen, werden die Ergebnisse üblicherweise durch geeignete mathematische Funktionen, in Abhängigkeit der Geometrieparameter, approximiert. Abweichend davon wird im Folgenden auf bekannte K-Faktor-Lösungen aus der Fachliteratur zurückgegriffen, die bereits in Abschnitt 2.6.2 ausführlich vorgestellt wurden. Für die Ermittlung der Rissspitzenbeanspruchung des in Abbildung 4.10 dargestellten Konstruktionsdetails eines Winkelprofilanschlusses mit einem Verbindungsmittel und einseitigem Durchriss, sind die Lösungen von TADA; PARIS et al. (2000) (Gleichung 2.16) oder NEWMAN (1971) (Gleichung 2.19) nicht geeignet, da sie nur den Fall des symmetrischen Anrisses am Lochrand abdecken. Darüber hinaus kann die Lasteinleitung am Loch aus der Kraftübertragung im Anschluss mit beiden Modellen nicht berücksichtigt werden.

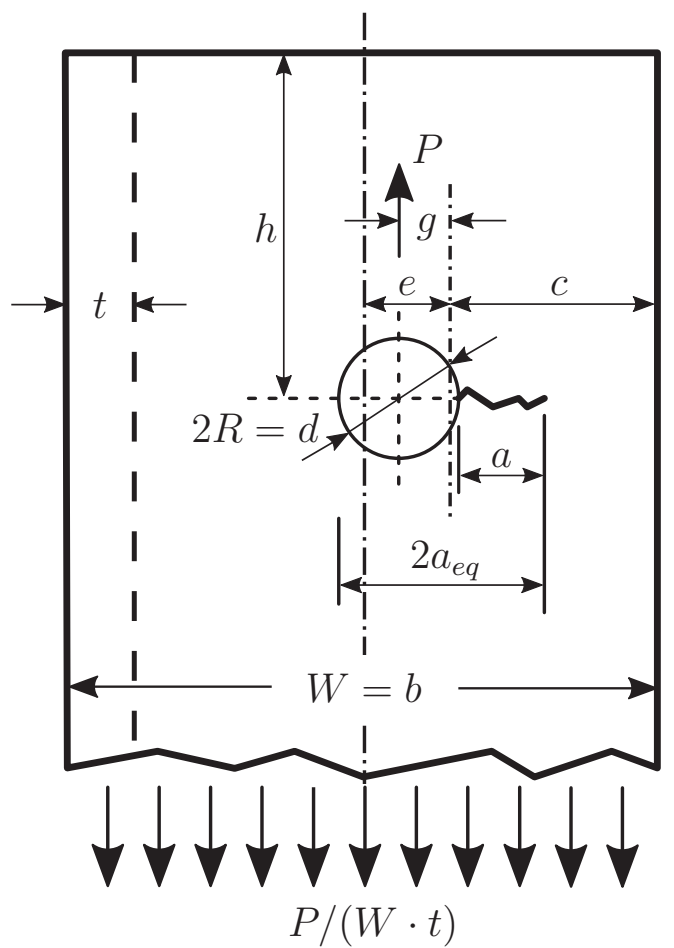

Abb. 4.10.: Geometriedefinition eines Winkelprofilanschlusses mit einem Verbindungsmittel ana$\log$ zu BALL (1987)

Ist der Verlauf der Zugspannungen in Rissachse aus einer Berechnung mittels FEM am ungerissenen Modell bekannt, kann die Rissspitzenbeanspruchung, für den Fall des einseitigen Durchrisses anhand der Gewichtsfunktionslösungen von Wu; CARLsson (1991) nach Gleichung 2.42, ermittelt werden. Hierfür wurden ergänzend zu den bruchmechanischen FEBerechnungen in Abschnitt 4.3 die gleichen Winkelprofilanschlüsse mit einem Verbindungsmittel ohne Anriss untersucht. Die Ergebnisse der Auswertung mittels Gewichtsfunktion 
sind, bezogen auf die der FEM-Analyse, in Abbildung 4.11 links dargestellt. Unabhängig von der Geometrie des Anschlusses wird die Rissspitzenbeanspruchung mit der Lösung nach Wu; Carlsson (1991) im Mittel um knapp 5\% unterschätzt. Grund hierfür ist die Modellannahme symmetrisch verlaufender Zugspannungen beiderseits des Loches. Aufgrund der exzentrischen Zugbeanspruchung des Winkelprofils sind die Spannungen am ungerissenen Lochrand jedoch kleiner und die Rissspitzenbeanspruchung am gerissenen Rand folglich größer. Die Ergebnisse der bruchmechanischen FE-Berechnungen und die Güte der Modellierung des Anrisses konnten auf diesem Wege validiert werden. Für die praktische Anwendung zur bruchmechanischen Analyse der Sprödbruchgefährdung genieteter Bauteile ist die Spannungsermittlung mittels FEM jedoch zu aufwendig.

Für die analytische Ermittlung der Rissspitzenbeanspruchung an einem Anschluss mit nur einem Verbindungsmittel eignet sich dagegen die Lösung von Forman; SHIVAKumar et al. (2002) nach Gleichung 2.29. Diese wird u. a. in der bruchmechanischen Berechnungssoftware NASGRO zur Analyse von Laschen und Ösen angewendet. Die Ergebnisse für die hier untersuchten Winkelprofilanschlüsse mit Anriss am Loch sind in Abbildung 4.11 links, bezogen auf die Ergebnisse der FEM-Analyse, dargestellt. Es wird deutlich, dass die Lösung von Forman; Shivakumar et al. (2002) die Rissspitzenbeanspruchung in den Winkelprofilen um 20 bis $55 \%$ überschätzt. Der Unterschied wächst mit steigenden Randabständen $c$ orthogonal und $h$ parallel zur Kraftrichtung. Bei Vergrößerung des Lochdurchmessers und Minimierung der Abstände vom Bauteilrand nähert sich die Lösung den numerischen Ergebnissen. Die Überschätzung der Beanspruchung am Riss ist in der Herleitung dieser Lösung begründet. Sie wurde abgeleitet für Laschen bzw. Ösen (siehe Abbildung 2.22) mit rundem Kopf. Diese zeigen unter der Bolzenlast eine deutlich größere Verformung und Rissöffnung und damit auch eine höhere Rissspitzenbeanspruchung als bei einem rechteckigen Laschenkopf. Diese Lösung sollte folglich nur für Anschlüsse mit ähnlich abgerundetem Bauteilende und annähernd gleichen Randabständen des Loches angewendet werden.

Die beste Übereinstimmung mit den Ergebnissen der numerischen Analyse zeigt die Lösung von BALL (1987), wie die Gegenüberstellung in Abbildung 4.11 rechts mit einer Überschätzung von maximal $10 \%$ bestätigt. Entsprechend der Superposition der Rissspitzenbeanspruchungen, infolge Lasteinleitung und Zugbeanspruchung im Bauteil in Gleichung 2.25, ergibt sich durch Einsetzen von Gleichung 2.20 und 2.26 und Zusammenfassen der Geometriefaktoren:

$$
K_{I}=\frac{P}{2 \cdot W_{e f f} \cdot t} \cdot \sqrt{\pi a} \cdot \beta_{F}
$$

mit $W_{\text {eff }}=2 c$ und dem Geometriefaktor $\beta_{F}$ für den Anschluss mit einem Verbindungsmittel (Fastener):

$$
\beta_{F}=\frac{W_{e f f}}{\sqrt{2 \pi a c}} \cdot F_{P} \cdot F_{H P W}+\left(F_{F W}+2 \cdot F_{E C C}\right) \cdot F_{T H}
$$

Um den Faktor $F_{H P W}$, der bei BALL (1987) nur grafisch als Diagramm angegeben ist, für eine Programmierung der Lösung nutzbar zu machen, wurde dieser im Parameterbereich der untersuchten Winkelprofilanschlüsse für $0,25 \leq 2 a_{e q} / W \leq 0,5$ und $0,25 \leq h / W \leq 0,5$ mit folgender Gleichung approximiert.

$$
F_{H P W}=\left[23,76\left(\frac{h}{W}\right)^{2}-28,558\left(\frac{h}{W}\right)+8,913\right] \cdot\left(\frac{2 a_{e q}}{W}\right)+1,284\left(\frac{h}{W}\right)+0,322
$$



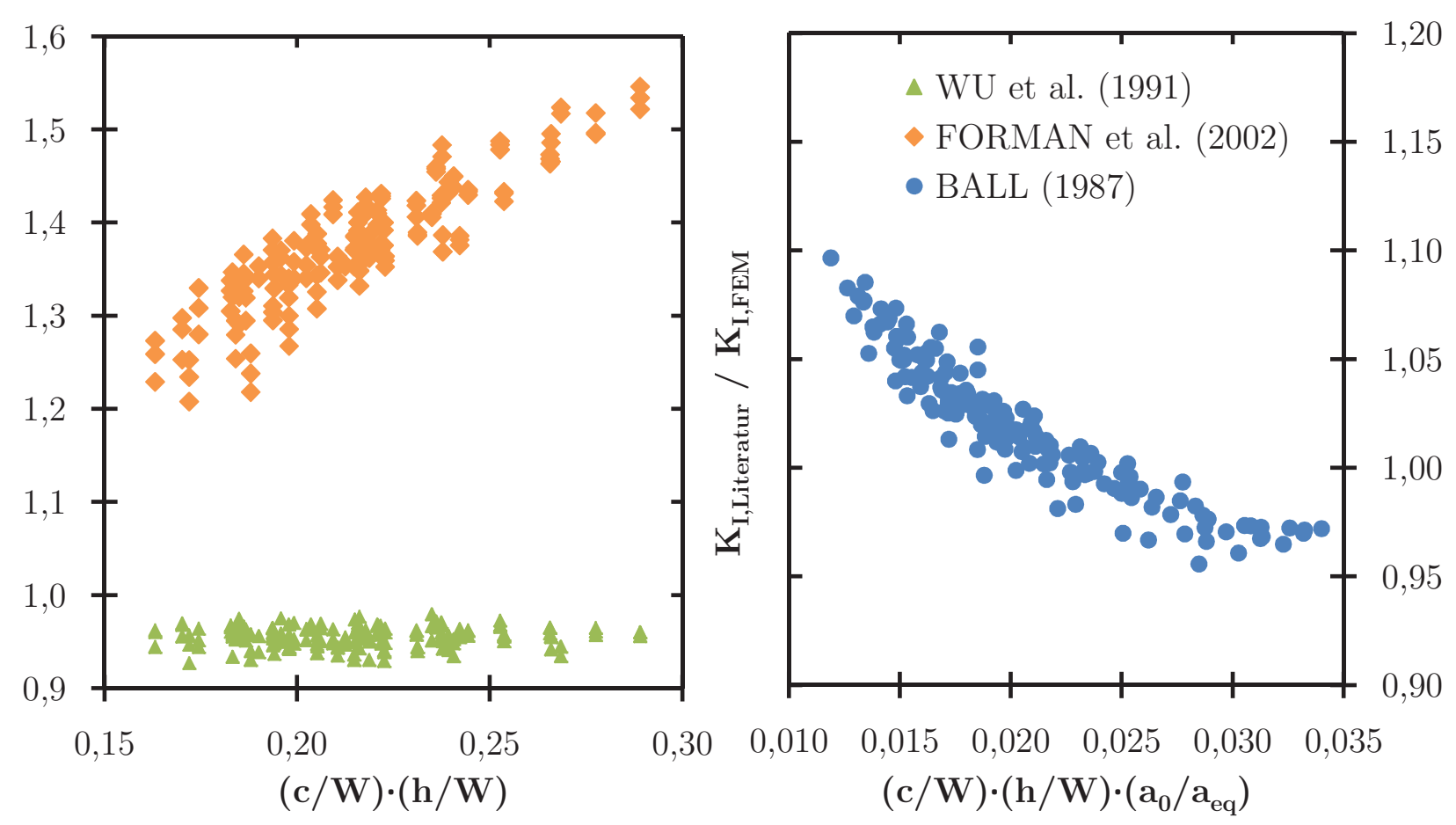

Abb. 4.11.: Gegenüberstellung der FE-Berechnungsergebnisse mit K-Faktor-Lösungen für Lochstäbe aus der Fachliteratur

Der Faktor $F_{H P W}$ zur Berücksichtigung einer endlichen Bauteillänge wurde für den Fall einer Einzellast am Riss, jedoch ohne Lochschwächung hergeleitet (siehe Abbildung 2.21). Das tatsächlich vorhandene Nietloch mit Anriss schwächt den rückwärtigen Bereich $h$ des Anschlusses und führt zu einer Vergrößerung der Rissspitzenbeanspruchung. Um diese Schwächung berücksichtigen zu können, wurde anhand der Ergebnisse der numerischen Untersuchungen der effektive Randabstand $h_{\text {eff }}$ in Abhängigkeit des Lochdurchmessers $d$ abgeleitet.

$$
h_{e f f}=h-\frac{d}{3} \cdot \frac{(h+d) / 2}{2 a_{e q}}
$$

Ermittelt man aus den mit Gleichung 4.2 berechneten Spannungsintensitäten, analog zu Gleichung 4.1, den bezogenen Spannungsintensitätsfaktor $Y_{\text {Calc }}$ und stellt diesen den FEMErgebnissen aus Abschnitt 4.3 gegenüber (volle Datenpunkte in Abbildung 4.12), ist zu erkennen, dass die Lösungen von BALL (1987) die Ergebnisse der Winkelprofilanschlüsse bereits sehr gut beschreibt. Während bei kleinen Winkelprofilen die Rissspitzenbeanspruchung aus der FEM-Analyse um bis zu $5 \%$ unterschätzt wird, ergibt sich bei größeren Winkelprofilen die bereits erwähnte Überschätzung von bis zu 10\%. Der Grund dafür liegt vor allem in der Vernachlässigung der Versteifung durch den zweiten, abstehenden Winkelschenkel, der zu einer Entlastung des Risses auf der gegenüberliegenden Seite des Nietloches führt. Um diesen Einfluss berücksichtigen zu können, wurde anhand der Ergebnisse der FEM-Analysen der Faktor $F_{L}$ abgeleitet.

$$
F_{L}=1,113-0,00252 \cdot \frac{W^{2}}{c \cdot h} \cdot \frac{a_{e q}}{a_{0}} \quad \text { mit: } a_{0}=1 \mathrm{~mm}
$$

Die Ergebnisse, unter Berücksichtigung dieses Anpassungsfaktors, sind in Abbildung 4.12 dargestellt. Die Abweichungen gegenüber den FEM-Ergebnissen betragen damit weniger als 
$3 \%$. Bei der Anwendung ist zu berücksichtigen, dass die Gültigkeit der vorangegangenen Gleichungen nur im untersuchten Parameterbereich der Winkelprofilquerschnitte (siehe Tabelle 4.1) und für eine konstante Risslänge von $a=3 \mathrm{~mm}$ überprüft und validiert wurde.

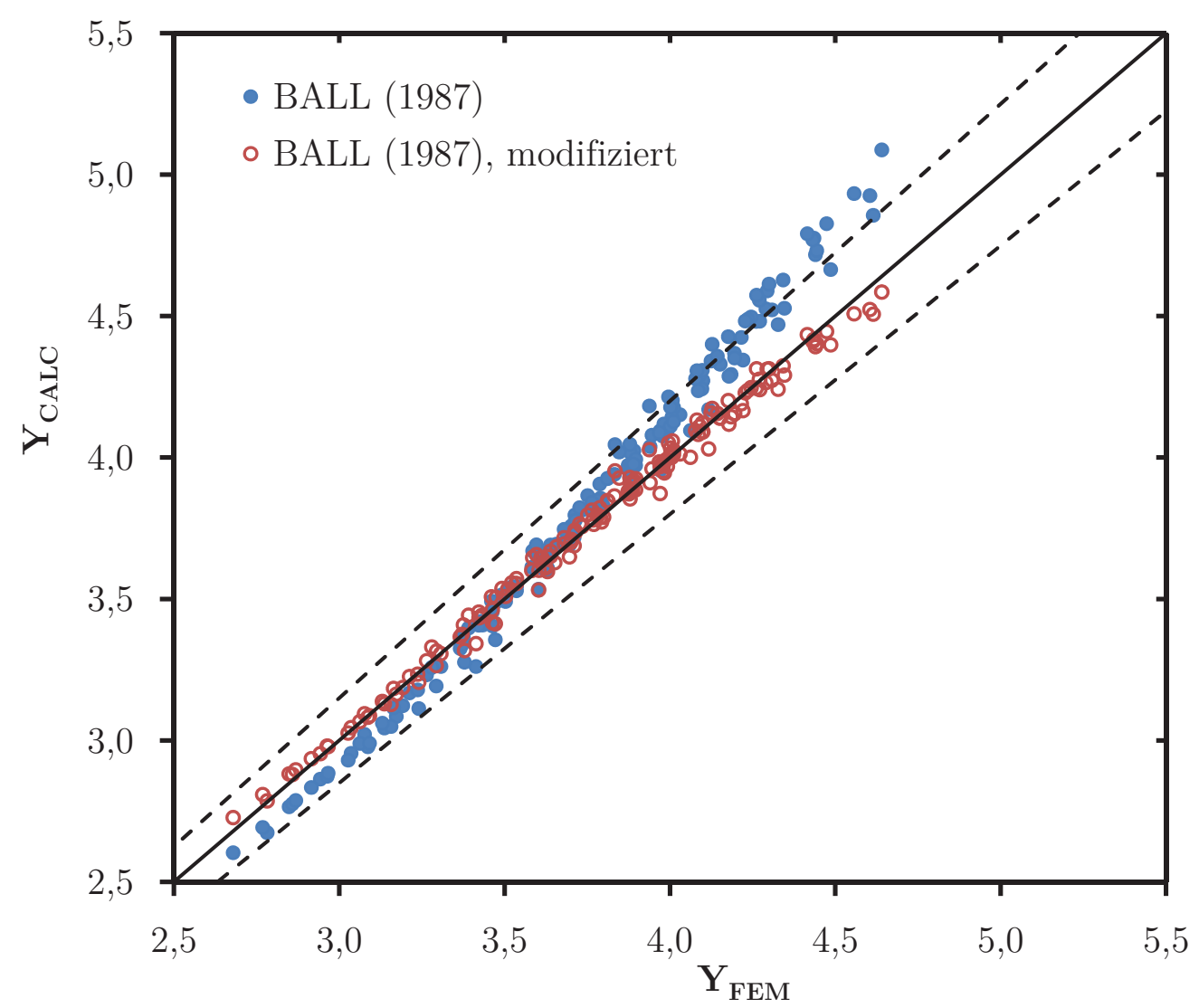

Abb. 4.12.: Gegenüberstellung der Spannungsintensität nach BALL (1987) mit denen aus der FEM-Analyse

\subsubsection{Winkelprofilanschlüsse mit mehreren Verbindungsmitteln}

Analog zur Berechnung von Anschlüssen mit einem Verbindungsmittel wird im Folgenden eine Lösung für Winkelprofilanschlüsse mit mehreren Verbindungsmitteln unter zentrischer Zugbeanspruchung abgeleitet. Die Geometrie und Belastung eines solchen Anschlusses ist in Abbildung 4.13 dargestellt.

Unter der Annahme annähernd gleicher Rand- und Lochabstände aller Verbindungsmittel und einer versetzten Anordnung der Löcher in beiden Schenkeln (siehe Abbildung 4.13), ist ein Anriss am Lochrand im Bereich maximaler Zugbeanspruchung zu erwarten. Folglich wird ein beidseitig symmetrischer Riss, am von der Bauteilmitte aus betrachtet ersten Loch, angenommen. Abhängig von der Anzahl der Verbindungsmittel wird ein Teil $P_{F}$ der Gesamtkraft $P_{\text {total }}$ im Bauteil an der Lochwandung dieses Loches eingeleitet (Fastener load) und der verbleibende Teil $P_{B P}$ der Zugkraft um das Loch herum geleitet (Bypass load). Damit ergibt sich die Spannungsintensität als Summe dieser beiden Beanspruchungen zu:

$$
K_{I}=\frac{P_{F}}{W_{e f f} \cdot t} \cdot \sqrt{\pi a} \cdot \beta_{P}+\frac{P_{B P}}{W_{L} \cdot t} \cdot \sqrt{\pi a} \cdot \beta_{R}
$$




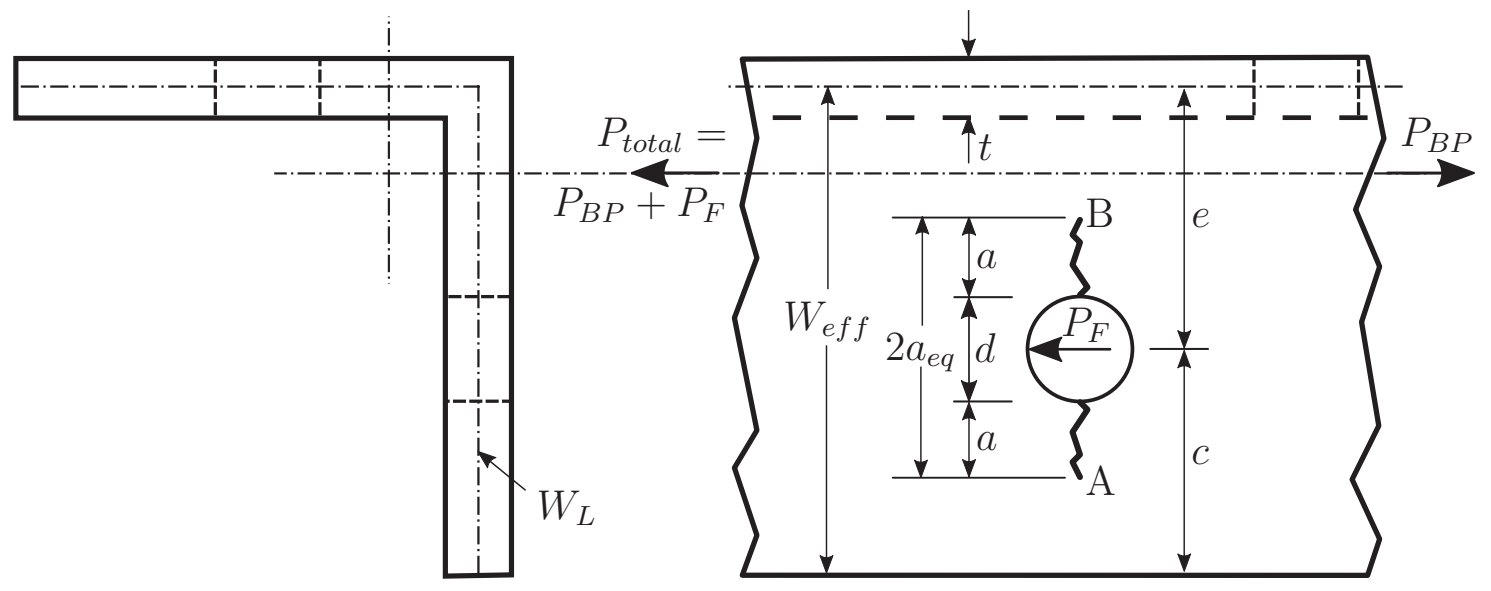

Abb. 4.13.: Geometriedefinition des Anschlusses eines Winkelprofils mit mehreren Verbindungsmitteln analog zu BALL (1987)

Der Geometriebeiwert $\beta_{P}$, für die Einleitung der Last $P_{F}$, wird analog zum Winkelanschluss mit einem Niet, jedoch ohne den Faktor $F_{H P W}$ berechnet, da das Bauteilende ausreichend weit entfernt ist und keinen Einfluss auf die Rissspitzenbeanspruchung hat.

$$
\beta_{P}=\frac{1}{2}\left[\frac{W_{e f f}}{\sqrt{2 \pi a c}} \cdot F_{P}+\left(F_{F W}+2 \cdot F_{E C C}\right) \cdot F_{T H}\right]
$$

Der Geometriebeiwert $\beta_{R}$ für die Durchleitung der Last $P_{B P}$ ergibt sich ebenso analog zu:

$$
\beta_{R}=\left(F_{F W}+F_{E C C}\right) \cdot F_{T H}
$$

Beschreibt man den Anteil der am Loch einzuleitenden Last zur Gesamtlast mit:

$$
\eta=\frac{P_{F}}{P_{\text {total }}}
$$

können $P_{F}$ und $P_{B P}$ wie folgt als Anteile der Gesamtlast $P_{\text {total }}$ dargestellt werden:

$$
\begin{array}{r}
P_{F}=\eta \cdot P_{t o t a l} \\
P_{B P}=(1-\eta) \cdot P_{t o t a l}
\end{array}
$$

Daraus folgt für die Spannungsintensität am beidseitig symmetrischen Anriss an der Stelle des höchst beanspruchten Querschnitts:

$$
K_{I}=\frac{P_{t o t a l}}{W_{L} \cdot t} \cdot \sqrt{\pi a} \cdot \beta_{\text {total }}
$$

mit dem Geometriefaktor für den Anschluss mit mehreren Verbindungsmitteln:

$$
\beta_{\text {total }}=\eta \cdot\left[\frac{W_{L}}{\sqrt{8 \pi a c}} \cdot F_{P}+\left(F_{F W}+2 \cdot F_{E C C}\right) \cdot F_{T H}\right]+(1-\eta) \cdot\left(F_{F W}+F_{E C C}\right) \cdot F_{T H}
$$

Unter der Voraussetzung üblicher Lochabstände von $3 d$ bis $4 d$ in Kraftrichtung [GÖBEL; HENKEL (1921)], wie auch bei mehrreihiger Vernietung zu benachbarten Löchern [BLEICH (1933)], kann die Kerbwirkung dieser Löcher auf die Rissspitzenbeanspruchung vernachlässigt werden. Entsprechende Geometriefaktoren für Gruppen von Löchern bzw. Rissen, wie sie z. B. bei MURAKAMI (1990) angegebenen sind, betragen bei diesen Lochabständen annähernd 1,0. 



\section{Kapitel 5.}

\section{Bruchmechanischer Sicherheitsnachweis}

\subsection{Allgemeines}

Aufbauend auf den Ergebnissen der numerischen Untersuchungen in Kapitel 4 werden in diesem Kapitel Bemessungsmodelle abgeleitet, die zur Ermittlung der Grenztragfähigkeit von Winkelprofilanschlüssen, unter Vermeidung spröden Bauteilversagens, angewendet werden können. Wesentliche Grundlage bildet dabei die in Abschnitt 3.4.3 ermittelte Bruchzähigkeit alter Flussstähle. Die Validierung der Bemessungsmodelle erfolgt mit Hilfe der in Abschnitt 3.6 beschriebenen Bauteilzugversuche. Durch Gegenüberstellung der rechnerischen Grenzlasten mit den experimentell ermittelten Versagenslasten und Auswertung nach DIN EN 1990 werden anschließend die für die Modelle erforderlichen Teilsicherheitsbeiwerte ermittelt.

\subsection{Statistische Auswertung nach DIN EN 1990, Anhang D}

Ein mögliches Verfahren zur statistischen Auswertung und Anpassung eines Widerstandsmodells und der Bestimmung von Bemessungswerten auf Basis experimenteller Daten ist im Anhang D von DIN EN 1990:2010 angegeben. Ausgehend von theoretischen Überlegungen und entsprechenden Bauteilversuchen wird zunächst ein Bemessungsmodell entwickelt. Dieses soll durch statistische Auswertung der Versuchsergebnisse überprüft und ggf. so modifiziert werden, dass eine ausreichende Korrelation zwischen theoretischen und experimentellen Werten erreicht wird. Die in DIN EN 1990 standardisierte Vorgehensweise ist im Folgenden schrittweise erläutert.

In einem ersten Schritt wird das entwickelte Bemessungsmodell in Form einer oder mehrerer Widerstandsfunktionen

$$
r_{t}=g_{r t}(\underline{X})
$$

ausgedrückt. Diese Funktionen müssen alle Basisvariablen $\underline{X}$ (Abmessungen, Querschnittswerte, Werkstoffkennwerte, etc.) beinhalten, die den betrachteten Grenzzustand maßgeblich beeinflussen. Diese Variablen sind als Grundlage für die folgende Auswertung für jeden Prüfkörper zu bestimmen. 
Im zweiten Schritt werden durch Einsetzen der gemessenen Werte in die Widerstandsfunktion $r_{t}$ die theoretischen Werte $r_{t i}$ ermittelt und in einem Diagramm den experimentellen Werten $r_{e i}$ gegenübergestellt (vgl. Abbildung 5.3). Beschreibt die Widerstandsfunktion den betrachteten Grenzzustand exakt und vollständig, würden alle Wertepaare auf einer Geraden liegen. Aufgrund von Streuungen in der Praxis gibt es Abweichungen, die im dritten und vierten Schritt durch die Mittelwertkorrektur $b$ und das Streumaß $\delta_{i}$ sowie den daraus abgeleiteten Variationskoeffizienten $V_{\delta}$ ausgedrückt werden.

In Schritt 5 wird eine Verträglichkeitsprüfung der Widerstandsfunktion anhand der Versuchsergebnisse gefordert, in deren Ergebnis eine Anpassung der Funktion vorgenommen werden kann, z.B. durch Berücksichtigung weiterer Parameter oder Auswertung der Versuche nach Untergruppen.

Im anschließenden Schritt 6 sind die Variationskoeffizienten $V_{X i}$ der Basisvariablen zu ermitteln. Diese basieren entweder auf vorhandenen Vorinformationen (z.B. JCSS (2001a)), auf eigenen statistischen Auswertungen oder sind als konservative Schätzwerte anzunehmen.

Im abschließenden Schritt 7 werden die charakteristischen Werte $r_{k}$ und die Bemessungswerte $r_{d}$ der Widerstandsfunktion ermittelt. Grundlage dafür bildet der Variationskoeffizient der Widerstandsfunktion $V_{r t}$, der mit Hilfe der partiellen Ableitungen nach den Basisvariablen wie folgt ermittelt wird:

$$
V_{r t}^{2}=\frac{1}{g_{r t}{ }^{2}\left(\underline{X}_{m}\right)} \cdot \sum_{i=1}^{k}\left(\frac{\delta g_{r t}\left(X_{i}\right)}{\delta X_{i}} \cdot \sigma_{i}\right)^{2}
$$

Im Falle einer komplexen Widerstandsfunktion können diese partiellen Ableitungen nur numerisch und für jeden Prüfkörper separat berechnet werden. Dafür wird von der Empfehlung von Silva, da; TAnkova et al. (2015) Gebrauch gemacht, in dem unter Ansatz eines sehr kleinen Wertes $\Delta X_{i}$ die Ableitung in einen Differenzenquotienten überführt wird.

$$
\frac{\delta g_{r t}\left(X_{i}\right)}{\delta X_{i}} \approx \frac{g_{r t}\left(X_{1}, \ldots, X_{i}+\Delta X_{i}, \ldots, X_{k}\right)-g_{r t}\left(X_{1}, \ldots, X_{i}, \ldots, X_{k}\right)}{\Delta X_{i}}
$$

Da die Anzahl der auswertbaren Versuche kleiner als 100 und damit begrenzt ist, wird die statistische Unsicherheit über Fraktilen- und Wichtungsfaktoren auf der Basis einer Studentverteilung berücksichtigt.

Aus dem Quotienten von charakteristischem Wert zum Bemessungswert resultiert schlussendlich der Teilsicherheitsbeiwert $\gamma_{M}$. Anschließend erfolgt die Anpassung des Bemessungsmodells unter Ansatz von Nennwerten, also z.B. charakteristischen Materialkennwerten. Hierfür wird der korrigierte Teilsicherheitsbeiwert $\gamma_{M}{ }^{*}$ der Widerstandsfunktion ermittelt.

Das beschriebene Vorgehen zur Validierung und Anpassung des Widerstandsmodells anhand von Versuchsergebnissen wurde für die untersuchten Konstruktionsdetails in den folgenden Abschnitten angewendet. Dabei wird zwischen Winkelprofilanschlüssen mit einem und mehreren Verbindungsmitteln unterschieden. 


\subsection{Bemessungsmodelle zur Bestimmung der Grenzlast von Winkelprofilanschlüssen}

\subsubsection{Grenzlast für Winkelprofilanschlüsse mit einem Verbindungsmittel}

Wie bereits in Abschnitt 2.6.3 erläutert, existieren in den aktuellen Bewertungsvorschriften sehr ähnliche Methoden für die bruchmechanische Bauteilbewertung anrissgefährdeter Bauteile. Da für die in dieser Arbeit untersuchten Flussstähle die Streckgrenze und die Zugfestigkeit bekannt sind, erfolgt die Bewertung auf der Analyse-Ebene 1A nach SINTAP für einen Werkstoff mit Lüdersplateau. Die Grenzkurve des Failure Assessment Diagrams (FAD) ist in Abbildung 2.25 bereits angegeben.

Aus dem zum FAD gleichwertigen Crack Driving Force-Ansatz (CDF)

$$
J=J_{\text {elast }} \cdot\left[f\left(L_{r}\right)\right]^{-2} \leq J_{\text {mat }}
$$

und der im ebenen Spannungszustand gültigen Umrechnung [SchwalBE (1980)]

$$
J=\frac{K_{I}^{2}}{E}
$$

ergibt sich die Forderung

$$
\frac{K_{I}}{f\left(L_{r}\right)} \leq K_{m a t}=K_{J c}
$$

Durch Umstellen von $f\left(L_{r}\right)$ nach Gleichung 2.50 und Einsetzen von $K_{I}$ nach Gleichung 4.2 mit $N=P$ sowie $L_{r}=N / F_{Y}$ ergibt sich für den Anschluss des Winkelprofils mit einem Verbindungsmittel die Grenztragfähigkeit

$$
N_{\text {grenz }}=\sqrt{\sqrt{F_{Y}^{4}+\left(\frac{\sqrt{8} \cdot F_{Y} \cdot K_{m a t} \cdot W_{e f f} \cdot t}{\sqrt{\pi a} \cdot \beta_{F}}\right)^{2}}-F_{Y}^{2}}
$$

Ausgehend von der Definition des FAD gilt als oberer Grenzwert für $N_{\text {grenz }}$ die plastische Grenzlast $F_{Y}$. Diese wird für den einschenkligen Anschluss analog zu den Herleitungen von EHRHARDT (1988) für Proben mit symmetrischem Innenriss (CCT) mit

$$
F_{Y}=\left(W-2 a_{e q}\right) \cdot t \cdot R_{e L}
$$

festgelegt (siehe auch Abschnitt 2.6.4). Dieser Ansatz entspricht anschaulich einer Plastizierung des Nettoquerschnitts des angeschlossenen Winkelschenkels mit Anriss am Lochrand.

In Abbildung 5.1 sind die Ergebnisse der Bauteilzugversuche an Winkelprofilen mit Anschlüssen durch ein Verbindungsmittel und seitlichem Riss am Lochrand im FAD dargestellt. Ausgehend von den Herleitungen in Abschnitt 4.4.1 wurde für jeden Prüfkörper das Versagen mit einseitigem Anriss $(n=1)$ und beidseitig symmetrischem Anriss $(n=2)$ untersucht. Die Berechnungen erfolgten jeweils mit den tatsächlich gemessenen Größen, d. h. der realen Anschlussgeometrie und der im Zugversuch ermittelten Streckgrenze $R_{e L}$. Eine Ermittlung der tatsächlichen Bruchzähigkeit $K_{J c}$ für jeden Prüfkörper war aufgrund des erheblichen experimentellen Aufwandes nicht möglich. Hier wurde der Mittelwert $K_{J c, 50 \%}$ der multimodalen Master-Curve-Auswertung für Flussstähle nach Abschnitt 3.4.3 angesetzt. 
Bei der Validierung des Bemessungsmodells für die bruchmechanische Bauteilbewertung mit Hilfe von Bauteilzugversuchen ist zu beachten, dass sich die Sicherheiten im FAD umkehren. Im Failure Assessment Diagram gilt ein Bauteil üblicherweise als sicher (siehe auch Abschnitt 2.6.3), wenn der Bewertungspunkt innerhalb des durch die Schadensgrenzkurve begrenzten sicheren Bereichs liegt. Je größer der Abstand des Bewertungspunktes von der Grenzkurve ist, umso größer ist die Sicherheitsreserve. Bei der Nachrechnung von Versuchen wechselt diese Betrachtungsweise. Nur wenn der Bewertungspunkt außerhalb der Schadensgrenzkurve liegt, ist die durch das Nachweismodell beschriebene Grenzlast kleiner als die experimentell ermittelte Versagenslast und die Anwendung des Modells führt zu einem Bauteil mit Sicherheitsreserve. Die im Nachweisverfahren enthaltenen Sicherheiten nehmen folglich zu, je weiter die Bewertungspunkte der Nachrechnungen von der Grenzkurve entfernt sind.

Die Ergebnisse der Bauteilversuche in Abbildung 5.1 zeigen somit eine gute Übereinstimmung mit dem Bemessungsmodell. Alle Bewertungspunkte liegen nahe der Schadensgrenzkurve. Nahezu alle Punkte liegen außerhalb des von der Grenzkurve eingeschlossenen, sicheren Bereichs, nur wenige knapp innerhalb. Der Fall des einseitigen Anrisses am Lochrand ist maßgebend. Die Güte der Ergebnisse ist qualitativ mit denen in Untersuchungen von VERSCH (2014) vergleichbar, wo ebenfalls mit Erwartungswerten der Werkstoff- und Bauteilkenngrößen gearbeitet wurde.

Die in den Versuchen eingetretenen Bruchbilder eines makroskopisch überwiegend spröden Versagens im Übergang zu teilplastizierten Querschnittsbereichen sind auch in der FADAuswertung in Abbildung $5.1 \mathrm{zu}$ erkennen. Ebenso wie sich das Grundmaterial Flussstahl gemäß der Master-Curve-Auswertung in Abschnitt 3.4.3 bei der Versuchstemperatur bereits

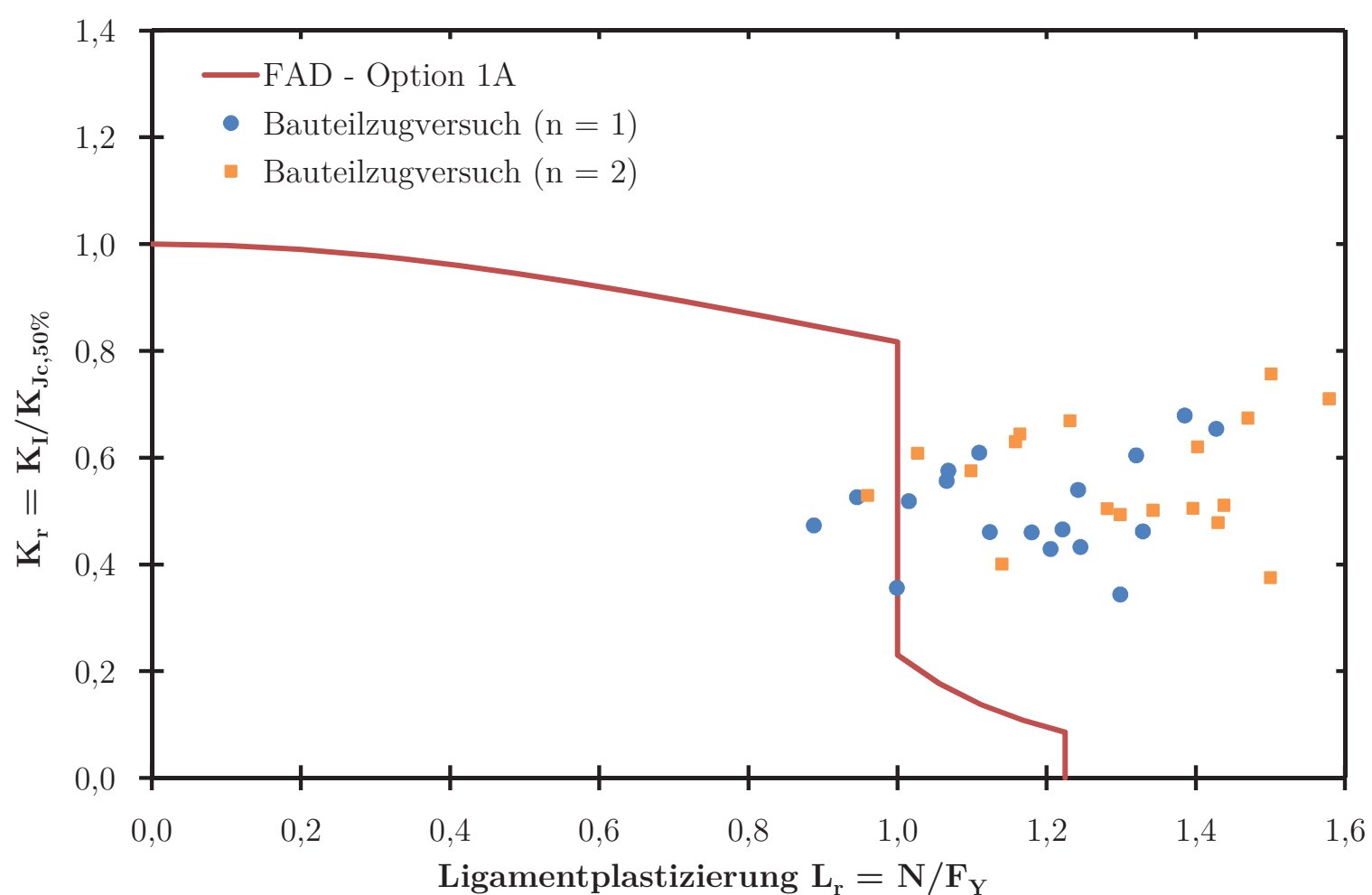

Abb. 5.1.: Failure Assessment Diagram mit den Ergebnissen der Bauteilzugversuche an Winkelprofilen mit Anschlüssen durch ein Verbindungsmittel 
im unteren Übergangsbereich befindet, liegen die Versuchsergebnisse im FAD im Übergang vom spröden zum duktilen Versagen.

\subsubsection{Grenzlast für Winkelprofilanschlüsse mit mehreren Verbindungsmitteln}

Analog zur Herleitung in Abschnitt 5.3.1, unter Berücksichtigung der maßgebenden Spannungsintensität $K_{I}$ am Winkelprofilanschluss mit mehreren Verbindungsmitteln nach Gleichung 4.7, ergibt sich die Grenztragfähigkeit zu:

$$
N_{\text {grenz }}=\sqrt{\sqrt{F_{Y}^{4}+\left(\frac{\sqrt{2} \cdot F_{Y} \cdot K_{\text {mat }} \cdot W_{L} \cdot t}{\sqrt{\pi a} \cdot \beta_{\text {total }}}\right)^{2}}-F_{Y}^{2}}
$$

Der obere Grenzwert für $N_{\text {grenz }}$ entspricht ebenfalls der plastischen Grenzlast $F_{Y}$, die mit

$$
F_{Y}=\left(W_{L}-n_{R i s s} \cdot d-2 a\right) \cdot t \cdot R_{e L}
$$

ermittelt wird. Dieser Ansatz entspricht analog zu Gleichung 5.8 der Plastizierung des Nettoquerschnitts des angeschlossenen Winkels mit beidseitig symmetrischem Anriss am höchst beanspruchten Loch. Der Wert $n_{\text {Riss }}$ gibt die Anzahl der Nietlöcher an, die im Versagensfall auf der Risslinie liegen.

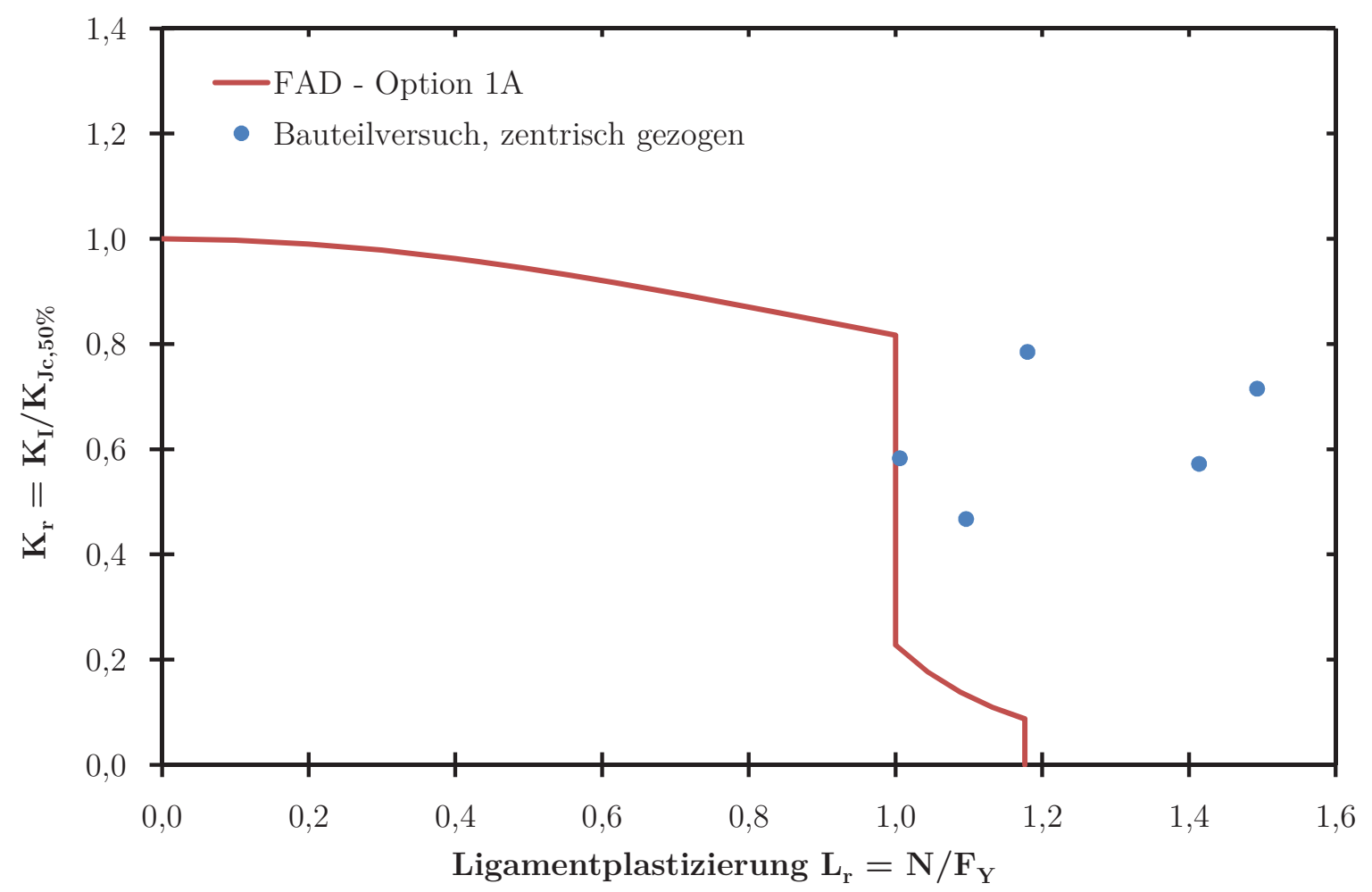

Abb. 5.2.: Failure Assessment Diagram mit den Ergebnissen der Bauteilzugversuche an Winkelprofilen mit Anschlüssen durch mehrere Verbindungsmittel 
In Abbildung 5.2 sind die Ergebnisse der Bauteilzugversuche an Winkelprofilen mit Anschlüssen aus mehreren Verbindungsmitteln und seitlichem, symmetrischen Riss am höchst beanspruchten Nietloch im FAD dargestellt. Die Ermittlung der rechnerischen Beanspruchung erfolgte auch hier mit den gemessenen Größen der Anschlussgeometrie und der im Zugversuch ermittelten Streckgrenze $R_{e L}$. Die Bruchzähigkeit $K_{J c}$ wurde erneut mit dem Mittelwert der multimodalen Master-Curve-Auswertung für Flussstähle nach Abschnitt 3.4.3 berücksichtigt.

Auch die Traglastversuche an Winkelprofilen mit Anschlüssen aus mehreren Verbindungsmitteln zeigen eine gute Übereinstimmung mit den Ergebnissen des Berechnungsmodells nach Abschnitt 4.4.2. Alle Versagenslasten werden durch das Modell konservativ beschrieben. Die Lage der Versuchsergebnisse im FAD und die Bruchbilder (vgl. Abschnitt 3.6) weisen ebenfalls auf ein Versagen im Übergang zum Zähbruch mit überwiegenden Spaltbruchanteilen hin.

\subsection{Bestimmung der Teilsicherheitsbeiwerte für die Bemessungsmodelle}

Die Auswertungen in Abschnitt 5.3 haben gezeigt, dass die in Bauteilzugversuchen ermittelten Traglasten von genieteten Bauteilen aus Flussstahl mit den hier entwickelten Bemessungsmodellen hinreichend genau beschrieben werden können. Dies wird auch noch einmal in der Gegenüberstellung der rechnerisch ermittelten Traglasten $N_{\text {Grenz }}$ und der experimentell bestimmten Bruchlasten $N_{E x p}$ in Abbildung 5.3 deutlich.

Darauf aufbauend wird die statistische Auswertung der Versuchsergebnisse nach DIN EN 1990, wie in Abschnitt 5.2 beschrieben, durchgeführt. Die Ergebnisse dieser Berechnungen

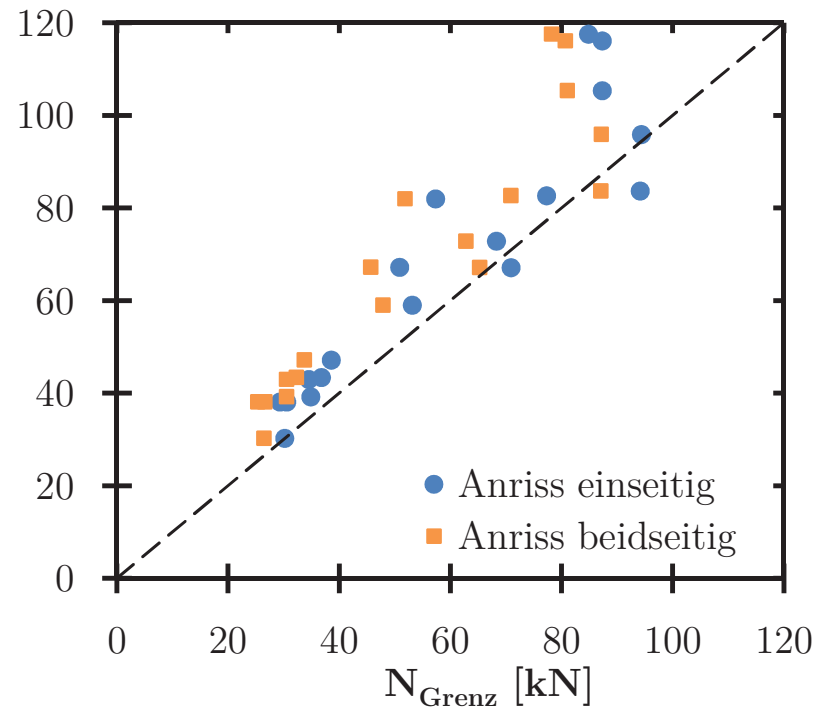

Winkelprofilanschlüsse mit einem Verbindungsmittel

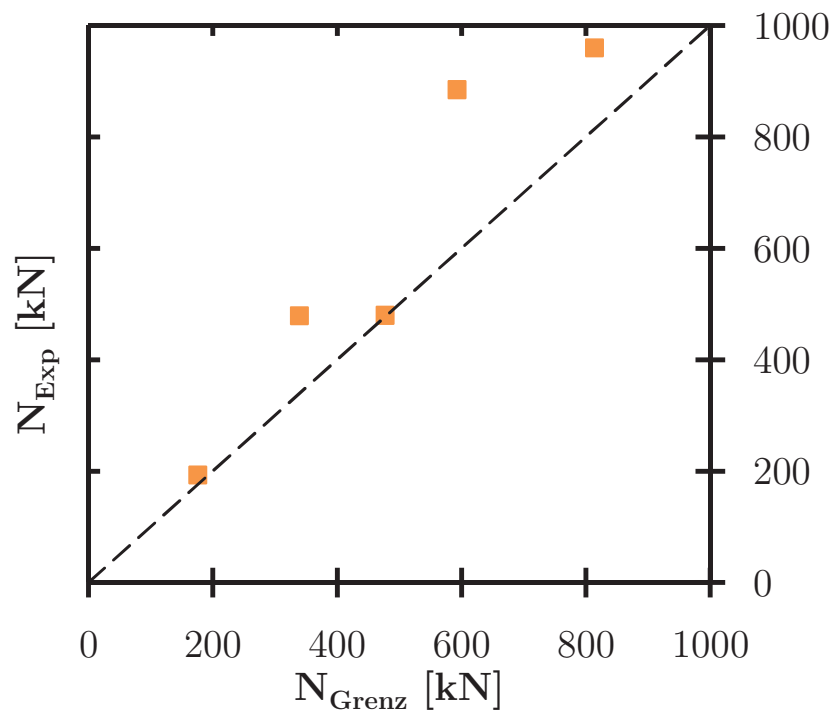

Winkelprofilanschlüsse mit mehreren Verbindungsmitteln

Abb. 5.3.: Gegenüberstellung der experimentell und rechnerisch ermittelten Traglasten 
sind jeweils für die Winkelprofilanschlüsse mit einem und mit mehreren Verbindungsmitteln in Tabelle 5.3 zusammengefasst.

Die in Abschnitt 5.3.1 und 5.3.2 hergeleiteten Widerstandsfunktionen beinhalten jeweils 7 Basisvariablen (Bauteil- und Anschlussgeometrie sowie Werkstoffkennwerte), die die Anschlusstragfähigkeit maßgebend beeinflussen. Die für die statistische Auswertung notwendigen Variationskoeffizienten dieser Variablen sind in Tabelle 5.1 angegeben. Die Streuungen der Bauteilabmessungen und der Anschlussgeometrie sind nicht mit denen heutiger Walzprodukte und Stahlkonstruktionen vergleichbar. Variationskoeffizienten für die geometrischen Größen, wie sie z.B. in JCSS (2001a) zur Verfügung gestellt werden, sind hier nicht anwendbar. Deshalb wurden im Rahmen dieser Arbeit für 155 Bauteile aus Fachwerk-Konstruktionen des Stahlhochbaus die Querschnittsabmessungen und die Anschlussgeometrie mit den Sollmaßen der Bauteile aus den Konstruktionszeichnungen verglichen und die Abweichungen statistisch ausgewertet. Die ermittelten Streuungen (siehe Tabelle 5.1) bestätigen die Ergebnisse von REICHE (2000), der die Abmessungen gewalzter Doppel-T-Profile untersuchte.

Auch die Streuung der Streckgrenze $R_{e L}$ alter Flussstähle ist nicht mit der heutiger Baustähle (siehe z.B. JCSS (2001b)) vergleichbar. Der hier verwendete Variationskoeffizient $V_{R_{e L}}$ basiert auf den statistischen Auswertungen von Niessen (1994) und LANGEnBerg (1996). Im Rahmen der Untersuchungen zur Bruchzähigkeit alter Flussstähle in Abschnitt 3.4.3 wurde deren Streuung nach dem Master-Curve-Konzept mit einer Weibull-Verteilung beschrieben. Der Exponent $m=4$ der Verteilungsfunktion verursacht eine Schiefe der Verteilungsdichtefunktion von annähernd Null [Spaethe (1992)]. Die verwendete Weibull-Verteilung entspricht damit näherungsweise einer logarithmischen Normalverteilung (siehe Abbildung 5.4). Der Variationskoeffizient des Master-Curve-Ansatzes kann folglich für die statistische Auswertung nach DIN EN 1990 verwendet werden.

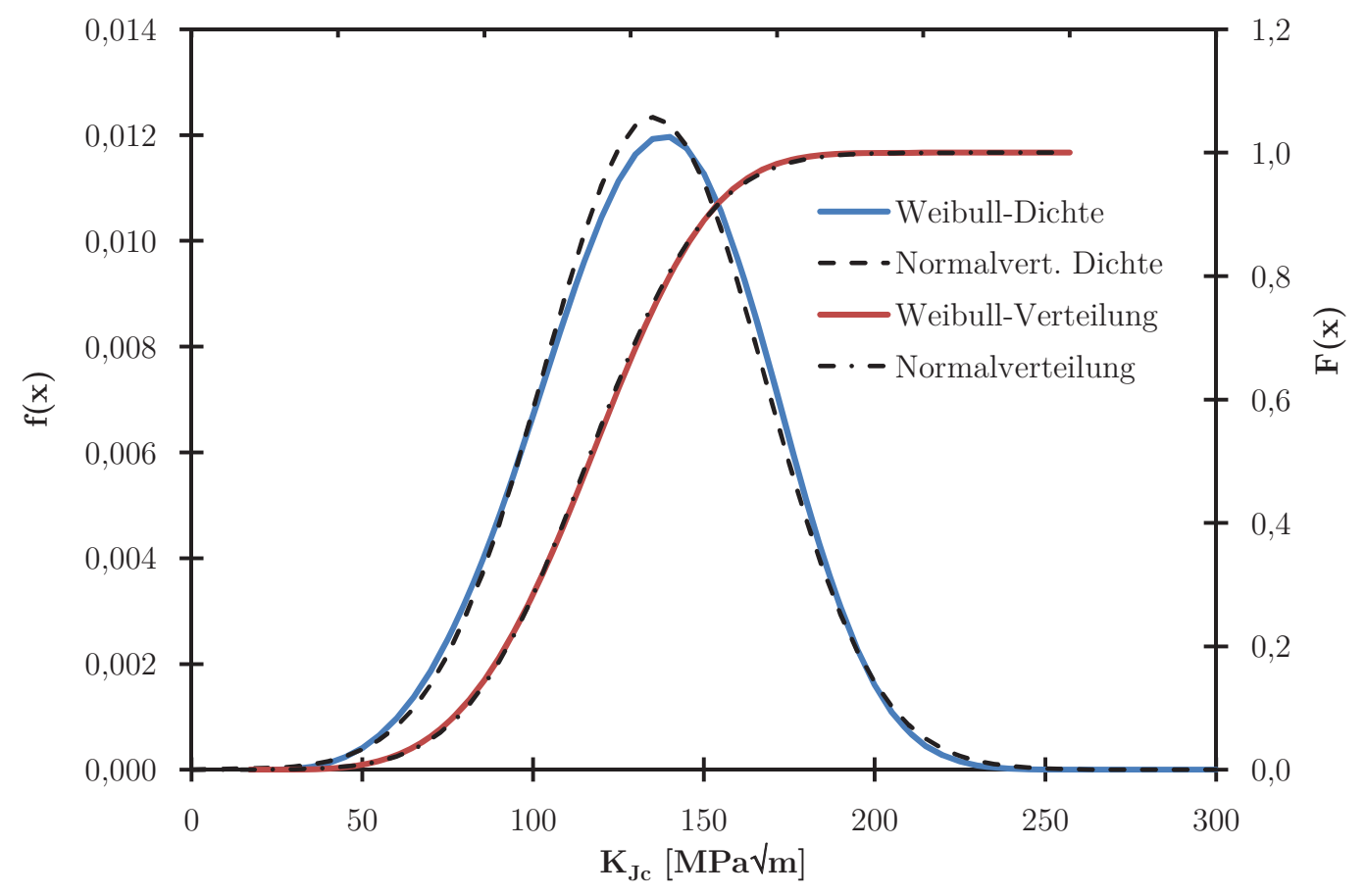

Abb. 5.4.: Gegenüberstellung der Weibull-Verteilung der Bruchzähigkeit im Master-CurveKonzept mit einer Normalverteilung 
Tab. 5.1.: Variationskoeffizienten $V_{X i}$ der Basisvariablen

\begin{tabular}{l|c|c}
\hline Basisvariable & $X_{i}$ & $V_{X i}$ \\
\hline Winkelschenkelbreite & $b$ & 0,02 \\
Winkelschenkeldicke & $t$ & 0,05 \\
Lochdurchmesser & $d$ & 0,02 \\
Lochabstände & $e_{1}, e_{2}, w_{1}, w_{2}$ & 0,07 \\
Streckgrenze & $R_{e L}$ & 0,12 \\
Bruchzähigkeit & $K_{J c}$ & 0,24 \\
\hline
\end{tabular}

Angesichts der Komplexität der Widerstandsfunktionen wird Gleichung 5.2, wie in Abschnitt 5.2 beschrieben, für jeden Versuch separat ausgewertet. Im weiteren Verlauf der statistischen Analyse wird der hieraus bestimmte Mittelwert von $V_{r t}$ verwendet. In Tabelle 5.2 sind die zur Berechnung der charakteristischen Werte $N_{G r, k}$ und der Bemessungswerte $N_{G r, d}$ notwendigen Fraktilenwerte nach Tabelle D.1 und D.2 in DIN EN 1990:2010 angegeben.

Tab. 5.2.: Fraktilenwerte nach DIN EN 1990, Tabelle D.1 und Tabelle D.2

\begin{tabular}{l|c|c}
\hline & $\begin{array}{c}\text { Anschlüsse mit einem } \\
\text { Verbindungsmittel }\end{array}$ & $\begin{array}{c}\text { Anschlüsse mit mehreren } \\
\text { Verbindungsmitteln }\end{array}$ \\
\hline Anzahl $n$ der Versuche & 18 & 5 \\
\hline$k_{n}$ & 1,688 & 1,800 \\
$k_{\infty}$ & 1,640 & 1,640 \\
$k_{d, n}$ & 3,174 & 3,370 \\
$k_{d, \infty}$ & 3,040 & 3,040 \\
\hline
\end{tabular}

Die Ergebnisse der statistischen Analyse zeigen, dass die auf bruchmechanischer Basis hergeleiteten Ansätze zur Bestimmung der Anschlusstragfähigkeit gut geeignet sind. Aufgrund der Streuungen der Basisvariablen und der Widerstandsfunktionen ergeben sich Variationskoeffizienten $V_{r}=0$,19 für die Anschlüsse mit einem Verbindungsmittel und $V_{r}=0,21$ für die Anschlüsse mit mehreren Verbindungsmitteln. Hieraus resultieren relativ niedrige Bemessungswerte $N_{G r, d}$ von $62,2 \%$ bzw. $56,5 \%$ der jeweiligen Widerstandsfunktionen sowie entsprechend hohe Teilsicherheitsbeiwerte $\gamma_{M}$ von 1,32 und 1,37. Dabei ist zu berücksichtigen, dass die deutliche Reduzierung der Tragfähigkeit der Anschlüsse mit mehreren Verbindungsmitteln, neben der Streuung der Eingangsgrößen, insbesondere auf die Streuung der Versuchsergebnisse sowie die geringe Anzahl der zur Verfügung stehenden Zugversuche zurückzuführen ist. Die Ergebnisse der statistischen Auswertung nach DIN EN 1990 sind in Abbildung 5.5 dargestellt (siehe auch Tabelle 5.3).

Zur Verwendung von Nennwerten der Basisvariablen in den Widerstandsfunktionen erfolgt die Bestimmung des Korrekturfaktors $k_{c}$ entsprechend DIN EN 1990, Anhang D nach folgender Gleichung:

$$
k_{c}=\frac{N_{g r}\left(\underline{X}_{k}\right)}{N_{g r}\left(\underline{X}_{m}\right)}
$$


Tab. 5.3.: Ergebnisse der statistischen Analyse nach DIN EN 1990, Anhang D.8

\begin{tabular}{|c|c|c|}
\hline & $\begin{array}{l}\text { Anschlüsse } \\
\text { mit einem } \\
\text { Verbindungs- } \\
\text { mittel }\end{array}$ & $\begin{array}{l}\text { Anschlüsse } \\
\text { mit mehreren } \\
\text { Verbindungs- } \\
\text { mitteln }\end{array}$ \\
\hline $\begin{array}{l}\text { Mittelwertkorrektur } b \\
b=\frac{\sum r_{e, i} \cdot r_{t, i}}{\sum r_{t, i}^{2}}\end{array}$ & 1,144 & 1,140 \\
\hline \multicolumn{3}{|l|}{ Variationskoeffizienten $V_{\delta}$ der Streugröße $\delta$} \\
\hline $\begin{array}{ll}\delta_{i} & =\frac{r_{e i}}{b \cdot r_{t i}} \\
\bar{\Delta} & =\frac{1}{n} \sum_{i=1}^{n} \Delta_{i}\end{array} \quad \Delta_{i}=\ln \left(\delta_{i}\right)$ & 0,014 & $-0,026$ \\
\hline$s_{\Delta}^{2}=\frac{1}{n-1} \sum_{i=1}^{n}\left(\Delta_{i}-\bar{\Delta}\right)^{2}$ & 0,018 & 0,027 \\
\hline$V_{\delta}=\sqrt{\exp \left(s_{\Delta^{2}}^{2}\right)-1}$ & 0,135 & 0,164 \\
\hline \multicolumn{3}{|l|}{ Charakteristischer Wert $r_{k}$ der Widerstandsfunktion } \\
\hline$V_{r t}=\sqrt{\frac{1}{g_{r t}^{2}\left(\underline{X}_{m}\right)} \cdot \sum_{i=1}^{k}\left(\frac{\delta g_{r t}\left(X_{i}\right)}{\delta X_{i}} \cdot \sigma_{i}\right)^{2}}$ & 0,135 & 0,132 \\
\hline$Q_{r t}=\sqrt{\ln \left(V_{r t}^{2}+1\right)}$ & 0,134 & 0,131 \\
\hline$Q_{\delta}=\sqrt{\ln \left(V_{\delta}^{2}+1\right)}$ & 0,134 & 0,163 \\
\hline$Q=\sqrt{\ln \left(V_{\delta}^{2}+V_{r t}^{2}+1\right)}$ & 0,189 & 0,208 \\
\hline$N_{g r, k}=b \cdot g_{r t}\left(\underline{X}_{m}\right) \cdot \exp \left(-k_{\infty} \frac{Q_{r t}^{2}}{Q}-k_{n} \frac{Q_{\delta}^{2}}{Q}-0,5 Q^{2}\right)$ & $0,819 \cdot g_{r t}\left(\underline{X}_{m}\right)$ & $0,775 \cdot g_{r t}\left(\underline{X}_{m}\right)$ \\
\hline \multicolumn{3}{|l|}{ Bemessungswert $r_{d}$ der Widerstandsfunktion } \\
\hline$N_{g r, d}=b \cdot g_{r t}\left(\underline{X}_{m}\right) \cdot \exp \left(-k_{d, \infty} \frac{Q_{r t}^{2}}{Q}-k_{d, n} \frac{Q_{\delta}^{2}}{Q}-0,5 Q^{2}\right)$ & $0,622 \cdot g_{r t}\left(\underline{X}_{m}\right)$ & $0,565 \cdot g_{r t}\left(\underline{X}_{m}\right)$ \\
\hline \multicolumn{3}{|l|}{ Teilsicherheitsbeiwert des Widerstandes } \\
\hline$\gamma_{M}=\frac{N_{g r, k}}{N_{g r, d}}$ & 1,317 & 1,372 \\
\hline$k_{c}=\frac{g_{r t}\left(\underline{X}_{k}\right)}{g_{r t}\left(\underline{X}_{m}\right)}$ & $0,74 \ldots 0,78$ & $0,74 \ldots 0,88$ \\
\hline$\gamma_{M}^{*}=k_{c, \max } \cdot \gamma_{M}$ & 1,024 & 1,207 \\
\hline
\end{tabular}




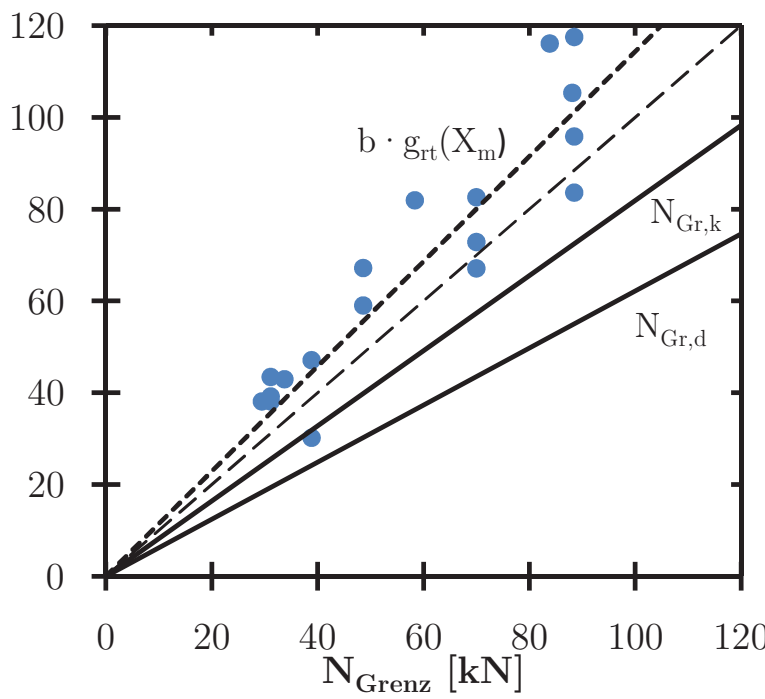

Winkelprofilanschlüsse mit einem Verbindungsmittel

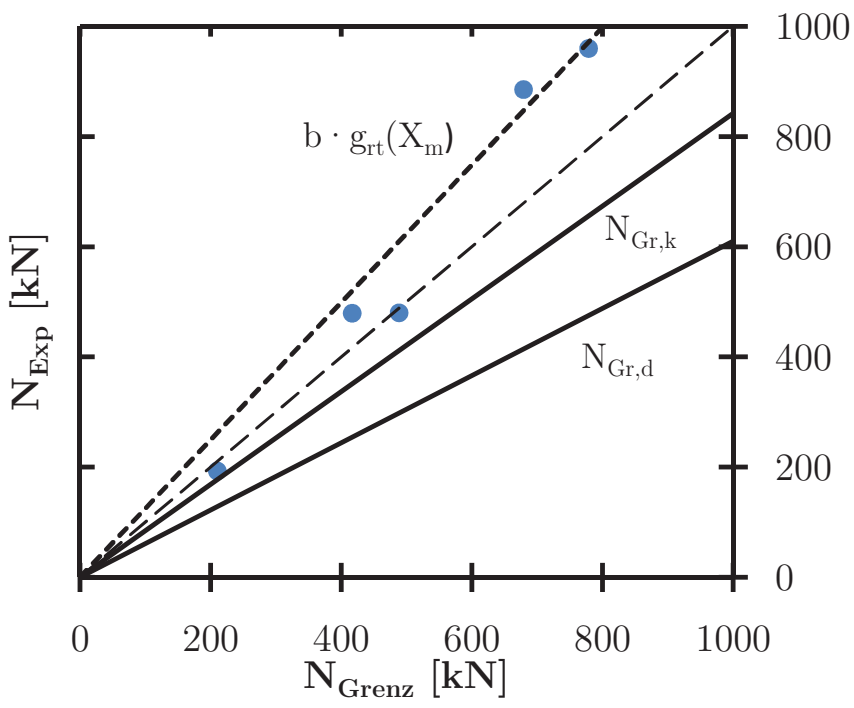

Winkelprofilanschlüsse mit mehreren Verbindungsmitteln

Abb. 5.5.: Statistische Auswertung der experimentell und rechnerisch ermittelten Traglasten

Nennwerte im Sinne der Norm sind Profilabmessungen und Sollgrößen von Loch- und Randabständen sowie charakteristische Werte von Werkstoffkennwerten. Der charakteristische Wert der Bruchzähigkeit $K_{J c}$ von Flussstahl, in Abhängigkeit der Bauteiltemperatur $T$, wird gemäß der Auswertungen nach dem Master-Curve-Konzept mit dem Wert $K_{J c-1 T, 5 \%}$, entsprechend Gleichung 3.25, festgelegt. Die Bestimmung des charakteristischen Wertes der Streckgrenze $R_{e L}$ erfolgt, aufbauend auf den Untersuchungen von LANGENBERG (1996) und STÖTZEL (1998), in Abhängigkeit der maßgebenden Bauteiltemperatur $T$ nach folgender Gleichung:

$$
R_{e L, 5 \%}=248-0,68 \cdot T \quad\left[\mathrm{~N} / \mathrm{mm}^{2}\right]
$$

Unter Berücksichtigung der charakteristischen Werkstoffkennwerte ergeben sich, anhand der ausgewerteten Versuchsdaten für den Anschluss mit einem Verbindungsmittel, Korrekturfaktoren $k_{c}$ von 0,74 bis 0,78 und für die Anschlüsse mit mehreren Verbindungsmitteln zwischen 0,74 und 0,88. Daraus resultieren auf der sicheren Seite die korrigierten Teilsicherheitsbeiwerte $\gamma_{M}{ }^{*}$ zu 1,02 bzw. 1,21 (siehe Tabelle 5.3).

\subsection{Vergleich der rechnerischen Grenzlast mit normativen Traglasten}

Um die mit den Berechnungsmodellen ermittelten Grenzlasten zur Vermeidung spröden Bauteilversagens bewerten zu können, werden sie für die geprüften Anschlüsse aus einem Verbindungsmittel mit den rechnerischen Traglasten verschiedener Bemessungsnormen gegenübergestellt. Zum einen erfolgt der Vergleich mit der Traglast nach einer der ersten Bemessungsnormen für derartige Anschlüsse, der Vorschrift für den Bau von Starkstrom-Freileitungen 
(V.S.F.) [VDEH (1930)]. Diese Norm legte fest, dass „nur der Querschnitt des genieteten Schenkels, nach Abzug der Schwächung durch Bohrung, in Rechnung zu setzen" ist. Die zulässige Zugbeanspruchung für einen Flussstahl Normalgüte St 37.12 entsprach 160 N/mm².

Des Weiteren werden die berechneten Grenzlasten den Bemessungswerten der Traglast nach DIN EN 1993-1-8:2010 gegenübergestellt. Für Anschlüsse mit einem Verbindungsmittel ist die Traglast in Abschnitt 3.10.3 nach folgender Gleichung festgelegt:

$$
N_{u, R d}=\frac{\left(2 \cdot e_{2}-d_{0}\right) \cdot t \cdot f_{u}}{\gamma_{M 2}} \text { mit: } \gamma_{M 2}=1,25
$$

Dabei wird vorausgesetzt, dass ein Versagen durch Überschreitung der Lochleibungsbeanspruchbarkeit nicht stattfindet.

Für den hier angestellten Vergleich wird die Zugfestigkeit von Flussstahl, gemäß der Angaben von Cremona; PAtron et al. (2007), mit $335 \mathrm{~N} / \mathrm{mm}^{2}$ in Rechnung gestellt. Um die Grenzlasten auf charakteristischem Lastniveau vergleichen zu können, wird $N_{u, R d}$ zusätzlich durch den Teilsicherheitsbeiwert der Beanspruchung $\gamma_{F}$ dividiert, der näherungsweise mit 1,45 angenommen wird. Die Ergebnisse dieser Vergleichsrechnungen sind in Abbildung 5.6 dargestellt.

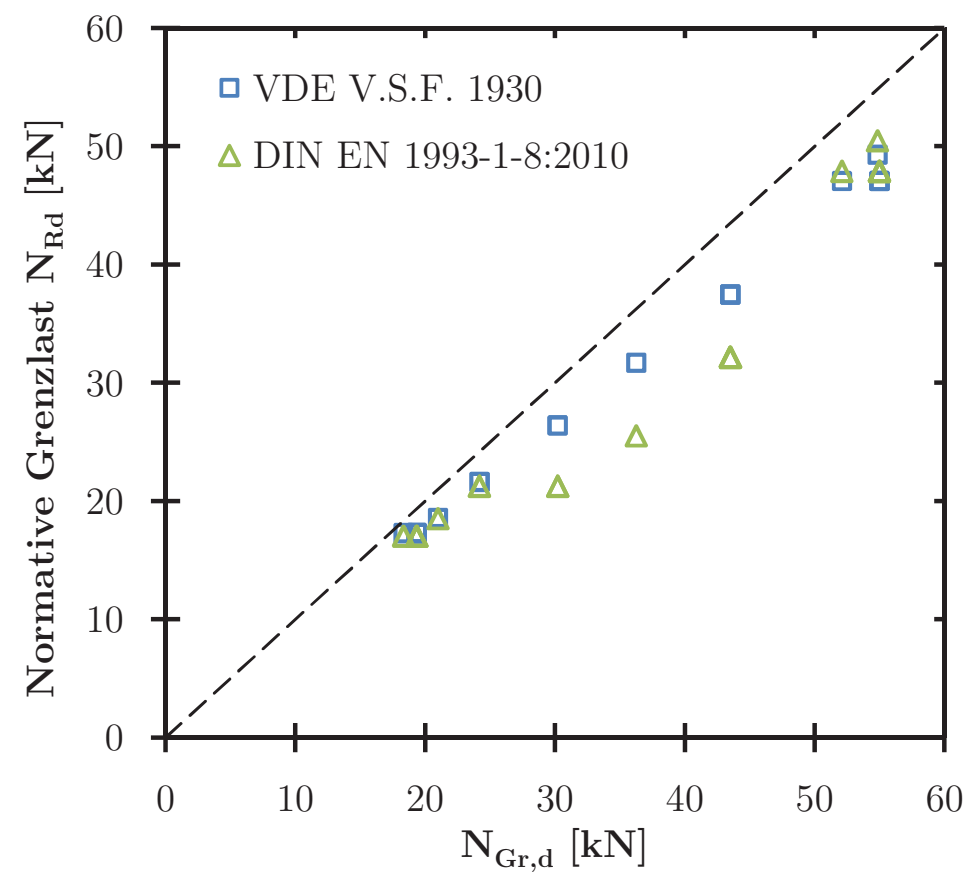

Abb. 5.6.: Vergleich der ermittelten Grenzlasten zur Vermeidung von Sprödbruch mit den Traglasten geeigneter Bemessungsnormen für die in Zugversuchen geprüften Winkelprofilanschlüsse mit einem Verbindungsmittel

Es wird deutlich, dass die rechnerischen Traglasten, für die in Zugversuchen geprüften Bauteile mit einem Verbindungsmittel, nach den angegebenen Normen kleiner sind, als die mit dem in Abschnitt 5.3.1 hergeleiteten Bemessungsmodell ermittelten Grenzlasten. Unter Einhaltung der normativen Traglasten ist bei der im Versuch realisierten Bauteiltemperatur für diese Bauteile kein Nachweis gegen Sprödbruch erforderlich. 


\subsection{Zusammenfassung der Bemessungsmodelle}

Auf Basis der durchgeführten Bauteilzugversuche, den Erkenntnissen aus den numerischen Berechnungen sowie theoretischen Überlegungen und statistischen Auswertungen wurden Nachweiskonzepte von Winkelprofilanschlüssen, mit einem und mehreren Verbindungsmitteln, zur Vermeidung von Sprödbruch entwickelt. Die Bemessungsansätze sind nur im Bereich der numerisch und experimentell überprüften Bauteil- und Anschlussgeometrien gültig. Die zugrunde gelegten Materialeigenschaften entsprechen den Mindestwerten allgemeiner Flussstähle im Rahmen der in Abschnitt 3.4.3 experimentell untersuchten Werkstoffchargen. Liegen für die zu untersuchenden Bauteile gesicherte Erkenntnisse über die tatsächlichen Eigenschaften vor, können diese entsprechend der Erläuterungen in Abschnitt 5.3 verwendet werden. Die in Abschnitt 5.4 ermittelten Teilsicherheitsbeiwerte $\gamma_{M}{ }^{*}$ der Widerstandsfunktionen wurden in Sicherheitsbeiwerte der Werkstoffwiderstände $R_{e L}$ und $K_{\text {mat }}$ überführt. Die Bemessungsmodelle umfassen nur den Nachweis der gelochten Bauteile. Die Tragfähigkeit der Verbindungsmittel ist gesondert zu betrachten. Eine Übersicht der Nachweiskonzepte mit der jeweils zugehörigen Anschlussgeometrie und allen Beiwerten ist in den Tabellen 5.4 und 5.5 dargestellt. 
Tab. 5.4.: Zusammenfassung des Bemessungsmodells für Winkelprofilanschlüsse mit einem Verbindungsmittel

\begin{tabular}{|c|c|}
\hline \multicolumn{2}{|r|}{ Grenzlast für Anschlüsse mit einem Verbindungsmittel } \\
\hline & $\begin{array}{l}a=3 \mathrm{~mm} \\
40 \mathrm{~mm} \leq b \leq 90 \mathrm{~mm} \\
0,25 \leq 2 a_{e q} / b \leq 0,5 \\
0,25 \leq h / b \leq 0,5 \\
h_{e f f}=h-\frac{d}{3} \cdot \frac{(h+d) / 2}{2 a_{e q}} \\
W_{e f f}=2 c\end{array}$ \\
\hline Grenzlast & $\begin{array}{l}N_{\text {grenz }}=\frac{1}{\gamma_{M^{*}}} \cdot \sqrt{\sqrt{F_{Y}^{4}+\left(\frac{\sqrt{8} \cdot F_{Y} \cdot K_{\text {mat }} \cdot W_{\text {eff }} \cdot t}{\sqrt{\pi a} \cdot \beta_{F}}\right)^{2}}-F_{Y}^{2} \leq \frac{F_{Y}}{\gamma_{M^{*}}}} \\
\text { mit } F_{Y}=\left(W-2 a_{e q}\right) \cdot t \cdot f_{y} \quad \text { und } \gamma_{M}^{*}=1,02\end{array}$ \\
\hline $\begin{array}{l}\text { Werkstoff- } \\
\text { kennwerte }\end{array}$ & $\begin{array}{l}f_{y}=R_{e L}(T)=248-0,68 \cdot T \quad\left[\mathrm{~N} / \mathrm{mm}^{2}\right] \\
K_{\text {mat }}=K_{J c}(T)=25,9+29,7 \cdot \exp [0,0186 \cdot(T+30,0)] \quad[\mathrm{MPa} \sqrt{\mathrm{m}}]\end{array}$ \\
\hline Geometrie & $\begin{array}{l}\beta_{F}=\left[\frac{W_{e f f}}{\sqrt{2 \pi a c}} \cdot F_{P} \cdot F_{H P W}+\left(F_{F W}+2 \cdot F_{E C C}\right) \cdot F_{T H}\right] \cdot F_{L} \\
F_{P}=\left\{1+0,2967 \sqrt{1-\left(g / a_{e q}\right)^{2}}\left[1-\cos \left(\frac{\pi a_{e q}}{2 c}\right)\right]\right\} \cdot F_{I I I} \\
F_{I I I}=\frac{\sqrt{\tan \left(\frac{\pi a_{e q}}{2 c}\right)}\left\{1-\left[\sin \left(\frac{\pi g}{2 c}\right) / \sin \left(\frac{\pi a_{e q}}{2 c}\right)\right]\right\}}{\sqrt{1-\left[\cos \left(\frac{\pi a_{e q}}{2 c}\right) / \cos \left(\frac{\pi g}{2 c}\right)\right]^{2}}} \\
F_{H P W}=\left[23,76\left(\frac{h_{e f f}}{W}\right)^{2}-28,558\left(\frac{h_{e f f}}{W}\right)+8,913\right]\left(\frac{2 a_{e q}}{W}\right)+1,284\left(\frac{h_{e f f}}{W}\right)+0,322 \\
F_{F W}=\sqrt{\sec \left[\frac{\pi a_{e q}}{2 c}\right]} \quad F_{E C C}=-0,6173\left(\frac{a_{e q}}{c}\right)^{2,6} \cdot\left(\frac{2 e}{W}\right)^{2} \\
F_{T H}=e^{\left[1,2133-2,205(a /(a+R))+0,645(a /(a+R))^{2}\right]} \\
F_{L}=1,113-0,00252 \cdot \frac{W^{2}}{c \cdot h} \cdot \frac{a_{e q}}{a_{0}} \quad \operatorname{mit}: a_{0}=1 \mathrm{~mm}\end{array}$ \\
\hline
\end{tabular}


Tab. 5.5.: Zusammenfassung des Bemessungsmodells für zentrische Winkelprofilanschlüsse mit mehreren Verbindungsmitteln

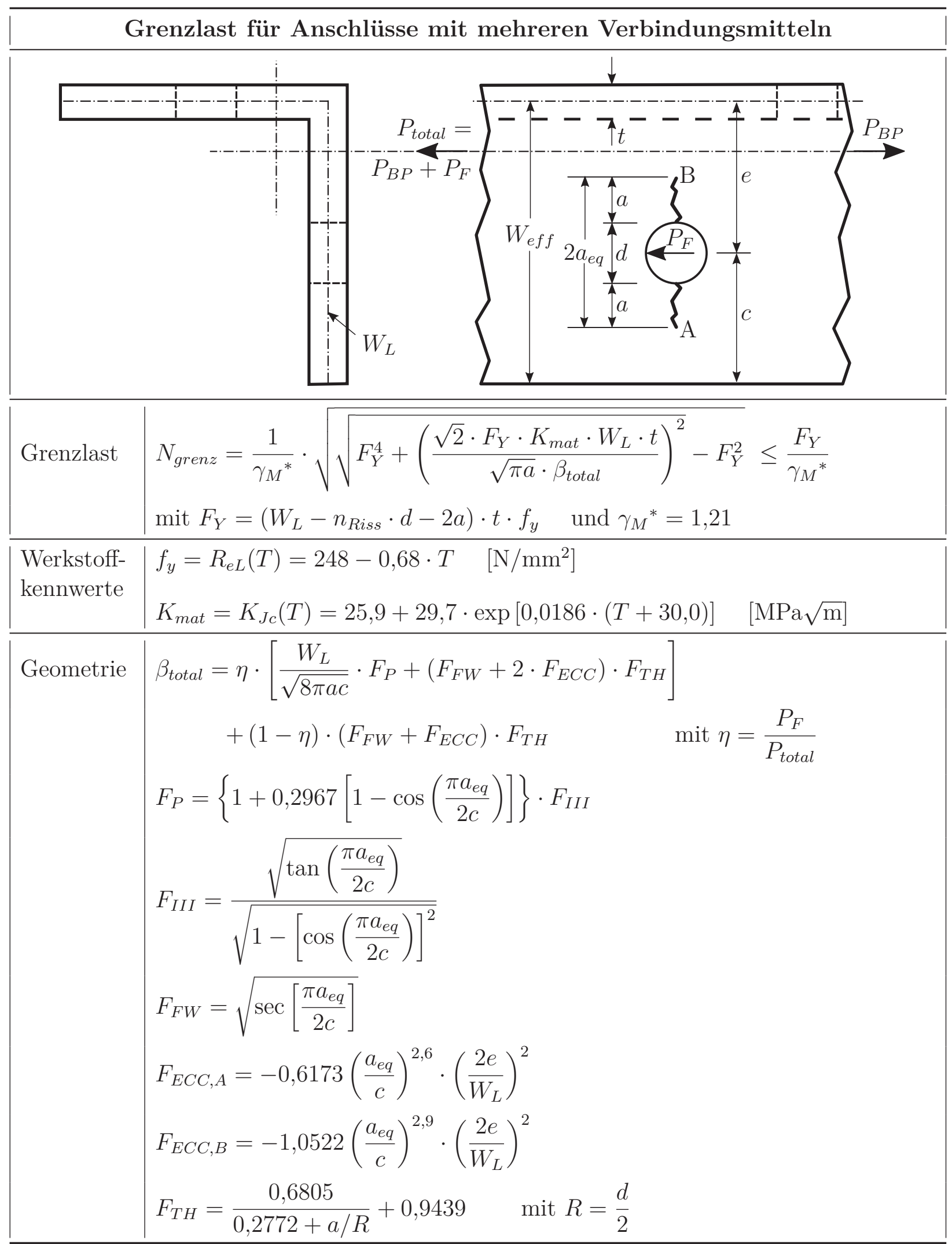




\section{Kapitel 6.}

\section{Anwendungsbeispiele}

\subsection{Allgemeines}

Die Anwendung der in Kapitel 3 ermittelten Bruchzähigkeiten alter Flussstähle, wie auch das in Kapitel 5 abgeleitete Verfahren zur bruchmechanischen Sicherheitsanalyse gelochter Bauteile des Stahlhochbaus, werden nachfolgend an zwei Beispielen aus der Praxis demonstriert. Die Beispiele konzentrieren sich dabei auf ausgewählte, hoch zugbeanspruchte Konstruktionsdetails. Sie beschreiben die Anwendung statistisch abgesicherter Mindestwerte der Bruchzähigkeit, ebenso wie eine Möglichkeit zum Ansatz höherer Zähigkeitskennwerte durch Analyse von Materialproben und Nachweis besserer Werkstoffgüte.

\subsection{Beispiel 1: Anschlüsse in einem Stahlgittermast}

Um den Einsatz der in Kapitel 5 abgeleiteten Bemessungsmodelle für Winkelprofilanschlüsse $\mathrm{zu}$ veranschaulichen, werden im ersten Anwendungsbeispiel verschiedene Nietverbindungen in einem Freileitungsmast untersucht. Wie bereits in Abschnitt 2.3 erläutert, stellen die Stahlgittermaste im Freileitungsnetz Deutschlands einen in Größe und Anzahl wesentlichen Bestandteil alter Stahlkonstruktionen dar. Ihre meist sehr schlanken Konstruktionen bestehen fast ausschließlich aus Winkelprofilen. Die Anschlüsse der Diagonalen an den Eckstielen wurden sehr häufig, um aufwendige Knotenbleche zu vermeiden, mit nur einem Niet größeren Durchmessers ausgeführt (siehe auch Tabelle 2.6). Eine in den 1930er Jahren typische Konstruktionsform war der Portalmast. Er bestand aus zwei schlanken Fachwerkschäften und einer gemeinsamen, die Leitungen tragenden Traverse am Kopf. Die Geometrie eines solchen Mastes sowie wesentliche Anschlussdetails sind in Abbildung 6.1 dargestellt.

Der Anschluss der Diagonalenwinkel L60x6 erfolgt an einem Schenkel mit jeweils einem Niet mit 17 mm Durchmesser. Unter Ansatz der Abmessungen des Winkelprofils, der realisierten Randabstände des Anschlussniets und der Annahme eines einseitigen Anrisses am Lochrand mit $a=3 \mathrm{~mm}$, ergibt sich nach Tabelle 5.4 ein bezogener Spannungsintensitätsfaktor von:

$$
\beta_{F}=\left[\frac{47}{\sqrt{2 \pi \cdot 3 \cdot 23,5}} \cdot 1,289 \cdot 1,288+1,123 \cdot 1,978\right] \cdot 0,984=5,833
$$

Da für die zahlreichen, potentiell sprödbruchgefährdeten Bauteile eines Stahlgittermastes die tatsächlichen Werkstoffkennwerte nicht bekannt sind, erfolgt die Annahme dieser Werte nach Tabelle 5.4. Bei einer maßgebenden Einsatztemperatur des Mastes von $-30^{\circ} \mathrm{C}$ ergibt sich 


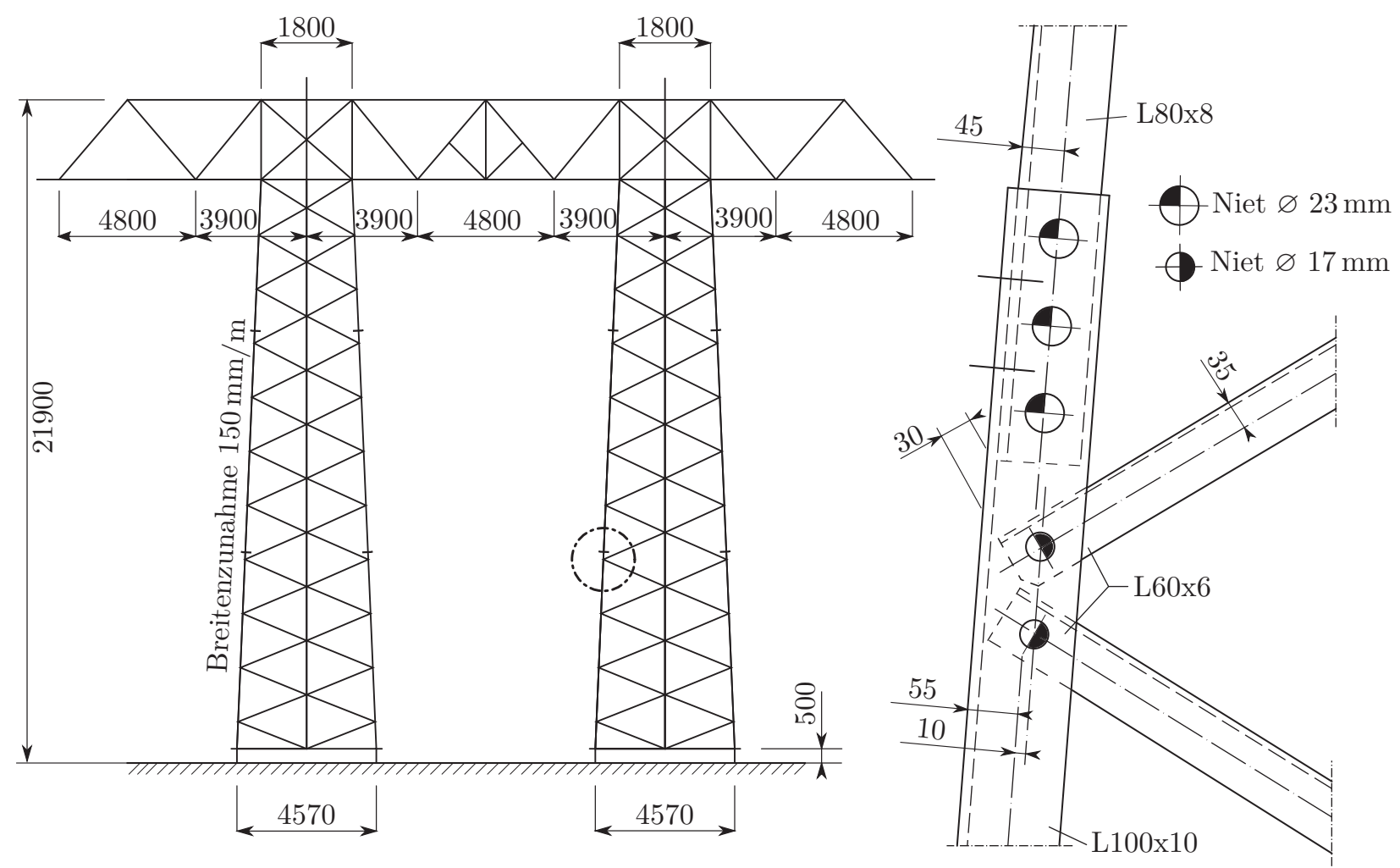

Abb. 6.1.: Geometrie des Portalmastes und Anschlussdetails des Eckstiels und der Diagonalen

die Streckgrenze zu $R_{e L}=263,1 \mathrm{~N} / \mathrm{mm}^{2}$ und die Bruchzähigkeit zu $K_{m a t}=54,5 \mathrm{MPa} \sqrt{\mathrm{m}}$. Damit errechnet sich die Grenzlast im Anschluss der Diagonale (ohne einen Nachweis auf Lochleibung) zu $N_{\text {grenz }}=63,2 \mathrm{kN}$.

Die Eckstielquerschnitte des Mastes sind über die Höhe entsprechend der Beanspruchung abgestuft. Der Eckstielstoß erfolgt ebenfalls ohne zusätzliche Knotenbleche durch Anfasen und Einstellen des kleineren Winkelprofils in das Größere. Zur Kraftübertragung dienen fünf Niete mit $23 \mathrm{~mm}$ Durchmesser, verteilt auf beide Winkelschenkel. Maßgebend für den Nachweis des Anschlusses ist der kleinere Winkel L80x8. Unter der Annahme eines beidseitig symmetrischen Anrisses am Rand des obersten Nietloches mit $a=3 \mathrm{~mm}$, ergibt sich nach Tabelle 5.5 ein bezogener Spannungsintensitätsfaktor von:

$$
\beta_{\text {total }}=0,2 \cdot\left[\frac{152}{\sqrt{8 \pi \cdot 3 \cdot 35}} \cdot 1,528+1,085 \cdot 2,209\right]+(1-0,2) \cdot 1,103 \cdot 2,209=3,332
$$

Die Streckgrenze des Materials ergibt sich nach Tabelle 5.5 für die Temperatur von $-30^{\circ} \mathrm{C}$ zu $R_{e L}=221,8 \mathrm{~N} / \mathrm{mm}^{2}$ und die Bruchzähigkeit zu $K_{m a t}=46,0 \mathrm{MPa} \sqrt{\mathrm{m}}$. Damit errechnet sich die Grenzlast des Eckstielstoßes zu $N_{\text {grenz }}=218,3 \mathrm{kN}$.

Der Nachweis des unteren Eckstielprofils L100x10 im Bereich des Anschlusses der Diagonalen kann vereinfachend ebenfalls nach Tabelle 5.5 erfolgen. Die am Nietloch eingeleitete Kraft entspricht der Vertikalkomponente der Diagonalenkraft. Diese ist klein im Vergleich zur Zugkraft im Eckstiel. Der Faktor $\eta$ geht damit gegen Null und die bezogene Spannungsintensität $\beta_{\text {total }}$ ist auch bei Berücksichtigung der exzentrischen Lasteinleitung deutlich kleiner als im Stoßbereich. Dieser Nachweis wird folglich nicht maßgebend. 


\subsection{Beispiel 2: Geschweißter Anschluss des Zuggurtes eines Fachwerkträgers}

Die Gefahr eines Sprödbruches in Stahltragwerken wird neben werkstoffspezifischen und einsatzbedingten vor allem auch durch konstruktionsbedingte Einflüsse bestimmt. So sind geschweißte Konstruktionsdetails, aufgrund ihrer größeren Kerbwirkung und den eingeprägten Eigenspannungen, häufig stärker sprödbruchgefährdet als genietete oder geschraubte Details. Darüber hinaus wirkt eine hohe Zugbeanspruchung in Bereichen der Konstruktion mit stärkerer Kerbwirkung als sprödbruchfördernd.

Gegenstand dieses Anwendungsbeispiels ist deshalb ein Dreigurt-Fachwerkbinder aus den 1930er Jahren in gemischter Bauweise. Verschiedene Bauteile der Konstruktion, so auch der Zuggurt, wurden im Werk in transportablen Segmenten geschweißt und anschließend auf der Baustelle über Nietverbindungen zum Gesamttragwerk zusammengebaut.

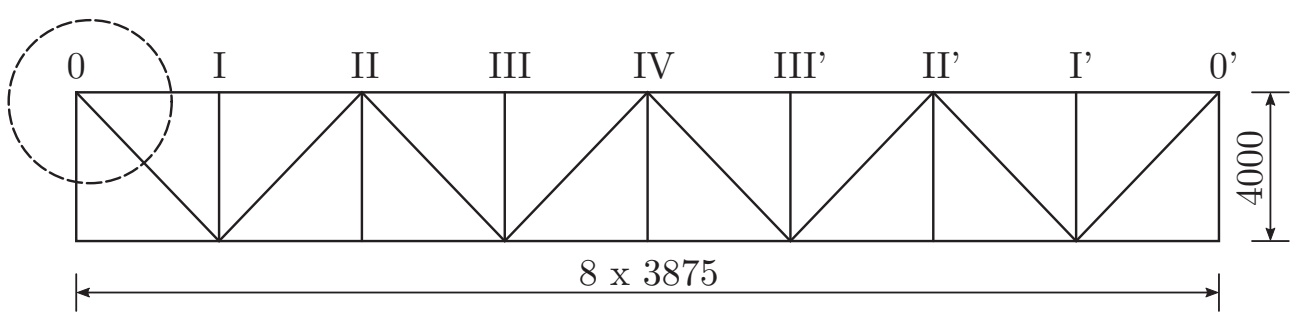

Schnitt A-A
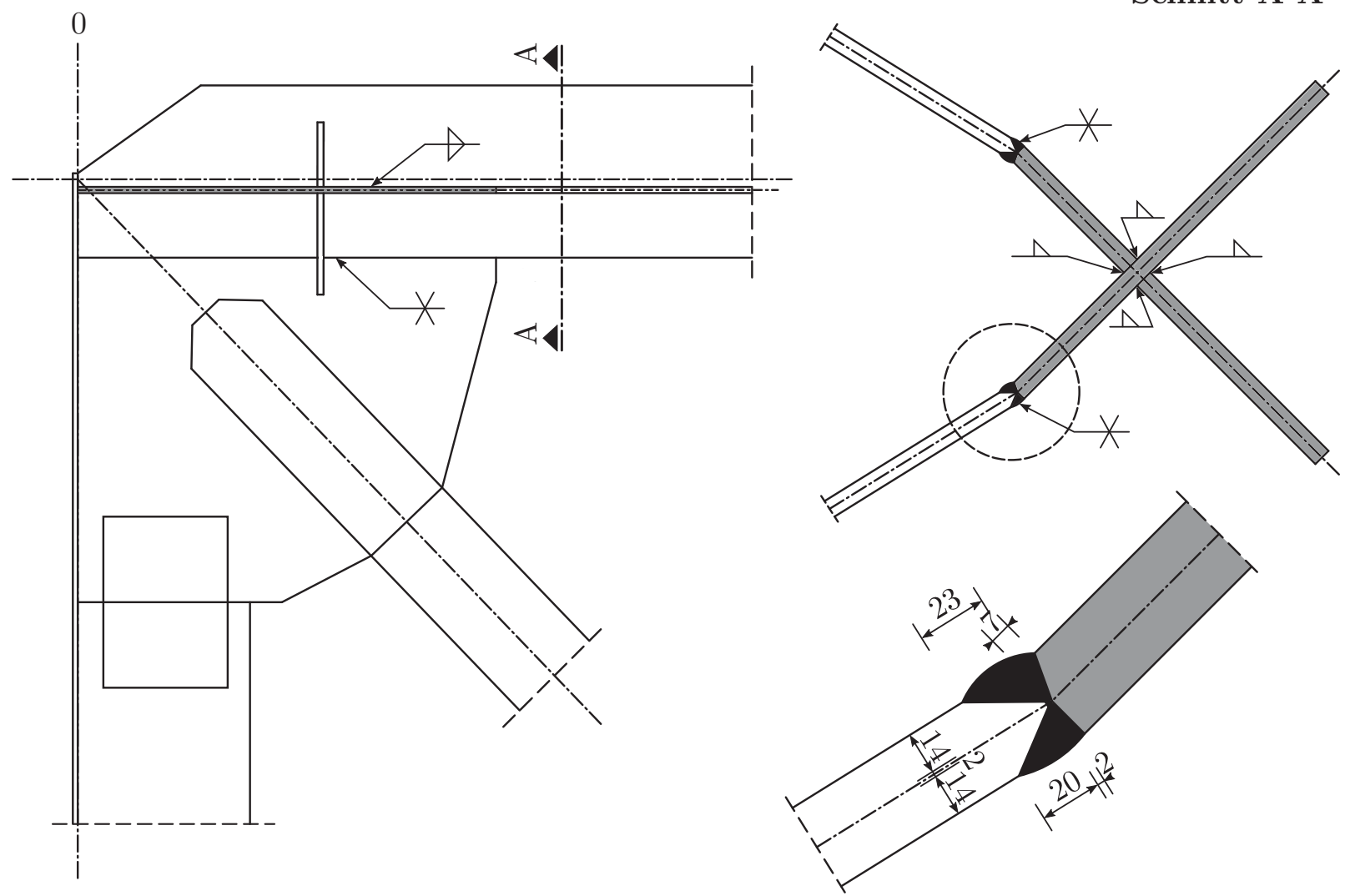

Abb. 6.2.: Geometrie und Schweißdetail des Zuggurtes und dessen Anschluss am Knotenblech in Achse 0 
Am kreuzförmigen Querschnitt des Zuggurtes schließen in den Achsen 0 und 0` die Diagonalen der beiden Fachwerk-Hauptträger über große Knotenbleche an, die wiederum mit dem Endschott des Dreigurtbinders verbunden sind. Die Knotenbleche und die Stegbleche des Gurtes sind mit einer unsymmetrischen X-Naht verschweißt (siehe Abbildung 6.2).

Die Ermittlung der maßgebenden Beanspruchung des Zuggurtes am Anschluss zum Knotenblech, in Form von Nennspannungen auf Basis von Stabschnittgrößen, ist aufgrund der komplexen konstruktiven Ausbildung und Tragwirkung nicht möglich. Die Beanspruchung wird deshalb mit Hilfe eines FE-Modells bestimmt, in dem die Knotenbleche und der Zuggurt durch Schalenelemente modelliert sind. Die Berechnung realistischer Kerbspannungen am Gurtblechrand erfolgt, nach den Empfehlungen von HoBBACHER (2008) zum Strukturspannungskonzept, durch quadratische Extrapolation aus den Spannungswerten kurz vor der geometrischen Kerbe nach Gleichung 6.1. Das Berechnungsmodell wurde gemäß der Vorgaben im Bereich des Knotenblechanschlusses entsprechend fein vernetzt. Die ermittelten Längsspannungen im Zuggurtquerschnitt sind in Abbildung 6.4 dargestellt.

$$
\sigma_{\text {hot spot }}=3 \cdot \sigma_{4 m m}-3 \cdot \sigma_{8 m m}+\sigma_{12 m m}=271 \mathrm{~N} / \mathrm{mm}^{2}
$$

Ausgehend von den maßgebenden Kerbspannungen am Rand des Zuggurtes im Übergang zum Knotenblech erfolgt an dieser Stelle die Annahme eines seitlichen Durchrisses. Die Größe des angenommenen Risses orientiert sich wie üblich an dem Ziel, diesen Riss bei einer Bauwerksprüfung finden zu können bzw. ihn gerade zu übersehen. Die Nachweisgrenzen der in der Praxis eingesetzten ZfP-Verfahren wurden von Miki; FuKAZAWA et al. (1989) systematisch untersucht. Generell sinkt die detektierbare Rissgröße mit steigendem Aufwand des Verfahrens. Unter der Annahme einer handnahen Sichtprüfung des Tragwerks wird auf Basis der Untersuchungsergebnisse in der bruchmechanischen Berechnung eine sichtbare Risslänge von $A=4 \mathrm{~mm}$ angenommen. Das für den Zuggurt zutreffende bruchmechanische Berechnungsmodell ist die Platte unter Zugbeanspruchung mit seitlichem Durchriss (siehe Abbildung 6.3) bei einer Gesamtbreite des Gurtbleches von $W=400 \mathrm{~mm}$, wobei gilt:

$$
a=\frac{A}{W}, \quad x=\frac{X}{W} \quad \text { mit } 0 \leq x \leq 1,0
$$
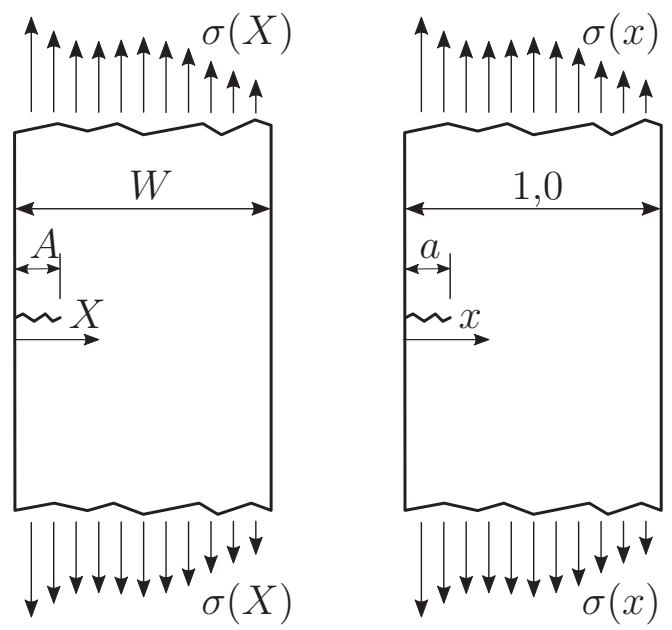

Abb. 6.3.: Geometriedefinition eines Blechstreifens mit einseitigem Randriss unter ungleichmäßiger Zugbeanspruchung nach Wu; CARLSSON (1991) 
Um den hochgradig nichtlinearen Spannungsverlauf im Zuggurt in Rissachse (siehe Abbildung 6.4) berücksichtigen zu können, erfolgt die Ermittlung des Spannungsintensitätsfaktors, mit Hilfe der Lösungen von Wu; CARLSSON (1991), mittels Gewichtsfunktionen. Hierfür wird zunächst der Spannungsverlauf in Rissachse im ungerissenen Querschnitt als Polynom 6. Grades nach folgender Form approximiert:

$$
\sigma=\sum_{n=0}^{6} \sigma_{n} \cdot x^{n} \quad \text { mit } 0 \leq x \leq 1,0
$$

Für die Ermittlung des korrekten K-Faktors ist es dabei wichtig, dass die Summe der auf die Rissflanken wirkenden Zugspannungen durch das Spannungspolynom in äquivalenter Größe abgebildet wird. Dies ist mit der gewählten Approximation ausreichend genau gegeben.

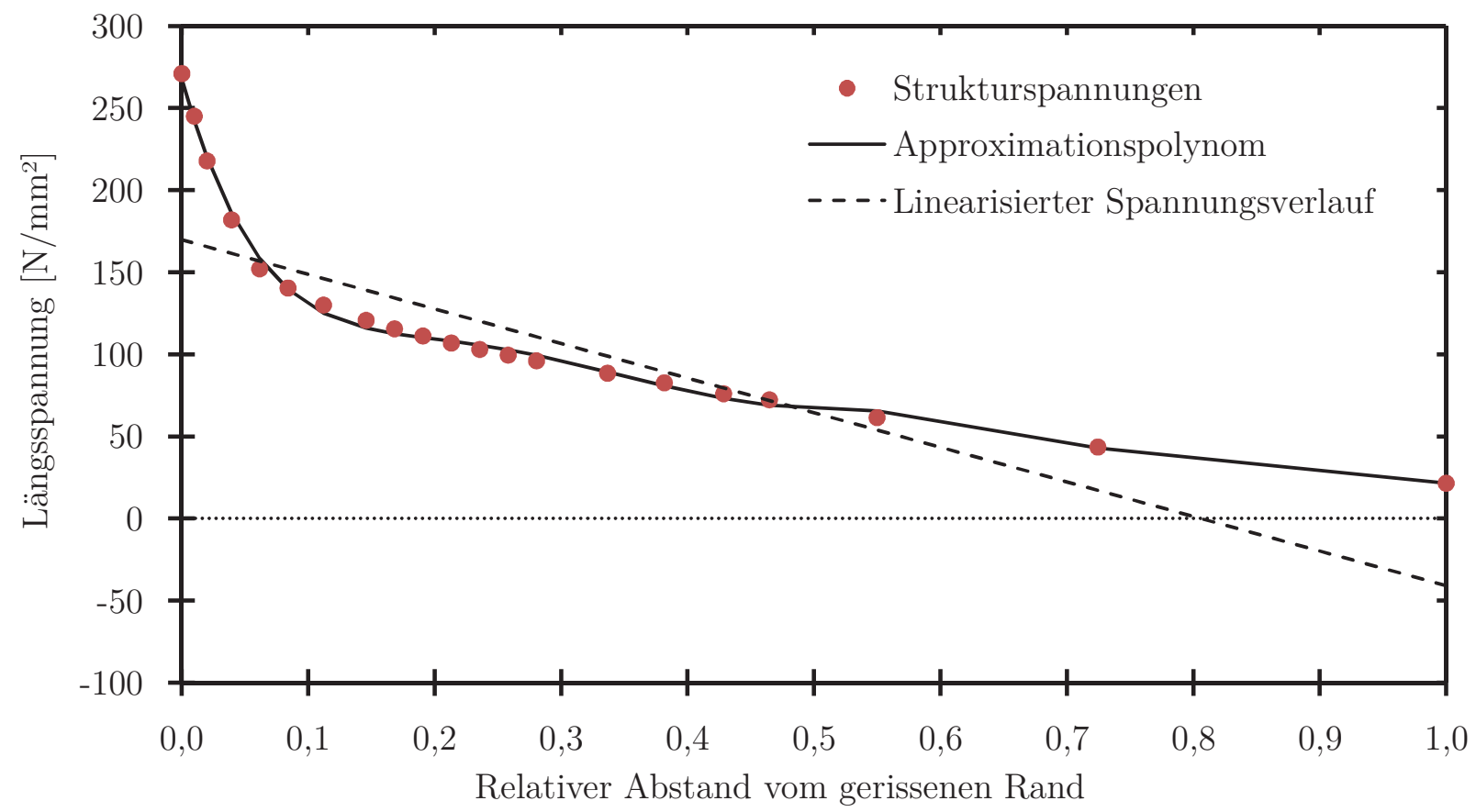

Abb. 6.4.: Approximation des Spannungsverlaufs im Zuggurt in Rissachse

Der maßgebende Spannungsintensitätsfaktor aus der äußeren Beanspruchung (Primärspannungen) ergibt sich nach Wu; CARLSSON (1991) als Summe der Intensitäten der $n+1$ Spannungskomponenten zu:

$$
K_{I}^{p}=\left(\sum_{n=0}^{6} f_{n}(a) \cdot \sigma_{n}\right) \cdot \sqrt{\pi \cdot a \cdot W}=31,7 \mathrm{MPa} \sqrt{\mathrm{m}}
$$

Die Werte $\sigma_{n}$ entsprechen den Koeffizienten des Spannungspolynoms nach Gleichung 6.3 und die Faktoren $f_{n}(a)$ den in Tabelle 6.1 angegebenen, normierten Spannungsintensitäten.

Zur Ermittlung der plastischen Grenzlast der einseitig angerissenen Platte unter Zugbeanspruchung existiert eine geeignete Lösung in der FKM-Richtlinie [BERGER; BLAUEL et al. (2006)]. Die Größe der Ligamentplastizierung $L_{r}$ in Abhängigkeit der bezogenen Risslänge $a$ und der Streckgrenze $R_{e L, 5 \%}(T)$ nach Gleichung 5.12 ergibt sich zu: 
Tab. 6.1.: Spannungsintensitätsfaktor $f_{n}$ für einen Randriss in einem Blechstreifen unter einer Zugspannung mit dem Potenzansatz $\sigma(x) / \sigma=x^{n}$ nach Wu; CARLsson (1991)

\begin{tabular}{c|rcrcccc}
\hline & \multicolumn{7}{c}{$n$} \\
\hline$a$ & 0 & 1 & 2 & 3 & 4 & 5 & 6 \\
\hline 0,01 & 1,1226 & $6,824 \mathrm{E}-03$ & $5,246 \mathrm{E}-05$ & $4,401 \mathrm{E}-07$ & $3,860 \mathrm{E}-09$ & $3,477 \mathrm{E}-11$ & $3,189 \mathrm{E}-13$ \\
0,05 & 1,1402 & $3,447 \mathrm{E}-02$ & $1,322 \mathrm{E}-03$ & $5,535 \mathrm{E}-05$ & $2,424 \mathrm{E}-06$ & $1,091 \mathrm{E}-07$ & $5,001 \mathrm{E}-09$ \\
0,10 & 1,1890 & $7,089 \mathrm{E}-02$ & $5,398 \mathrm{E}-03$ & $4,502 \mathrm{E}-04$ & $3,933 \mathrm{E}-05$ & $3,534 \mathrm{E}-06$ & $3,234 \mathrm{E}-07$ \\
0,20 & 1,3672 & $1,558 \mathrm{E}-01$ & $2,317 \mathrm{E}-02$ & $3,811 \mathrm{E}-03$ & $6,597 \mathrm{E}-04$ & $1,177 \mathrm{E}-04$ & $2,144 \mathrm{E}-05$ \\
0,30 & 1,6602 & $2,675 \mathrm{E}-01$ & $5,783 \mathrm{E}-02$ & $1,398 \mathrm{E}-02$ & $3,582 \mathrm{E}-03$ & $9,496 \mathrm{E}-04$ & $2,575 \mathrm{E}-04$ \\
0,40 & 2,1113 & $4,249 \mathrm{E}-01$ & $1,179 \mathrm{E}-01$ & $3,708 \mathrm{E}-02$ & $1,245 \mathrm{E}-02$ & $4,344 \mathrm{E}-03$ & $1,555 \mathrm{E}-03$ \\
0,50 & 2,8241 & $6,630 \mathrm{E}-01$ & $2,201 \mathrm{E}-01$ & $8,401 \mathrm{E}-02$ & $3,450 \mathrm{E}-02$ & $1,481 \mathrm{E}-02$ & $6,545 \mathrm{E}-03$ \\
0,60 & 4,0333 & $1,058 \mathrm{E}+00$ & $4,014 \mathrm{E}-01$ & $1,775 \mathrm{E}-01$ & $8,518 \mathrm{E}-02$ & $4,299 \mathrm{E}-02$ & $2,243 \mathrm{E}-02$ \\
0,70 & 6,3558 & $1,810 \mathrm{E}+00$ & $7,586 \mathrm{E}-01$ & $3,752 \mathrm{E}-01$ & $2,033 \mathrm{E}-01$ & $1,166 \mathrm{E}-01$ & $6,944 \mathrm{E}-02$ \\
0,75 & 8,4537 & $2,487 \mathrm{E}+00$ & $1,085 \mathrm{E}+00$ & $5,613 \mathrm{E}-01$ & $3,195 \mathrm{E}-01$ & $1,931 \mathrm{E}-01$ & $1,216 \mathrm{E}-01$ \\
0,80 & 11,9548 & $3,618 \mathrm{E}+00$ & $1,631 \mathrm{E}+00$ & $8,773 \mathrm{E}-01$ & $5,210 \mathrm{E}-01$ & $3,296 \mathrm{E}-01$ & $2,177 \mathrm{E}-01$ \\
0,85 & 18,6264 & $5,776 \mathrm{E}+00$ & $2,679 \mathrm{E}+00$ & $1,487 \mathrm{E}+00$ & $9,147 \mathrm{E}-01$ & $6,012 \mathrm{E}-01$ & $4,138 \mathrm{E}-01$ \\
0,90 & 34,6348 & $1,097 \mathrm{E}+01$ & $5,203 \mathrm{E}+00$ & $2,962 \mathrm{E}+00$ & $1,873 \mathrm{E}+00$ & $1,269 \mathrm{E}+00$ & $9,025 \mathrm{E}-01$ \\
\hline
\end{tabular}

$$
L_{r}=\frac{a \cdot \sigma_{m}+\frac{\sigma_{b}}{3}+\sqrt{\left(a \cdot \sigma_{m}+\frac{\sigma_{b}}{3}\right)^{2}+(1-a)^{2} \cdot \sigma_{m}^{2}}}{(1-a)^{2} \cdot R_{e}}=0,41
$$

Da dieser Ansatz nur für eine linear veränderliche Zugbeanspruchung gilt, wird die Zugspannung im Querschnitt in eine äquivalente Spannungsverteilung umgerechnet. Diese setzt sich aus einer konstanten Spannung $\sigma_{m}$ und einem Biegeanteil $\sigma_{b}$ zusammen. Diese Umrechnung gelingt mit Hilfe der folgenden Gleichungen auf Basis der Koeffizienten des oben beschriebenen Spannungspolynoms. Dieser linearisierte Spannungsverlauf ist in Abbildung 6.4 ebenfalls dargestellt.

$$
\begin{aligned}
& \sigma_{m}=\sigma_{0}+\frac{1}{2} \sigma_{1}+\frac{1}{3} \sigma_{2}+\frac{1}{4} \sigma_{3}+\frac{1}{5} \sigma_{4}+\frac{1}{6} \sigma_{5}+\frac{1}{7} \sigma_{6}=64,5 \mathrm{~N} / \mathrm{mm}^{2} \\
& \sigma_{b}=-\frac{1}{2} \sigma_{1}-\frac{1}{2} \sigma_{2}-\frac{9}{20} \sigma_{3}-\frac{2}{5} \sigma_{4}-\frac{5}{14} \sigma_{5}-\frac{9}{28} \sigma_{6}=105,2 \mathrm{~N} / \mathrm{mm}^{2}
\end{aligned}
$$

Neben der primären Beanspruchung aus äußeren Lasten müssen bei diesem geschweißten Konstruktionsdetail sekundäre Beanspruchungen, infolge der Schweißeigenspannungen, berücksichtigt werden. Angaben über Größe und Verteilung dieser Eigenspannungen sind bei BARThelemy (1999) zu finden. Für die im Anschluss des Knotenblechs ausgeführte XNaht sind für die Überlagerung mit den Zugspannungen im Zuggurt die Eigenspannungen in Nahtlängsrichtung entscheidend. Diese müssen im Bereich der Schweißnaht konservativ in Höhe der Streckgrenze $R_{e}$ des Grundmaterials angesetzt werden und klingen dann über die Breite $y_{0}$ bis auf null ab (siehe Abbildung 6.5). Diese Breite der Fließzone ergibt sich in Abhängigkeit der Blechdicke $t$ und verschiedener Schweißparameter wie folgt:

$$
y_{0}=\frac{1,033 \cdot K}{R_{e}} \cdot \frac{\eta \cdot q_{s} \cdot A_{w}}{t}
$$


wenn: $\quad t<r_{0}=\sqrt{\frac{K \cdot \eta \cdot q_{s} \cdot A_{w}}{R_{e}}}$

mit: $\quad K=153 \mathrm{Nmm} / \mathrm{J} \quad$ (Werkstoffkonstante für ferritischen Stahl)

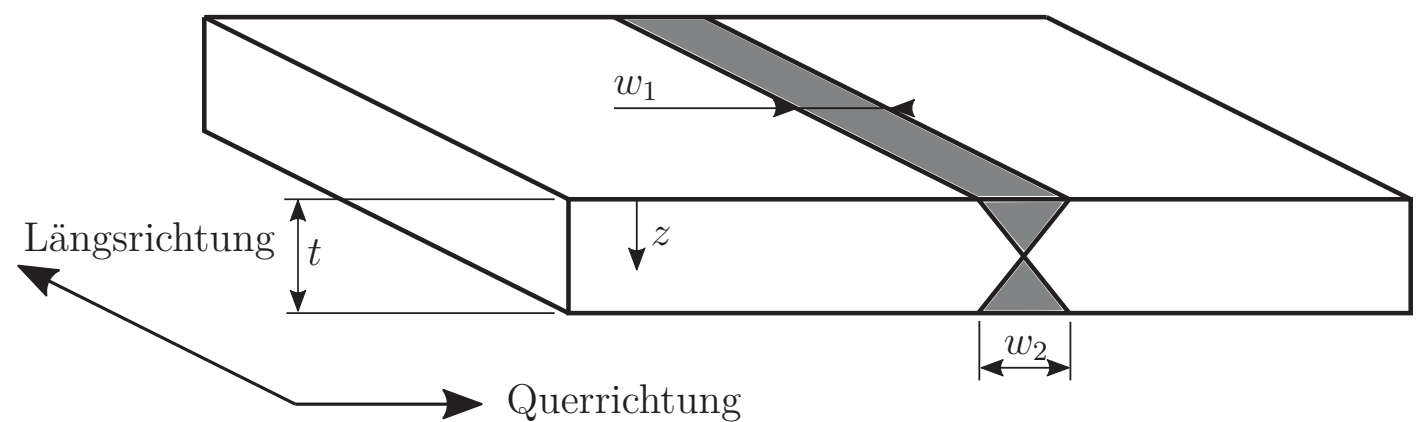

Querrichtung

Nahtgeometrie
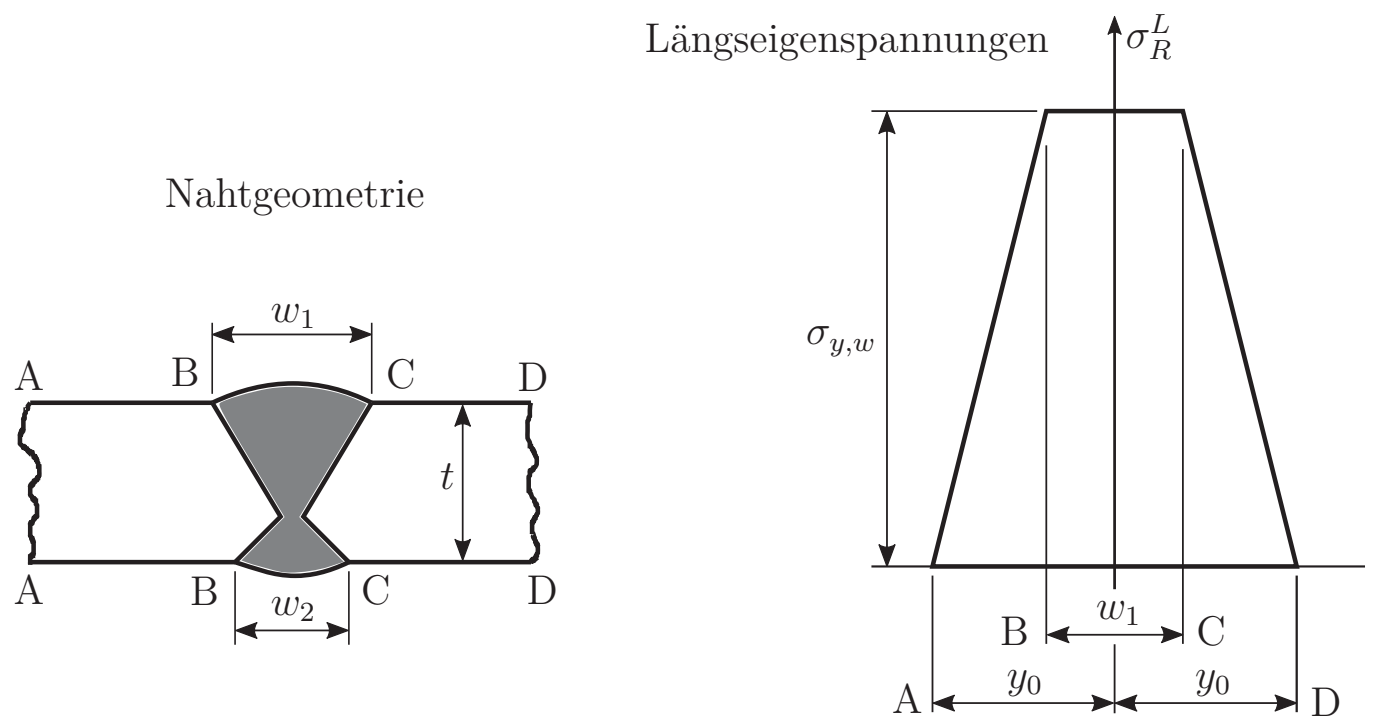

Abb. 6.5.: Schweißeigenspannungen in einer X-Naht nach BARThelEmy (1999)

Das in den 1930er Jahren bevorzugte Schweißverfahren im Stahlbau war das ElektrodenHandschweißen. Für dieses Verfahren ist bei Hänsch (1984) und Neumann (1996) eine durchschnittliche Streckenenergie $q_{s}=61 \mathrm{~J} / \mathrm{mm}^{3}$ Schweißnaht sowie ein mittlerer Wirkungsgrad von $\eta=0,8$ angegeben. Bei einer realisierbaren Lagendicke von $5 \mathrm{~mm}$ [BIERETT (1936), VDEн (1938)] musste die $30 \mathrm{~mm}$ dicke X-Naht zwischen Knotenblech und Zuggurt aus mindestens 2 x 3 Lagen hergestellt werden. Das Überschweißen der jeweils darunter liegenden Lage führte zu einem Normalglühen und damit zu einer Reduzierung der Eigenspannungen. Diese Reduzierung kann nach HÄNSCH (1984) mit dem folgenden Lagenfaktor berücksichtigt werden.

$$
k_{m}=\sqrt[3]{\frac{1}{n^{2}}}=\sqrt[3]{\frac{1}{6^{2}}}=0,3
$$

Bei einer Schweißnahtfläche der überhöhten X-Naht (siehe Abbildung 6.2) von

$$
A_{w}=1,1 \cdot 28 \cdot 15 \approx 460 \mathrm{~mm}^{2}
$$

ergibt sich die Breite der Fließzone zu:

$$
y_{0}=\frac{1,033 \cdot 153}{235} \cdot \frac{0,8 \cdot 0,3 \cdot 61 \cdot 460}{30}=150 \mathrm{~mm}
$$


Die zugehörige Verteilung der Schweißeigenspannungen im Zuggurt in Rissachse ist in Abbildung 6.6 dargestellt. Der Verlauf dieser sekundären Spannungen wird analog zu den primären Spannungen mit einem Polynom 6. Grades approximiert und der Spannungsintensitätsfaktor nach Wu; CARLSSON (1991) ermittelt zu $K_{I}^{s}=29,6 \mathrm{MPa} \sqrt{\mathrm{m}}$

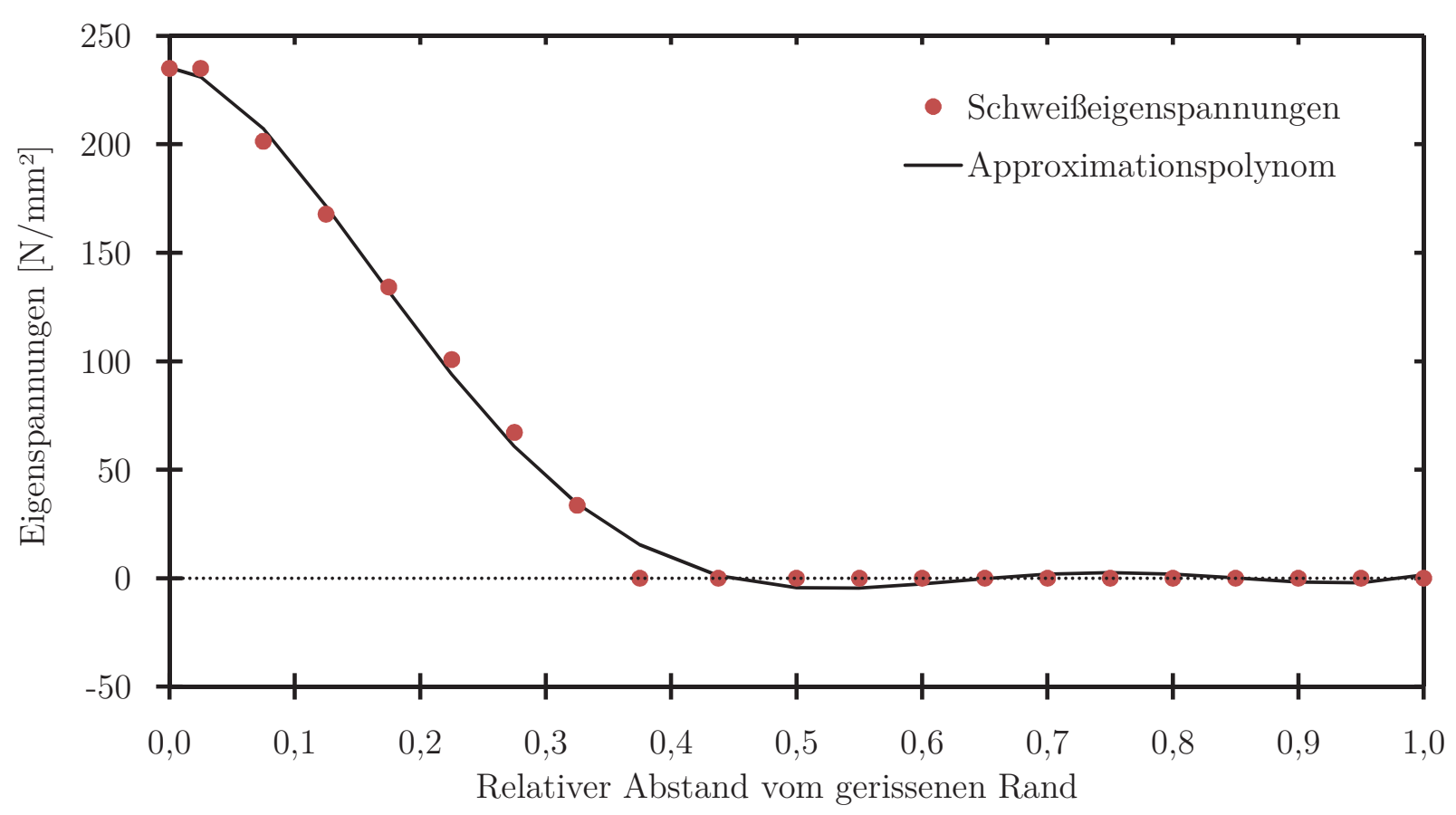

Abb. 6.6.: Approximation der Eigenspannungen im Zuggurt in Rissachse

Während die primären (äußeren) Beanspruchungen einen Einfluss auf den plastischen Kollaps des gerissenen Bauteils haben, stehen die sekundären Spannungen über den Querschnitt im Gleichgewicht. Sie haben aber einen Einfluss auf die Größe der plastizierten Zone an der Rissspitze. Daher ist im Bereich des Kleinbereichfließens (grob $L_{r}<1$ ) die resultierende Rissspitzenbeanspruchung $K_{I}$ größer als die Summe aus $K_{I}^{p}$ und $K_{I}^{s}$. Dieser Effekt wird in SINTAP [BANNISTER; WEBSTER (1999)] durch den Korrekturfaktor $V$ berücksichtigt.

$$
K_{I}=K_{I}^{p}+V \cdot K_{I}^{s}
$$

Dieser Faktor wird berechnet mit:

$$
V=\frac{K_{I p}^{s}}{K_{I}^{s}} \cdot \xi
$$

Hierin ist $K_{I p}^{s}$ der plastizitätskorrigierte K-Faktor für die sekundäre Beanspruchung mit

$$
K_{I p}^{s}=\sqrt{\frac{a_{e f f}}{a}} \cdot K_{I}^{s}
$$

und der plastisch korrigierten Risslänge

$$
a_{e f f}=a+\frac{1}{2 \pi} \cdot\left(\frac{K_{I}^{s}}{R_{e L}}\right)^{2}
$$

Der Faktor $\xi$ liegt z. B. bei ZERBST; SchöDEL et al. (2007) in Abhängigkeit von $L_{r}$ und $K_{I p}^{s} /\left(K_{I}^{p} / L_{r}\right)$ tabelliert vor. Für das untersuchte Detail des angerissenen Zuggurtes ergibt sich:

$$
K_{I}=31,7+1,31 \cdot 29,6=70,3 \mathrm{MPa} \sqrt{\mathrm{m}}
$$


Der Nachweis des Zuggurtes erfolgt nach SINTAP Analyse-Ebene 1A (siehe Abschnitt 2.6.3) im Failure Assessment Diagram. Da keine experimentell ermittelten, mechanischen Kennwerte des Gurtbleches vorliegen, wird für den Nachweis der Mindestwert der Streckgrenze nach Gleichung 5.12 mit $R_{e L}=269 \mathrm{~N} / \mathrm{mm}^{2}$ angesetzt. Dieser gilt bei der für das Bauteil maßgebenden Einsatztemperatur von $-30^{\circ} \mathrm{C}$ nach DIN EN 1993-1-10/NA:2016. Damit ergibt sich die in Abbildung 6.7 dargestellte FAD-Grenzkurve.

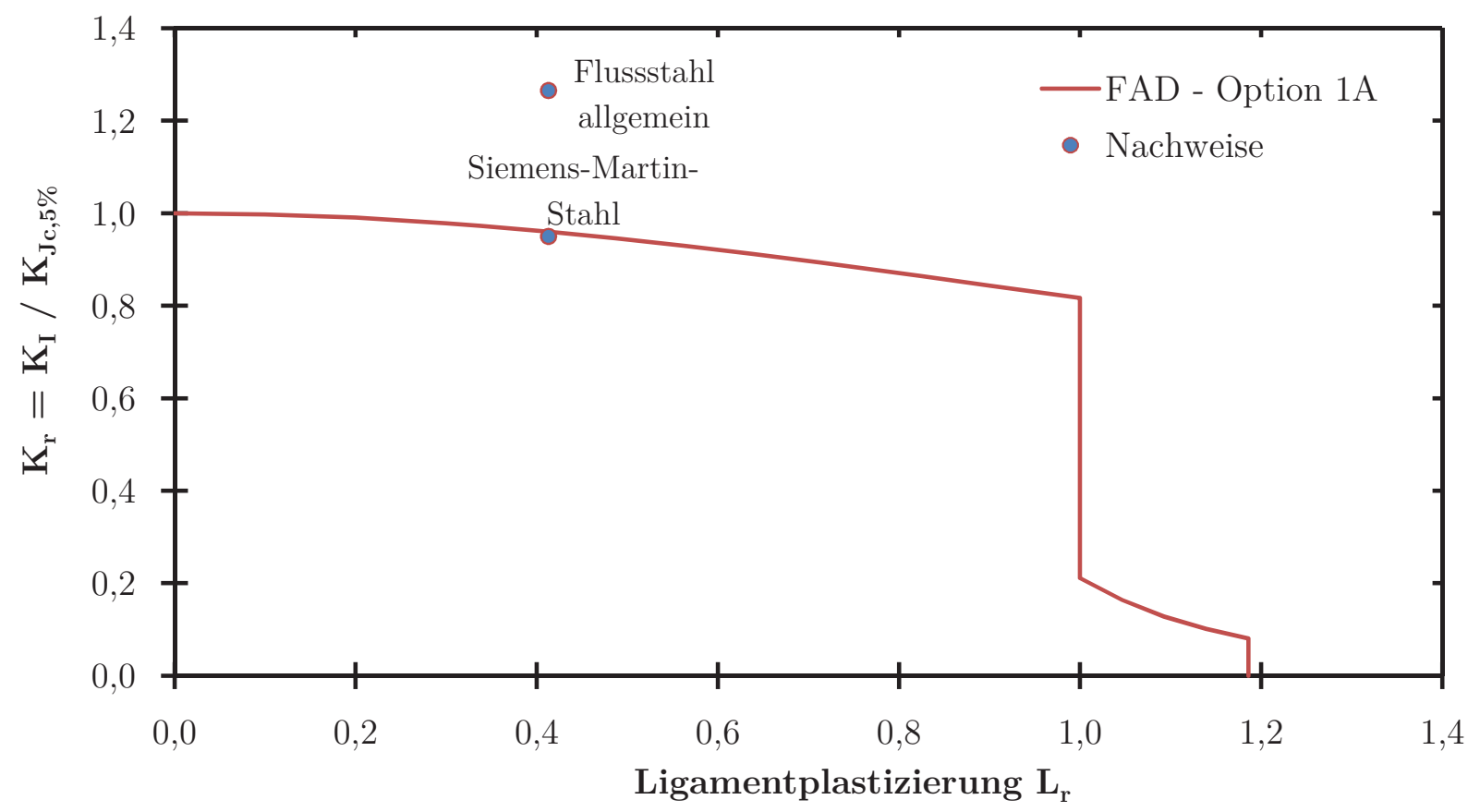

Abb. 6.7.: Nachweis des Zuggurtes im Failure Assessment Diagram

Unter der Annahme, dass ein beliebiger Flussstahl unbestimmter Güte und Werkstoffzähigkeit für den Zuggurt eingesetzt wurde, ergibt sich nach Gleichung 3.25 (Multimodale Master-Curve) eine Bruchzähigkeit von $K_{J c(1 T), 5 \%}=55,6 \mathrm{MPa} \sqrt{\mathrm{m}}$. Ausreichende Sicherheit gegen sprödes Versagen ist mit diesem Grenzwert der Bruchzähigkeit nicht nachweisbar (siehe Abbildung 6.7).

Nach Entnahme einer kleinen Werkstoffprobe am gering beanspruchten Bauteilende des Zuggurtes konnte durch chemische Analyse und metallografisch nachgewiesen werden, dass es sich bei dem Gurtblech um einen Siemens-Martin-Stahl mit geringen Konzentrationen an Phosphor, Schwefel und Stickstoff handelt. Für den Nachweis wird daher die Bruchzähigkeit nach Gleichung $3.26 \mathrm{zu} K_{J c(1 T), 5 \%}=74,1 \mathrm{MPa} \sqrt{\mathrm{m}}$ bestimmt. Der Nachweis gegen sprödes Versagen kann damit erbracht werden.

Der in den Nachweisen berücksichtige Teilsicherheitsbeiwert $\gamma_{M}{ }^{*}=1,03$ zur Abminderung der Bruchzähigkeit basiert auf Untersuchungen von Sтötzel (1998), in denen u. a. Großzugversuche an Bauteilen aus Flussstahl mit symmetrischem Durchriss ausgewertet wurden. 



\section{Kapitel 7.}

\section{Zusammenfassung und Ausblick}

\subsection{Zusammenfassung}

Bei der Beurteilung der Sicherheit bestehender Konstruktionen aus altem Baustahl (i. A. Flussstahl) und der Entscheidung über notwendige Instandsetzungs- und Verstärkungsmaßnahmen ist der Nachweis ausreichender Werkstoffzähigkeit (der Sicherheit gegen ein sprödes Versagen) von wesentlicher Bedeutung. Die in DIN EN 1993-1-10 normativ geregelten Nachweismethoden zur Beurteilung der Sprödbruchgefährdung wurden basierend auf umfangreichen bruchmechanischen Betrachtungen entwickelt. Sie gelten für Schweißkonstruktionen und „Stähle aus neuerer Zeit“ mit in der Regel hohen Zähigkeitswerten. Die Quantifizierung der Werkstoffzähigkeit in DIN EN 1993-1-10 erfolgt durch Kerbschlagbiegeversuche. Die Beziehung zwischen der Übergangstemperatur $T_{27 J}$ der Kerbschlagarbeit und der Referenztemperatur $T_{0}$ der Bruchzähigkeit wird durch die modifizierte Sanz-Korrelation hergestellt, die nur für diese Stähle abgeleitet wurde.

Das in der Norm verankerte Verfahren ist für alte Flussstahlkonstruktionen mit Lochschwächung durch Niet- und Schraubenverbindungen nicht geeignet. Einerseits unterscheiden sich Kerbwirkung und Eigenspannungszustand von geschweißten und genieteten Konstruktionen und damit die Zähigkeitsanforderungen wesentlich voneinander. Auf der anderen Seite unterliegen die Zähigkeitseigenschaften von Flussstählen deutlich größeren Streuungen.

In der vorliegenden Arbeit werden experimentelle, analytische und numerische Untersuchungen zum Sprödbruchverhalten gelochter Konstruktionen aus altem Flussstahl vorgestellt. Wesentlicher Bestandteil sind dabei die umfangreichen Werkstoffanalysen an Bauteilen aus bestehenden Stahltragwerken der Baujahre 1904 bis 1930. Es werden Werkstoffproben aus unterschiedlichen Bauwerken, verschiedenen Querschnittstypen und allen typischen Stahlherstellungsverfahren untersucht. Neben der Ermittlung der Werkstoffkennwerte im Zugversuch wird vor allem die Werkstoffzähigkeit der Stähle analysiert. Dies erfolgt zum einen in instrumentierten Kerbschlagbiegeversuchen zur Ermittlung der Übergangstemperaturen $T_{27 J}$. Zum anderen wird die Bruchzähigkeit $K_{J c}$ in Bruchmechanikversuchen nach ASTM E1820 ermittelt und anschließend die Referenztemperatur $T_{0}$ nach dem Master-Curve-Konzept (ASTM E1921) bestimmt. Angesichts der Inhomogenität der Proben erfolgt unter Einbeziehung aller ermittelten Bruchzähigkeiten eine Auswertung nach dem multimodalen Master-CurveKonzept. Diese ergibt eine Referenztemperatur $T_{0}$ von $-30{ }^{\circ} \mathrm{C}$. Darauf aufbauend wird der charakteristische Wert der Bruchzähigkeit (bei $5 \%$ Bruchwahrscheinlichkeit) in Abhängigkeit von der Einsatztemperatur definiert. 
Bei der Auswertung der Versuchsergebnisse ist die Abhängigkeit der Werkstoffzähigkeit von der chemischen Zusammensetzung des Flussstahls, d.h. der Konzentration ausscheidungsbildender, sprödbruchfördernder Elemente, deutlich erkennbar. Die untersuchten Flussstähle weisen z. T. überdurchschnittlich hohe Bestandteile an Eisenbegleitern auf. Die Probenauswahl bildet daher eine gute Grundlage zur Ermittlung konservativer Werte der Bruchzähigkeit zur Bewertung allgemeiner Flussstähle. Die Ergebnisse werden darüber hinaus anhand existierender Daten zur Bruchzähigkeit von Flussstählen alter Stahlbrücken validiert.

Eine Differenzierung der Bruchzähigkeiten zwischen den tendenziell stärker verunreinigten Konverterstählen (z. B. dem Thomasstahl) und den reineren Siemens-Martin-Stählen ist aus den bisher zur Verfügung stehenden Untersuchungsergebnissen nicht sicher ableitbar. Deshalb werden ergänzende Werkstoffuntersuchungen an Proben aus einer geschweißten Flussstahlkonstruktion durchgeführt. Erwartungsgemäß können alle analysierten Werkstoffe dem Siemens-Martin-Verfahren zugeordnet werden. Die Untersuchungen der Werkstoffzähigkeit, der zum Teil sogar beruhigt vergossenen Flussstähle, ergibt signifikant höhere Bruchzähigkeiten. Die Auswertungen nach dem Master-Curve-Konzept bestätigt die Vermutung, dass in Abhängigkeit des Herstellungsverfahrens unterschiedliche Werkstoffgüten definiert werden können. Für die analysierten Siemens-Martin-Stähle wird eine um ca. $15{ }^{\circ} \mathrm{C}$ tiefere Referenztemperatur $T_{0}$ gegenüber den allgemeinen Flussstählen ermittelt.

Darüber hinaus wird mit Hilfe der für alle Flussstahlproben bestimmten Übergangstemperaturen $T_{27 J}$ eine mögliche Korrelation zur Referenztemperatur $T_{0}$ der Bruchzähigkeit überprüft. Dabei kann nachgewiesen werden, dass die für heutige Baustähle gültige, modifizierte SANZ-Korrelation der Übergangstemperaturen, die Bestandteil der Stahlgütewahl nach DIN EN 1993-1-10 ist, für alte Flussstähle nicht angewendet werden darf. Bei vergleichbaren Referenztemperaturen $T_{0}$ liegt der Übergangsbereich im Kerbschlagbiegeversuch für alte Flussstähle bei deutlich höheren Temperaturen. Für die untersuchten Stähle ergibt sich eine mittlere Differenz der Übergangstemperaturen von $56 \mathrm{~K}$. Eine eindeutige Korrelationsbeziehung zwischen beiden Werkstoffkennwerten ist aus den zur Verfügung stehenden Untersuchungsergebnissen jedoch nicht ableitbar. Unabhängig davon können die bei SINTAP und in der FKM-Richtlinie angegebenen, konservativen Abschätzungen der Bruchzähigkeit aus den Ergebnissen des Kerbschlagbiegeversuchs für alte Flussstähle bestätigt werden.

Um das Anrissverhalten quasi-statisch beanspruchter Bauteile aus Flussstahl, die Werkstoffeigenschaften im Bereich der Loch- und Bauteilränder und deren Einfluss auf die Größe möglicher Anrisse zu untersuchen, werden mechanische und metallografische Untersuchungen an Bauteilen mit gestanzten Löchern und Bauteilkanten durchgeführt. Mit Hilfe von Mikrohärtemessungen kann das Ausmaß der Werkstoffverfestigung durch Stanzen (örtliche Kaltumformung) und Alterung quantifiziert werden. Unabhängig von der Materialdicke beträgt die Tiefe der verfestigten Randzonen bis zu $3 \mathrm{~mm}$.

Durch eine systematische Analyse der konstruktiven Ausbildung verschiedener Stahlfachwerkkonstruktionen des Hochbaus werden typische Querschnitte und Anschlusskonfigurationen identifiziert. Hauptaugenmerk liegt dabei auf den im Stahlhochbau stark verbreiteten Anschlüssen von Winkelprofilen. Unterschieden werden Anschlüsse mit einem und mit mehreren Verbindungsmitteln (Niete oder Schrauben).

Unter Berücksichtigung dieser konstruktiven Randbedingungen und den Erkenntnissen über die Verfestigung an gestanzten Lochrändern, erfolgen umfangreiche bruchmechanische FEMBerechnungen zu Ermittlung der maßgebenden Spannungsintensität $K_{I}$ am Lochrandanriss. 
Im Sinne einer für die Praxis anwendbaren „Handrechnung“ werden die Ergebnisse der FEAnalyse durch Modifikation einer K-Faktor-Lösung aus der Fachliteratur approximiert. Darauf aufbauend wird eine Lösung für Winkelprofilanschlüsse mit mehreren Verbindungsmitteln abgeleitet.

Abschließend werden unter Verwendung der Ergebnisse der Werkstoffuntersuchungen und der numerischen Berechnungen für Winkelprofilanschlüsse Gleichungen zur Ermittlung der Grenzlast, zur Vermeidung von Sprödbruch auf bruchmechanischer Basis, abgeleitet. Um deren Aussagefähigkeit für das damit beschriebene Bauteilversagen zu überprüfen, werden Bauteilzugversuche zur Validierung durchgeführt. Auf Grundlage der experimentell ermittelten Traglasten erfolgt die statistische Auswertung nach Anhang D.8 in DIN EN 1990, um die Bemessungswerte der Anschlusstragfähigkeit sowie die Teilsicherheitsbeiwerte $\gamma_{M}$ für die Anwendung des bruchmechanischen Bemessungsmodells zu bestimmen.

Im Ergebnis der vorliegenden Arbeit steht ein ingenieurmäßig anwendbares Bewertungsverfahren auf semi-probabilistischer Basis zur Verfügung, das dem Anwender die Möglichkeit gibt, eine Sprödbruchbewertung für genietete oder geschraubte Winkelprofilanschlüsse durchzuführen. Darüber hinaus liefern die Erkenntnisse der in dieser Arbeit durchgeführten Werkstoffanalysen wesentliche Grundlagen für die Beurteilung der Sprödbruchneigung alter Flussstähle auf bruchmechanischer Basis.

Die umfangreichen Untersuchungen haben bestätigt, dass die Bewertung alter Flussstähle anhand des Kerbschlagbiegeversuchs, auf Basis der Mindestwerte der Kerbschlagarbeit nach DIN EN 1993-1-10, nicht geeignet ist. Eine sachgerechte Beurteilung der Sprödbruchneigung bestehender Bauwerke aus Flussstahl ist nur auf Basis bruchmechanischer Untersuchungen möglich.

\subsection{Ausblick}

Mit dem Ziel, den Aufwand für die experimentelle Ermittlung der Bruchzähigkeit zu begrenzen, wird ein dreistufiges Verfahren der bruchmechanischen Bewertung der Sprödbruchneigung empfohlen. In der ersten Stufe ist der in dieser Arbeit ermittelte charakteristische Wert der Bruchzähigkeit allgemeiner Flussstähle nach dem Master-Curve-Konzept anzuwenden. Ist damit keine ausreichende Sicherheit gegen Sprödbruch nachweisbar, erfolgt für kritische Bauteile in einer zweiten Untersuchungsstufe die Bestimmung des Herstellungsverfahrens mit Hilfe einfacher chemischer und metallografischer Untersuchungen. Für Bauteile aus gering verunreinigtem Siemens-Martin-Stahl kann der bruchmechanische Nachweis mit dem dafür ermittelten, höheren Wert der Bruchzähigkeit geführt werden. In Bewertungsstufe 3 erfolgt der Nachweis schließlich unter Ansatz der tatsächlichen, experimentell bestimmten Bruchzähigkeit.

Um die für Stufe 2 notwendige Differenzierung der Flussstähle in unterschiedliche Werkstoffgüten zu verifizieren, sind zusätzliche bruchmechanische Untersuchungen an reineren Siemens-Martin-Stählen aber auch an Konverter-Stählen durchzuführen. Diese Untersuchungen sollten von metallografischen Analysen begleitet werden, in denen nach DIN EN ISO 643:2013 die Korngröße des Ferrits im Flussstahl ermittelt und damit der Einfluss der Feinkörnigkeit auf die Werkstoffzähigkeit quantifiziert wird. Ebenso sollte für die analysierten Flussstahlproben der Gehalt nichtmetallischer Einschlüsse nach DIN EN 10247:2007 ermittelt und ein möglicher Zusammenhang zur Werkstoffzähigkeit untersucht werden. Im Falle 
nachweisbarer Korrelationen zwischen diesen Werkstoffeigenschaften und der Bruchzähigkeit, sind die Untersuchungsergebnisse bei der Differenzierung der Güte und Werkstoffzähigkeit alter Flussstähle einzubeziehen.

Die im Rahmen dieser Arbeit durchgeführten Werkstoffuntersuchungen haben bezüglich einer Übergangstemperatur-Korrelation zwischen Kerbschlagarbeit und Bruchzähigkeit gezeigt, dass die für heutige Stähle gültige Korrelation für alte Flussstähle nicht angewendet werden darf. Die Gegenüberstellung der an Flussstahlproben ermittelten Temperaturen $T_{27 J}$ und $T_{0}$ gibt jedoch Grund zu der Annahme, dass ein ähnlicher Zusammenhang ableitbar ist. Um diese Hypothese zu verifizieren oder ggf. zu widerlegen, sind neben den o.g. zusätzlichen bruchmechanischen Untersuchungen auch begleitende Kerbschlagbiegeversuche an Flussstählen unterschiedlicher Güte notwendig.

Wie die Gefügeuntersuchungen an Flussstahlproben mit gestanzten Loch- und Bauteilrändern gezeigt haben, führen die damit verbundene Kaltverfestigung und Reckalterung zu einer deutlichen Festigkeitssteigerung in den betreffenden Randzonen. Während in den in dieser Arbeit betrachteten Berechnungsmodellen von einem Durchreißen dieser Zonen ausgegangen wird, ist auch der unmittelbare Einfluss der Verfestigung auf die Bruchlast und das Anrissverhalten der Bauteile zu untersuchen. Zur experimentellen Ermittlung der Lochrandfestigkeit haben sich tangential entnommene Mikrozugproben aufgrund der geringen Ausdehnung der Randzone nicht bewährt (siehe HuHn (2004)). Besser geeignet sind Schlüsselloch-Proben, wie sie von Linne (2008) zur Untersuchung von oberflächengehärteten Gradientenwerkstoffen eingesetzt wurden. Die Versuchskörper enthalten das komplette, einseitig aufgetrennte Nietloch und werden ähnlich einer C(T)-Probe quasi-statisch beansprucht. Die Zugkraft wird analog zur Messung einer Risswiderstandskurve im Teilentlastungsverfahren aufgebracht. Bei Entlastung verhält sich die Probe rein elastisch. Aus dem Anstieg des linearen Verlaufs im Kraft-Verschiebungs-Diagramm kann damit auf die aktuelle Probensteifigkeit und die Last beim Einreißen der Lochwandung rückgeschlossen werden.

Gemäß der Auswertungen in Abschnitt 2.3 sind neben den in dieser Arbeit untersuchten Winkelprofilanschlüssen auch einschenklige Anschlüsse mit zwei oder mehr Verbindungsmitteln von Bedeutung. Aufbauend auf den in Abschnitt 4.2 beschriebenen FE-Modellen sind hierfür ergänzende numerische Berechnungen durchzuführen und die Ergebnisse durch Erweiterung der Ansätze nach BALL (1987) auf diesen Fall zu approximieren. Die Validierung des Berechnungsmodells erfolgt analog durch Bauteilzugversuche. Sollte, wie im Rahmen dieser Arbeit, die Beschaffung entsprechender Winkelprofile aus Flussstahl mit passender Anschlussgeometrie nicht möglich sein, könnten alternativ Winkel mit nur einem Verbindungsmittel verwendet werden. Die zusätzlich erforderlichen Löcher müssen dann durch Stanzen hergestellt und die Alterungsprozesse der Flussstähle durch künstliche Alterung nachempfunden werden. Um ähnliche Werkstoffeigenschaften wie an den Lochrändern aus der Bauzeit des Tragwerks sicherzustellen, ist das beschriebene Verfahren im Vorfeld durch Gefügeuntersuchungen und Mikrohärtemessungen zu validieren. 


\section{Literatur}

Ainsworth, R. (1984). The Assessment of Defects in Structures of Strain Hardening Material. In: Engineering Fracture Mechanics, Jg. 19 (4), S. 633-642.

Al Laham, S.; Ainsworth, R.; Fox, M. (1998). Stress intensity factor and limit load handbook. FITNET - European Fitness-for-Service Network.

Anderson, T. L. (1995). Fracture mechanics. Fundamentals and applications. 2. Aufl. Boca Raton: CRC Press.

ANSYS (2014). Complete User's Manual. Release 14.5. Canonsburg: ANSYS.

ASTM E 1820:2013. Standard Test Method for Measurement of Fracture Toughness. United States.

ASTM E 1921:2009. Standard Test Method for Determination of Reference Temperature, To, for Ferritic Steels in the Transition Range. United States.

ASTM E 813:1989. Standard Test Method for JIC, A Measure of Fracture Toughness. United States.

BAEHRE, R.; Käpplein, R. (1990). Zeitabhängige Änderungen von Werkstoffeigenschaften bei alten Baustählen. SFB 315 - Erhalten historisch bedeutsamer Bauwerke. Berlin: Ernst und Sohn.

BAll, D. (1987). The Development of Mode I, Linear-Elastic Stress Intensity Factor Solutions for Cracks in Mechanically Fastened Joints. In: Engineering Fracture Mechanics, Jg. 27 (6), S. 653-681.

BAnnister, A. C. (1998). Toughness characterisation of modern structural steels with relevance to European design codes. Properties and in-service performance, Final report. Hrsg. von European Commission. London.

Bannister, A. C.; Webster, S. E. (1999). SINTAP Procedure - Final Version. FITNET - European Fitness-for-Service Network.

Barthelemy, J. Y. (1999). SINTAP - Compendium of residual stress profiles. FITNET European Fitness-for-Service Network.

Beltrami, C. (1995). Numerische Analyse der lokalen und globalen Beanspruchung in Bauteilen mit Rissen und Quantifizierung der Zähigkeitsanforderungen. Dissertation. Berichte aus dem Institut für Eisenhüttenkunde. Aachen: Shaker Verlag.

Berger, C.; Blauel, J. G.; Hodulak, L.; Pyttel, B.; Varfolomeyev, I.; GerDES, C. P. (2006). Bruchmechnischer Festigkeitsnachweis für Maschinenbauteile. FKMRichtlinie. 3. Aufl. Frankfurt (Main): VDMA-Verlag. 
Bierett, G. (1936). Welche Wege weisen die Erkenntnisse über Schrumpfwirkungen den Arbeitsverfahren für die Herstellung von Stumpfnähten im Großstahlbau. In: Stahlbau, Jg. 9 (9), S. 69-71.

Bleich, F. (1932). Stahlhochbauten - Ihre Theorie, Berechnung und bauliche Gestaltung. Erster Band. Berlin: Verlag von Julius Springer.

- (1933). Stahlhochbauten - Ihre Theorie, Berechnung und bauliche Gestaltung. Zweiter Band. Berlin: Verlag von Julius Springer.

Blumenauer, H. (1994). Werkstoffprüfung. 6. Aufl. Leipzig und Stuttgart: Deutscher Verlag für Grundstoffindustrie.

Blumenauer, H.; Pusch, G. (1993). Technische Bruchmechanik. 3. Aufl. Leipzig und Stuttgart: Deutscher Verlag für Grundstoffindustrie.

Böнм, H. (1992). Einführung in die Metallkunde. Bd. 196. B.I.-Hochschultaschenbuch. Mannheim u. a.: BI-Wiss.-Verl.

Braune, H. (1906). Über die Bedeutung des Stickstoffs im Eisen. In: Stahl und Eisen, Jg. 26 $(22,23,24)$, S. 1357-1363, 1431-1437, 1496-1499.

Brown, J. D.; Lubitz, D. J.; Cekov, Y. C.; Frank, K. H.; Keating, P. B. (2007). Evaluation of Influence of Hole Making Upon the Performance of Structural Steel Plates and Connections. CTR Technical Report: 0-4624-1. Austin und Texas.

Brühwiler, E.; Hirt, M. A.; Morf, U.; Huwiler, R. (1989). Bewertung der Spontanbruchgefahr angerissener Brückenbauteile aus Schweißeisen. In: Stahlbau, Jg. 58 (1), S. 9 16.

Brüning, J.; Richter, C.; Seeger, T.; Möll, R.; Jenisch, F.-M.; Germann, J. (2005). Bewertung der Weiterverwendung einer alten Stahlbrücke auf der Grundlage bruchmechanischer Methoden. In: Stahlbau, Jg. 74 (10), S. 754-761.

BS 7910:2005. Guide to methods for assessing the acceptability of flaws in metallic structures. London: British Standards Institution.

Burghardt, H.; Neuhof, G. (1983). Stahlerzeugung. Bedeutung des Siemens-MartinVerfahrens für die Stahlerzeugung der DDR. 1. Aufl. Leipzig: Deutscher Verlag für Grundstoffindustrie.

Cremona, C.; Patron, A.; Johansson, B.; Larsson, T.; Eichler, B.; Höhler, S. (2007). Improved Assessment Methods for Static and Fatigue Resistance of Old Steel Railway Bridges. Sustainable Bridges. Background Document, Final Draft.

DASt-Ri. 009:1973. Empfehlungen zur Wahl der Stahlgütegruppen für geschweißte Stahlbauten. Köln: Stahlbau Verlags- und Service GmbH.

DASt-Ri. 009:2008. Stahlsortenauswahl für geschweißte Stahlbauten. Düsseldorf: Stahlbau Verlags- und Service GmbH.

Deutsche Bahn (2012). DB StationछSService, RB Südost: Übersicht bestehender Stahlbauwerke mit Baujahren vor 1970. unveröffentlicht. Leipzig.

Deutscher Normenausschuss (1941). DIN Taschenbuch 4 - Werkstoffnormen Stahl Eisen Nichteisen-Metalle Technische Lieferbedingungen Eigenschaften Abmessungen. 15. Aufl. Berlin: Beuth. 
- (1952). DIN Taschenbuch 4 Teil A - Werkstoffnormen Stahl und Eisen Technische Lieferbedingungen Eigenschaften, Abmessungen. 18. Aufl. Berlin: Beuth.

- (1959). DIN Taschenbuch 4 A - Werkstoffnormen Stahl und Eisen Technische lieferbedingungen Eigenschaften, Abmessungen. 19. Aufl. Berlin, Köln und Frankfurt/M: Beuth.

DIN 120-2:1936. Berechnungsgrundlagen für Stahlbauteile von Kranen und Kranbahnen Grundsätze für die bauliche Durchbildung. Berlin: Beuth.

DIN 18800-7:2008. Stahlbauten - Ausführung und Herstellerqualifikation. Berlin: Beuth Verlag $\mathrm{GmbH}$.

DIN 50125:2009. Prüfung metallischer Werkstoffe - Zugproben. Berlin: Beuth Verlag GmbH.

DIN EN 10247:2007. Metallographische Prüfung des Gehaltes nichtmetallischer Einschlüsse in Stählen mit Bildreihen. Berlin: Beuth Verlag GmbH.

DIN EN 1090-2:2011. Ausführung von Stahltragwerken und Aluminiumtragwerken - Teil 2: Technische Regeln für die Ausführung von Stahltragwerken. Berlin: Beuth Verlag GmbH.

DIN EN 1990:2010. Eurocode: Grundlagen der Tragwerksplanung. Berlin: Beuth Verlag GmbH.

DIN EN 1993-1-10:2010. Eurocode 3: Bemessung und Konstruktion von Stahlbauten - Teil 1-10: Stahlsortenauswahl im Hinblick auf Bruchzähigkeit und Eigenschaften in Dickenrichtung. Berlin: Beuth Verlag GmbH.

DIN EN 1993-1-10/NA:2016. Eurocode 3: Bemessung und Konstruktion von Stahlbauten Teil 1-10: Stahlsortenauswahl im Hinblick auf Bruchzähigkeit und Eigenschaften in Dickenrichtung - Nationaler Anhang - National festgelegte Parameter. Berlin: Beuth Verlag $\mathrm{GmbH}$.

DIN EN 1993-1-8:2010. Eurocode 3: Bemessung und Konstruktion von Stahlbauten - Teil 1-8: Bemessung von Anschlüssen. Berlin: Beuth Verlag GmbH.

DIN EN ISO 14556:2006. Stahl - Kerbschlagbiegeversuch nach Charpy (V-Kerb) - Instrumentiertes Prüfverfahren. Berlin: Beuth Verlag GmbH.

DIN EN ISO 148-1:2011. Metallische Werkstoffe - Kerbschlagbiegeversuch nach Charpy Teil 1: Prüfverfahren. Berlin: Beuth Verlag GmbH.

DIN EN ISO 643:2013. Stahl - Mikrophotographische Bestimmung der erkennbaren Korngröße. Berlin: Beuth Verlag GmbH.

DIN EN ISO 6507-1:2006. Metallische Werkstoffe - Härteprüfung nach Vickers - Teil 1: Prüfverfahren. Berlin: Beuth Verlag GmbH.

DIN EN ISO 6892-1:2009. Metallische Werkstoffe - Zugversuch - Teil 1: Prüfverfahren bei Raumtemperatur. Berlin: Beuth Verlag GmbH.

DIN ISO 15579:2002. Metallische Werkstoffe - Zugversuch bei tiefen Temperaturen. Berlin: Beuth Verlag GmbH.

Dröge, W.; Bansen, K. (1952). Die Eigenschaften der Thomas-, Siemens-Martin- und Elektro-Stähle. Bd. 48. Wissenschaftliche Berichte : Folge 1, Metallurgie und Giessereiwesen. Berlin: Verl. Technik.

Dugdale, D. (1960). Yielding of steel sheets containing slits. In: Journal of the Mechanics and Physics of Solids, Jg. 8 (2), S. 100-104. 
ECKART, K. (1988). Die Eisen- und Stahlindustrie in den beiden deutschen Staaten. Bd. 87. Erdkundliches Wissen. Stuttgart: Steiner-Verlag.

EHRHARDT, H. (1988). Untersuchungen zum Einfluß unterschiedlicher Fehlergeometrien auf das Versagensverhalten von Stahl auf der Grundlage von Großzugversuchen. Dissertation. RWTH Aachen.

Forman, R.; Shivakumar, V.; Mettu, S.; Beek, J.; Williams, L.; Yeh, F. (2002). NASGRO - Fracture Mechanics and Fatigue Crack Growth Analysis Software. Reference Manual, Version 4.02. Houston und Texas: NASA Johnson Space Center und Southwest Research Institute.

FreitaG, A. (2013). Quantifizierung der erforderlichen Bruchzähigkeit von Winkelprofilen zur Vermeidung von Sprödbruch. Diplomarbeit. TU Dresden: Institut für Stahl- und Holzbau.

GeISSLER, K. (1995). Beitrag zur probabilistischen Berechnung der Restnutzungsdauer stählerner Brücken. Dissertation. TU Dresden: Institut für Tragwerke und Baustoffe.

GöBel, A.; Henkel, O. (1920). Grundzüge des Eisenbaus (Eisenkonstruktionen - Teil II). Leitfaden für den Unterricht an Baugewerbeschulen und verwandten technischen Lehranstalten. 4. umgearbeitete und vermehrte Auflage. Leipzig und Berlin: B.G. Teubner.

- (1921). Grundzüge des Eisenbaus (Eisenkonstruktionen - Teil I). Leitfaden für den Unterricht an Baugewerbeschulen und verwandten technischen Lehranstalten. 5. Auflage. Leipzig und Berlin: B.G. Teubner.

Gräfen, H. (1991). Lexikon Werkstofftechnik. Düsseldorf: VDI-Verlag.

Groth, C.; Müller, G. (2009). FEM für Praktiker. Basiswissen und Arbeitsbeispiele zu FEM-Anwendungen. 5. Aufl. Edition expertsoft. Renningen-Malmsheim: expert-Verlag.

Hanemann, H. (1911). Hütte. Des Ingenieurs Taschenbuch. 21. Aufl. Bd. 1. 3 Bde. Berlin: Verlag von Wilhelm Ernst \& Sohn.

Hänsch, H. (1984). Schweißeigenspannungen und Formänderungen an stabartigen Bauteilen. Berechnung u. Bewertung. 1. Aufl. Betriebspraxis. Berlin: Verl. Technik.

Helmerich, R. (2005). Alte Stähle und Stahlkonstruktionen. Materialuntersuchungen, Ermüdungsversuche an originalen Brückenträgern und Messungen von 1990 bis 2003. Berlin: Bundesanst. für Materialforschung und -prüfung.

Helms, R.; Kühn, H.-D.; Martin, E. (1973). Sprödbruchsicherheit von Stahlbauteilen mit gestanzten Löchern. Mitteilung aus der Bundesanstalt für Materialprüfung (BAM), Berlin. In: Archiv für das Eisenhüttenwesen, Jg. 44 (12), S. 917-926.

Hensen, W. (1992). Grundlagen für die Beurteilung der Weiterverwendung alter Stahlbrücken. Dissertation. Bd. 21. Schriftenreihe Lehrstuhl für Stahlbau. RWTH Aachen.

Hobbacher, A. (2008). Recommendations for Fatigue Design of welded Joints and Components. Hrsg. von IIW Commissions XIII AND XV. Paris.

Huhn, H. (2004). Ermüdungsfestigkeit von Schraubenverbindungen aus feuerverzinkten Stahlbauteilen mit gestanzten Löchern. Fortschrittberichte VDI : Reihe 4, Bauingenieurwesen. Düsseldorf: VDI-Verlag.

ISO 15653:2010. Metallic materials - Method of test for the determination of quasistatic fracture toughness of welds - First edition. Genf. 
JCSS (2001a). Probabilistic Model Code. Part 3 - Resistance Models, 3.10 Dimensions. Hrsg. von Joint Committee on Structural Safety.

- (2001b). Probabilistic Model Code. Part 3 - Resistance Models, 3.02 Structural Steel. Hrsg. von Joint Committee on Structural Safety.

Käpplein, R.; Wielgosch-Frey, A. (2001). Historische Eisen- und Stahlkonstruktionen. Untersuchen, Berechnen, Instandsetzen. Karlsruhe: Sonderforschungsbereich 315 Univ. Karlsruhe.

Klinger, C.; Mehdianpour, M.; Klingbeil, D.; Bettge, D.; HÄcker, R.; Baer, W. (2011). Failure analysis on collapsed towers of overhead electrical lines in the region Münsterland (Germany) 2005. In: Engineering Failure Analysis Journal, Jg. 18 (7), S. $1873-1883$.

KüHN, B. (2005). Beitrag zur Vereinheitlichung der europäischen Regelungen zur Vermeidung von Sprödbruch. Dissertation. Aachen: Shaker Verlag.

Kühn, B.; Lukić, M.; Nussbaumer, A.; Günther, H.-P.; Helmerich, R.; Herion, S.; Kolstein, M.; Walbridge, S.; Androic, B.; Dijkstra, O.; Bucak, Ö. (2008). Assessment of Existing Steel Structures: Recommendations for Estimation of Remaining Fatigue Life. Background documents in support to the implementation, harmonization and further development of the Eurocodes.

Kumar, V.; German, M.; Shin, C. (1981). An Engineering Approach for Elastic-Plastic Fracture Analysis. NP-1931 Research Project 1237-1. Hrsg. von GENERAL ELECTRIC COMPANY - Corporate Research And Development. New York.

Kuna, M. (2010). Numerische Beanspruchungsanalyse von Rissen. Finite Elemente in der Bruchmechanik. 2. Aufl. Wiesbaden: Vieweg + Teubner.

Kuscher, G. (2012). Sanierung alter Stahlkonstruktionen - Wann ist Altstahl schweißgeeignet? Zukunftsorientierte Stahllösungen für das Bauen im Bestand. Denkmal - Europäische Messe für Denkmalpflege, Restaurierung und Altbausanierung. Hrsg. von BAUforumSTAHL E.V. Leipzig.

Landes, D.; Heerens, J.; Schwalbe, K.-H.; Petrovski, B. (1993). Size, Thickness and Geometry Effects on Transition Fracture. In: Fatigue and Fracture of Engineering Materials and Structures, Jg. 16, S. 1135-1146.

Landes, D.; Shaffer, D. H. (1980). Statistical Characterization of Fracture in the Transition Region. In: ASTM STP 700. Hrsg. von ASTM InTERnationaL. West Conshohocken und PA, S. 368-382.

LAngenBerG, P. (1996). Bruchmechanische Sicherheitsanalyse anrissgefährdeter Bauteile im Stahlbau. Dissertation. Berichte aus dem Institut für Eisenhüttenkunde. Aachen: Shaker.

Larsson, T. (2009). Fatigue assessment of riveted bridges. Doctoral Thesis. Luleå: Luleå University of Technology.

Linne, K. (2008). Untersuchungen des Anriss- und Rissfortschrittverhaltens von Bauteilen aus Gradientenwerkstoffen nach statischen Überlasten. Dissertation. Bd. 82. Veröffentlichung des Instituts für Stahlbau und Werkstoffmechanik der Technischen Universität Darmstadt. Darmstadt: Inst. für Stahlbau und Werkstoffmechanik. 
LüDDECKE, F. (2006). Ein Beitrag zur Ertüchtigung bestehender Stahltragwerke unter besonderer Berücksichtigung des Fügeverfahrens Schweißen. Dissertation an der TU Dresden. Bd. 17. BAM Dissertationsreihe. Bremerhaven: Verlag für neue Wissenschaft GmbH.

Madia, M.; Arafah, D.; Zerbst, U. (2014). Reference load solutions for plates with semielliptical surface cracks subjected to biaxial tensile loading. In: International Journal of Pressure Vessels and Piping, Jg. 119, S. 19-28.

Mang, F.; Bucak, Ö. (1996). Tauglichkeit und Lebensdauer von bestehenden Stahlbauwerken. In: Stahlbau-Handbuch. Für Studium und Praxis. Hrsg. von Deutscher StahlBauVerband. 3. Aufl. Bd. 1B. 1B. Köln: Stahlbau-Verlagsgesellschaft mbH, S. 125-250.

Margolin, B. Z.; Gulenko, A. G.; Nikolaev, V. A.; Ryadkov, L. N. (2005). Prediction of the Dependence of KJC(T) on neutron Fluence for RPV Steels on Basis of the Unified Curve Concept. In: International Journal of Pressure Vessel and Piping, Jg. 82 (9), S. 679-686.

Meyendorf, M. (1999). Korrelationen zwischen Kerbschlagarbeit und Bruchzähigkeit. Studienarbeit. Darmstadt: MPA/IfW.

Michels, C.; Hagedorn, K. (1997). Vergleich der Beurteilung der Zähigkeitsanforderung zur Vermeidung von Sprödbruch nach konventionellen Prüfverfahren und nach der Bruchmechanik. Gebrauchseigenschaften und Betriebsverhalten, Abschlußbericht. Hrsg. von EuROPÄIsche Kommission. Düsseldorf.

Michels, C. (1995). Beurteilung der Zähigkeitsanforderung zur Vermeidung von Sprödbruch nach konventionellen Prüfverfahren und nach der Bruchmechanik. Dissertation. Umformtechnische Schriften. Düsseldorf: Verlag Stahleisen.

Miki, C.; Fukazawa, M.; Katoh, M.; Ohune, H. (1989). Feasibility study on nondestructive methods for fatigue crack detection in steel bridge members. In: Welding in the World, Jg. 27 (9/10), S. 248-266.

MöLl, R. (1997). Altstahlschweißen und Nieten im Zuge der Grunderneuerung des "Eisernen Steges" in Frankfurt am Main. In: Stahlbau, Jg. 66 (1), S. 1-11.

Moreno, J.; VAliente, A. (2007). J-integral determination in riveted beams of a structural old steel by estimation methods. In: Fatigue \& Fracture of Engineering Materials and Structures, Jg. 30 (8), S. 712-722.

Mors, H. (1980). Erfahrungen auf Prüfstationen für Freileitungsmaste. In: Stahlbau, Jg. 49 (6), S. 161-165.

Müller, K.; Pusch, G. (2001). Anwendung des instrumentierten Kerbschlagbiegeversuchs zur Abschätzung der Rissauffangzähigkeit. In: Materialwissenschaft und Werkstofftechnik, Jg. 32, S. 539-543.

Murakami, Y. (1990). Stress intensity factors handbook. Volume 1. (Committee on Fracture Mechanics, The Society of Materials Science, Japan, Ed.-in-chief: Y. Murakami). Oxford: Pergamon Press.

National Cooperative Highway Research Program Report (1987). Fatigue and Fracture Evaluation for Rating Riveted Bridges. Report 302. Washington D.C.: National Research Council. 
Naumann, F. K. (1961). Die Versprödung der Kanten beim Scherenschnitt von Grobblechen aus Thomas- und Siemens-Martin-Stahl. Teil I : Betriebsversuche. In: Stahl und Eisen, Jg. 81 (21), S. 1404-1418.

Neumann, A. (1996). Schweisstechnisches Handbuch für Konstrukteure. 7. Aufl. Bd. 80. Fachbuchreihe schweisstechnik. Düsseldorf: Deutscher Verlag für Schweisstechnik DVSVerlag.

Newman, J. C. (1971). An improved method of collocation for the stress analysis of cracked plates with various shaped boundaries. Hrsg. von LAngley ReSEARCH CEnTER. Hampton VA.

Niessen, T. (1994). Statistische Auswertung der Festigkeits- und Zähigkeitseigenschaften von Flußstählen und Puddelstählen aus älteren Brückenkonstruktionen. Institut für Eisenhüttenkunde. Diplomarbeit. Aachen: Rheinisch-Westfälischen Technischen Hochschule.

Petzow, G. (1976). Metallographisches Ätzen. Hrsg. von Schrader, Angelica. 5. Aufl. Materialkundlich-technische Reihe. Berlin: Bornträger.

PhaAl, R.; Macdonald, K. A. (1991). Critical examination of correlations between fracture toughness and charpy impact energy. Report - OTO 96 710. Hrsg. von THE WeLDING Institute. Cambridge.

REICHE, A. (2000). Zustandsbewertung von metallischen Tragwerkskomponenten. Stuttgart: Fraunhofer-IRB-Verl.

Rice, J. R. (1968). A Path Independent Integral and the Approximate Analysis of Strain Concentration by Notches and Cracks. In: Journal of Applied Mechanics, Jg. 35 (2), S. 379 386.

Rooke, D.; Tweed, J. (1980). Stress Intensity Factors for a Crack at the Edge of a Pressurized Hole. In: International Journal of Engineering Science, Jg. 18 (1), S. 109-121.

Roos, W. (2005). Das RWE-Netz - Fakten. Präsentation nach den Strommasteinstürzen im Münsterland. Berlin: Vorstand Ressort Netze RWE Energy AG.

SANZ, G. (1980). Essai de mise au point dune method quantitative de choix des qualities d'aciers vis-à-vis du risqué de rupture fragile. In: Revue de Métallurgie, S. 621-642.

Sarja, A. (2002). Neue Anforderungen an die Dauerhaftigkeit von Konstruktionen. In: Der Prüfingenieur (20), S. 46-53.

Schatt, W.; Simmchen, E.; Zouhar, G. (1998). Konstruktionswerkstoffe des Maschinenund Anlagenbaues. 5. Aufl. Stuttgart: Deutscher Verlag für Grundstoffindustrie.

Schulze, G. (2010). Die Metallurgie des Schweissens. Eisenwerkstoffe - Nichteisenmetallische Werkstoffe. 4. Aufl. VDI-Buch. Berlin und Heidelberg: Springer-Verlag.

Schwalbe, K. H. (1986). The Crack Tip Opening Displacement in Elastic-Plastic Fracture Mechanics. Proceedings of the Workshop on the CTOD Methodology GKSS Forschungszentrum Geesthacht, Germany, April 23-25, 1985. Berlin und Heidelberg: Springer Verlag.

SchwalBE, K.-H. (1980). Bruchmechanik metallischer Werkstoffe. München: Hanser Verlag.

Sedlacek, G.; Feldmann, M.; Kühn, B.; Tschickardt, D.; Höhler, S.; Müller, C.; Hensen, W.; Stranghöner, N.; Dahl, W.; Langenberg, P.; Münstermann, S.; Brozetti, J.; Raoul, J.; Pope, R.; Bijlaard, F. (2008). Commentary and Wor- 
ked Examples to EN 1993-1-10 "Material toughness and through thickness properties" and other toughness oriented rules in EN 1993. Background documents in support to the implementation, harmonization and further development of the Eurocodes. Joint Report Prepared under the JRC - ECCS cooperation agreement for the evolution of Eurocode 3. Hrsg. von JRC European Commission. Version First Edition.

Sedlacek, G.; Hensen, W.; Bild, J.; Dahl, W.; Langenberg, P. (1992). Verfahren zur Ermittlung der Sicherheit von alten Stahlbrücken unter Verwendung neuester Erkenntnisse der Werkstofftechnik. In: Bauingenieur, Jg. 67, S. 129-136.

SES:1953. Standardliste Eisen und Stahl. Berlin: Die Wirtschaft.

Shin, C. F.; Moran, B.; NAkAmura, T. (1986). Energy release rate along a three dimensional crack front in a thermally stressed body. In: International Journal of Fracture, Jg. 30 (2), S. 79-102.

SILCHER, H. (1991). Untersuchung der Einflußfaktoren auf das bruchmechanische Verhalten von Proben mit symmetrischen und asymmetrischen Fehlergeometrien. Dissertation. Stuttgart.

Silva, L. S. da; Tankova, T.; Marques, L.; Rebelo, C. (2015). Savety Assessment of EC3 Stability Design Rules for flexural Buckling of Columns. Meeting of Working Group 1 EC3 CEN TC 250-SC3-WG1. Hrsg. von ISISE - DeparTMENT of CiviL Engineering, University of Coimbra Portugal.

Spaethe, G. (1992). Die Sicherheit tragender Baukonstruktionen. 2. Aufl. Wien und New York: Springer Verlag.

Steguweit, C. (1998). Neue Bahnhofskonzeptionen, Eisenbahn und Denkmalpflege. Drittes internationales Eisenbahnsymposium des Deutschen Nationalkomitees von ICOMOS, Frankfurt am Main, 14. bis 16. April 199\%. Bd. 27. ICOMOS-Hefte des Deutschen Nationalkomitees ICOMOS-journals of the German National Committee. München: K.M. Lipp.

Steinhardt, O. (1954). Über das Stanzen von Blechen. Bericht aus der Versuchsanstalt für Stahl, Holz und Steine. Hrsg. von Technische Hochschule Karlsruhe.

Stötzel, G. (1998). Verfahren zur Beurteilung der Sicherheit bei Weiterverwendung alter Stahlbrücken. Dissertation. Bd. 39. Stahlbau. Aachen: Shaker Verlag.

Stroetmann, R.; Flederer, H.; Schmidt, H.; Sieber, L. (2009). Personenaufzug Bad Schandau - Revitalisierung eines denkmalgeschützten Turmbauwerks. In: Stahlbau, Jg. 78 (9), S. 662-676.

Stroetmann, R.; Sieber, L.; Viehrig, H.-W.; Houska, M.; Vetter, B.; Schubert, V. (2015). Beurteilung der Sprödbruchgefährdung gelochter Stahltragwerke - Weiterentwicklung der Analysemethoden. Forschungsinitiative Zukunft Bau, Aktenzeichen: II 3F20-12-1-054. Stuttgart: Fraunhofer-IRB-Verlag.

Stromeyer, C. E. (1910). Stickstoff als Ursache der Sprödigkeit bei weichem Stahl. In: Stahl und Eisen, Jg. 30, S. 1805.

Tada, H.; Paris, P. C.; Irwin, G. R. (2000). The Stress Analysis of Cracks Handbook. 3. Aufl. New York: ASME Press. 
TASCHe, M. (2015). Analyse von Entwicklungssträngen im Konstruktiven Ingenieurbau anhand bestehender Brücken und Stabtragwerke im Hochbau in Sachsen, Sachsen-Anhalt und Thüringen. Dissertation. TU Dresden.

TGL 7960:1960. Allgemeine Baustähle Gütebedingungen. Leipzig: VEB Fachbuchverlag.

TGL 7960:1975. Allgemeine Baustähle, Stahlmarken, Allgemeine technische Forderungen. Berlin: Staatsverlag der DDR.

Tweed, J.; Rooke, D. (1979). The Stress Intensity Factor for a Crack at the Edge of a Loaded Hole. In: International Journal of Solids and Structures, Jg. 15 (11), S. 899-906.

Ungermann, D. (2008). Handbuch historischer Stahlhochbaukonstruktionen (1880-1940). Abschlussbericht (unveröffentlicht). Hrsg. von Technische Universität Dortmund. Dortmund.

Valtinat, G.; Dangelmaier, P. (1993). Schraubenverbindungen mit gestanzten Löchern in zugbeanspruchten, feuerverzinkten Bauteilen. Forschungsvorhaben GAV-Nr. FD 18, AiF-Nr. 7448. Hrsg. von Gemeinschaftsausschuss Verzinken e.V. Düsseldorf.

VDE-AR-N 4210-3:2011. Prüf- und Bewertungsverfahren zu Ermittlung der Tragfähigkeit von Bauteilen aus Thomasstahl in Stahlgitter-Freileitungsmasten mit Nennspannungen ab 110 $k V$. Berlin: VDE.

Verein Deutscher Eisenhüttenleute (1889a). Gemeinfassliche Darstellung des Eisenhüttenwesens. 1. Aufl. Düsseldorf: Kommissionsverlag von A. Bagel.

- (1889b). Vorschriften für die Lieferung von Eisen und Stahl. Düsseldorf: Commissionsverlag von August Bagel.

- (1907). Gemeinfassliche Darstellung des Eisenhüttenwesens. 6. Aufl. Düsseldorf: Kommissionsverlag von A. Bagel.

- (1929). Gemeinfassliche Darstellung des Eisenhüttenwesens. 13. Aufl. Düsseldorf: Verlag Stahleisen.

- (1930). Stahl im Hochbau / Taschenbuch für Entwurf, Berechnung u. Ausführung von Stahlbauten. 8. Aufl. Düsseldorf: Verlag Stahleisen.

- (1937). Gemeinfassliche Darstellung des Eisenhüttenwesens. 14. Aufl. Düsseldorf: Verlag Stahleisen.

- (1938). Stahl im Hochbau / Taschenbuch für Entwurf, Berechnung u. Ausführung von Stahlbauten. 10. Aufl. Düsseldorf: Verlag Stahleisen.

- (1971). Gemeinfassliche Darstellung des Eisenhüttenwesens. 17. Aufl. Düsseldorf: Verlag Stahleisen.

VERSCH, C. (2014). Ein Beitrag zur Vermeidung von Sprödbrüchen bei nicht durchgeschweißten Verbindungen im Stahlbau. Dissertation. Darmstadt: Inst. für Stahlbau und Werkstoffmechanik.

Wallin, K. (1984). The Scatter in KIC-Results. In: Engineering Fracture Mechanics, Jg. 19 (6), S. 1085-1093.

- (1985). The Size Effect in KJc Results. In: Engineering Fracture Mechanics, Jg. 22 (1), S. 149-163. 
Wallin, K. (1998). Master Curve Analysis of Ductile to Brittle Transition Region Fracture Toughness Round Robin Data - The 'Euro' Fracture Toughness Curve. Bd. 367. VTT Publications. Espoo: Technical Research Centre of Finland.

Wallin, K.; Nevasmaa, P.; Laukkanen, A.; Planman, T. (2004). Master Curve analysis of inhomogeneous ferritic steels. In: Engineering Fracture Mechanics, Jg. 71 (16-17), S. 2329-2346.

Wanlin, G. (1993). Stress Intensity Factors for Corner Cracks at Holes subjected to biaxial and Pin Loads. In: Engineering Fracture Mechanics, Jg. 46 (3), S. 473-479.

Werner, F.; SeIdel, J. (1992). Der Eisenbau. Vom Werdegang einer Bauweise. Berlin und München: Verlag für Bauwesen.

Wienert, H. (1992). Die Stahlindustrie in der DDR. Bd. 52. Schriftenreihe des RheinischWestfälischen Instituts für Wirtschaftsforschung, Essen. Berlin: Duncker \& Humblot.

Wittek, K. H. (1964). Die Entwicklung des Stahlhochbaus. Von den Anfängen (1800) bis zum Dreigelenkbogen (18\%0). Dissertation. Beiträge zur Technikgeschichte. Düsseldorf: VDI-Verlag.

Wittemann, K.; BaeHRe, R. (1993). Bruchmechanische Untersuchungen an Baustahl des 19. Jahrhunderts. Jahrbuch Sonderforschungsbereich 315. Erhalten historisch bedeutsamer Bauwerke. Ernst und Sohn.

WTA E-7-3-05/D:2005. Sanierung Historischer Stahl- und Gusskonstruktionen nach WTA. München: WTA.

Wu, X.-R.; Carlsson, J. (1991). Weight functions and stress intensity factor solutions. 1. Aufl. Oxford und New York: Pergamon Press.

Zerbst, U.; Wiesner, C.; Kocak, M.; Hodulak, L. (1999). SINTAP: Entwurf einer vereinheitlichten europäischen Fehlerbewertungsprozedur - eine Einführung. Hrsg. von GKSS-ForschungSZENTRUM GEESTHACHT.

Zerbst, U.; Ainsworth, B.; Madia, M. (2010). Grenzlast oder Referenzlast? Vorschlag für eine hybride Vorgehensweise bei der elastisch-plastischen Bauteilbewertung. Unter Mitarb. von DVM. Paderborn.

Zerbst, U.; Schödel, M.; Webster, S.; Ainsworth, R. (2007). Fitness-for-service fracture assessment of structures containing cracks. A workbook based on the European SINTAP/FITNET procedure. 1. Aufl. Amsterdam und Heidelberg: Elsevier Academic Press. 
Anhang A.

Darstellungen der Ergebnisse aus den Werkstoffuntersuchungen 


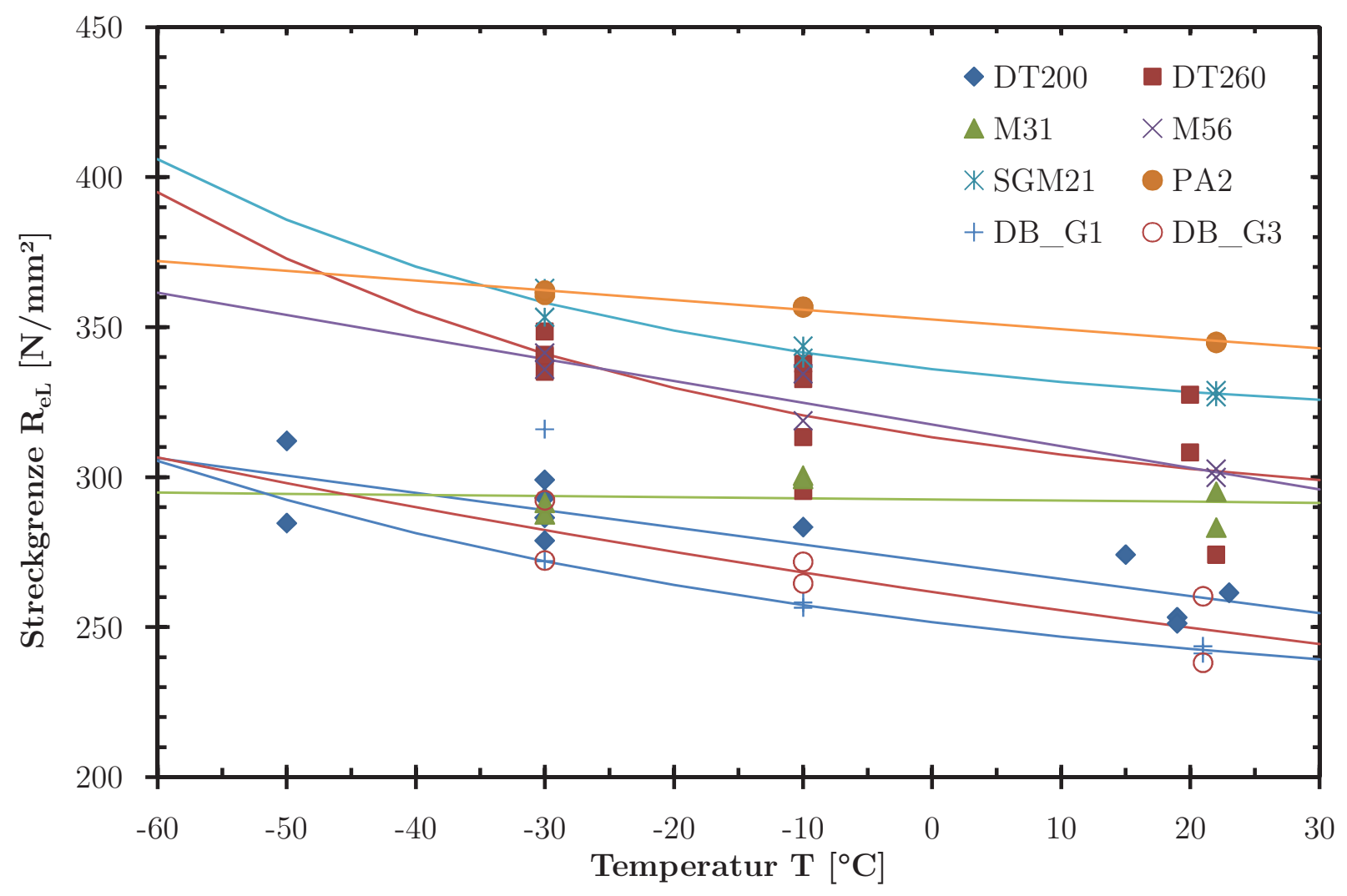

Abb. A.1.: Untere Streckgrenze $R_{e L}$ in Abhängigkeit der Temperatur

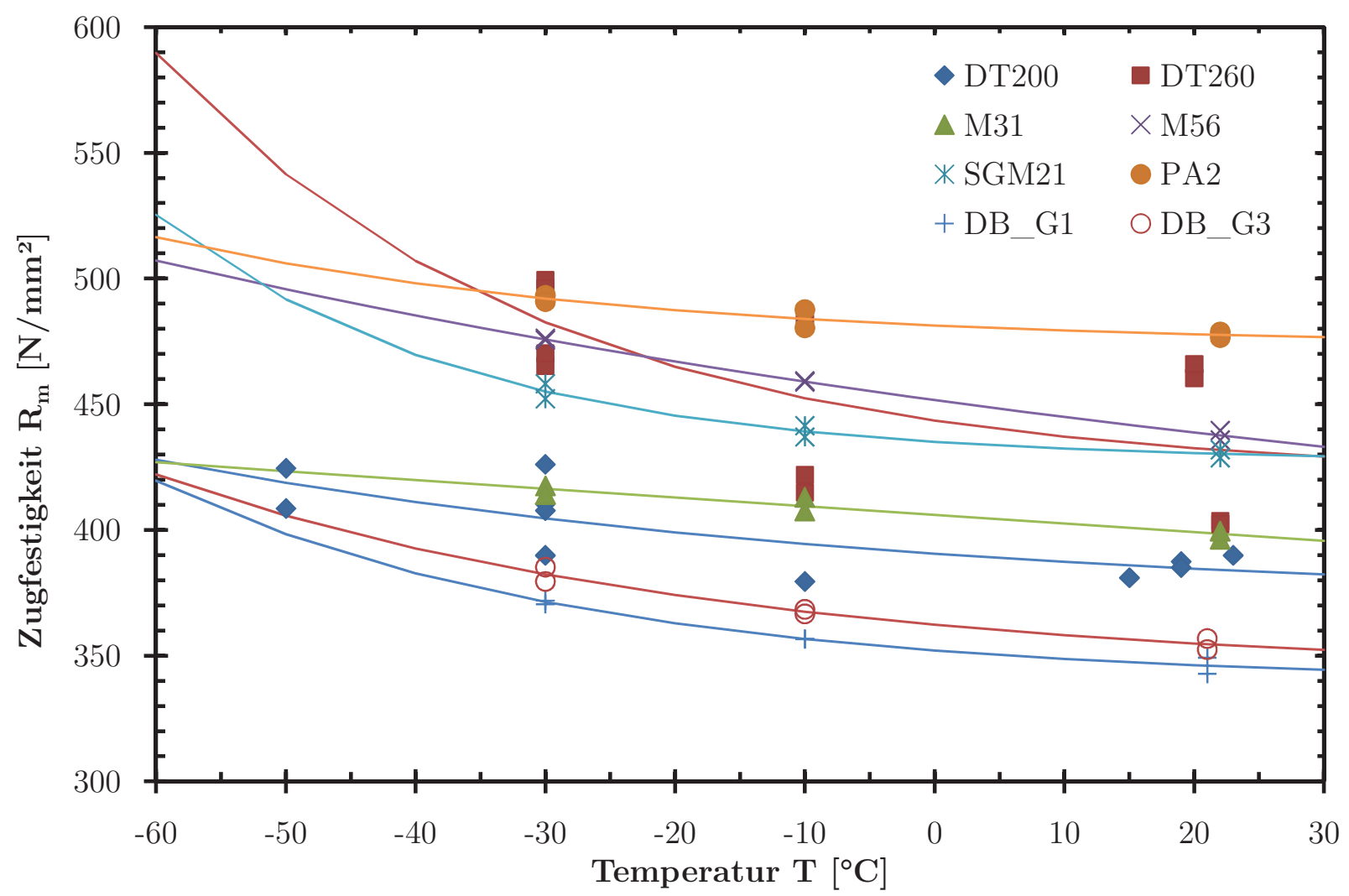

Abb. A.2.: Zugfestigkeit $R_{m}$ in Abhängigkeit der Temperatur 


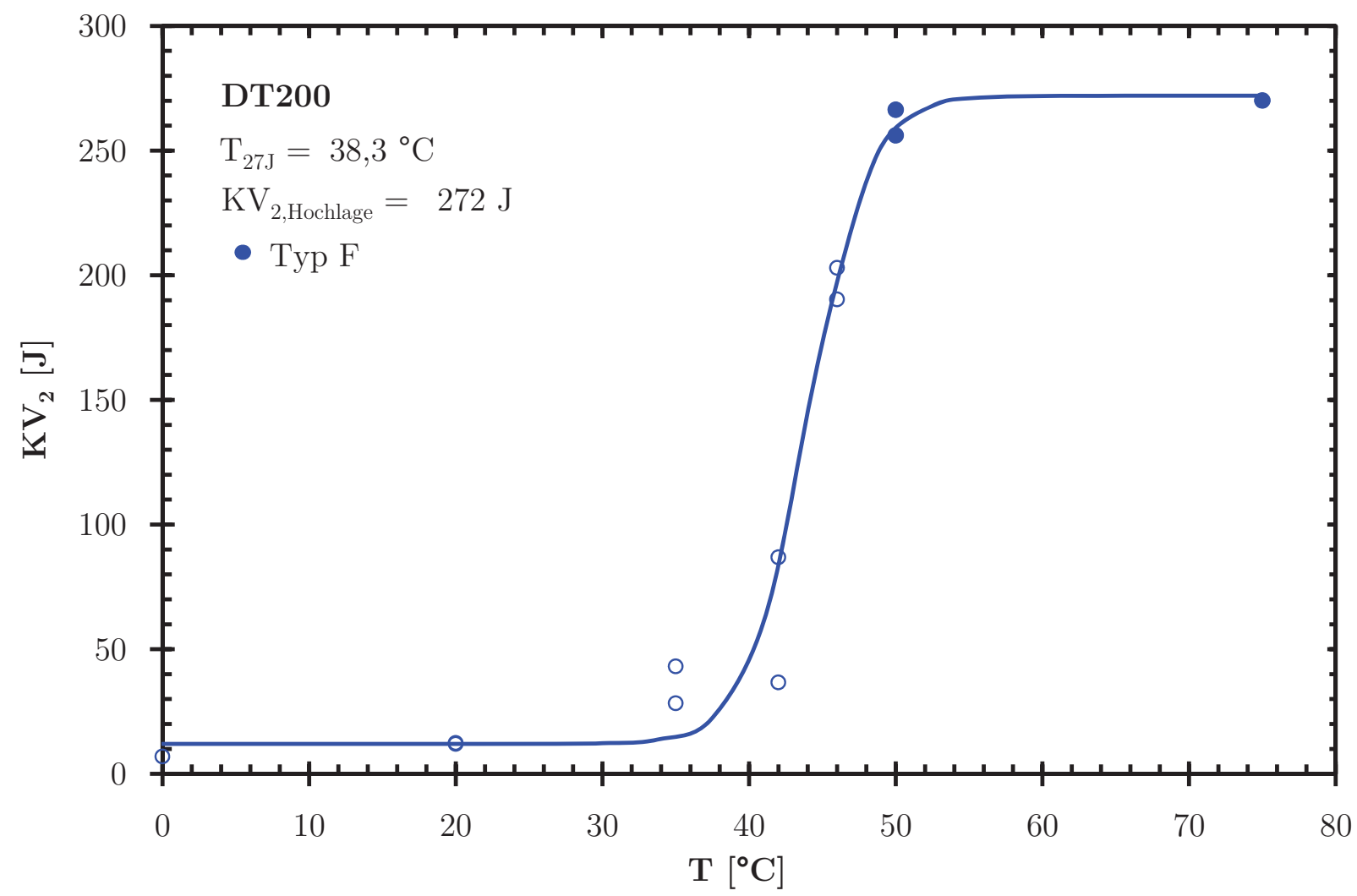

Abb. A.3.: Schlagarbeiten und Kerbschlag-Temperaturkurve der Probe DT200

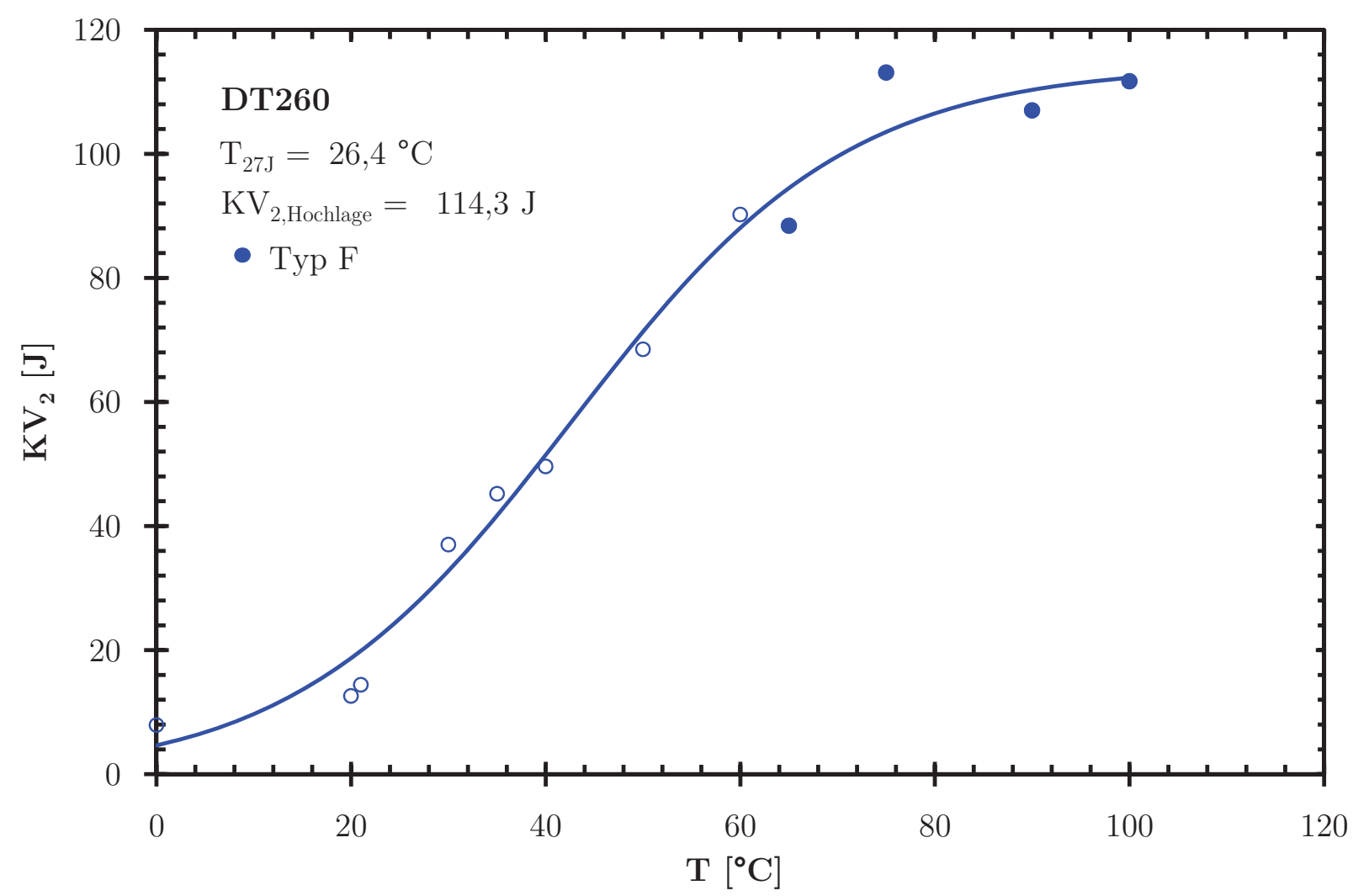

Abb. A.4.: Schlagarbeiten und Kerbschlag-Temperaturkurve der Probe DT260 


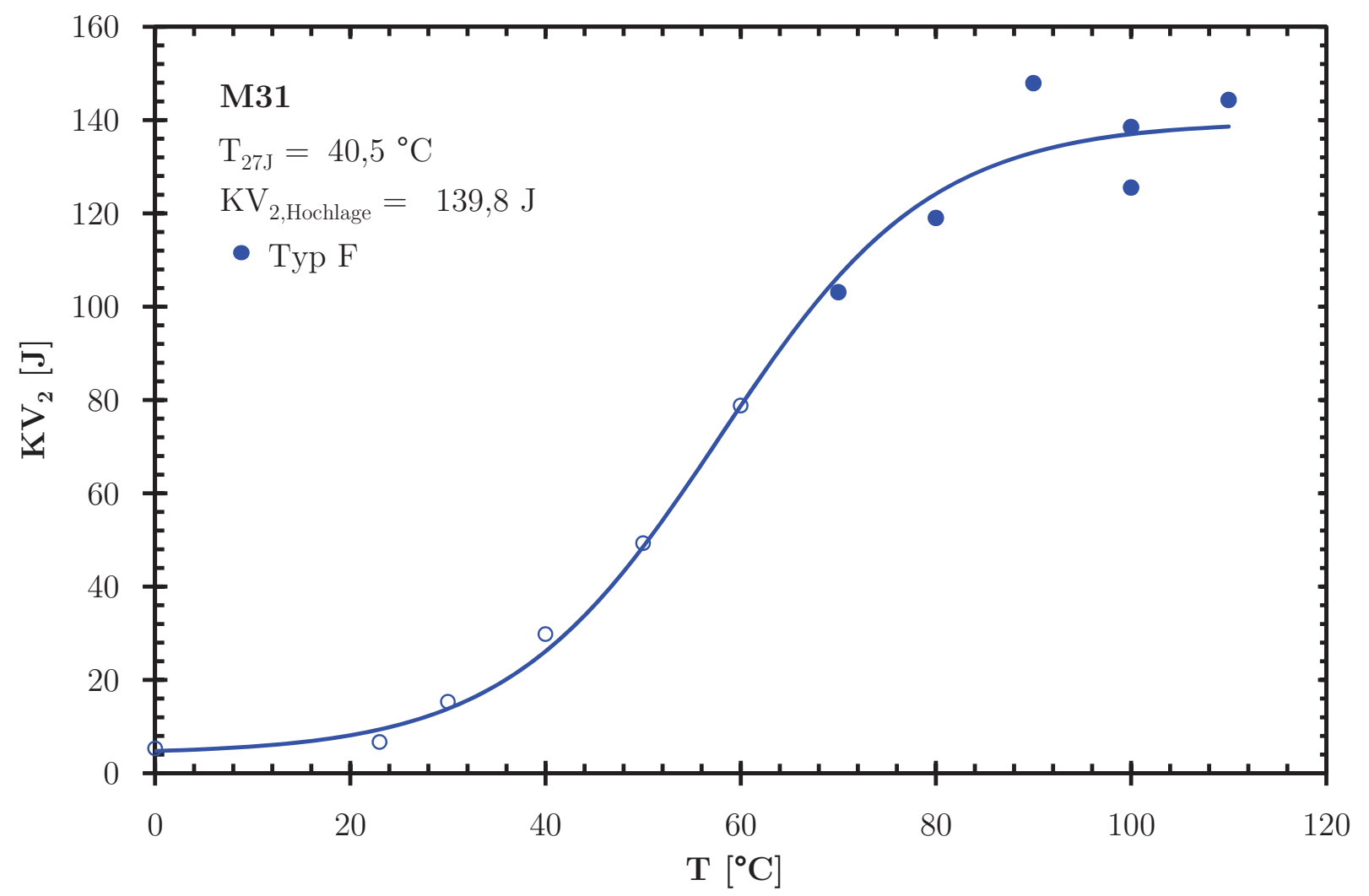

Abb. A.5.: Schlagarbeiten und Kerbschlag-Temperaturkurve der Probe M31

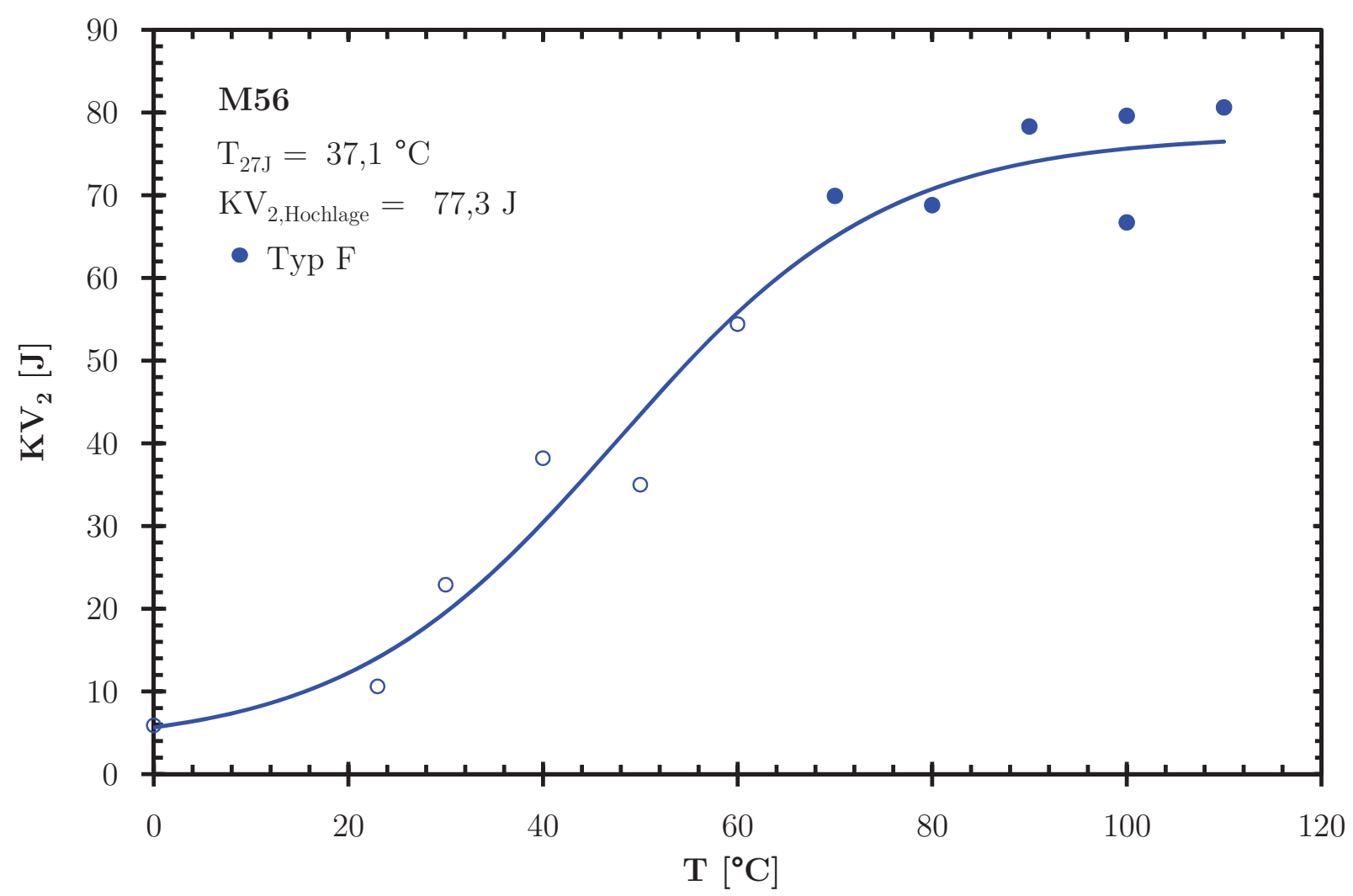

Abb. A.6.: Schlagarbeiten und Kerbschlag-Temperaturkurve der Probe M56 


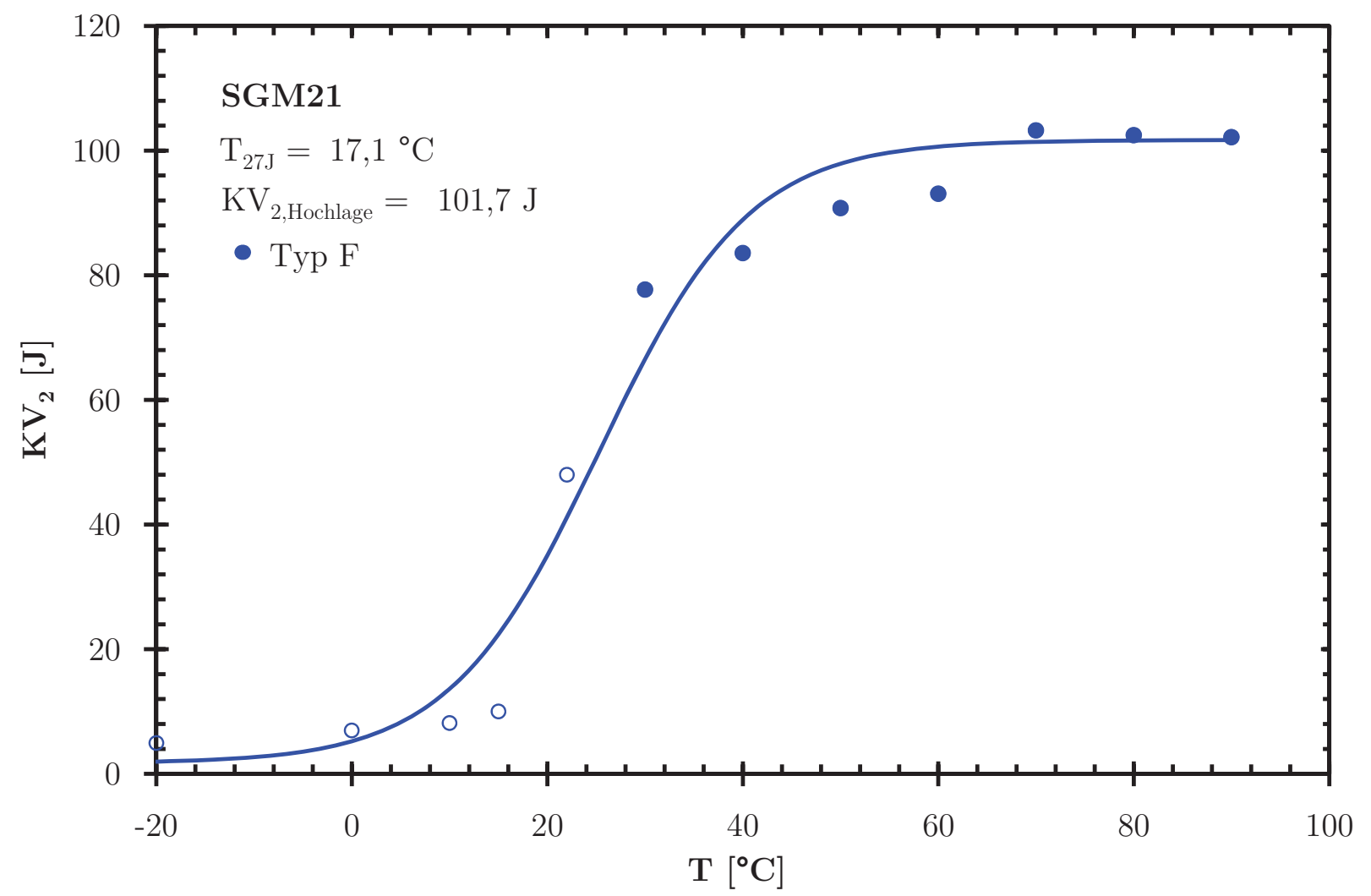

Abb. A.7.: Schlagarbeiten und Kerbschlag-Temperaturkurve der Probe SGM21

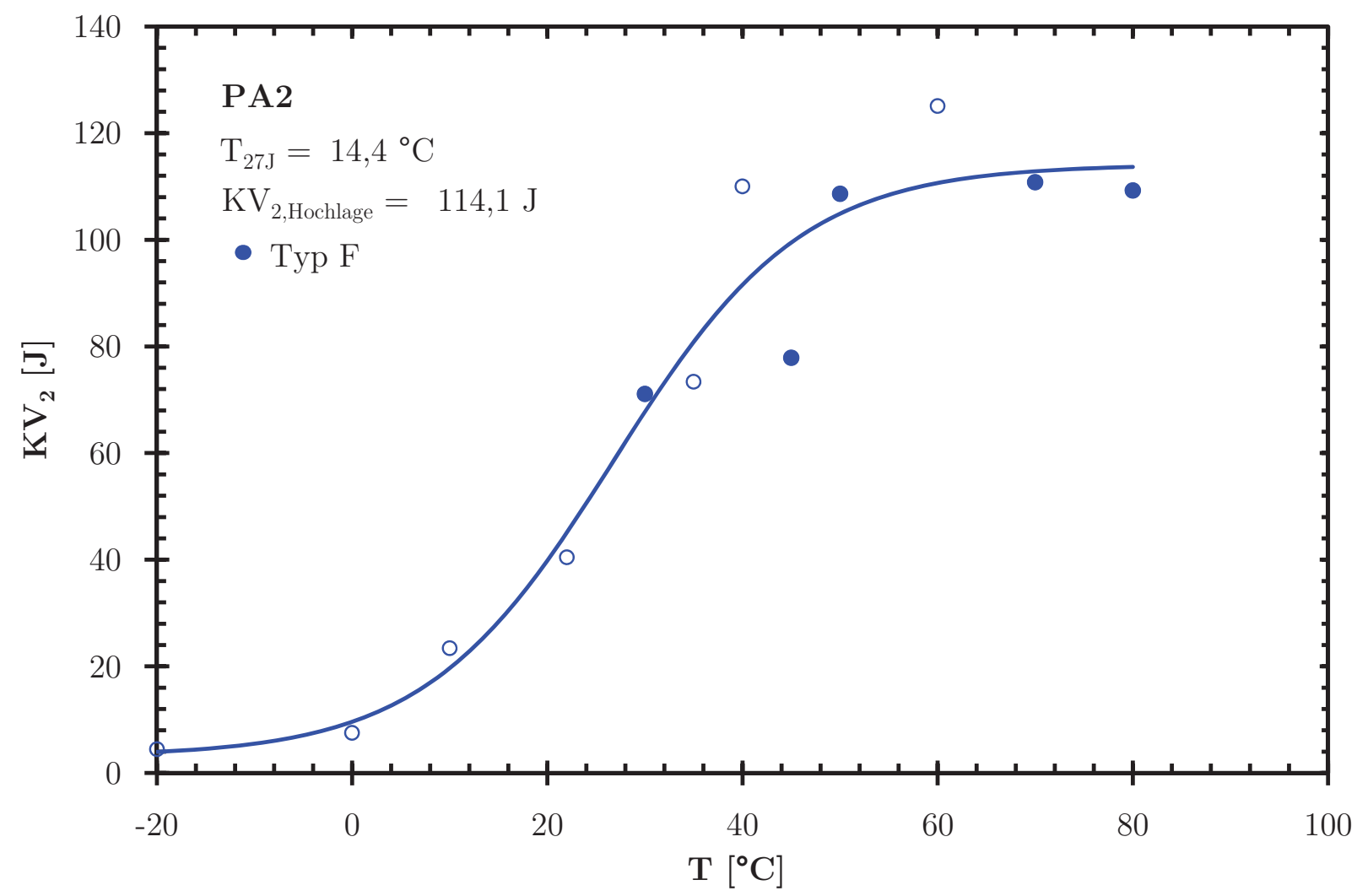

Abb. A.8.: Schlagarbeiten und Kerbschlag-Temperaturkurve der Probe PA2 


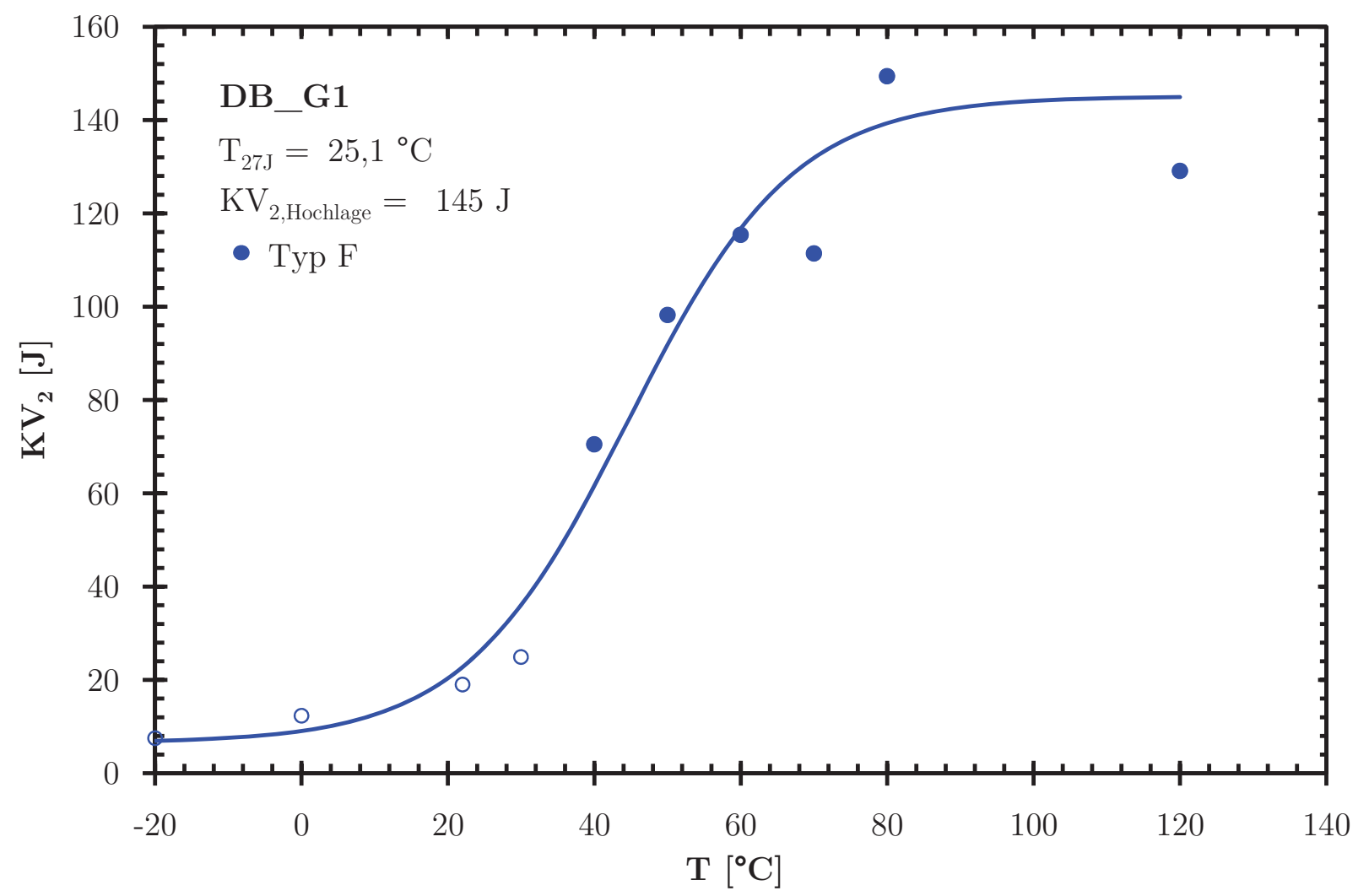

Abb. A.9.: Schlagarbeiten und Kerbschlag-Temperaturkurve der Probe DB_G1

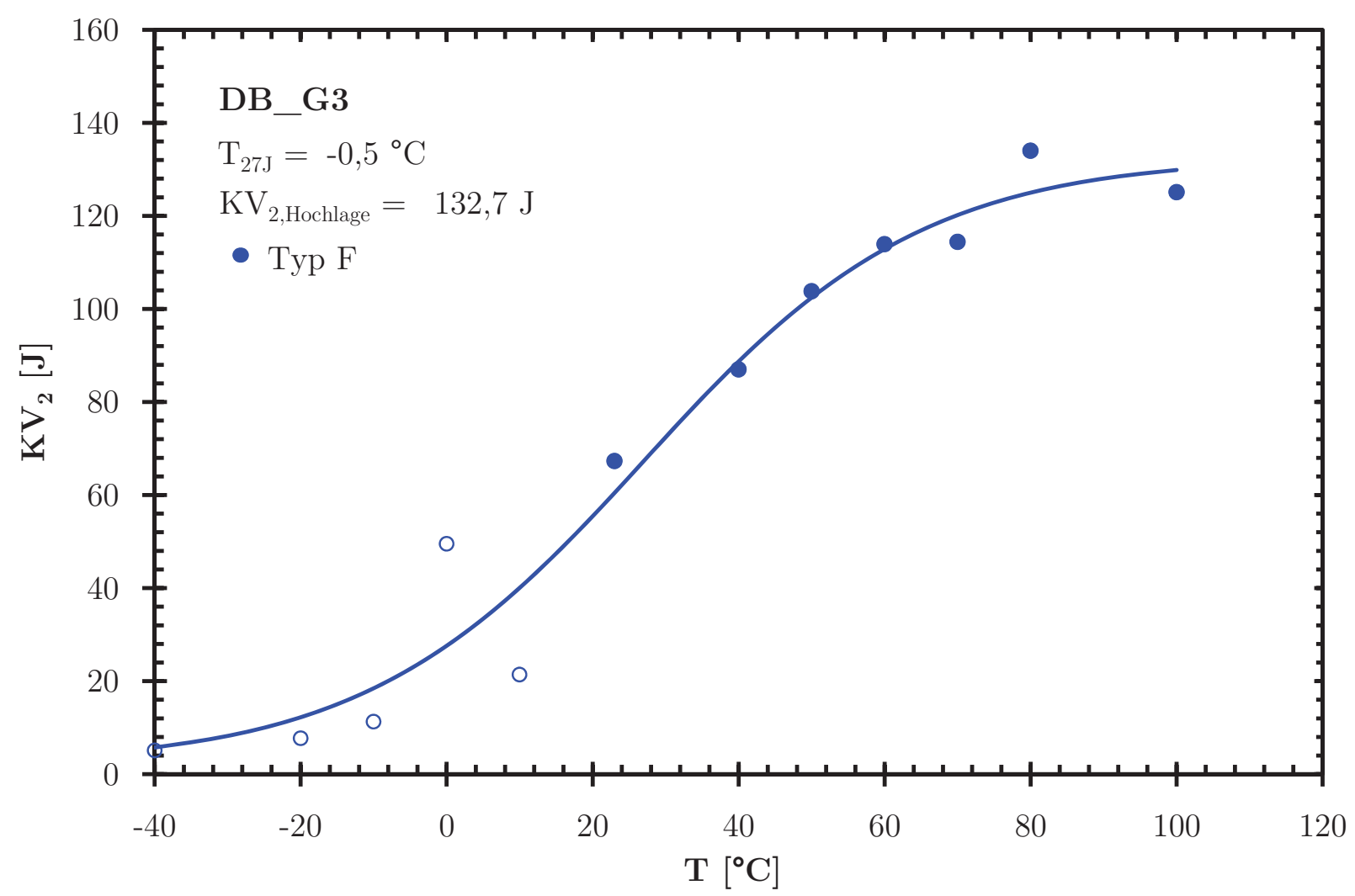

Abb. A.10.: Schlagarbeiten und Kerbschlag-Temperaturkurve der Probe DB_G3 


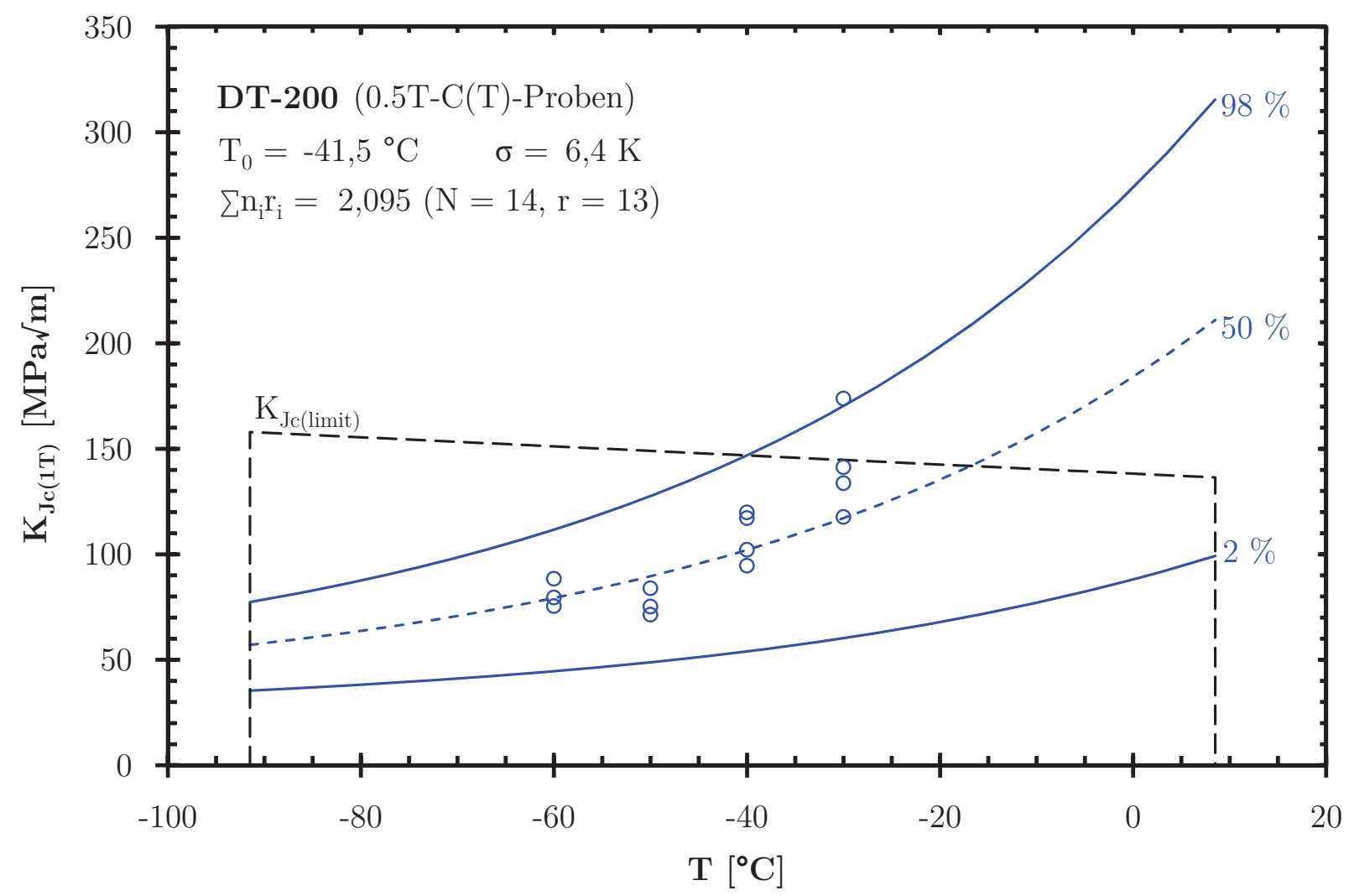

Abb. A.11.: Ergebnisse der Bruchmechanikversuche und Master-Curve-Analyse der Probe DT200

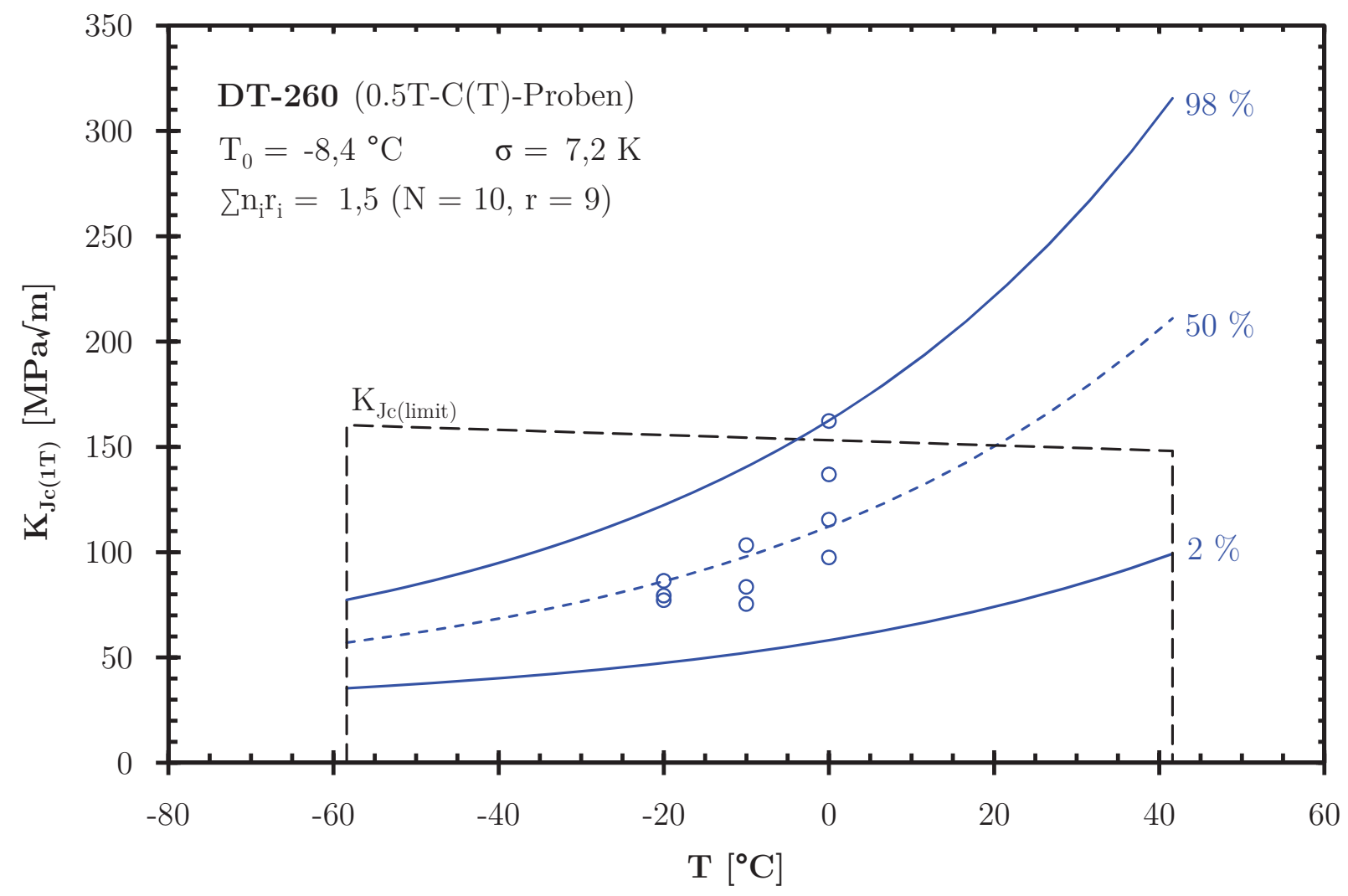

Abb. A.12.: Ergebnisse der Bruchmechanikversuche und Master-Curve-Analyse der Probe DT260 


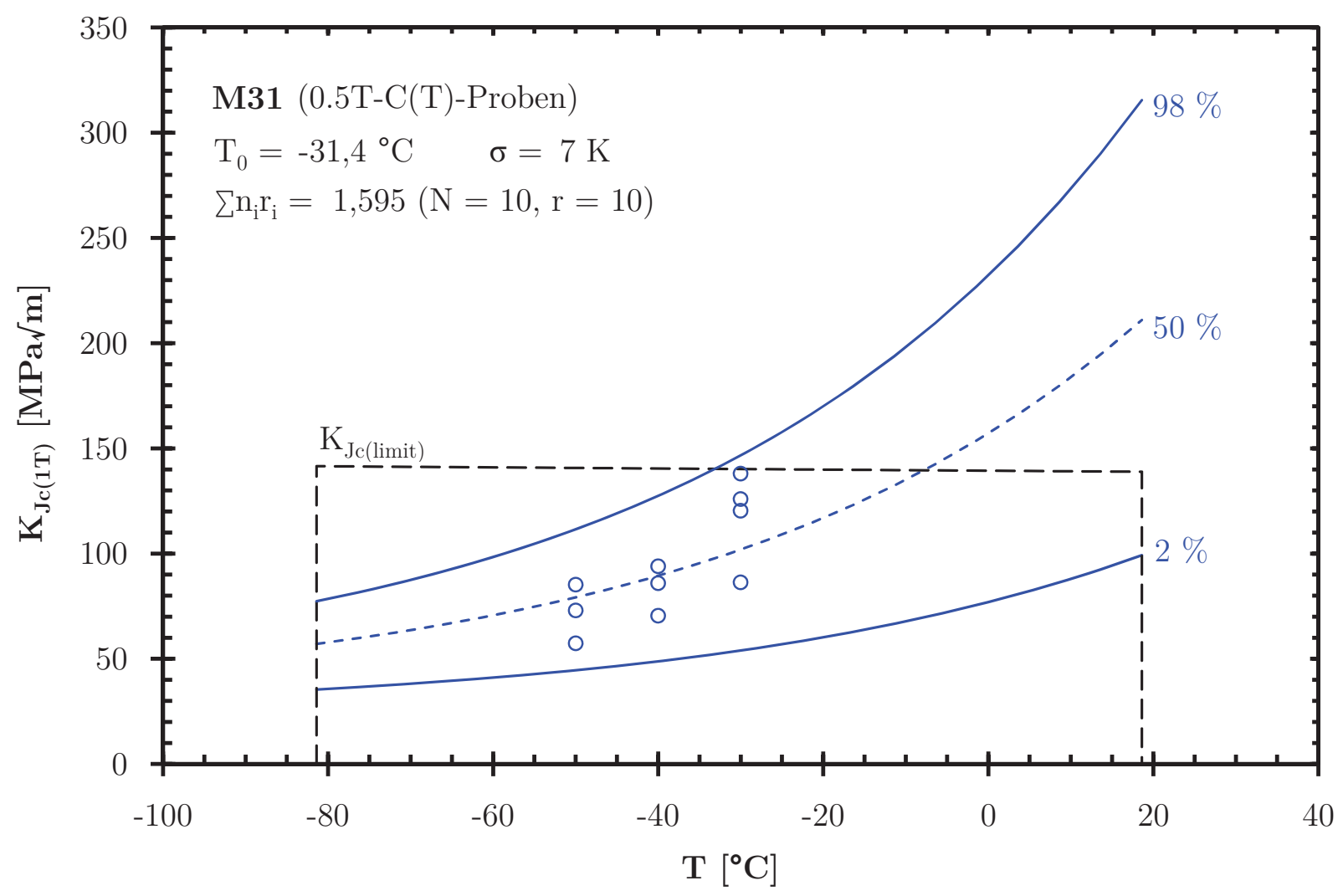

Abb. A.13.: Ergebnisse der Bruchmechanikversuche und Master-Curve-Analyse der Probe M31

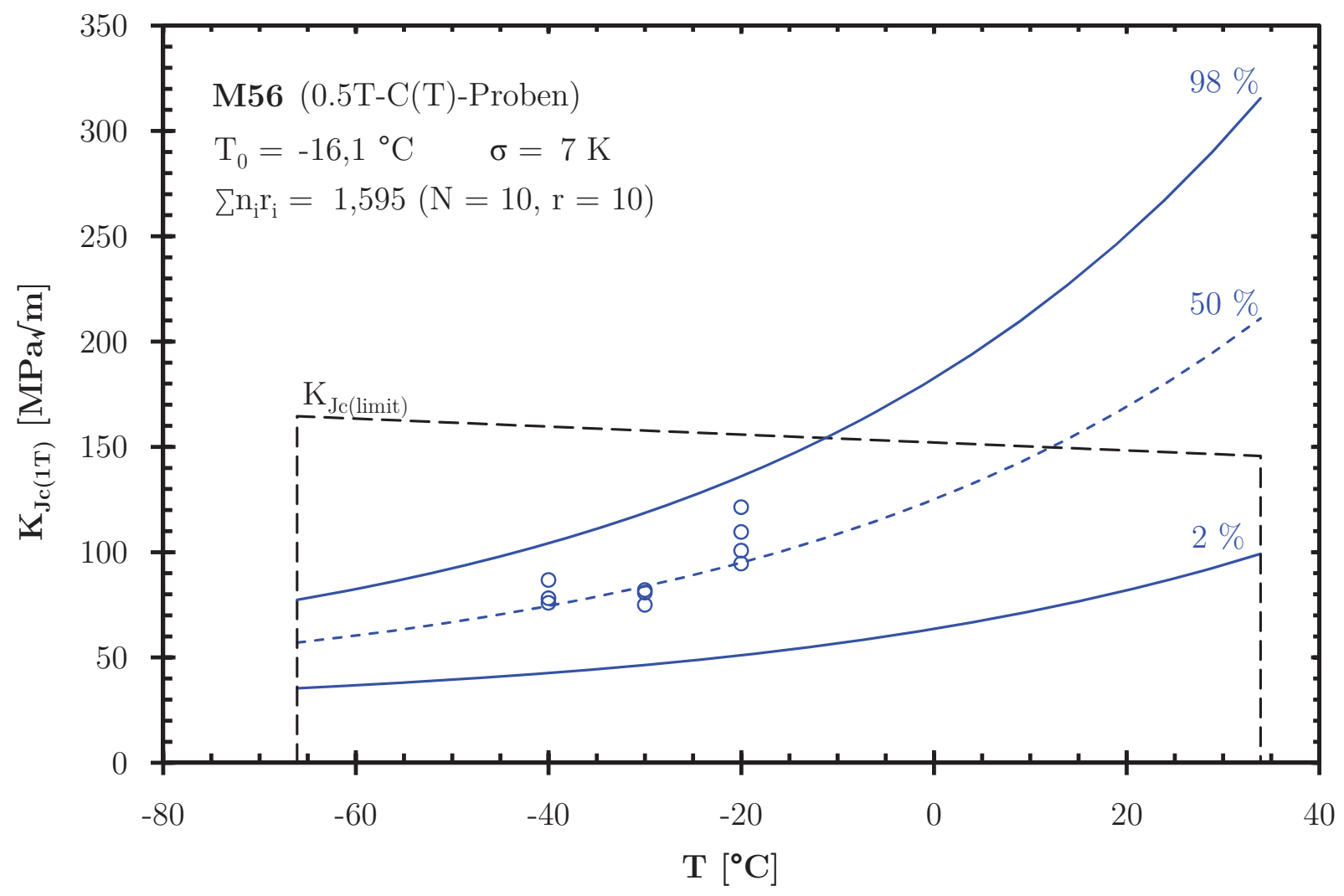

Abb. A.14.: Ergebnisse der Bruchmechanikversuche und Master-Curve-Analyse der Probe M56 


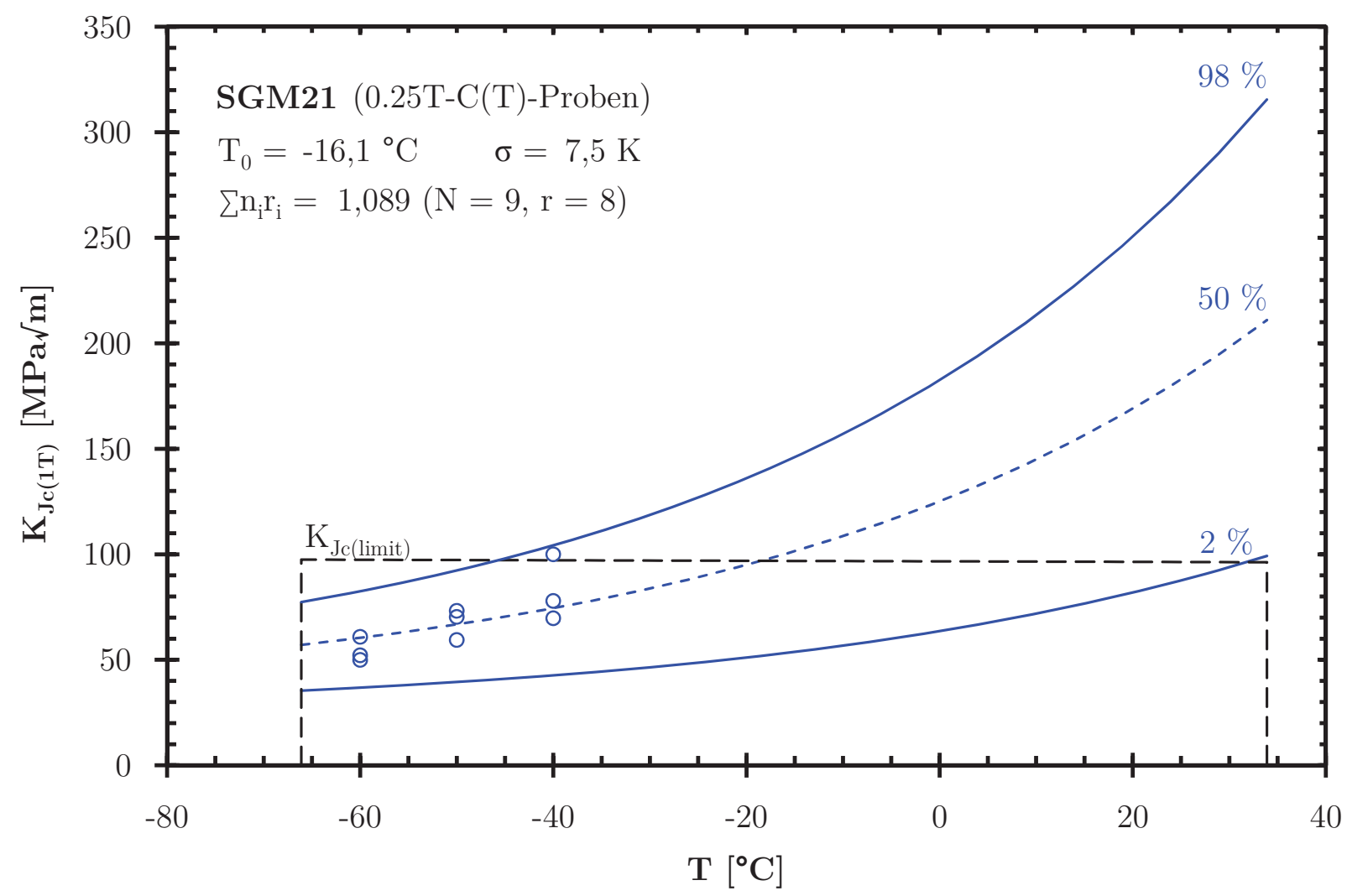

Abb. A.15.: Ergebnisse der Bruchmechanikversuche und Master-Curve-Analyse der Probe SGM21

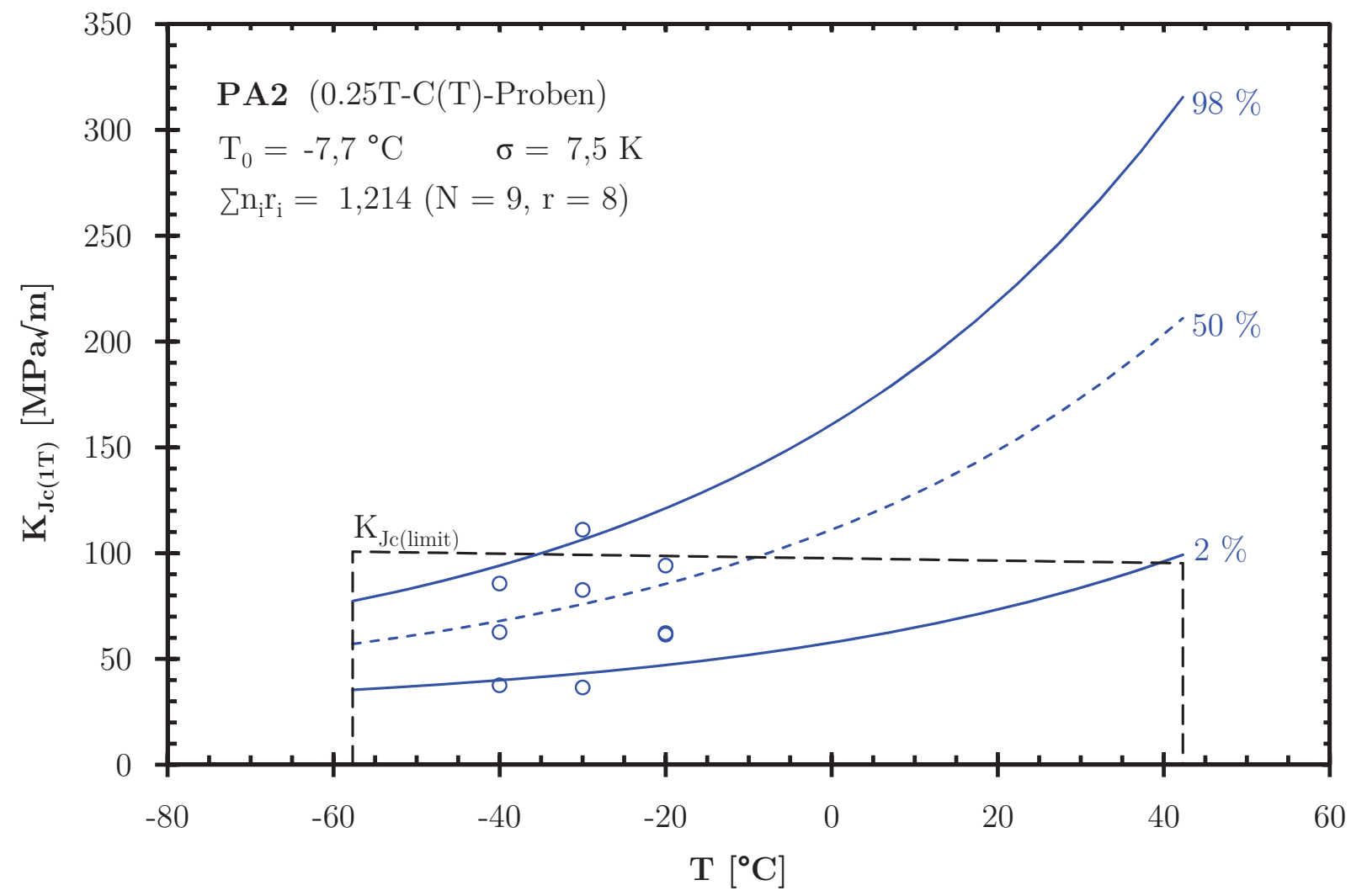

Abb. A.16.: Ergebnisse der Bruchmechanikversuche und Master-Curve-Analyse der Probe PA2 


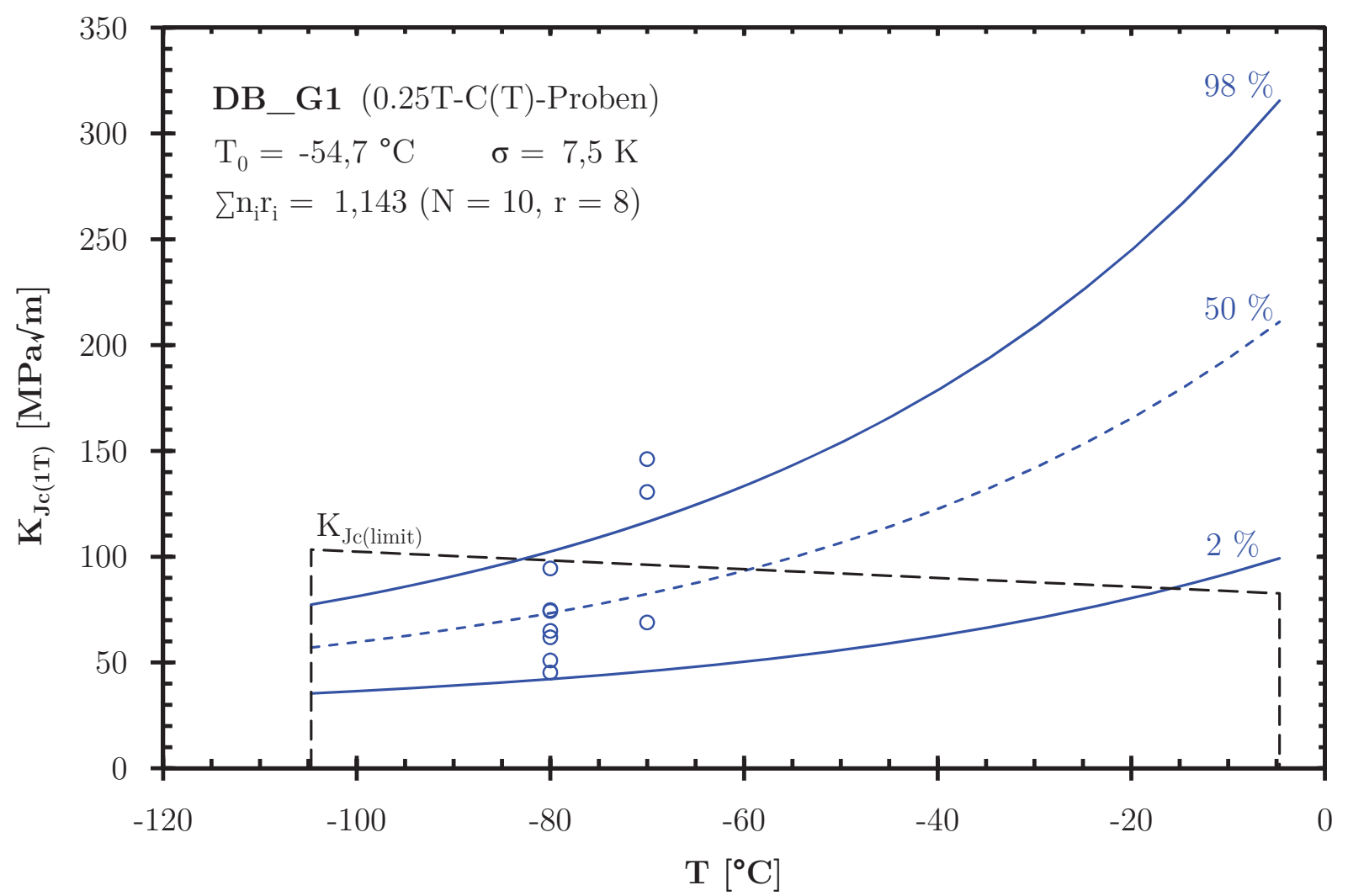

Abb. A.17.: Ergebnisse der Bruchmechanikversuche und Master-Curve-Analyse der Probe DB_G1

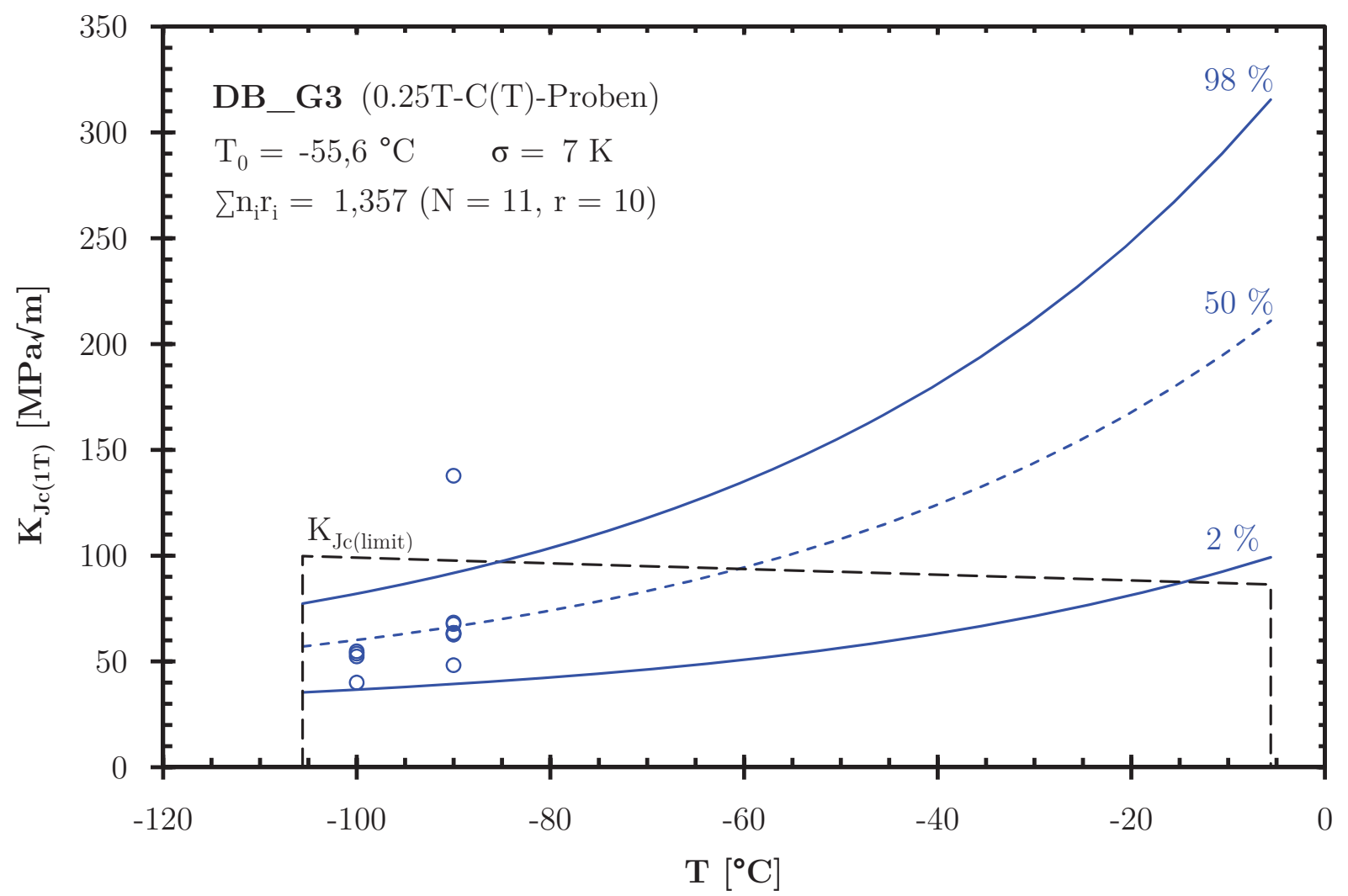

Abb. A.18.: Ergebnisse der Bruchmechanikversuche und Master-Curve-Analyse der Probe DB G3 


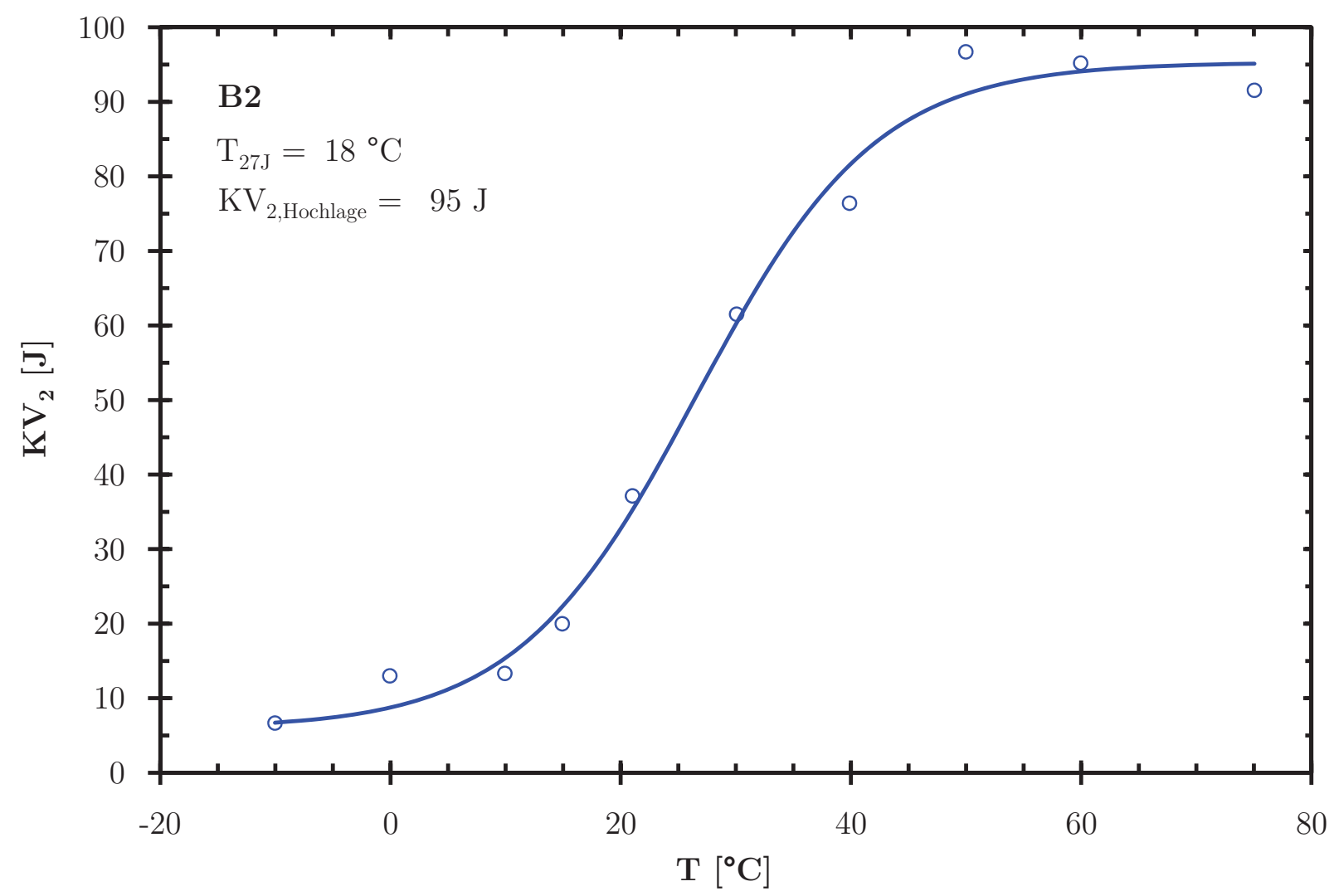

Abb. A.19.: Schlagarbeiten und Kerbschlag-Temperaturkurve der Probe B2

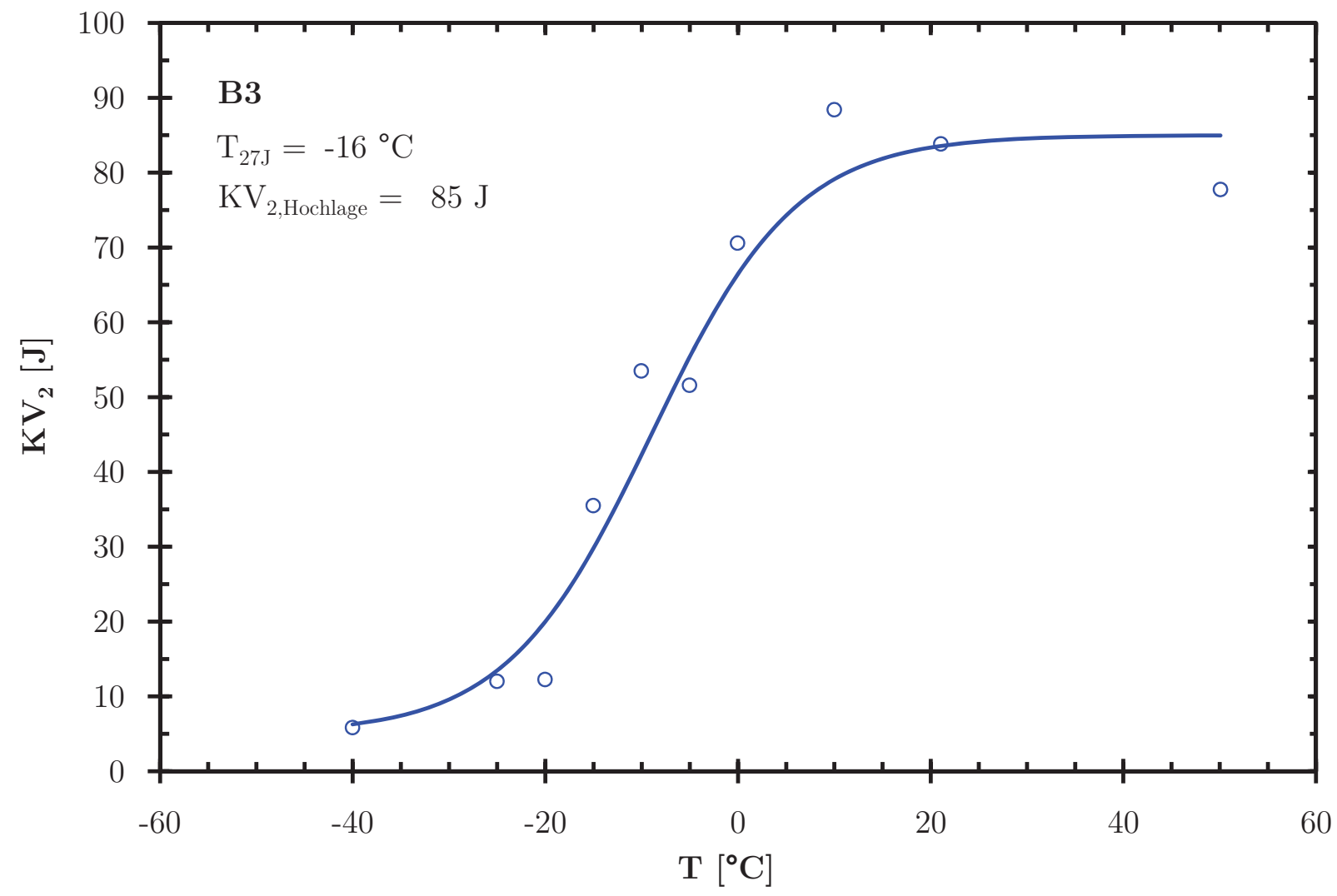

Abb. A.20.: Schlagarbeiten und Kerbschlag-Temperaturkurve der Probe B3 


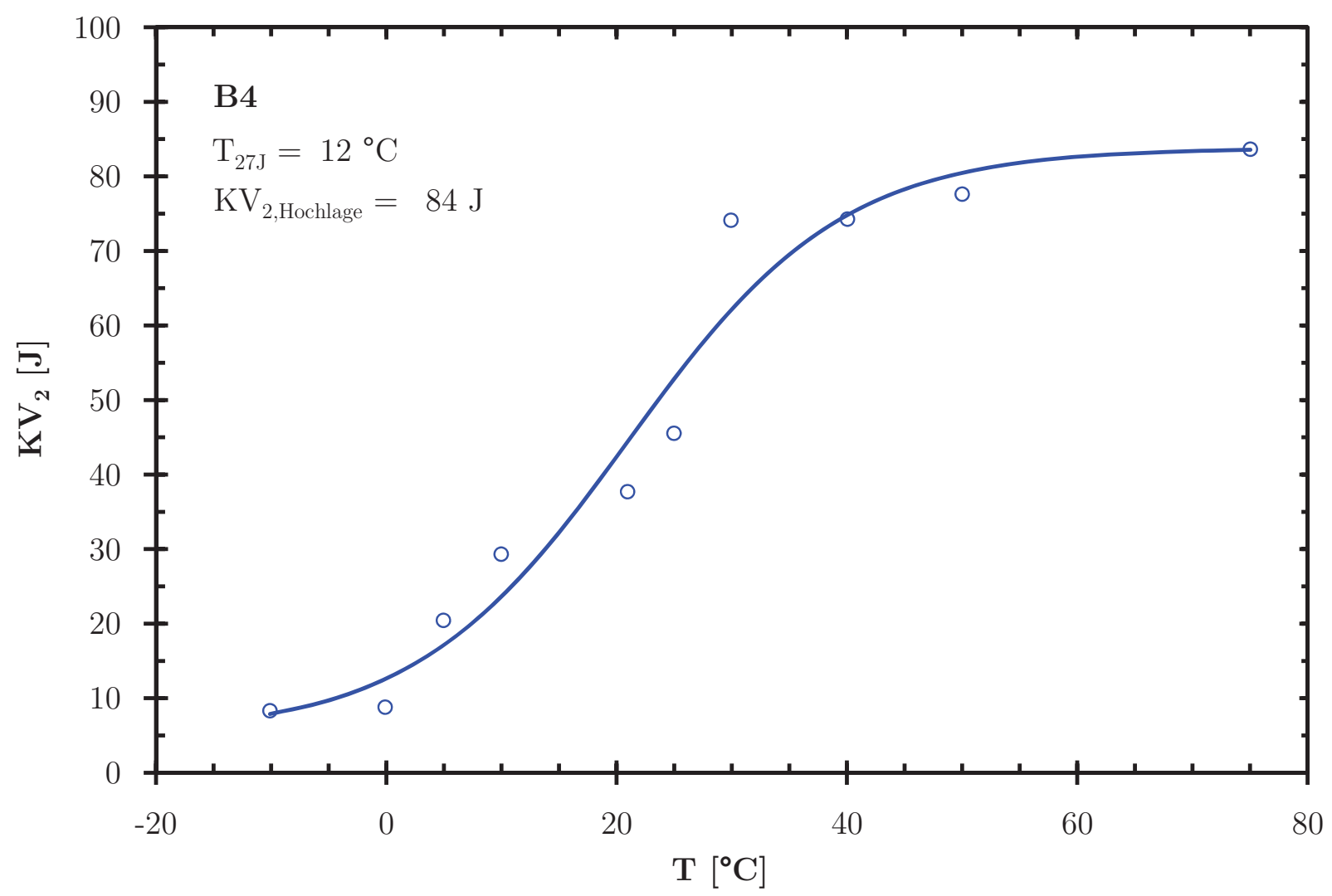

Abb. A.21.: Schlagarbeiten und Kerbschlag-Temperaturkurve der Probe B4

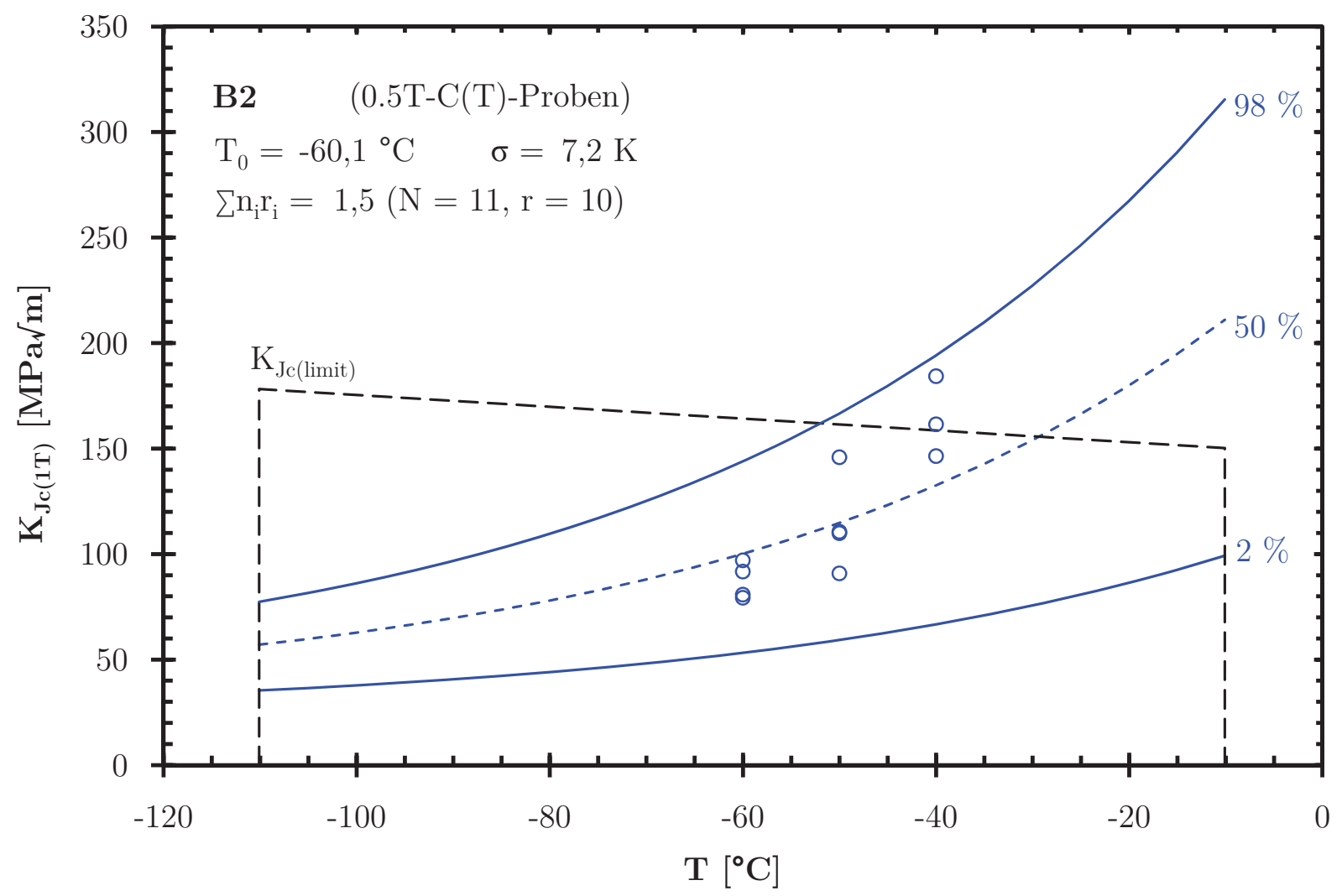

Abb. A.22.: Ergebnisse der Bruchmechanikversuche und Master-Curve-Analyse der Probe B2 


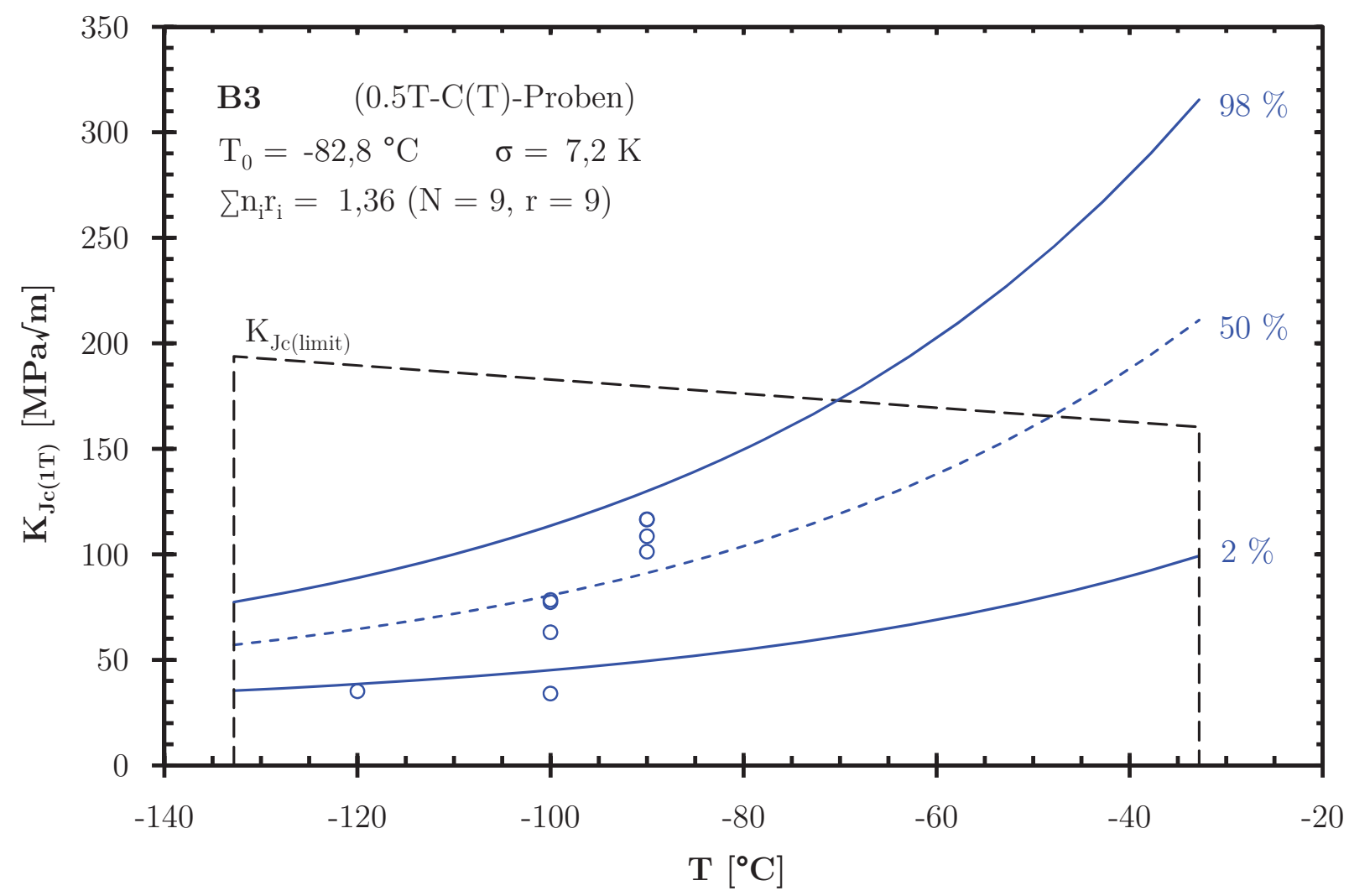

Abb. A.23.: Ergebnisse der Bruchmechanikversuche und Master-Curve-Analyse der Probe B3

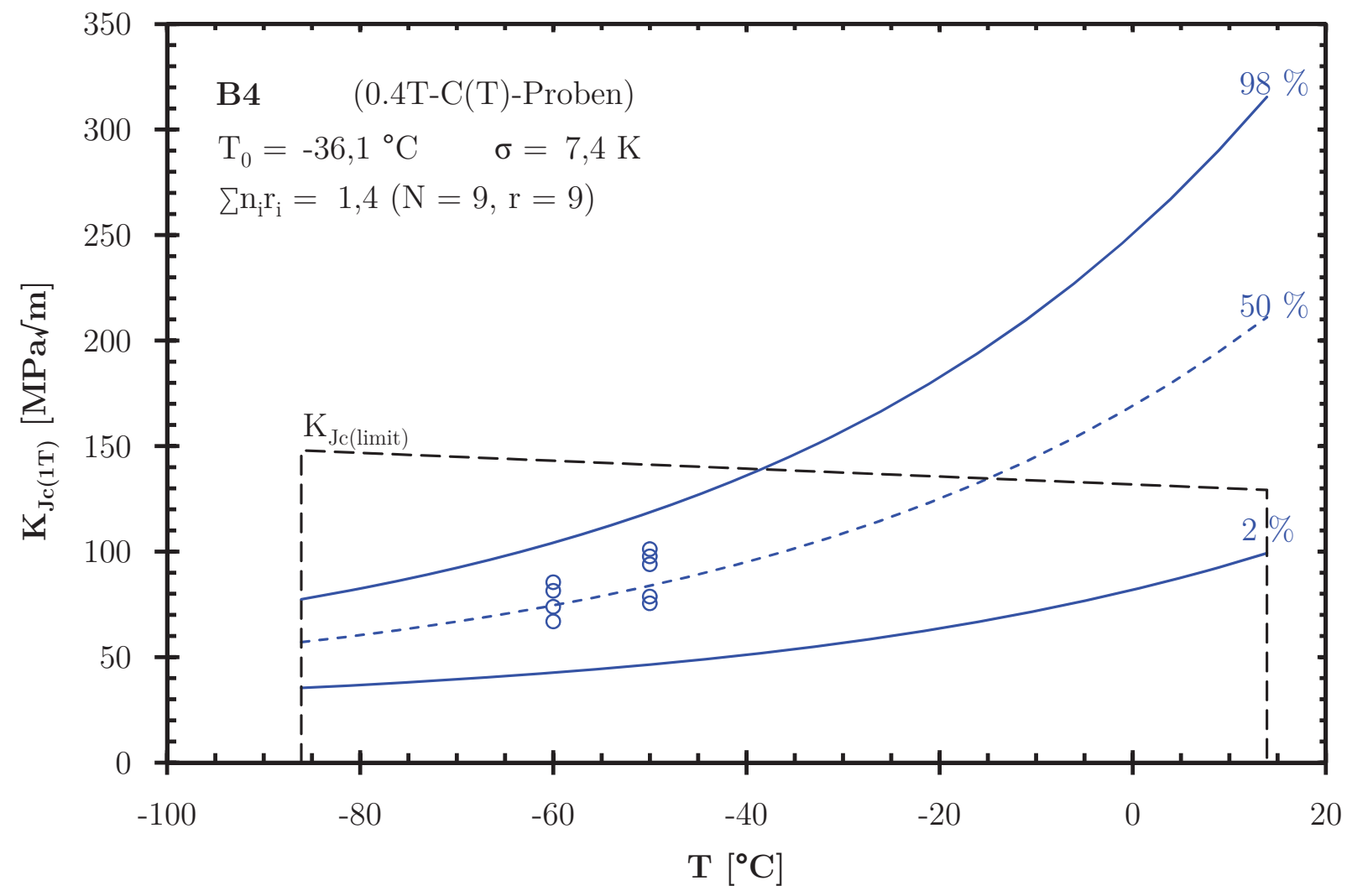

Abb. A.24.: Ergebnisse der Bruchmechanikversuche und Master-Curve-Analyse der Probe B4 

Anhang B.

Ergebnisse der Bauteilzugversuche 
Tab. B.1.: Ergebnisse der Bauteilzugversuche von Winkelprofilanschlüssen mit einem Verbindungsmittel

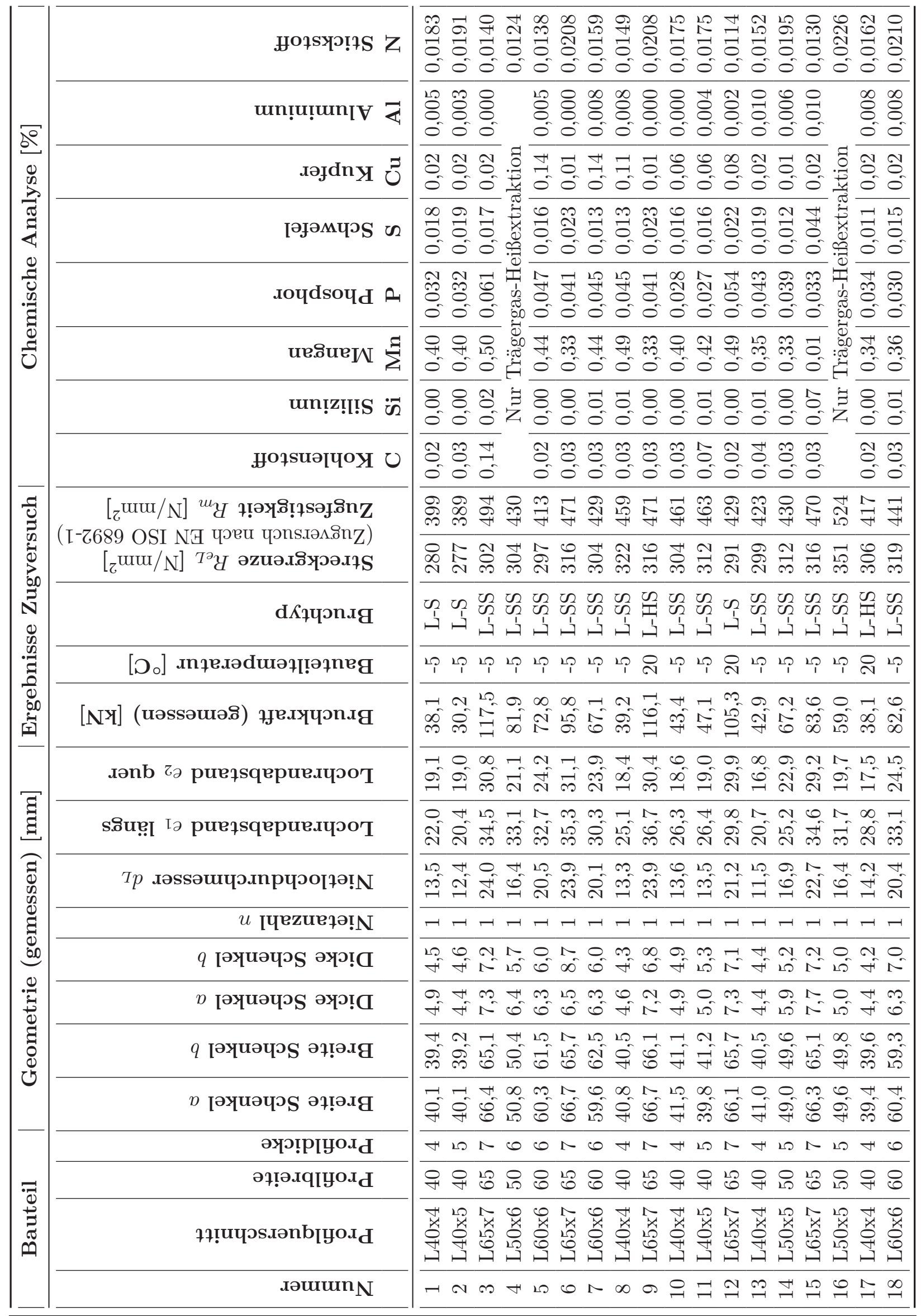


Tab. B.2.: Ergebnisse der Bauteilzugversuche von Winkelprofilanschlüssen mit mehreren Verbindungsmitteln

\begin{tabular}{|c|c|c|c|c|c|c|c|c|c|c|c|c|c|}
\hline \multicolumn{2}{|c|}{ Bauteil } & \multicolumn{6}{|c|}{ Geometrie (gemessen) [mm] } & \multicolumn{5}{|c|}{ Ergebnisse Zugversuch } & \multirow[t]{2}{*}{ Chemie } \\
\hline $\begin{array}{l}\dot{\bar{d}} \\
\stackrel{\Xi}{\Xi} \\
\dot{\Xi} \\
\text { Z }\end{array}$ & 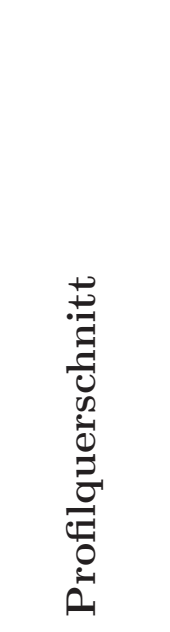 & 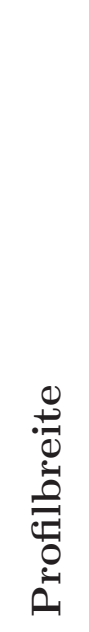 & 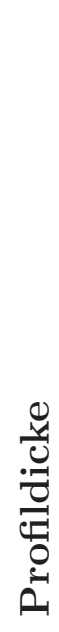 & 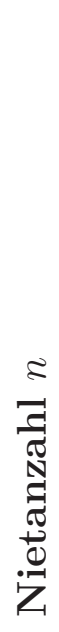 & 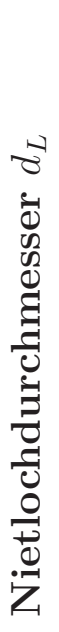 & 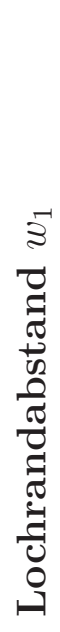 & 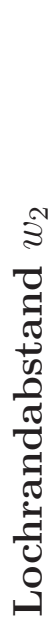 & 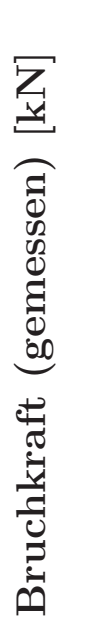 & 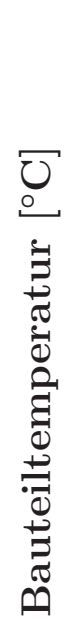 & 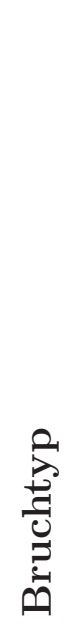 & 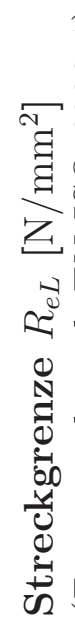 & 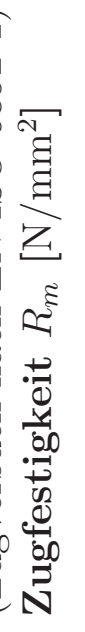 & \\
\hline 1 & L100x10 & 100 & 10 & 10 & 20 & 55 & & 480 & -20 & L-S & 291 & 390 & 0,0171 \\
\hline 2 & L130x12 & 130 & 12 & 10 & 24 & 50 & 90 & 960 & -20 & L-S & 311 & 409 & 0,0169 \\
\hline 3 & $\mathrm{~L} 65 \times 7$ & 65 & 7 & 8 & 16 & 35 & & 193 & -20 & $\mathrm{~L}-\mathrm{S}$ & 249 & 374 & 0,0132 \\
\hline 4 & L100x10 & 100 & 10 & 10 & 22 & 55 & & 479 & -20 & L-S & 242 & 358 & 0,0149 \\
\hline 5 & L130x12 & 130 & 12 & 10 & 26 & 65 & & 885 & -20 & L-S & 260 & 355 & 0,0128 \\
\hline
\end{tabular}

